\title{
Banach Algebras on Semigroups and on their Compactifications
}

\author{
H. G. Dales, A. T.-M. Lau, and D. Strauss
}

Author address:

Department of Pure Mathematics, University of Leeds, LEEDS LS2 9JT, United KINGDOM

E-mail address: garth@maths.leeds.ac.uk

Department of Mathematical and Statistical Sciences, University of Alberta, Edmonton, Alberta T6G 2G1, Canada

E-mail address: tlau@math.ualberta.ca

Department of Pure Mathematics, University of Leeds, LEEDS LS2 9JT, UNITED KingDOM

E-mail address: d.strauss@maths.leeds.ac.uk 



\section{Contents}

Chapter 1. Introduction 1

$\begin{array}{lll}\text { Chapter 2. Banach algebras and their second duals } & 15\end{array}$

$\begin{array}{lll}\text { Chapter 3. Semigroups } & 37\end{array}$

$\begin{array}{lll}\text { Chapter 4. Semigroup algebras } & 59\end{array}$

Chapter 5. Stone-Čech compactifications $\quad 75$

$\begin{array}{lll}\text { Chapter } 6 . & \text { The semigroup }(\beta S, \square) & 79\end{array}$

$\begin{array}{lll}\text { Chapter } 7 . & \text { Second duals of semigroup algebras } & 99\end{array}$

$\begin{array}{lll}\text { Chapter 8. Related spaces and compactifications } & 121\end{array}$

$\begin{array}{lll}\text { Chapter 9. Amenability for semigroups } & 133\end{array}$

$\begin{array}{lll}\text { Chapter 10. Amenability of semigroup algebras } & 147\end{array}$

Chapter 11. Amenability and weak amenability for certain $\begin{array}{ll}\text { Banach algebras } & 167\end{array}$

$\begin{array}{lll}\text { Chapter 12. Topological centres } & 175\end{array}$

$\begin{array}{lll}\text { Chapter 13. Open problems } & 199\end{array}$

$\begin{array}{ll}\text { Bibliography } & 201\end{array}$

$\begin{array}{ll}\text { Index } & 209\end{array}$

Index of Symbols 213 


\begin{abstract}
Let $S$ be a (discrete) semigroup, and let $\ell^{1}(S)$ be the Banach algebra which is the semigroup algebra of $S$. We shall study the structure of this Banach algebra and of its second dual.

We shall determine exactly when $\ell^{1}(S)$ is amenable as a Banach algebra, and shall discuss its amenability constant, showing that there are 'forbidden values' for this constant.

The second dual of $\ell^{1}(S)$ is the Banach algebra $M(\beta S)$ of measures on the Stone-Cech compactification $\beta S$ of $S$, where $M(\beta S)$ and $\beta S$ are taken with the first Arens product $\square$. We shall show that $S$ is finite whenever $M(\beta S)$ is amenable, and we shall discuss when $M(\beta S)$ is weakly amenable. We shall show that the second dual of $L^{1}(G)$, for $G$ a locally compact group, is weakly amenable if and only if $G$ is finite.

We shall also discuss left-invariant means on $S$ as elements of the space $M(\beta S)$, and determine their supports.

We shall show that, for each weakly cancellative and nearly right cancellative semigroup $S$, the topological centre of $M(\beta S)$ is just $\ell^{1}(S)$, and so $\ell^{1}(S)$ is strongly Arens irregular; indeed, we shall considerably strengthen this result by showing that, for such semigroups $S$, there are two-element subsets of $\beta S \backslash S$ that are determining for the topological centre; for more general semigroups $S$, there are finite subsets of $\beta S \backslash S$ with this property.

We have partial results on the radical of the algebras $\ell^{1}(\beta S)$ and $M(\beta S)$.

We shall also discuss analogous results for related spaces such as $W A P(S)$ and $L U C(G)$.

2000 Mathematics Subject Classification. Primary 43A10, 43A20; secondary 46J10.

Key words and phrases. Banach algebras, second duals, dual Banach algebras, Arens products, Arens regular, topological centre, strongly Arens irregular, introverted subspace, amenable, weakly amenable, approximately amenable, amenability constant, ultrafilter Stone-Cech compactification, invariant mean, semigroup, Rees semigroup, semigroup algebra, Munn algebra, semi-character, character, cancellative semigroup, radical, minimal ideal, idempotent, locally compact group, group algebra, measure algebra, bounded approximate identity, diagonal.
\end{abstract}




\section{CHAPTER 1}

\section{Introduction}

Our aim in this memoir is to study the algebraic structure of some Banach algebras which are defined on semigroups and on their compactifications. In particular we shall study the semigroup algebra $\ell^{1}(S)$ of a semigroup $S$ and its second dual algebra; this includes the important special case in which $S$ is a group. Here $\ell^{1}(S)$ is taken with the convolution product $\star$ and the second dual $\ell^{1}(S)^{\prime \prime}$ is taken with respect to the first and second Arens products, $\square$ and $\diamond$; these second dual algebras are identified with Banach algebras $(M(\beta S), \square)$ and $(M(\beta S), \diamond)$, which are, respectively, the right and left topological semigroups of measures defined on $\beta S$, the Stone-Cech compactification of $S$. We shall also study the closed subalgebras $\ell^{1}(\beta S)$ of $M(\beta S)$ and some related Banach algebras.

Much of our work depends on knowledge of the properties of the semigroup $(\beta S, \square)$, and, in particular, of $(\beta \mathbb{N}, \square)$. We wish to stress that $(\beta \mathbb{N}, \square)$ is a deep, subtle, and significant mathematical object, with a distinguished history and about which there are challenging open questions; we hope to introduce the power of this semigroup to those primarily interested in Banach algebras. Indeed the questions that we ask about Banach algebras are often resolved by inspecting the properties of this semigroup, and sometimes require new results about it. So we also hope that those primarily interested in topological semigroups will be stimulated by the somewhat broader questions, arising from Banach algebra theory, that we raise about $(\beta S, \square)$. In brief, we aspire to interest specialists in both Banach algebra theory and in topological semigroups in our work. For this reason we have tried to incorporate general background from each of these theories in our exposition in an attempt to make the work accessible to both communities.

This paper is partially a sequel to the earlier memoir [21]. (For a correction to [21], see p. 198 of the present work.)

Notation We recall some notation that will be used throughout; for further details of all terms used, see [19] and [21].

We shall use elementary properties of ordinal 
and cardinal

numbers as given in [19, Chapter 1.1], for example. The minimum infinite ordinal is $\omega$ and the minimum uncountable ordinal is $\omega_{1}$; these ordinals are also cardinals, and are denoted by $\aleph_{0}$ and $\aleph_{1}$, respectively, in this case. The cofinality of an ordinal $\alpha$ is $\operatorname{cof} \alpha$; a cardinal $\alpha$ is regular

if $\operatorname{cof} \alpha=\alpha$.

The Continuum Hypothesis $(\mathrm{CH})$

is the assertion that the continuum $\mathfrak{c}=2^{\aleph_{0}}$ is equal to $\aleph_{1}$; this hypothesis is independent of the usual axioms ZFC of set theory. Results that are claimed only in the theory $\mathrm{ZFC}+\mathrm{CH}$ are denoted by ' $(\mathrm{CH})$ '.

Let $S$ be a set. The cardinality $S$ is denoted by $|S|$, the family of all subsets of $S$ is $\mathcal{P}(S)$, and the family of all finite subsets of $S$ is $\mathcal{P}_{f}(S)$. Let $\kappa$ be a cardinal. Then

$$
[S]^{\kappa}=\{T \subset S:|T|=\kappa\} \quad \text { and } \quad[S]^{<\kappa}=\{T \subset S:|T|<\kappa\} .
$$

The characteristic function of a subset $T$ of $S$ is denoted by $\chi_{T}$; we set $\delta_{s}=\chi_{\{s\}}$ for $s \in S$.

We set $\mathbb{N}=\{1,2, \ldots\}, \mathbb{Z}=\{0, \pm 1, \pm 2, \ldots\}$, and $\mathbb{Z}^{+}=\{0,1,2, \ldots\}$. The sets $\{1, \ldots, n\}$ and $\{0,1, \ldots, n\}$ are denoted by $\mathbb{N}_{n}$ and $\mathbb{Z}_{n}^{+}$, respectively. The set of rational numbers is $\mathbb{Q}, \mathbb{I}=[0,1]$, and the unit circle and open unit disc in the complex plane $\mathbb{C}$ are denoted by $\mathbb{T}$ and $\mathbb{D}$, respectively. The complex conjugate of $z \in \mathbb{C}$ is denoted by $\bar{z}$.

Algebras Let $A$ be an algebra

(always over the complex field, $\mathbb{C}$ ). The product map is

$$
m_{A}:(a, b) \mapsto a b, \quad A \times A \rightarrow A .
$$

The opposite algebra

to $A$ is denoted by $A^{\text {op }}$; this algebra has the product $(a, b) \mapsto$ ba, $A \times A \rightarrow A$. In the case where $A$ does not have an identity, the algebra formed by adjoining an identity to $A$ is $A^{\#}$ (and $A^{\#}=A$ if $A$ has an identity); the identity of $A$ or $A^{\#}$ is often denoted by $e_{A}$.

The centre

of $A$ is

$$
\mathfrak{Z}(A)=\{a \in A: a b=b a(b \in A)\} .
$$

An idempotent

in $A$ is an element $p$ such that $p^{2}=p$; the family of idempotents in $A$ is denoted by $\mathfrak{I}(A)$. For $p, q \in \mathfrak{I}(A)$, set $p \leq q$ if $p q=q p=p$, so that $(\mathfrak{I}(A), \leq)$ is a partially ordered set; a minimal idempotent in $A$ is a minimal element of the set $(\mathfrak{I}(A) \backslash\{0\}, \leq)$. 
Let $I$ be an ideal in an algebra $A$, and let $B$ be a subalgebra of $A$ such that $A=B \oplus I$ as a linear space. Then $A$ is the semi-direct product

of $B$ and $I$, written $A=B \ltimes I$.

Let $I$ be an ideal in an algebra $A$, and suppose that $I$ has an identity $e_{I}$. Then we remark that $e_{I} \in \mathfrak{Z}(A)$. Indeed, for each $a \in A$ we have $a e_{I}, e_{I} a \in I$, and so $e_{I}\left(a e_{I}\right)=a e_{I}$ and $\left(e_{I} a\right) e_{I}=e_{I} a$. Thus $e_{I} \in \mathfrak{Z}(A)$.

Let $A$ be an algebra. We denote by $R_{A}, N_{A}$, and $Q_{A}$ the (Jacobson) radical

of $A$, the set of nilpotent

elements of $A$, and the set of quasi-nilpotent

elements of $A$, respectively; the sets $R_{A}, N_{A}$, and $Q_{A}$ are defined in [19], but $R_{A}$ is denoted by $\operatorname{rad} A$ in [19]. We recall that $R_{A}$ is defined to be the intersection of the maximal modular left ideals of $A$, that $R_{A}$ is an ideal in $A$, and that $A$ is defined to be semisimple

if $R_{A}=\{0\}$. We always have the trivial inclusions:

$$
R_{A} \subset Q_{A}, \quad N_{A} \subset Q_{A} .
$$

In general, we have $R_{A} \not \subset N_{A}$ and $N_{A} \not \subset R_{A}$; further, neither $Q_{A}$ nor $N_{A}$ is necessarily closed under either addition or multiplication in $A$. For an ideal $I$ in $A$, we have $R_{I}=I \cap R_{A}$; in the case where $A / I$ is semisimple, we have $R_{I}=R_{A}$.

A nilpotent element $a \in A$ has index $n$ if $n=\min \left\{k \in \mathbb{N}: a^{k}=0\right\}$; a subset $S$ of $A$ is nil

if each element of $S$ is nilpotent; the radical $R_{A}$ contains each left or right ideal which is nil.

Let $A$ be an algebra. For subsets $S$ and $T$ of $A$, we set

$$
S \cdot T=\{s t: s \in S, t \in T\},
$$

and $S T=\operatorname{lin} S \cdot T$; we write $S^{[2]}$ for $S \cdot S$; we define $S^{n}$ inductively by setting $S^{n+1}=S S^{n}(n \in \mathbb{N})$. The set $S$ is nilpotent

if $S^{n}=\{0\}$ for some $n \in \mathbb{N}$. The algebra $A$ factors

if $A=A^{[2]}$.

Let $A$ be an algebra, and let $a \in A$. Then $a$ is quasi-invertible

if there exists $b \in A$ such that

$$
a+b-a b=a+b-b a=0
$$

the set of quasi-invertible elements of $A$ is denoted by $q$ - $\operatorname{Inv} A$. A subalgebra $B$ of $A$ is full

if $B \cap q-\operatorname{Inv} A=q-\operatorname{Inv} B$. In the case where $A$ has an identity, the set of invertible elements of $A$ is denoted by $\operatorname{Inv} A$, and the spectrum 
of an element $a \in A$ is denoted by $\sigma_{A}(a)$ or $\sigma(a)$, so that

$$
\sigma_{A}(a)=\left\{z \in \mathbb{C}: z e_{A}-a \notin \operatorname{Inv} A\right\} ;
$$

if $B$ is a full subalgebra of $A$ and $a \in B$, then $\sigma_{B}(a)=\sigma_{A}(a)$.

A character

on an algebra $A$ is a non-zero homomorphism from $A$ onto $\mathbb{C}$; the collection of characters on $A$ is the character space of $A$ and is denoted by $\Phi_{A}$. Suppose that $\Phi_{A} \neq \emptyset$, and take $a \in A$. Then the Gel'fand transform

of $a$ is defined to be $\widehat{a} \in \mathbb{C}^{\Phi_{A}}$, where $\widehat{a}(\varphi)=\varphi(a) \quad\left(\varphi \in \Phi_{A}\right)$; we define the Gel'fand transform of $A$ as

$$
\mathcal{G}: a \mapsto \widehat{a}, \quad A \rightarrow \mathbb{C}^{\Phi_{A}},
$$

so that $\mathcal{G}$ is a homomorphism.

Let $m, n \in \mathbb{N}$, and let $S$ be a set. The collection of $m \times n$ matrices with entries from $S$ is denoted by $\mathbb{M}_{m, n}(S)$, with $\mathbb{M}_{n}(S)$ for $\mathbb{M}_{n, n}(S)$ and $\mathbb{M}_{m, n}$ for $\mathbb{M}_{m, n}(\mathbb{C})$. In particular, $\mathbb{M}_{n}$ is a unital algebra; the matrix units

in $\mathbb{M}_{n}$ are denoted by $E_{i j}$, so that

$$
E_{i j} E_{k \ell}=\delta_{j, k} E_{i \ell} \quad\left(i, j, k, \ell \in \mathbb{N}_{n}\right),
$$

and the identity matrix in $\mathbb{M}_{n}$ is $I_{n}=\left(\delta_{i, j}\right)$. Let $A$ be an algebra. Then $\mathbb{M}_{n}(A)$ is also an algebra in the obvious way; the matrix $\left(a_{i j}\right)$ is identified with $\sum\left\{E_{i j} \otimes a_{i j}: i, j \in \mathbb{N}_{n}\right\}$, so that $\mathbb{M}_{n}(A)$ is isomorphic to $\mathbb{M}_{n} \otimes A$. If $A$ is unital, we regard $\mathbb{M}_{n}$ as a subset of $\mathbb{M}_{n}(A)$ by identifying $E_{i j}$ with $E_{i j} \otimes e_{A}$. In the case where $A$ is commutative, the determinant, $\operatorname{det} a$,

of an element $a \in \mathbb{M}_{n}(A)$ is defined in the usual way; the element $a$ is invertible in $\mathbb{M}_{n}(A)$ if and only if det $a$ is invertible in $A$.

Let $E$ be a linear space. The linear span of a subset $S$ of $E$ is denoted by $\operatorname{lin} S$. Let $S$ and $T$ be subsets of $E$. Then

$$
S+T=\{s+t: s \in S, t \in T\} .
$$

The linear space of all linear operators from $E$ to a linear space $F$ is denoted by $\mathcal{L}(E, F)$, and we write $\mathcal{L}(E)$ for the space $\mathcal{L}(E, E)$. In fact, $\mathcal{L}(E)$ is an algebra with respect to composition of operators, with identity $I_{E}$, the identity operator on $E$.

Let $A$ be an algebra, and let $E$ be an $A$-bimodule with respect to operations $(a, x) \mapsto a \cdot x$ and $(a, x) \mapsto x \cdot a$ from $A \times E$ to $E$. For subsets $S$ of $A$ and $T$ of $E$, set

$$
S \cdot T=\{a \cdot x: a \in S, x \in T\},
$$

and $S T=\operatorname{lin} S \cdot T$. A map $D \in \mathcal{L}(A, E)$ is a derivation 
if

$$
D(a b)=D(a) \cdot b+a \cdot D(b) \quad(a, b \in A) .
$$

For $x \in E$, set

$$
a d_{x}: a \mapsto a \cdot x-x \cdot a, \quad A \rightarrow E .
$$

Then $a d_{x}$ is a derivation; these are the inner

derivations from $A$ to $E$. For $\varphi \in \Phi_{A} \cup\{0\}$, a point derivation at $\varphi$ is a linear functional $d$ on $A$ such that

$$
d(a b)=\varphi(a) d(b)+\varphi(b) d(a) \quad(a, b \in A) .
$$

Let $A$ be an algebra. Then the space $A \otimes A$ is an $A$-bimodule for maps that satisfy the conditions that $a \cdot(b \otimes c)=a b \otimes c$ and $(b \otimes c) \cdot a=b \otimes c a$ for $a, b, c \in A$. There is a linear map $\pi_{A}: A \otimes A \rightarrow A$ such that $\pi_{A}(a \otimes b)=a b(a, b \in A)$. Suppose that $A$ is unital. Then a diagonal

for $A$ is an element $u \in A \otimes A$ such that $a \cdot u=u \cdot a(a \in A)$ and $\pi_{A}(u)=e_{A}$. For example, let $n \in \mathbb{N}$. Then

$$
\frac{1}{n} \sum_{i, j=1}^{n} E_{i j} \otimes E_{j i}
$$

is a diagonal for $A=\mathbb{M}_{n}$.

\section{Banach spaces}

Let $E$ be a Banach space. Then the closed unit ball of radius $r>0$ in $E$ is denoted by $E_{[r]}$. The dual space of $E$ is denoted by $E^{\prime}$, and the second dual space is $E^{\prime \prime}$; we regard $E$ as a closed subspace of $E^{\prime \prime}$. The weak-* topology on $E^{\prime}$ is denoted by $\sigma\left(E^{\prime}, E\right)$, or simply by $\sigma$ when the spaces are clear. The values of $\lambda \in E^{\prime}$ at $x \in E$ and of $\Phi \in E^{\prime \prime}$ at $\lambda \in E^{\prime}$ are denoted by $\langle x, \lambda\rangle$ and $\langle\Phi, \lambda\rangle$, respectively. The closure of a subset $X$ of $E^{\prime}$ in the weak-* topology is $\bar{X}^{\sigma}$. Let $F$ be a linear subspace of $E$. Then the annihilator

$F^{\circ}$ of $F$ in $E^{\prime}$ is

$$
F^{\circ}=\left\{\lambda \in E^{\prime}:\langle x, \lambda\rangle=0(x \in F)\right\} .
$$

Let $E$ and $F$ be Banach spaces. Then the space of all bounded linear operators from $E$ to $F$ is denoted by $\mathcal{B}(E, F) ; \mathcal{B}(E, F)$ is a Banach space with respect to the operator norm. We write $\mathcal{B}(E)$ for $\mathcal{B}(E, E)$; the dual of $T \in \mathcal{B}(E, F)$ is $T^{\prime} \in \mathcal{B}\left(F^{\prime}, E^{\prime}\right)$.

We use the notations $c_{0}, \ell^{\infty}, \ell^{p}$ for standard Banach spaces of sequences on $\mathbb{N}$ (where $p \geq 1$ ), and, for example, we write $\ell_{n}^{p}$ for the space $\mathbb{C}^{n}$ with the $\ell^{p}$-norm. 
The Banach space $\ell^{1}(S)$ Let $S$ be a non-empty set, and consider the Banach space $\ell^{1}(S)$. A generic element of $\ell^{1}(S)$ has the form

$$
f=\sum\left\{\alpha_{s} \delta_{s}: s \in S\right\}
$$

where

$$
\|f\|_{1}=\sum\left\{\left|\alpha_{s}\right|: s \in S\right\}<\infty .
$$

The dual space of $E:=\ell^{1}(S)$ is $E^{\prime}=\ell^{\infty}(S)$, with the duality

$$
\langle f, \lambda\rangle=\sum\{f(s) \lambda(s): s \in S\} \quad\left(f \in E, \lambda \in E^{\prime}\right) .
$$

We shall later identify $\ell^{\infty}(S)$ with $C(\beta S)$ (see Chapter 5 for more details). The Banach space $\ell^{1}(S)$ is identified with the dual space of $c_{0}(S)$, with the above duality.

Let $S$ and $T$ be non-empty sets. The projective tensor product $\ell^{1}(S) \widehat{\otimes} \ell^{1}(T)$ is identified with $\ell^{1}(S \times T)$ by setting

$$
(f \otimes g)(s, t)=f(s) g(t) \quad(s \in S, t \in S)
$$

for $f \in \ell^{1}(S)$ and $g \in \ell^{1}(T)$. Note that, for an element $F=\sum \alpha_{i j} \delta_{\left(s_{i}, t_{j}\right)}$ in $\ell^{1}(S) \widehat{\otimes} \ell^{1}(T)$, where $\left\{\left(s_{i}, t_{j}\right): i, j \in \mathbb{N}\right\}$ is a set of distinct points in $S \times T$, we have

$$
\|F\|_{\pi}=\|F\|_{1}=\sum_{i, j=1}^{\infty}\left|\alpha_{i j}\right| .
$$

Continuous functions and measures Throughout, a locally compact space is assumed to be Hausdorff, unless we say otherwise.

Let $\Omega$ be a non-empty, locally compact space. Then we denote by $C(\Omega)$ the algebra (for the pointwise product) of all continuous functions on $\Omega$. The support

of $f \in C(\Omega)$ is the set

$$
\text { supp } f=\overline{\{x \in \Omega: f(x) \neq 0\}} \text {. }
$$

We denote by $C B(\Omega)$ the algebra of bounded, continuous functions on $\Omega$, by $C_{00}(\Omega)$ the subalgebra of $C B(\Omega)$ consisting of functions of compact support, and by $C_{0}(\Omega)$ the subalgebra of $C B(\Omega)$ of functions that vanish at infinity. The uniform norm

on $\Omega$ is denoted by $|\cdot|_{\Omega}$, so that $\left(C B(\Omega),|\cdot|_{\Omega}\right)$ and $\left(C_{0}(\Omega),|\cdot|_{\Omega}\right)$ are uniform algebras,

as in $[\mathbf{1 9}]$. The space of real-valued functions in $C(\Omega)$ is denoted by $C_{\mathbb{R}}(\Omega)$, etc.

The space consisting of all complex-valued,

regular Borel measures 
on $\Omega$ is denoted by $M(\Omega)$; the space of real-valued measures in $M(\Omega)$ is $M_{\mathbb{R}}(\Omega)$, and the cone of positive measures is $M(\Omega)^{+}$. For a Borel subset $B$ of $\Omega$ and $\mu \in M(\Omega)$, we denote the restriction measure by $\mu \mid B$. Let $\mu \in M(\Omega)$. Then $|\mu|$ is the total variation measure corresponding to $\mu$, and so $|\mu| \in M(\Omega)^{+}$. By the Jordan decomposition theorem, each $\mu \in M(\Omega)$ is a linear combination of four measures in $M(\Omega)^{+}$. The support

of $\mu$, denoted by supp $\mu$, is the complement of the maximal open subset $U$ of $\Omega$ such that $|\mu|(U)=0$.

The space $M(\Omega)$ is a Banach space with respect to the total variation norm $\|\cdot\|$, so that

$$
\|\mu\|=|\mu|(\Omega) \quad(\mu \in M(\Omega) .
$$

The Banach space $(M(\Omega),\|\cdot\|)$ is identified with the dual space of $C_{0}(\Omega)$ via the duality

$$
\langle\mu, \lambda\rangle=\int_{\Omega} \lambda(s) \mathrm{d} \mu(s) \quad\left(\mu \in M(\Omega), \lambda \in C_{0}(\Omega)\right)
$$

(see $[\mathbf{1 9}])$.

Let $\mu \in M(\Omega)^{+}$, and let $K$ be a compact subspace of $\Omega$. Recall that

$$
\mu(K)=\inf \left\{\langle\mu, \lambda\rangle: \lambda \in C_{00}(\Omega), \lambda \geq 0, \lambda \mid K=1\right\}
$$

In the case where $\Omega$ is a compact space, $\|\mu\|=\mu(\Omega)=\langle\mu, 1\rangle$, where 1 denotes the function constantly equal to 1 on $\Omega$.

Let $x \in \Omega$. Then we identify $x$ with $\delta_{x} \in M(\Omega)^{+}$, where $\delta_{x}$ is the point mass at $x$. It is standard that $\operatorname{lin}\{x: x \in \Omega\}$ is weak-* dense in $M(\Omega)$. A measure $\mu \in M(\Omega)$ is discrete

if there is a countable set $E$ such that $|\mu|(\Omega \backslash E)=0$; we identify the closed subspace of $M(\Omega)$ consisting of the discrete measures with $\ell^{1}(\Omega)$. A measure $\mu \in M(\Omega)$ is continuous if $\mu(\{x\})=0 \quad(x \in \Omega)$; the closed subspace of $M(\Omega)$ consisting of the continuous measures is denoted by $M_{c}(\Omega)$. The discrete and continuous components of $\mu \in$ $M(\Omega)$ are denoted by $\mu_{d}$ and $\mu_{c}$, respectively; we have $\|\mu\|=\left\|\mu_{d}\right\|+$ $\left\|\mu_{c}\right\|$ for each $\mu \in M(\Omega)$, and so $M(\Omega)=\ell^{1}(\Omega) \oplus M_{c}(\Omega)$ as an $\ell^{1}$-direct sum of Banach spaces.

Let $K$ be a compact subspace of $\Omega$. Then

$$
I(K)=\left\{\lambda \in C_{0}(\Omega): \lambda \mid K=0\right\}
$$


so that $I(K)$ is a closed ideal in $C_{0}(\Omega)$. We shall identify $M(K)$ with $I(K)^{\circ}$, so that

$$
\begin{aligned}
M(K) & =\{\mu \in M(\Omega):\langle\mu, \lambda\rangle=0 \quad(\lambda \in I(K))\} \\
& =\{\mu \in M(\Omega):|\mu|(\Omega \backslash K)=0\} .
\end{aligned}
$$

The subspace $M(K)$ is a weak-* closed subspace of $M(\Omega)$. For each $\mu \in M(\Omega)$, we have $\mu \mid K \in M(K)$ and $\mu \mid(\Omega \backslash K) \in M(\Omega \backslash K)$, and $\|\mu\|=\|\mu|K\|+\| \mu|(\Omega \backslash K)\|$. Thus

$$
M(\Omega)=M(K) \oplus M(\Omega \backslash K)
$$

as an $\ell^{1}$-direct sum of Banach spaces.

Let $S$ be a non-empty set, and let $E=\ell^{1}(S)$, so that the dual of $\ell^{\infty}(S)$ is $E^{\prime \prime}=M(\beta S)$. Set $S^{*}=\beta S \backslash S$, a closed subspace of $\beta S$, called the growth

of $S$. We note that the relative weak-* topology on $M\left(S^{*}\right)$ from $\sigma$ is the same as the topology $\sigma\left(M\left(S^{*}\right), C\left(S^{*}\right)\right)$, and that $\overline{\ell^{1}\left(S^{*}\right)} \sigma=M\left(S^{*}\right)$.

\section{Banach algebras}

For the theory of Banach algebras, see [19]. For example, $\mathcal{B}(E)$ is a Banach algebra for each Banach space $E$, and $\left(C(\Omega),|\cdot|_{\Omega}\right)$ is a Banach algebra for each compact space $\Omega$.

We wish to note a specific convention of the present memoir; it is different from that in [19]. A Banach algebra is an algebra $A$ which is also a Banach space for a norm $\|\cdot\|$ and is such that

$$
\|a b\| \leq\|a\|\|b\| \quad(a, b \in A) .
$$

Now suppose that $A$ has an identity $e_{A}$; in distinction from [19] and some other sources, we do not require that $\left\|e_{A}\right\|=1$.

We make the following presumably well-known remark about the norms of identities in Banach algebras.

Proposition 1.1. Let I be a closed ideal in a Banach algebra $A$. Suppose that $A / I$ and $I$ have identities $e_{A / I}$ and $e_{I}$, respectively, with $\left\|e_{A / I}\right\|=\alpha$ and $\left\|e_{I}\right\|=\beta$. Then $A$ has an identity $e_{A}$, and

$$
\left\|e_{A}\right\| \leq \alpha+\beta+\alpha \beta \text {. }
$$

Proof. Write $q: A \rightarrow A / I$ for the quotient map. We have remarked that $e_{I} \in \mathfrak{Z}(A)$. Take $\varepsilon>0$. Then there exists $a_{0} \in A$ with $q\left(a_{0}\right)=e_{A / I}$ and $\left\|a_{0}\right\| \leq \alpha+\varepsilon$.

Consider the element $e=e_{I}+a_{0}-a_{0} e_{I} \in A$. Then $q(e)=e_{A / I}$ because $q\left(e_{I}\right)=0$. For each $a \in A$, we have $a e-a=e a-a \in I$, and 
SO

$$
a e-a=e_{I}(a e-a)=e_{I} a e_{I}+e_{I} a a_{0}-a a_{0} e_{I}-e_{I} a=0 .
$$

Thus $e$ is the unique identity of $A$. We have $\|e\| \leq \alpha+\beta+(\alpha+\varepsilon) \beta+\varepsilon$. This is true for each $\varepsilon>0$, and so $\left\|e_{A}\right\| \leq \alpha+\beta+\alpha \beta$.

Let $(A,\|\cdot\|)$ be a Banach algebra. Then each maximal modular left ideal of $A$ is closed, the radical $R_{A}$ is a closed ideal in $A$, and $A / R_{A}$ is a semisimple Banach algebra, but in general neither $Q_{A}$ nor $N_{A}$ is $\|\cdot\|$ closed in $A$. Again neither $Q_{A}$ nor $N_{A}$ is necessarily closed under either addition or multiplication in $A$. In the case where $A$ is commutative, we have

$$
N_{A} \subset Q_{A}=R_{A}=\bigcap\left\{\operatorname{ker} \varphi: \varphi \in \Phi_{A}\right\} .
$$

However we may have the equality $R_{A}=Q_{A}$ even when $A$ is not commutative. For example, let $\mathbb{S}_{2}$ denote the free semigroup on 2 generators,

as in Example 3.42, below, and set $A=\ell^{1}\left(\mathbb{S}_{2}\right)$ with convolution product, a non-commutative Banach algebra. Then $R_{A}=Q_{A}=\{0\}$ [19, Theorem 2.3.14].

Let $A$ be a Banach algebra. A left approximate identity

in $A$ is a net $\left(e_{\alpha}\right)$ in $A$ such that $\lim _{\alpha} e_{\alpha} a=a(a \in A)$; a bounded left approximate identity in $A$ is a left approximate identity $\left(e_{\alpha}\right)$ such that $\sup _{\alpha}\left\|e_{\alpha}\right\|<\infty$. Similarly, we define a [bounded] right approximate identity in $A$. A [bounded] approximate identity in $A$ is a net which is both a [bounded] left approximate identity and a [bounded] right approximate identity. A Banach algebra which has a bounded left approximate identity and a bounded right approximate identity has a bounded approximate identity. By Cohen's factorization theorem $[\mathbf{1 9}$, Corollary 2.9.30(i)], a Banach algebra with a bounded left approximate identity or a bounded right approximate identity factors.

The Banach algebra $A$ is essential if $\overline{A^{2}}=A$.

Let $(A,\|\cdot\|)$ be a Banach algebra. For each $a \in A$, the spectrum $\sigma_{A}(a)$ of $a$ is non-empty and compact. The spectral radius of $a$ is denoted by $\nu_{A}(a)$ or $\nu(a)$; we have

$$
\nu_{A}(a)=\lim _{n \rightarrow \infty}\left\|a^{n}\right\|^{1 / n}=\sup \{|z|: z \in \sigma(a)\} .
$$

Suppose that $A$ is unital, that $B$ is a unital closed subalgebra of $A$, and that $a \in B$. Then

$$
\partial \sigma_{B}(a) \subset \sigma_{A}(a) \subset \sigma_{B}(a) \subset \widehat{\sigma_{A}(a)},
$$


where $\widehat{X}$ denotes the union of $X$ and the bounded components of $\mathbb{C} \backslash X$ for a compact plane set $X$, and $\partial X$ denotes the topological frontier of $X$ with respect to $\mathbb{C}$.

Each character on a Banach algebra is continuous, and the character space $\Phi_{A}$ of a Banach algebra $A$ is a locally compact space for the relative topology $\sigma\left(A^{\prime}, A\right)$; the Gel'fand transform

$$
\mathcal{G}: A \rightarrow C_{0}\left(\Phi_{A}\right)
$$

is a continuous homomorphism when $\Phi_{A} \neq \emptyset$. We always have the inclusion $R_{A} \subset \operatorname{ker} \mathcal{G}$; if, further, $A$ is commutative, then $R_{A}=\operatorname{ker} \mathcal{G}$. When $A$ is a commutative $C^{*}$-algebra, the Gel'fand transform is a surjection, and so $A \cong C_{0}\left(\Phi_{A}\right)$.

Let $I$ be a closed ideal in a Banach algebra $A$, and let $q: A \rightarrow A / I$ be the quotient map. Then $q^{\prime} \mid \Phi_{A / I}: \Phi_{A / I} \rightarrow \Phi_{A}$ is a continuous embedding.

Let $A$ and $B$ be Banach algebras. The projective tensor product $\left(A \widehat{\otimes} B,\|\cdot\|_{\pi}\right)$

of $A$ and $B$ is defined on [19, p. 165]; $\left(A \widehat{\otimes} A,\|\cdot\|_{\pi}\right)$ is itself a Banach algebra. The map $\pi_{A}: A \widehat{\otimes} A \rightarrow A$ is a continuous linear map such that

$$
\pi_{A}(a \otimes b)=a b \quad(a, b \in A)
$$

$\pi$ is a homomorphism of algebras when $A$ is commutative.

Let $E$ be a Banach space. Then we regard $\mathbb{M}_{m, n}(E)$ as a Banach space by taking the norm to be specified by

$$
\left\|\left(x_{i j}\right)\right\|=\sum\left\{\left\|x_{i j}\right\|: i \in \mathbb{N}_{m}, j \in \mathbb{N}_{n}\right\} \quad\left(\left(x_{i j}\right) \in \mathbb{M}_{m, n}(E)\right) .
$$

In the case where $A$ is a Banach algebra, the algebra $\mathbb{M}_{n}(A)$ is a Banach algebra with respect to this norm; we have $\left\|I_{n}\right\|=n$. However we note that this norm is different from that given in [19, Example 2.1.18(ii)]; if we identify $\mathbb{M}_{n}$ with $\mathcal{B}\left(\ell_{n}^{p}\right)$, say, where $p \geq 1$, the norm $\|\cdot\|_{p}$ on $\mathbb{M}_{n}$ is such that $\left\|I_{n}\right\|_{p}=1$.

The Banach algebras $L^{1}(G)$ and $M(G)$ Let $G$ be a locally compact group.

Then we define the group algebra $L^{1}(G)$ (using the left Haar measure) and the measure algebra $M(G)$ as in $[\mathbf{1 9}]$;

we recall that the product of $\mu, \nu \in M(G)$ is specified by the formula

$$
\langle\mu \star \nu, \lambda\rangle=\int_{G} \int_{G} \lambda(s t) \mathrm{d} \mu(s) \mathrm{d} \nu(t) \quad\left(\lambda \in C_{0}(G)\right),
$$

so that $\delta_{s} \star \delta_{t}=\delta_{s t}(s, t \in G)$. It is standard that $L^{1}(G)$ is a closed ideal in $M(G)$, identified with the space $M_{a c}(G)$ of measures which are 
absolutely continuous with respect to the left Haar measure on $G$. The closed subspace $\ell^{1}(G)$ is a Banach subalgebra of $M(G)$, and $M_{c}(G)$ is a closed ideal in $M(G)$, and so

$$
M(G)=\ell^{1}(G) \ltimes M_{c}(G)
$$

as a semi-direct product; the quotient map $M(G) \rightarrow \ell^{1}(G)$ is denoted by $q_{G}$, so that

$$
q_{G}:(M(G), \star) \rightarrow\left(\ell^{1}(G), \star\right)
$$

is a continuous epimorphism. See [19, Chapter 3.3] and [72] for details of these algebras.

Summary In Chapter 2, we shall recall the definitions of some basic properties of Banach algebras, concentrating on the notions of amenability and weak amenability; we shall define the amenability constant of a Banach algebra. As an example, we shall discuss Munn algebras. We shall define the two Arens products, $\square$ and $\diamond$, on the second dual $A^{\prime \prime}$ of a Banach algebra $A$, and the topological centres of $A^{\prime \prime}$.

In Chapter 3, we shall give some basic properties of semigroups; as an example, we shall discuss Rees semigroups and the structure of some semigroups in detail. We shall prove results about almost left disjoint subsets of a semigroup; these results will be required later. We shall recall the definitions of various (compact) topological semigroups, and conclude with a collection of examples of semigroups.

In Chapter 4, we shall move to the definitions of the semigroup algebra $\ell^{1}(S)$ of such a semigroup $S$, commenting on the semi-character space of $S$, and proving some results on the spectra of elements in $\ell^{1}(S)$. In particular, we shall describe the semigroup algebra of a Rees semigroup, giving some new calculations on the norms of the identity of $\ell^{1}(S)$, etc.

In Chapter 5, we shall recall some facts about the Stone-Čech compactification $\beta S$ of a set $S$, and in Chapter 6 we shall note that, for a semigroup $S, \beta S$ is also a semigroup with respect to two products, which we denote by $\square$ and $\diamond$. We shall consider the second dual space, $\ell^{1}(S)^{\prime \prime}$, of a semigroup algebra $\ell^{1}(S)$, identifying $\ell^{1}(S)^{\prime \prime}$ with $M(\beta S)$, the Banach space of measures on $\beta S$. Thus there are two products, and $\diamond$, on $M(\beta S)$. We regard $(\beta S, \square)$ and $(\beta S, \diamond)$ as subsemigroups of the multiplicative semigroups of $(M(\beta S), \square)$ and $(M(\beta S), \diamond)$, respectively. We have results, some new, about subsemigroups and ideals in the semigroups $(\beta S, \square)$. In particular, we shall obtain in Theorem 
6.46, the perhaps surprising result that $\overline{\mathbb{N}_{[k+1]}^{*}} \subsetneq \overline{\mathbb{N}^{*} \square \overline{\mathbb{N}_{[k]}^{*}}}$ for each $k \in \mathbb{N}$ with $k \geq 2$.

In Chapter 7 , we shall study the Banach algebras $(M(\beta S), \square)$ and $(M(\beta S), \diamond)$ for a semigroup $S$. In fact, we have a somewhat more general theory that arises from that of introverted $C^{*}$-subalgebras of $\ell^{\infty}(S)$. We have many results on the closed ideals in $(M(\beta S), \square)$, and we have some results on nilpotent elements and radicals in $\ell^{1}(\beta S)$ and $(M(\beta S), \square)$, but we are not able to determine when $\ell^{1}(\beta S)$ is semisimple, or to characterize the radical of $(M(\beta S), \square)$. We shall make some remarks on the semi-character space $\Phi_{\beta S}$ of the semigroup $\beta S$; we note that $\Phi_{\beta S}$ is the character space of the semigroup algebra $\ell^{1}(\beta S)$. We shall conclude the chapter with a collection of examples.

In Chapter 8, we shall introduce some spaces related to $\ell^{\infty}(S)$ and $L^{1}(G)$, where $S$ is a semigroup and $G$ is a locally compact group. For example, these spaces involve weakly almost periodic and uniformly continuous functions.

In Chapter 9, we shall discuss amenability for semigroups, and, in particular, the supports of left-invariant means. Some results are special to the case where our semigroup is $(\mathbb{N},+)$.

In Chapter 10, we shall determine exactly when a semigroup algebra $\ell^{1}(S)$ is amenable, so answering an open question; the results involve Rees semigroups with a zero. This will lead us to a discussion of the amenability constant $C_{S}$ of such a semigroup algebra; for example, we shall prove in Theorem 10.27 that $S$ is a group whenever $C_{S}<5$.

Chapter 11 addresses the amenability and weak amenability of the Banach algebras $(M(\beta S), \square)$ and $(M(\beta S), \diamond)$. In Theorem 11.8, we shall prove that, for each infinite semigroup $S$, these Banach algebras are not amenable, and in Theorem 11.15 that, for each infinite, cancellative semigroup $S$, the algebra $M(\beta S)$ is not even weakly amenable. This allows us to resolve a further open question by proving in Theorem 11.17 that the second dual algebra $\left(L^{1}(G)^{\prime \prime}, \square\right)$ is weakly amenable if and only if $G$ is finite.

Finally, in Chapter 12, we shall extend known results on the topological centres of the Banach algebras $\ell^{1}(S)$ and the semigroup $(\beta S, \square)$ for various semigroups $S$. The strongest result is Theorem 12.15, which asserts that, for a class of semigroups that includes all cancellative semigroups, there are two elements $a$ and $b$ in the growth $S^{*}$ of $S$ with the property that each $\mu \in M(\beta S, \square)$ such that $\mu \square a=\mu \diamond a$ and $\mu \square b=\mu \diamond b$ is necessarily in $\ell^{1}(S)$. We discuss the further properties that such elements $a$ and $b$ can be required to possess. We shall also 
obtain a similar result for more general semigroups and for the algebra $L U C(G)^{\prime}$.

The memoir concludes with a list of selected open problems that we have not been able to resolve.

This memoir was submitted in September 2006; some corrections, extra examples, and updated references were added after acceptance of the manuscript in August 2008.

Acknowledgements We are grateful to John Duncan, Neil Hindman, and Yevhen Zelenyuk for very helpful contributions at points of this memoir. We are grateful to Andreas Blass, Hossein Esslamzadeh, Mahmoud Filali, Fereidoun Ghahramani, Imre Leader, Rick Loy, Salvador Hernàndez Muñoz, Matthias Neufang, Michael White, and George Willis for valuable comments and references. We are also very grateful to the referee for a careful reading of the manuscript, for raising some interesting points, and for suggesting some extra references.

We acknowledge with thanks the financial support of NSERC grant A7679 to A. T.-M. Lau and an LMS travel grant to D. Strauss. 



\section{CHAPTER 2}

\section{Banach algebras and their second duals}

In this chapter, we shall recall some standard properties of Banach algebras and their second duals, and prove results that we shall use later. In particular, we shall recall the definitions of amenable, weakly amenable, and approximately amenable Banach algebras, and shall be concerned with the amenability constant of an amenable Banach algebra. For a substantial account of Banach algebra theory, see [19].

Amenability and weak amenability Let $A$ be a Banach algebra, and let $(E,\|\cdot\|)$ be a Banach space which is also an $A$-bimodule. Then $E$ is a Banach A-bimodule

if

$$
\|a \cdot x\| \leq\|a\|\|x\|, \quad\|x \cdot a\| \leq\|a\|\|x\| \quad(a \in A, x \in E) .
$$

For example, $E=A$ is a Banach $A$-bimodule with module operations the product $m_{A}$. The dual of a Banach $A$-bimodule

$E$ is the Banach space $E^{\prime}$ with the operations defined by

$\langle x, a \cdot \lambda\rangle=\langle x \cdot a, \lambda\rangle, \quad\langle x, \lambda \cdot a\rangle=\langle a \cdot x, \lambda\rangle \quad\left(a \in A, x \in E, \lambda \in E^{\prime}\right)$.

For example, $A^{\prime}$ and $A^{\prime \prime}$ are Banach $A$-bimodules. The Banach space $\left(A \widehat{\otimes} A,\|\cdot\|_{\pi}\right)$ is a Banach $A$-bimodule for continuous maps satisfying the conditions that $a \cdot(b \otimes c)=a b \otimes c$ and $(b \otimes c) \cdot a=b \otimes c a$ for $a, b, c \in A$.

Definition 2.1. Let $A$ be a Banach algebra. Then $A$ is amenable if each continuous derivation from $A$ into each dual Banach A-bimodule is inner, and weakly amenable if each continuous derivation from $A$ into the specific dual Banach A-bimodule $A^{\prime}$ is inner.

We shall later require the following well-known facts about amenability and weak amenability. For the proofs of clauses (i)-(viii), see [19]; in particular, clause (viii) is [19, Theorem 2.9.58(ii)] with 'left' and 'right' exchanged. For the proof of (x), see [96, Lemma 2.3].

Proposition 2.2. (i) A Banach algebra $A$ is amenable if and only if $A^{\#}$ is amenable. 
(ii) Each amenable Banach algebra has a bounded approximate identity, and hence factors.

(iii) Let $A$ be a weakly amenable Banach algebra. Then $A$ is essential and there are no non-zero, continuous point derivations on $A$.

(iv) Let $A$ be a commutative, weakly amenable Banach algebra. Then every continuous derivation from $A$ into a commutative Banach A-bimodule is zero.

(v) Each commutative Banach algebra that is spanned by its idempotents is weakly amenable.

(vi) Let $A$ be a Banach algebra with a closed ideal I. Suppose that $I$ and $A / I$ are both amenable (respectively, weakly amenable). Then $A$ is amenable (respectively, weakly amenable).

(vii) Let $A$ be an amenable Banach algebra with a closed ideal $I$. Then A/I is amenable. Suppose, further, that I is complemented in $A$ as a Banach space. Then I is amenable, I has a bounded approximate identity, and I factors.

(viii) Let $A$ be an amenable Banach algebra with a closed left ideal I. Suppose that I is complemented in $A$ as a Banach space. Then I has a bounded right approximate identity.

(ix) Let $A$ be a Banach algebra, and let I be a closed ideal, with quotient map $\pi: A \rightarrow A / I$. Suppose that $d$ is a non-zero, continuous point derivation at $\varphi \in \Phi_{A / I}$. Then $d \circ \pi$ is a non-zero, continuous point derivation at $\varphi \circ \pi \in \Phi_{A}$.

(x) Let $A=B \ltimes I$ be a Banach algebra, where $B$ is a closed subalgebra and $I$ is a closed ideal in $A$. Suppose that $A$ is weakly amenable. Then $B$ is weakly amenable.

The next definition is the Banach-algebra analogue of the notion of a diagonal.

Definition 2.3. Let $A$ be a Banach algebra. A bounded approximate diagonal

for $A$ is a bounded net $\left(u_{\alpha}\right)$ in $\left(A \widehat{\otimes} A,\|\cdot\|_{\pi}\right)$ such that

$$
\lim _{\alpha}\left\|a \cdot u_{\alpha}-u_{\alpha} \cdot a\right\|_{\pi}=0 \quad(a \in A)
$$

and

$$
\lim _{\alpha}\left\|\pi_{A}\left(u_{\alpha}\right) a-a\right\|=0 \quad(a \in A) .
$$

Now take $C \geq 1$. Then $A$ is $C$-amenable

if $A$ has a bounded approximate diagonal $\left(u_{\alpha}\right)$ such that $\sup _{\alpha}\left\|u_{\alpha}\right\|_{\pi} \leq$ $C$. The amenability constant, 
$A M(A)$, of $A$ (in the case where $A$ is amenable) is the infimum of the numbers $C$ such that $A$ is $C$-amenable.

$A$ virtual diagonal

for $A$ is an element $\mathrm{M}$ of $(A \widehat{\otimes} A)^{\prime \prime}$ such that, for each $a \in A$, we have $a \cdot \mathrm{M}=\mathrm{M} \cdot a$ and $\pi_{A}^{\prime \prime}(\mathrm{M}) \cdot a=a$.

Let $A$ be a Banach algebra with an identity. We may suppose that $\left\|e_{A}\right\|=1$ and that each bounded approximate diagonal $\left(u_{\alpha}\right)$ is such that $\pi_{A}\left(u_{\alpha}\right)=e_{A}$ for each $\alpha$.

It is a well-known theorem of Johnson $([\mathbf{8 4}]$; see $[\mathbf{1 9}$, Theorem 2.9.65]) that a Banach algebra $A$ is amenable if and only if $A$ has a bounded approximate diagonal if and only if $A$ has a virtual diagonal, and hence $A$ is amenable if and only if $A$ is $C$-amenable for some $C \geq 1$. Indeed, $A$ is $C$-amenable if and only if there is a virtual diagonal $\mathrm{M}$ in $(A \widehat{\otimes} A)_{[C]}^{\prime \prime}$.

The notion of $C$-amenability comes from [84] (see also [125, §2.3]), and the amenability constant $A M(A)$ was specifically introduced in [86]. Clearly $A M(A)$ is actually the minimum of the appropriate constants $C$, and $A M(A) \geq 1$. Although we shall not use the fact, we remark that each amenable $C^{*}$-algebra $A$ has $A M(A)=1[\mathbf{7 0}$, Theorem 3.1].

Proposition 2.4. Let $A$ be an amenable Banach algebra with an identity $e_{A}$.

(i) $A M(A) \geq\left\|e_{A}\right\|$.

(ii) Let $I$ be a closed ideal with an identity $e_{I}$. Then $I$ is amenable, with $A M(I) \leq\left\|e_{I}\right\| A M(A)$.

Proof. (i) This is immediate.

(ii) Let $\left(u_{\alpha}\right)$ be a bounded approximate diagonal for $A$ in $A \widehat{\otimes} A$ with $\sup _{\alpha}\left\|u_{\alpha}\right\|_{\pi} \leq A M(A)$. For each $\alpha$, set

$$
v_{\alpha}=e_{I} \cdot u_{\alpha} \cdot e_{I} \in I \widehat{\otimes} I .
$$

Then $\pi_{I}\left(v_{\alpha}\right)=e_{I} e_{A} e_{I}=e_{I}$ and

$$
\lim _{\alpha}\left\|a \cdot v_{\alpha}-v_{\alpha} \cdot a\right\|_{\pi} \leq\left\|e_{I}\right\|^{2} \lim _{\alpha}\left\|a \cdot u_{\alpha}-u_{\alpha} \cdot a\right\|_{\pi}=0 \quad(a \in I) .
$$

Also,

$$
\lim _{\alpha}\left\|v_{\alpha}-e_{I} \cdot u_{\alpha}\right\|_{\pi} \leq \lim _{\alpha}\left\|e_{I}\right\|\left\|u_{\alpha} \cdot e_{I}-e_{I} \cdot u_{\alpha}\right\|_{\pi}=0,
$$

and so $\lim \sup _{\alpha}\left\|v_{\alpha}\right\| \leq\left\|e_{I}\right\| A M(A)$. This shows that $I$ is amenable and that $A M(I) \leq\left\|e_{I}\right\| A M(A)$. 
For $n \in \mathbb{N}$, let $A_{n}$ be the Banach algebra $\left(\ell_{n}^{1}, \cdot\right)$. Then the identity of $A_{n}$ is $\delta_{1}+\cdots+\delta_{n}$, with norm $n$; the unique diagonal for $A$ is $u=\sum_{i=1}^{n} \delta_{i} \otimes \delta_{i}$, and $\|u\|_{\pi}=n$, so that $A M\left(A_{n}\right)=n$.

Again let $A=A_{D}$ be the space $\ell_{2}^{1}$ with product specified by

$$
\left(z_{1}, w_{1}\right)\left(z_{2}, w_{2}\right)=\left(z_{1} z_{2}, z_{1} w_{2}+z_{2} w_{1}+w_{1} w_{2}\right) \quad\left(z_{1}, z_{2}, w_{1}, w_{2} \in \mathbb{C}\right),
$$

so that $A$ is a Banach algebra and $A_{D}$ is isomorphic to $A_{2}$, specified above. The identity of $A$ is $e_{A}=(1,0)$, and $\left\|e_{A}\right\|_{1}=1$. Suppose that

$\Delta=\alpha(1,0) \otimes(1,0)+\beta(1,0) \otimes(0,1)+\gamma(0,1) \otimes(1,0)+\delta(0,1) \otimes(0,1)$ is a diagonal for $A$. Since $\pi_{A}(\Delta)=(1,0)$, we see that $\alpha=1$ and $\beta+\gamma+\delta=0$. Since $(0,1) \cdot \Delta=\Delta \cdot(0,1)$, we have $\beta=\gamma=-\alpha=-1$, and then $\delta=2$. Thus the unique diagonal of $A$ is

$$
\Delta=(1,0) \otimes(1,0)-(1,0) \otimes(0,1)-(0,1) \otimes(1,0)+2(0,1) \otimes(0,1),
$$

and so $A M\left(A_{D}\right)=5$.

Note that the amenability constant $A M(A)$ for a Banach algebra $A$ depends on the actual norm on $A$, as the above calculation shows. For a further example, set $A=\mathbb{M}_{n}$. Then a diagonal for $A$ is

$$
\Delta=\frac{1}{n} \sum\left\{E_{i j} \otimes E_{j i}: i, j \in \mathbb{N}_{n}\right\}
$$

with our specified norm (of equation (1.4)) on $A$, we have $\|\Delta\|_{\pi}=n$ and $A M(A) \geq\left\|I_{n}\right\|=n$, and so $A M(A)=n$. However, when $A$ has the norm that arises from identifying $\mathbb{M}_{n}$ with $\mathcal{B}\left(\ell_{n}^{p}\right)$, where $p \geq 1$, then $A M(A)=\|\Delta\|_{\pi}=1$ [125, Example 2.3.16]. 10.

For further examples concerning amenability constants, see Chapter

Proposition 2.5. Let $A$ be a $C$-amenable Banach algebra, and let $B$ be a Banach algebra such that $\theta: A \rightarrow B$ is a continuous homomorphism with dense range. Then $B$ is $\|\theta\|^{2} C$-amenable.

Proof. There is a continuous linear $A$-module homorphism

$$
\theta \otimes \theta: A \widehat{\otimes} A \rightarrow B \widehat{\otimes} B .
$$

Suppose that $\left(u_{\alpha}\right)$ is a bounded approximate diagonal for $A$ such that $\sup _{\alpha}\left\|u_{\alpha}\right\|_{\pi} \leq C$. Set $U_{\alpha}=(\theta \otimes \theta)\left(u_{\alpha}\right)$. Then $\left(U_{\alpha}\right)$ is a bounded approximate diagonal for $B$ with $\sup _{\alpha}\left\|U_{\alpha}\right\|_{\pi} \leq\|\theta\|^{2} C$.

Corollary 2.6. Let $A$ be a $C$-amenable Banach algebra, and let $I$ be a closed ideal in $A$. Then $A / I$ is $C$-amenable. 
Amenability and weak amenability of $\mathbb{M}_{n}(A)$ Let $A$ be a Banach algebra, let $n \in \mathbb{N}$, and set $\mathfrak{A}=\mathbb{M}_{n}(A)$; further, let $E$ be a Banach $A$-bimodule, and set $\mathfrak{E}=\mathbb{M}_{n}(E)$. We shall regard $\mathfrak{E}$ as a Banach $\mathfrak{A}$-bimodule in the obvious way, so that

$$
(a \cdot x)_{i j}=\sum_{k=1}^{n} a_{i k} \cdot x_{k j}, \quad\left(a=\left(a_{i j}\right) \in \mathfrak{A}, x=\left(x_{i j}\right) \in \mathfrak{E}\right) .
$$

We identify $\mathfrak{E}^{\prime}$ with $\mathbb{M}_{n}\left(E^{\prime}\right)$, using the duality

$$
\langle a, \Lambda\rangle=\sum_{i, j=1}^{n}\left\langle a_{i j} \lambda_{i j}\right\rangle \quad\left(a=\left(a_{i j}\right) \in \mathfrak{A}, \Lambda=\left(\lambda_{i j}\right) \in \mathfrak{E}^{\prime}\right) .
$$

We note that

$$
(a \cdot \Lambda)_{i j}=\sum_{k=1}^{n} a_{j k} \cdot \lambda_{i k} \quad \text { and } \quad(\Lambda \cdot a)_{i j}=\sum_{k=1}^{n} \lambda_{k j} \cdot a_{k i}
$$

for $a=\left(a_{i j}\right) \in \mathfrak{A}$ and $\Lambda=\left(\lambda_{i j}\right) \in \mathfrak{E}^{\prime}$.

Let $D: A \rightarrow E^{\prime}$ be a continuous derivation, and define $\mathfrak{D}: \mathfrak{A} \rightarrow \mathfrak{E}^{\prime}$ by setting $\mathfrak{D}(a)_{i j}=\left(D\left(a_{j i}\right)\right)$, where we note the transposition of $i$ and $j$. Then $\mathfrak{D}$ is a continuous linear operator, and we claim that $\mathfrak{D}$ is a derivation. For this, we must verify that

$$
\langle c, \mathfrak{D}(a b)\rangle=\langle c a, \mathfrak{D} b\rangle+\langle b c, \mathfrak{D} a\rangle \quad(a, b, c \in \mathfrak{A}) .
$$

The left-hand side of (2.4) is

$$
\begin{aligned}
\sum_{i, j=1}^{n}\left\langle c_{i j}, D\left((a b)_{j i}\right)\right\rangle & =\sum_{i, j, k=1}^{n}\left\langle c_{i j}, D\left(a_{j k} b_{k i}\right)\right\rangle \\
& =\sum_{i, j, k=1}^{n}\left\langle c_{i j}, a_{j k} \cdot D\left(b_{k i}\right)+D\left(a_{j k}\right) \cdot b_{k i}\right\rangle \\
& =\sum_{i, j, k=1}^{n}\left(\left\langle c_{i j} a_{j k}, D\left(b_{k i}\right)\right\rangle+\left\langle b_{k i} c_{i j}, D\left(a_{j k}\right)\right\rangle\right)
\end{aligned}
$$

The term $\langle c a, \mathfrak{D} b\rangle$ on the right-hand side of (2.4) is equal to

$$
\sum_{i, j, k=1}^{n}\left\langle c_{i k} a_{k j}, D\left(b_{j i}\right)\right\rangle=\sum_{i, j, k=1}^{n}\left\langle c_{i j} a_{j k}, D\left(b_{k i}\right)\right\rangle
$$

and similarly for the term $\langle b c, \mathfrak{D} a\rangle$. Thus (2.4) is verified.

Theorem 2.7. Let $A$ be a Banach algebra, let $n \in \mathbb{N}$, and set $\mathfrak{A}=\mathbb{M}_{n}(A)$.

(i) The Banach algebra $\mathfrak{A}$ is amenable if and only if $A$ is amenable. 
(ii) Suppose that $A$ is $C$-amenable. Then $\mathfrak{A}$ is $C n$-amenable. Now suppose further that $A$ has an identity.

(iii) The Banach algebra $\mathfrak{A}$ is weakly amenable if and only if $A$ is weakly amenable.

(iv) We have $n \leq A M(\mathfrak{A}) \leq n A M(A)$.

Proof. (i) Suppose that $A$ is amenable. Then $\mathfrak{A}$ is amenable by [19, Corollary 2.9.62], a theorem of Johnson [83, Proposition 5.4]; this also follows from clause (ii), below.

Now suppose that $\mathfrak{A}$ is amenable. Let $D: A \rightarrow E^{\prime}$ be a continuous derivation, and define $\mathfrak{D}: \mathfrak{A} \rightarrow \mathfrak{E}^{\prime}$ as above. Then $\mathfrak{D}$ is a continuous derivation, and so there exists $\Lambda=\left(\lambda_{i j}\right) \in \mathfrak{E}^{\prime}$ such that

$$
\mathfrak{D}(a)=a \cdot \Lambda-\Lambda \cdot a \quad(a \in \mathfrak{A}) .
$$

Take $a \in A$, and identify $a$ with the matrix that has $a$ in the $(1,1)$ position and 0 elsewhere. Then $\lambda_{1,1} \in E^{\prime}$, and

$$
D(a)=\mathfrak{D}(a)_{1,1}=(a \cdot \lambda-\lambda \cdot a)_{1,1}=a \cdot \lambda_{1,1}-\lambda_{1,1} \cdot a \quad(a \in A),
$$

and so $D: A \rightarrow E^{\prime}$ is an inner derivation. Hence $A$ is amenable.

(ii) We shall identify $\mathfrak{A}$ with $\mathbb{M}_{n} \otimes A$, so that we can identify $\mathfrak{A} \widehat{\otimes} \mathfrak{A}$ with $\mathbb{M}_{n^{2}} \otimes(A \widehat{\otimes} A)$. For $a \in A$ and $i, j \in \mathbb{N}_{n}$, denote by $(a)_{i j}$ the element of $\mathfrak{A}$ with $a$ in the $(i, j)^{\text {th. }}$ place and 0 elsewhere, so that $\left\|(a)_{i j}\right\|=\|a\|$. For $a, b \in A$ and $i, j, k, \ell \in \mathbb{N}_{n}$, we have

$$
(a)_{i j} \otimes(b)_{k \ell}=(a \otimes b)_{i j k \ell} .
$$

Let $\left(u_{\alpha}\right)$ be a bounded approximate diagonal for $A$ with $\left\|u_{\alpha}\right\|_{\pi} \leq C$ for each $\alpha$. For each $\alpha$, we define

$$
U_{\alpha}=\frac{1}{n} \sum_{i, j=1}^{n} E_{i j} \otimes E_{j i} \otimes u_{\alpha} \in \mathfrak{A} \widehat{\otimes} \mathfrak{A} .
$$

For $c \in A$ and $r, s \in \mathbb{N}_{n}$, we have

$$
\begin{aligned}
\left(E_{r s} \otimes c\right) & \cdot U_{\alpha}-U_{\alpha} \cdot\left(E_{r s} \otimes c\right) \\
= & \frac{1}{n} \sum_{i, j=1}^{n}\left(E_{r s} E_{i j} \otimes E_{j i} \otimes c \cdot u_{\alpha}-E_{i j} \otimes E_{j i} E_{r s} \otimes u_{\alpha} \cdot c\right) \\
= & \frac{1}{n} \sum_{j=1}^{n} E_{r j} \otimes E_{j s} \otimes\left(c \cdot u_{\alpha}-u_{\alpha} \cdot c\right),
\end{aligned}
$$

and so $\left\|\left(E_{r s} \otimes c\right) \cdot U_{\alpha}-U_{\alpha} \cdot\left(E_{r s} \otimes c\right)\right\|_{\pi} \leq\left\|c \cdot u_{\alpha}-u_{\alpha} \cdot c\right\|_{\pi}$. It follows that

$$
\lim _{\alpha}\left\|\left(c_{r s}\right) \cdot U_{\alpha}-U_{\alpha} \cdot\left(c_{r s}\right)\right\|=0 \quad\left(\left(c_{r s}\right) \in \mathfrak{A}\right) .
$$


Similarly, $\lim _{\alpha}\left\|\pi_{\mathfrak{A}}\left(U_{\alpha}\right)\left(c_{r s}\right)-\left(c_{r s}\right)\right\|_{\pi}=\lim _{\alpha}\left\|\left(\pi_{A}\left(u_{\alpha}\right) c_{r s}-c_{r s}\right)\right\|_{\pi}=0$ for each $\left(c_{r s}\right) \in \mathfrak{A}$. Finally, $\sup _{\alpha}\left\|U_{\alpha}\right\|_{\pi} \leq C n$, and so $\mathfrak{A}$ is $C n$ amenable.

(iii) The same argument as the above shows that $A$ is weakly amenable whenever $\mathfrak{A}$ is weakly amenable.

Now suppose that $A$ is weakly amenable, and let $\mathfrak{D}: \mathfrak{A} \rightarrow \mathfrak{A}^{\prime}$ be a continuous derivation. We recall that we are regarding $\mathbb{M}_{n}$ as a subalgebra of $\mathfrak{A}$. Since $\mathbb{M}_{n}$ is an amenable Banach algebra, there exists an element $\Lambda=\left(\lambda_{i j}\right) \in \mathbb{M}_{n}\left(A^{\prime}\right)$ with $\mathfrak{D}\left|\mathbb{M}_{n}=a d_{\Lambda}\right| \mathbb{M}_{n}$. By replacing $\mathfrak{D}$ by $\mathfrak{D}-a d_{\Lambda}$, we may suppose that $\mathfrak{D} \mid \mathbb{M}_{n}=0$.

Next take $a \in A$. For $r, s \in \mathbb{N}_{n}$, consider the elements

$$
(a)_{r s}=E_{r s} \otimes a \in \mathfrak{A},
$$

say $\mathfrak{D}\left((a)_{r s}\right)=\left(d_{i j}^{(r, s)}(a): i, j \in \mathbb{N}_{n}\right) \in \mathbb{M}_{n}\left(A^{\prime}\right)$; set $d=d_{11}^{(1,1)}: A \rightarrow A^{\prime}$. Since $\mathfrak{D}\left(E_{r 1}\right)=\mathfrak{D}\left(E_{1 s}\right)=0$, we have

$$
\mathfrak{D}\left((a)_{r s}\right)=\mathfrak{D}\left(E_{r 1}(a)_{11} E_{1 s}\right)=E_{r 1} \cdot \mathfrak{D}\left((a)_{11}\right) \cdot E_{1 s},
$$

and so, by $(2.3), d_{i j}^{(r, s)}(a)=0\left(i, j \in \mathbb{N}_{n}\right)$ except when $(i, j)=(s, r)$ and in this case $d_{s r}^{(r, s)}(a)=d(a)$. Clearly $d: A \rightarrow A^{\prime}$ is a continuous derivation. Since $A$ is weakly amenable, there exists $\lambda \in A^{\prime}$ such that $d(a)=a \cdot \lambda-\lambda \cdot a(a \in A)$. Take $\Lambda \in \mathbb{M}_{n}\left(A^{\prime}\right)$ to be the matrix that has $\lambda$ in each diagonal position and 0 elsewhere. Then we see, again using (2.3), that

$$
\mathfrak{D}\left(\left(a_{i j}\right)\right)=\left(a_{i j}\right) \cdot \Lambda-\Lambda \cdot\left(a_{i j}\right) \quad\left(\left(a_{i j}\right) \in \mathfrak{A}\right) .
$$

This shows that $\mathfrak{A}$ is weakly amenable.

(iv) The identity $e_{\mathfrak{A}}$ of $\mathfrak{A}$ is the matrix with $e_{A}$ in each diagonal position and 0 elsewhere, and so $\left\|e_{\mathfrak{A}}\right\| \geq n$. By Proposition 2.4, we have $n \leq A M(\mathfrak{A})$. By (ii), $A M(\mathfrak{A}) \leq n A M(A)$.

Approximate amenability A variation of the notion of amenability for Banach algebras was introduced by Ghahramani and Loy in [51].

Definition 2.8. Let $A$ be a Banach algebra, and let $E$ be a Banach A-bimodule. A derivation $D: A \rightarrow E$ is approximately inner

if there is a net $\left(x_{\alpha}\right)$ in $E$ such that

$$
D a=\lim _{\alpha}\left(a \cdot x_{\alpha}-x_{\alpha} \cdot a\right) \quad(a \in A),
$$

the limit being taken in $(E,\|\cdot\|)$. The Banach algebra $A$ is approximately amenable

if, for each Banach A-bimodule E, every continuous derivation D : $A \rightarrow E^{\prime}$ is approximately inner. 
The basic properties of approximately amenable Banach algebras were established in [51]; further results are given in [55]. Certainly every amenable Banach algebra is approximately amenable; a commutative, approximately amenable Banach algebra is weakly amenable; examples of commutative, approximately amenable Banach algebras which are not amenable are given in [51, Example 6.1]. It is shown in $[26]$ that the Banach function algebras $\ell^{p}$ (for $p \geq 1$ ), which are weakly amenable, are not approximately amenable.

Characterizations of approximately amenable Banach algebras are known; they are analogous to the characterization of amenable Banach algebras as those with a bounded approximate diagonal. Proposition 2.9, below, is a modification of [51, Corollary 2.2], and Proposition 2.10 is taken from [26, Proposition 2.3].

Proposition 2.9. Let $A$ be a Banach algebra. Then $A$ is approximately amenable if and only if, for each $\varepsilon>0$ and each finite subset $S$ of $A$, there exist $F \in A \otimes A$ and $u, v \in A$ such that $\pi(F)=u+v$ and, for each $a \in S$ :

(i) $\|a \cdot F-F \cdot a+u \otimes a-a \otimes v\|_{\pi}<\varepsilon$;

(ii) $\|a-a u\|<\varepsilon$ and $\|a-v a\|<\varepsilon$.

Proposition 2.10. Let $A$ be a commutative Banach algebra. Then $A$ is approximately amenable if and only if, for each $\varepsilon>0$ and each finite subset $S$ of $A$, there exists $F \in A \otimes A$ and $u \in A$ such that $\pi(F)=2 u$, and, for each $a \in S$ :

(i) $\|a \cdot F-F \cdot a+u \otimes a-a \otimes u\|_{\pi}<\varepsilon$;

(ii) $\|a-a u\|<\varepsilon$.

For examples of commutative semigroup algebras which are approximately amenable, but not amenable, see Example 10.10.

One-dimensional extensions The construction discussed in this subsection is a general form of an important later example.

Example 2.11. Let $\left(A,\|\cdot\|_{A}\right)$ be a Banach algebra. Suppose that $p \in \mathfrak{I}(A)$ is such that $\mathbb{C} p$ is an ideal in $A$, and set $B=A / \mathbb{C} p$, with quotient norm $\|\cdot\|_{B}$ on $B$. Then we have $a p=p a=\varphi(a) p(a \in A)$ for some $\varphi \in \Phi_{A}$, so that $\varphi(p)=1$. We identify $A$ with $B \oplus \mathbb{C} p$ as a Banach space, requiring that

$$
\|a+z p\|_{A}=\|a\|_{B}+|z| \quad(a+z p \in A),
$$

and, in particular, that $\|p\|=1$. We have

$$
(a+z p)(b+w p)=a b+(\Lambda(a, b)+\varphi(a) w+\varphi(b) z+z w) p
$$


for $a, b \in B$ and $z, w \in \mathbb{C}$, where $\Lambda$ is a continuous bilinear functional on $B \times B$ such that

$$
\varphi(b) \Lambda(c, a)-\Lambda(b c, a)+\Lambda(b, c a)-\Lambda(b, c) \varphi(a)=0 \quad(a, b, c \in B) .
$$

(Here $a b$ denotes the product of $a$ and $b$ in $B$.) Since $\varphi \in \Phi_{A}$, we have

$$
\Lambda(a, b)=\varphi(a) \varphi(b)-\varphi(a b) \quad(a, b \in B) .
$$

Suppose that $B$ has an identity $e_{B}$. Then the identity of $A$ is

$$
e_{A}=e_{B}+\left(1-\varphi\left(e_{B}\right)\right) p .
$$

Set $\mathcal{A}=B \oplus \mathbb{C}$. Then $\mathcal{A}$ is a Banach algebra for the coordinatewise product and the $\ell^{1}$-norm, and the map

$$
\theta: a \mapsto(a+\mathbb{C} p, \varphi(a)), \quad A \rightarrow \mathcal{A},
$$

is an isomorphism with $\|\theta\| \leq 1$. Suppose that $B$ is $C$-amenable. Then $\mathcal{A}$ is $(C+1)$-amenable, and so, by Proposition $2.5, A$ is $\left\|\theta^{-1}\right\|^{2}(C+1)$ amenable. Always $\left\|\theta^{-1}\right\| \leq 2$, and so $A$ is $(4 C+4)$-amenable. Since we can have $\left\|\theta^{-1}\right\|=2$, this is the best estimate from this approach. Clause (ii) of the following result improves this estimate.

The ideas of this example will be developed in Chapter 4 and in Example 10.11.

TheOrem 2.12. Let $A$ and $B$ be as in Example 2.11.

(i) Suppose that $A$ is weakly amenable. Then $B$ is weakly amenable.

(ii) Suppose that $B$ is $C$-amenable. Then $A$ is $(4 C+1)$-amenable.

Proof. (i) Let $\lambda \in I^{\prime}$, where $I=\mathbb{C} p$. Set $\tau=\lambda(p) \varphi$. Then clearly $\tau \in A^{\prime}, \tau(a b)=\tau(b a) \quad(a, b \in A)$, and $\tau \mid I=\lambda$. Thus $I$ has the 'trace extension property' of [19, Definition 2.8.65]. By [19, Proposition 2.8.66(iv)], $B$ is weakly amenable.

(ii) Consider the bilinear map

$$
T:(a, b) \mapsto a \otimes b-\varphi(a) p \otimes b-\varphi(b) a \otimes p+\varphi(a) \varphi(b) p \otimes p
$$

from $B \times B$ into $A \widehat{\otimes} A$. Then $T$ is continuous, with $\|T\| \leq 4$. The corresponding map from $B \widehat{\otimes} B$ into $A \widehat{\otimes} A$ is also denoted by $T$.

Let $\mu=\varphi \otimes \varphi: B \widehat{\otimes} B \rightarrow \mathbb{C}$, so that $\|\mu\|=1$.

For $a, b \in B$, we have $p \cdot T(a, b)=T(a, b) \cdot p=0$ and

$$
\pi_{A}(T(a, b))=a b+(\Lambda(a, b)-\varphi(a) \varphi(b)) p=a b-\varphi(a b) p,
$$

so that

$$
\pi_{A}(T(u))=\pi_{B}(u)-\varphi\left(\pi_{B}(u)\right) p \quad(u \in B \widehat{\otimes} B) .
$$


Now take $a, b, c \in B$. Then

$$
\begin{aligned}
c \cdot T(a, b)= & c a \otimes b+(\Lambda(c, a)-\varphi(a) \varphi(c)) p \otimes b-\varphi(b) c a \otimes p \\
& -\Lambda(c, a) \varphi(b) p \otimes p+\varphi(a) \varphi(b) \varphi(c) p \otimes p \\
= & c a \otimes b-\varphi(c a) p \otimes b-\varphi(b) c a \otimes p+\varphi(c a) \varphi(b) p \otimes p,
\end{aligned}
$$

with a similar expression for $T(a, b) \cdot c$. Thus

$$
\begin{aligned}
c \cdot T(a, b)-T(a, b) \cdot c=c a \otimes b-a \otimes b c-p \otimes(\varphi(c a) b-\varphi(a) b c) \\
\quad+(\varphi(b c) a-\varphi(b) c a) \otimes p+(\varphi(c a) \varphi(b)-\varphi(a) \varphi(b c)) p \otimes p \\
=\quad c a \otimes b-a \otimes b c-p \cdot(c a \otimes b-a \otimes b c) \\
\quad-(c a \otimes b-a \otimes b c) \cdot p+(\varphi(c a) \varphi(b)-\varphi(a) \varphi(b c)) p \otimes p \\
=\quad x-p \cdot x-x \cdot p+\mu(x) p \otimes p,
\end{aligned}
$$

where $x=c a \otimes b-a \otimes b c$.

Since $B$ is $C$-amenable, there is a bounded approximate diagonal $\left(u_{\alpha}\right) \in(B \widehat{\otimes} B)_{[C]}$ such that $\lim _{\alpha} \pi_{B}\left(u_{\alpha}\right)=e_{B}$. For each $\alpha$, we define $U_{\alpha}=T\left(u_{\alpha}\right) \in A \widehat{\otimes} A$. Then we have $p \cdot U_{\alpha}=U_{\alpha} \cdot p=0$. Further, for each $c \in B$, we have

$$
\left\|c \cdot U_{\alpha}-U_{\alpha} \cdot c\right\|_{\pi} \leq 4\left\|c \cdot u_{\alpha}-u_{\alpha} \cdot c\right\|_{\pi},
$$

and so $\lim _{\alpha}\left\|c \cdot U_{\alpha}-U_{\alpha} \cdot c\right\|_{\pi}=0$. Finally, set

$$
V_{\alpha}=U_{\alpha}+p \otimes p \in A \widehat{\otimes} A \text {. }
$$

Then $p \cdot V_{\alpha}-V_{\alpha} \cdot p=0$ and $c \cdot V_{\alpha}-V_{\alpha} \cdot c=c \cdot U_{\alpha}-U_{\alpha} \cdot c$, so that $\lim _{\alpha}\left\|c \cdot V_{\alpha}-V_{\alpha} \cdot c\right\|_{\pi}=0$. It follows from (2.6) that

$\lim _{\alpha} \pi_{A}\left(V_{\alpha}\right)=\lim _{\alpha}\left(\pi_{B}\left(u_{\alpha}\right)-\varphi\left(\pi_{B}\left(u_{\alpha}\right)\right) p+p\right)=e_{B}+\left(1-\varphi\left(e_{B}\right) p=e_{A}\right.$,

and so the net $\left(V_{\alpha}\right)$ is a bounded approximate diagonal for $A$ such that $\left\|V_{\alpha}\right\|_{\pi} \leq 4 C+1$ for each $\alpha$. Hence $A$ is $(4 C+1)$-amenable.

We see that always $4 C+1 \leq 5 C$. We shall see in Proposition 10.18 that we can have $A M(B)=1$, but that we cannot improve on 5 for $A M(A)$, and so the above estimate is best-possible in general.

Algebras with a one-dimensional left ideal We consider the amenability and weak amenability of algebras of this form. The following result is essentially contained in [140, p. 507].

Proposition 2.13. Let $A$ be a Banach algebra with $\operatorname{dim} A \geq 2$ such that

$$
a b=\varphi(a) b \quad(a, b \in A),
$$

where $\varphi \in \Phi_{A}$. Then $A$ is weakly amenable, but not amenable. Further, $R_{A}=N_{A}=Q_{A}=\operatorname{ker} \varphi$. 
Proof. Let $D: A \rightarrow A^{\prime}$ be a continuous derivation. Then

$$
\varphi(a)\langle c, D b\rangle=\varphi(c)\langle a, D b\rangle+\varphi(b)\langle c, D a\rangle \quad(a, b, c \in A) .
$$

Let $\lambda \in A^{\prime}$. Then the inner derivation $a d_{\lambda}$ specified by $\lambda$ satisfies

$$
\left\langle b, a d_{\lambda} a\right\rangle=\varphi(b)\langle a, \lambda\rangle-\varphi(a)\langle b, \lambda\rangle \quad(a, b \in A) .
$$

Choose $a_{0} \in A$ with $\varphi\left(a_{0}\right)=1$, and set $\lambda(a)=\left\langle a_{0}, D a\right\rangle(a \in A)$. Then $\lambda$ is a linear functional on $A$, and $|\lambda(a)| \leq\|a\|\|D\|\left\|a_{0}\right\|$, so that $\lambda \in A^{\prime}$ with $\|\lambda\| \leq\|D\|\left\|a_{0}\right\|$. For each $a, b \in A$, it follows from (2.8) and (2.7) that

$$
\begin{aligned}
\left\langle b, a d_{\lambda} a\right\rangle & =\varphi(b)\left\langle a_{0}, D a\right\rangle-\varphi(a)\left\langle a_{0}, D b\right\rangle \\
& =\varphi\left(a_{0}\right)\langle b, D a\rangle=\langle b, D a\rangle .
\end{aligned}
$$

Hence $D=a d_{\lambda}$, and so $A$ is weakly amenable.

The algebra $A$ has no right approximate identity (since $\operatorname{dim} A \geq 2$ ), and so $A$ is not amenable.

It is clear that $R_{A} \subset \operatorname{ker} \varphi=N_{A}=Q_{A}$. Since $\operatorname{ker} \varphi$ is a nil ideal in $A$, we have ker $\varphi \subset R_{A}$. Thus $R_{A}=\operatorname{ker} \varphi$.

Proposition 2.14. Let $A$ be a unital, commutative, weakly amenable Banach algebra, and let $B$ be a Banach algebra such that

$$
b_{1} b_{2}=\varphi\left(b_{1}\right) b_{2} \quad\left(b_{1}, b_{2} \in B\right),
$$

where $\varphi \in \Phi_{B}$. Then $A \widehat{\otimes} B^{\#}$ is weakly amenable.

Proof. Set $\mathfrak{A}=A \widehat{\otimes} B^{\#}$, and let $D: \mathfrak{A} \rightarrow \mathfrak{A}^{\prime}$ be a continuous derivation.

Fix $b_{0}$ in $B$ with $\varphi\left(b_{0}\right)=1$. Then there exists $\lambda \in \mathfrak{A}^{\prime}$ such that

$$
\langle a \otimes b, \lambda\rangle=\left\langle a \otimes b_{0}, D\left(e_{A} \otimes b\right)\right\rangle \quad\left(a \in A, b \in B^{\#}\right) ;
$$

let $a d_{\lambda}: \mathfrak{A} \rightarrow \mathfrak{A}^{\prime}$ be the inner derivation specified by $\lambda$.

Take $a \in A$ and $b, c \in B$. Then

$$
\begin{aligned}
\left\langle a \otimes c, a d_{\lambda}\left(e_{A} \otimes b\right)\right\rangle & =\left\langle a \otimes c,\left(e_{A} \otimes b\right) \cdot \lambda-\lambda \cdot\left(e_{A} \otimes b\right)\right\rangle \\
& =\langle a \otimes c b-a \otimes b c, \lambda\rangle \\
& =\left\langle a \otimes b_{0}, \varphi(c) D\left(e_{A} \otimes b\right)-\varphi(b) D\left(e_{A} \otimes c\right)\right\rangle \\
& =\left\langle a \otimes b_{0},\left(e_{A} \otimes c\right) \cdot D\left(e_{A} \otimes b\right)\right\rangle \\
& =\left\langle a \otimes c, D\left(e_{A} \otimes b\right)\right\rangle,
\end{aligned}
$$

and so $D\left(e_{A} \otimes b\right)=a d_{\lambda}\left(e_{A} \otimes b\right)(b \in B)$. 
We next claim that $D\left(a \otimes e_{B}\right)=a d_{\lambda}\left(a \otimes e_{B}\right)(a \in A)$. For this it is sufficient to show that $D\left(a \otimes e_{B}\right)=0(a \in A)$. In fact $\mathfrak{A}^{\prime}$ is a Banach $A$-bimodule for the products defined by

$$
a \cdot \lambda=\left(a \otimes e_{B}\right) \cdot \lambda, \quad \lambda \cdot a=\lambda \cdot\left(a \otimes e_{B}\right) \quad\left(a \in A, \lambda \in \mathfrak{A}^{\prime}\right) .
$$

Since

$$
\left\langle a_{1} \otimes b,\left(a_{2} \otimes e_{B}\right) \cdot \lambda\right\rangle=\left\langle a_{1} \otimes b, \lambda \cdot\left(a_{2} \otimes e_{B}\right)\right\rangle \quad\left(a_{1}, a_{2} \in A\right),
$$

the dual module $\mathfrak{A}^{\prime}$ is a commutative Banach $A$-bimodule, and the map $a \mapsto D\left(a \otimes e_{B}\right)$ is a continuous derivation into this module. By Proposition 2.2(iv), $D\left(a \otimes e_{B}\right)=0(a \in A)$, as claimed.

Let $a \in A$ and $b \in B$. It follows that

$$
\begin{aligned}
D(a \otimes b) & =D\left(\left(a \otimes e_{B}\right)\left(e_{A} \otimes b\right)\right)=\left(a \otimes e_{B}\right) \cdot D\left(e_{A} \otimes b\right) \\
& =\left(a \otimes e_{B}\right) \cdot D_{\lambda}\left(e_{A} \otimes b\right)=a d_{\lambda}(a \otimes b),
\end{aligned}
$$

and so $D=a d_{\lambda}$ on $\mathfrak{A}$. Thus $\mathfrak{A}$ is weakly amenable.

Munn algebras We present a class of Banach algebras that will play a key role in our later theorems.

Example 2.15. Let $A$ be a unital algebra, let $m, n \in \mathbb{N}$, and let $P$ be a matrix in $\mathbb{M}_{n, m}(A)$. Then $\mathbb{M}_{m, n}(A)$ is an algebra for the product

$$
a \circ b=a P b \quad\left(a, b \in \mathbb{M}_{m, n}(A)\right)
$$

(in the sense of matrix products). This is the

Munn algebra over $A$ with sandwich matrix $P$, and it is denoted by

$$
\mathcal{M}(A, P, m, n)
$$

We write $\mathcal{M}(A, P, n)$ in the special case where $m=n$.

It seems that these algebras were first defined by Munn in [108]. For example, it is proved in [108, Theorem 4.1] that $\mathcal{M}(A, P, m, n)$ is semisimple if and only if $A$ is semisimple, $m=n$, and $P$ is invertible in $\mathbb{M}_{n}(A)$.

Now suppose that $A$ is a unital Banach algebra and that each nonzero element in $P$ has norm 1 . Then $\mathcal{M}(A, P, m, n)$ is also a Banach algebra for the norm of equation (1.4). These Banach algebras are special cases of those defined by Esslamzadeh in [37, Definition 3.1].

We are interested in determining when $\mathcal{M}(A, P, m, n)$ has an identity. We are grateful to John Duncan for pointing out that this does not imply that $m=n$. Indeed, let $C$ be the Banach algebra which is the second Cuntz algebra,

so that $C$ has an identity $e$ and generators $p, q, p^{*}, q^{*}$ such that $p^{*} p=q^{*} q=e, p p^{*}+q q^{*}=e$, and $p^{*} q=q^{*} p=0$, and consider 
the Banach algebra $\mathcal{M}(C, P, 2,1)$, where the sandwich matrix $P$ is the matrix $(p, q)$. Then it is easy to check that the matrix which is the transpose of $\left(p^{*}, q^{*}\right)$ is the identity of $\mathcal{M}(C, P, 2,1)$. (For further properties of the Cuntz algebra $C$, see [25].)

Suppose that each non-zero element of $P$ is invertible in $A$ and that $\mathcal{M}(A, P, m, n)$ has an identity. It is stated in the proof of $[\mathbf{3 7}$, Theorem 4.1(i)] that this implies that $m=n$ and that $P$ is invertible in $\mathbb{M}_{n}(A)$. However, we are not able to follow the proof of this claim; indeed in the algebraic reduction in the proof of [37, Lemma 3.6] we obtain from $P$ an element in $\mathbb{M}_{n, m}(A)$ that is the difference of two elements in the matrix $P$, and we may suppose that this element is non-zero, but we see no reason for it to be invertible in $A$. Our Proposition 2.16, below, is a small variant of these claimed results; we are grateful to John Duncan for telling this to us. See also [17, Lemma 5.18].

Proposition 2.16. Let $A$ be a unital algebra with a character, and consider the Munn algebra

$\mathfrak{A}=\mathcal{M}(A, P, m, n)$. Suppose that $\mathfrak{A}$ has an identity. Then $m=n$, $P$ is an invertible matrix in $\mathbb{M}_{n}(A)$, and

$$
\theta: X \mapsto P^{-1} X, \mathbb{M}_{n}(A) \rightarrow \mathcal{M}(A, P, n),
$$

is an isomorphism.

Proof. The specified character on $A$ is $\varphi$; for $X=\left(x_{i j}\right) \in \mathbb{M}_{r, s}(A)$, set $\varphi(X)=\left(\varphi\left(x_{i j}\right)\right) \in \mathbb{M}_{r, s}$.

Since $\mathfrak{A}$ has an identity, there is an element $Q \in \mathbb{M}_{m, n}(A)$ with

$$
X P Q=Q P X=X \quad\left(X \in \mathbb{M}_{m, n}(A)\right) .
$$

Thus

$$
M \varphi(P) \varphi(Q)=\varphi(Q) \varphi(P) M=M \quad\left(M \in \mathbb{M}_{m, n}\right) .
$$

It follows that $\varphi(P) \varphi(Q)=I_{n}$ and that $\varphi(Q) \varphi(P)=I_{m}$. This is only possible in the special case where $m=n$. Further, it now follows from (2.9) that $P Q=Q P$ is the identity of $\mathbb{M}_{n}(A)$, and so $Q=P^{-1}$.

Clearly, the map $\theta$ is an isomorphism.

Let $A$ be a unital Banach algebra with a character, and again set $\mathfrak{A}=\mathcal{M}(A, P, m, n)$. Suppose that $m=n$ and that $P$ is an invertible matrix in $\mathbb{M}_{n}(A)$, with inverse $Q$. Then the above map $\theta$ is a topological isomorphism; we shall need the norm of this isomorphism. Set

$$
v(P)=\max \left\{\sum_{r=1}^{n}\left\|Q_{r i}\right\|: i \in \mathbb{N}_{n}\right\} .
$$


Then clearly

$$
\begin{aligned}
\|\theta\| & =\max \left\{\left\|Q\left(E_{i j}\right)\right\|: i, j \in \mathbb{N}_{n}\right\} \\
& =\max \left\{\sum_{r=1}^{n}\left\|Q_{r i}\right\|: i \in \mathbb{N}_{n}\right\}=v(P) .
\end{aligned}
$$

Suppose further that $A$ is $C$-amenable. Then the Banach algebra $\mathfrak{A}$ is $C n v(P)^{2}$-amenable; this follows from Proposition 2.5 and Theorem 2.7 (iv).

Second duals of Banach algebras Again let $A$ be a Banach algebra. As above, $A^{\prime \prime}$ is a Banach $A$-bimodule for maps $(a, \Phi) \mapsto a \cdot \Phi$ and $(a, \Phi) \mapsto \Phi \cdot a$ from $A \times A^{\prime \prime}$ to $A^{\prime \prime}$ that extend the product map $m_{A}$. There are two products on the second dual space $A^{\prime \prime}$ of $A$ which extend these module maps; these products are denoted by $\square$ and $\diamond$, and are called the first and second Arens products on $A$;

the original definitions of the two products were given in $[\mathbf{2}],[\mathbf{3}]$. An early account of second duals of Banach algebras and Arens products is given in $[31]$; a comprehensive recent survey is $[46]$.

We recall briefly the definitions of $\square$ and $\diamond$; a fuller discussion of these products is given in $[\mathbf{2 1}]$.

First, for $\lambda \in A^{\prime}$, we have

$$
\langle b, a \cdot \lambda\rangle=\langle b a, \lambda\rangle, \quad\langle b, \lambda \cdot a\rangle=\langle a b, \lambda\rangle \quad(a, b \in A) .
$$

Now, for $\lambda \in A^{\prime}$ and $\Phi \in A^{\prime \prime}$, define $\lambda \cdot \Phi$ and $\Phi \cdot \lambda$ in $A^{\prime}$ by

$$
\langle a, \lambda \cdot \Phi\rangle=\langle\Phi, a \cdot \lambda\rangle, \quad\langle a, \Phi \cdot \lambda\rangle=\langle\Phi, \lambda \cdot a\rangle \quad(a \in A) .
$$

Finally, for $\Phi, \Psi \in A^{\prime \prime}$, define

$$
\langle\Phi \square \Psi, \lambda\rangle=\langle\Phi, \Psi \cdot \lambda\rangle, \quad\langle\Phi \diamond \Psi, \lambda\rangle=\langle\Psi, \lambda \cdot \Phi\rangle \quad\left(\lambda \in A^{\prime}\right) .
$$

Suppose that $\Phi=\lim _{\alpha} a_{\alpha}$ and $\Psi=\lim _{\beta} b_{\beta}$ for nets $\left(a_{\alpha}\right)$ and $\left(b_{\beta}\right)$ in A. Then

$$
\Phi \square \Psi=\lim _{\alpha} \lim _{\beta} a_{\alpha} b_{\beta}, \quad \Phi \diamond \Psi=\lim _{\beta} \lim _{\alpha} a_{\alpha} b_{\beta},
$$

where all limits are taken in the $\sigma\left(A^{\prime \prime}, A^{\prime}\right)$-topology on $A^{\prime \prime}$.

TheOREM 2.17. Let $A$ be a Banach algebra. Then both $\left(A^{\prime \prime}, \square\right)$ and $\left(A^{\prime \prime}, \diamond\right)$ are Banach algebras containing $A$ as a closed subalgebra.

In the case where $A$ is commutative, $\lambda \cdot \Phi=\Phi \cdot \lambda\left(\lambda \in A^{\prime}, \Phi \in A^{\prime \prime}\right)$, and $\Phi \square \Psi=\Psi \diamond \Phi\left(\Phi, \Psi \in A^{\prime \prime}\right)$, so that $\left(A^{\prime \prime}, \diamond\right)=\left(A^{\prime \prime}, \square\right)^{\text {op }}$.

We note that $R_{\Phi}: \Psi \mapsto \Psi \square \Phi$ is continuous on $\left(A^{\prime \prime}, \sigma\left(A^{\prime \prime}, A\right)\right)$ for each $\Phi \in A^{\prime \prime}$, and that $L_{a}: \Psi \mapsto a \square \Psi$ is continuous on $\left(A^{\prime \prime}, \sigma\left(A^{\prime \prime}, A\right)\right)$ for each $a \in A$. 
Let $A$ be a Banach algebra without an identity. Then it is clear that we can identify $\left(A^{\# \prime \prime}, \square\right)$ with $\left.\left(A^{\prime \prime}, \square\right)\right)^{\#}$.

The following result is [50, Proposition 1.3].

Proposition 2.18. Let $A$ be a Banach algebra.

(i) Let I be a right ideal in $\left(A^{\prime \prime}, \square\right)$. Then $\bar{I}^{\sigma}$ is also a right ideal.

(ii) Let I be a left ideal in $\left(A^{\prime \prime}, \square\right)$. Then $\bar{I}^{\sigma}$ is also a left ideal.

The following result is part of [19, Proposition 2.6.25].

Proposition 2.19. Let $A$ be a Banach algebra. Then $A$ is a full subalgebra of $\left(A^{\prime \prime}, \square\right)$ and $R_{\left(A^{\prime \prime}, \square\right)} \cap A \subset R_{A}$. In the case where $A$ is commutative, $R_{\left(A^{\prime \prime}, \square\right)} \cap A=R_{A}$.

Let $A$ and $B$ be Banach algebras, and let $\theta: A \rightarrow B$ be a continuous homomorphism. Then $\theta^{\prime \prime}:\left(A^{\prime \prime}, \square\right) \rightarrow\left(B^{\prime \prime}, \square\right)$ is a continuous homomorphism. Let $A$ be a closed subalgebra of $B$. Then we regard $\left(A^{\prime \prime}, \square\right)$ as a closed subalgebra of $\left(B^{\prime \prime}, \square\right)$. Let $\varphi \in \Phi_{A}$. Then

$$
\varphi^{\prime \prime}: \Psi \mapsto\langle\Psi, \varphi\rangle, \quad\left(A^{\prime \prime}, \square\right) \rightarrow \mathbb{C},
$$

is a character on $A^{\prime \prime}$ which extends $\varphi$.

Details of the following result are given in [38, Lemma 3.2].

Proposition 2.20. Let $A$ be a Banach algebra, and let $n \in \mathbb{N}$. Then $\left.\mathbb{M}_{n}\left(A^{\prime \prime}, \square\right)=\left(\mathbb{M}_{n}(A)^{\prime \prime}, \square\right)\right)$.

Proposition 2.21. Let $A$ be a Banach algebra, and let $B$ be a closed subalgebra of $\left(A^{\prime \prime}, \square\right)$ with $A \subset B$. Then:

(i) $B$ is a full subalgebra of $\left(A^{\prime \prime}, \square\right)$;

(ii) the restriction map $R: \varphi \mapsto \varphi \mid B, \Phi_{\left(A^{\prime \prime}, \square\right)} \rightarrow \Phi_{B}$, is a continuous surjection.

Proof. (i) Let $b \in B$, and suppose that $b$ is quasi-invertible in $\left(A^{\prime \prime}, \square\right)$. Then $b$ is quasi-invertible in $\left(B^{\prime \prime}, \square\right)$. By Proposition $2.19, b$ is quasi-invertible in $B$. Thus $B$ is a full subalgebra of $\left(A^{\prime \prime}, \square\right)$.

(ii) The map $R$ is clearly continuous. Suppose that $\psi \in \Phi_{B}$. Then $\varphi:=\psi^{\prime \prime} \mid A^{\prime \prime} \in \Phi_{\left(A^{\prime \prime}, \square\right)}$, and $R(\varphi)=\psi$, so that $R$ is a surjection.

The continuous bilinear map $M:(a, b) \mapsto a \otimes b, A \times A \rightarrow A \widehat{\otimes} A$, has an extension to a continuous bilinear map $\widetilde{M}: A^{\prime \prime} \times A^{\prime \prime} \rightarrow(A \widehat{\otimes} A)^{\prime \prime}$ 
with $\|\widetilde{M}\|=1$; see [19, Definition A.3.51]. Indeed, as in equation (A.3.8) of [19], we have

$$
\widetilde{M}(\Phi, \Psi)=\lim _{\alpha} \lim _{\beta} M\left(a_{\alpha}, b_{\beta}\right)
$$

when $\lim _{\alpha} a_{\alpha}=\Phi$ and $\lim _{\beta} b_{\beta}=\Psi$, where we take limits in the weak-* topology on $(A \widehat{\otimes} A)^{\prime \prime}$. It follows that

$$
\widetilde{M}(a \cdot \Phi, \Psi \cdot b)=a \cdot \widetilde{M}(\Phi, \Psi) \cdot b \quad\left(a, b \in A, \Phi, \Psi \in A^{\prime \prime}\right) .
$$

Thus there is an $A$-bimodule homomorphism

$$
\kappa: A^{\prime \prime} \widehat{\otimes} A^{\prime \prime} \rightarrow(A \widehat{\otimes} A)^{\prime \prime}
$$

such that $\kappa$ is the identity map on $A \widehat{\otimes} A$ and $\|\kappa\|=1$. This map apparently first occurs in [64], and the above remark is explicitly given in [54, Lemma 1.7].

Proposition 2.22. Let $A$ be a Banach algebra, and suppose that $\left(A^{\prime \prime}, \square\right)$ is amenable. Then $A$ is amenable, and $A M(A) \leq A M\left(A^{\prime \prime}\right)$.

Proof. The fact that $A$ is amenable is given in $[\mathbf{1 9}$, Proposition 2.8.59(ii)]; the result is due to Gourdeau [59].

In fact, set $C=A M\left(A^{\prime \prime}\right)$, and let $\left(U_{\alpha}\right)$ be a bounded approximate diagonal for $A^{\prime \prime}$ with $\sup _{\alpha}\left\|U_{\alpha}\right\|_{\pi}=C$. Set $u_{\alpha}=\kappa\left(U_{\alpha}\right)$ for each $\alpha$. Then $\left(u_{\alpha}\right)$ is a bounded net in $(A \widehat{\otimes} A)_{[C]}^{\prime \prime}$; a weak-* accumulation point of $\left(u_{\alpha}\right)$ in $(A \widehat{\otimes} A)_{[C]}^{\prime \prime}$ is a virtual diagonal for $A$, and so $A$ is $C$-amenable. Thus $A M(A) \leq A M\left(A^{\prime \prime}\right)$.

Let $A$ be a Banach algebra. It is not known whether or not the weak amenability of $\left(A^{\prime \prime}, \square\right)$ implies that of $A$. For some partial results, see $[27],[49]$, and $[36]$.

The following definition is taken from [124, Definition 1.1] and [21, Definition 1.6].

Definition 2.23. Let $A$ be a Banach algebra. Then $A$ is a dual Banach algebra

if there is a closed submodule $E$ of $A^{\prime}$ such that $E^{\prime}=A$.

In fact, let $E$ be a Banach space such that $E^{\prime}=A$. Then $A$ is a dual Banach algebra with respect to $E$ if and only if the product in $A$ is separately continuous in the $\sigma(A, E)$ topology. In this case, $E^{\circ}$ is a $\sigma\left(A^{\prime \prime}, A^{\prime}\right)$-closed (and hence $\|\cdot\|$-closed) ideal in $\left(A^{\prime \prime}, \square\right)$ and $A^{\prime \prime}=A \ltimes E^{\circ}$ as a semidirect product [21, Theorem 2.15]. In particular, $E^{\circ}$ is a closed, complemented ideal in $A^{\prime \prime}$. We shall show in Example 4.11 that it may be that there are two Banach spaces $E$ and $F$ with 
$E^{\prime}=F^{\prime}=A$ such that $A$ is a dual Banach algebra with respect to $F$, but not with respect to $E$.

For a recent account of dual Banach algebras, see [28]. It is shown in $[49]$ that $A$ is weakly amenable whenever $A$ is a dual Banach algebra and $\left(A^{\prime \prime}, \square\right)$ is weakly amenable.

\section{Topological centres}

We first recall the standard definition of a much-studied concept, that of the topological centres of the second dual of a Banach algebra. For details of all the following remarks, and many examples for which $\mathfrak{Z}_{t}^{(\ell)}\left(A^{\prime \prime}\right)$ and $\mathfrak{Z}_{t}^{(r)}\left(A^{\prime \prime}\right)$ of $A^{\prime \prime}$ are determined, see the memoir [21]; however, we have introduced for convenience a small variation in the terminology from that source.

Definition 2.24. Let $A$ be a Banach algebra. The left and right topological centres, $\mathfrak{Z}_{t}^{(\ell)}\left(A^{\prime \prime}\right)$ and $\mathfrak{Z}_{t}^{(r)}\left(A^{\prime \prime}\right)$, of $A^{\prime \prime}$ are

$$
\begin{aligned}
& \mathfrak{Z}_{t}^{(\ell)}\left(A^{\prime \prime}\right)=\left\{\Phi \in A^{\prime \prime}: \Phi \square \Psi=\Phi \diamond \Psi\left(\Psi \in A^{\prime \prime}\right)\right\}, \\
& \mathfrak{Z}_{t}^{(r)}\left(A^{\prime \prime}\right)=\left\{\Phi \in A^{\prime \prime}: \Psi \square \Phi=\Psi \diamond \Phi\left(\Psi \in A^{\prime \prime}\right)\right\},
\end{aligned}
$$

respectively. The algebra $A$ is Arens regular if

$$
\mathfrak{Z}_{t}^{(\ell)}\left(A^{\prime \prime}\right)=\mathfrak{Z}_{t}^{(r)}\left(A^{\prime \prime}\right)=A^{\prime \prime}
$$

and $A$ is left strongly Arens irregular if $\mathfrak{Z}_{t}^{(\ell)}\left(A^{\prime \prime}\right)=A$, right strongly Arens irregular if $\mathfrak{Z}_{t}^{(r)}\left(A^{\prime \prime}\right)=A$, and strongly Arens irregular if it is both left and right strongly Arens irregular.

It is clear that $\mathfrak{Z}_{t}^{(\ell)}\left(A^{\prime \prime}\right)$ and $\mathfrak{Z}_{t}^{(r)}\left(A^{\prime \prime}\right)$ are both closed linear subspaces of $A^{\prime \prime}$. In fact, each is a subalgebra of both of $\left(A^{\prime \prime}, \square\right)$ and of $\left(A^{\prime \prime}, \diamond\right)$. Indeed, for example, let $\Phi_{1}, \Phi_{2} \in \mathfrak{Z}_{t}^{(\ell)}\left(A^{\prime \prime}\right)$. Then, for each $\Psi \in A^{\prime \prime}$, we have

$$
\left(\Phi_{1} \square \Phi_{2}\right) \square \Psi=\Phi_{1} \square\left(\Phi_{2} \square \Psi\right)=\Phi_{1} \diamond\left(\Phi_{2} \square \Psi\right)
$$

and

$$
\left(\Phi_{1} \square \Phi_{2}\right) \diamond \Psi=\left(\Phi_{1} \diamond \Phi_{2}\right) \diamond \Psi=\Phi_{1} \diamond\left(\Phi_{2} \diamond \Psi\right),
$$

and so $\Phi_{1} \square \Phi_{2} \in \mathfrak{Z}_{t}^{(\ell)}\left(A^{\prime \prime}\right)$.

Thus $A$ is Arens regular if and only if the two products $\square$ and $\diamond$ coincide on $A^{\prime \prime}$. In the case where $A$ is a commutative Banach algebra, $\mathfrak{Z}_{t}^{(\ell)}\left(A^{\prime \prime}\right)=\mathfrak{Z}_{t}^{(r)}\left(A^{\prime \prime}\right)=\mathfrak{Z}\left(A^{\prime \prime}, \square\right)$, and $A$ is Arens regular if and only if $\left(A^{\prime \prime}, \square\right)$ is commutative. For example, each $C^{*}$-algebra is Arens regular and each group algebra of the form $L^{1}(G)$ is strongly Arens irregular [95]. Some history of the calculations of the topological centres of $A^{\prime \prime}$ 
when $A=L^{1}(G)$ for a locally compact group $G$ and $A=\ell^{1}(S)$ for a semigroup $S$ is given in Chapters 8 and 12, respectively.

It is shown in [49, Corollary 1.2] that $A$ is amenable

whenever $\mathfrak{Z}_{t}^{(\ell)}\left(A^{\prime \prime}\right)$ is amenable.

Let $A$ be a Banach algebra, and let $\Phi \in A^{\prime \prime}$. Then the following are equivalent: (a) $\Phi \in \mathfrak{Z}_{t}^{(\ell)}\left(A^{\prime \prime}\right)$; (b) $L_{\Phi}: \Psi \mapsto \Phi \square \Psi$ is continuous on $\left(A^{\prime \prime}, \sigma\left(A^{\prime \prime}, A^{\prime}\right)\right)$; (c) $\Phi \cdot a_{\alpha} \rightarrow \Phi \square \Psi$ whenever $\left(a_{\alpha}\right)$ is a net in $A$ with $\lim _{\alpha} a_{\alpha}=\Psi$. The map $L_{\Phi}$ is continuous on $\left(A^{\prime \prime}, \sigma\left(A^{\prime \prime}, A^{\prime}\right)\right)$ for each $\Phi \in A^{\prime \prime}$ if and only if $A$ is Arens regular. It is easy to see that

$$
\mathfrak{Z}\left(A^{\prime \prime}, \square\right) \subset \mathfrak{Z}_{t}^{(\ell)}\left(A^{\prime \prime}\right) \cap \mathfrak{Z}_{t}^{(r)}\left(A^{\prime \prime}\right) .
$$

Proposition 2.25. Let $A$ be a Banach algebra with a closed ideal I. Suppose that $A / I$ and $I$ are strongly Arens irregular. Then $A$ is strongly Arens irregular.

Proof. Set $B=A / I$; the quotient map is $q: A \rightarrow B$.

Let $\Phi \in \mathfrak{Z}_{t}^{(\ell)}\left(A^{\prime \prime}\right)$, so that $\Phi \square \Psi=\Phi \diamond \Psi\left(\Psi \in A^{\prime \prime}\right)$. We have

$$
q^{\prime \prime}(\Phi) \square q^{\prime \prime}(\Psi)=q^{\prime \prime}(\Phi) \diamond q^{\prime \prime}(\Psi) \quad\left(\Psi \in A^{\prime \prime}\right),
$$

and so $q^{\prime \prime}(\Phi) \in \mathfrak{Z}_{t}^{(\ell)}\left(B^{\prime \prime}\right)$. Since $B$ is strongly Arens irregular, we see that $q^{\prime \prime}(\Phi) \in B$, say $q^{\prime \prime}(\Phi)=q\left(a_{0}\right)$, where $a_{0} \in A$. By replacing $\Phi$ by $\Phi-a_{0}$, we may suppose that $q^{\prime \prime}(\Phi)=0$, and so $\Phi \in \mathfrak{Z}_{t}^{(\ell)}\left(I^{\prime \prime}\right)$. Since $I$ is strongly Arens irregular, $\Phi \in I$. Hence $A$ is left strongly Arens irregular. Similarly, $A$ is right strongly Arens irregular.

Let $A$ be a Banach algebra, and let $\mathcal{M}(A, P, m, n)$ be a Munn algebra. It is clear [38, Proposition 4.1] that

$$
\mathcal{M}\left(\mathfrak{Z}_{t}^{(\ell)}\left(A^{\prime \prime}\right), P, m, n\right) \subset \mathfrak{Z}_{t}^{(\ell)}\left(\left(\mathcal{M}(A, P, m, n)^{\prime \prime}\right) ;\right.
$$

equality holds in the case where $m=n$ and $P$ is invertible in $\mathbb{M}_{n}(A)$, and so in this case $\mathcal{M}(A, P, n)$ is strongly Arens irregular if and only if $A$ is strongly Arens irregular.

The following result is an abstract version of part of the proof of [54, Theorem 1.3].

Proposition 2.26. Let $A$ be an infinite-dimensional, dual Banach algebra for a closed submodule $E$ of $A^{\prime}$ with $E^{\prime}=A$. Suppose that $\left(A^{\prime \prime}, \square\right)$ is amenable. Then $\left(E^{\circ}, \square\right)$ has an identity, and $A$ is not left strongly Arens irregular.

Proof. By Proposition 2.2(vii), the ideal $\left(E^{\circ}, \square\right)$ has a bounded approximate identity, say $\left(\Phi_{\alpha}\right)$. By passing to a subnet, we may suppose that $\Phi_{\alpha} \rightarrow \Phi_{0}$ in $\left(A^{\prime \prime}, \sigma\left(A^{\prime \prime}, A^{\prime}\right)\right)$, and then $\Phi_{0} \in E^{\circ}$. 
Let $\Phi \in E^{\circ}$. Then we have $\lim _{\alpha} \Phi_{\alpha} \square \Phi=\Phi$ in $\left(A^{\prime \prime},\|\cdot\|\right)$ and $\lim _{\alpha} \Phi_{\alpha} \square \Phi=\Phi_{0} \square \Phi$ in $\left(A^{\prime \prime}, \sigma\left(A^{\prime \prime}, A^{\prime}\right)\right)$, and so $\Phi_{0} \square \Phi=\Phi$. Also,

$$
\Phi \square \Phi_{0}=\lim _{\alpha}\left(\Phi_{\square} \Phi_{0}\right) \square \Phi_{\alpha}=\lim _{\alpha} \Phi \square \Phi_{\alpha}=\Phi,
$$

taking limits in $\left(A^{\prime \prime},\|\cdot\|\right)$. Hence $\Phi_{0}$ is the identity for $E^{\circ}$.

Let $a \in A$. Then $a \cdot \Phi_{0}, \Phi_{0} \cdot a \in E^{\circ}$, and so

$$
\Phi_{0} \cdot a=\left(\Phi_{0} \cdot a\right) \cdot \Phi_{0}=\Phi_{0} \cdot\left(a \cdot \Phi_{0}\right)=a \cdot \Phi_{0} .
$$

It follows that $\Phi_{0} \in \mathfrak{Z}\left(A^{\prime \prime}, \square\right) \subset \mathfrak{Z}_{t}^{(\ell)}\left(A^{\prime \prime}\right)$, and so $\Phi_{0} \neq 0$. Thus $\Phi_{0} \notin A$, and hence $A$ is not left strongly Arens irregular.

Definition 2.27. Let $A$ be a Banach algebra. A linear functional $\lambda \in A^{\prime}$ is [weakly] almost periodic if the map

$$
a \mapsto a \cdot \lambda, \quad A \rightarrow A^{\prime},
$$

is [weakly] compact. The spaces of almost periodic and weakly almost periodic functionals on $A$ are denoted by $A P(A)$ and $W A P(A)$, respectively.

Both $A P(A)$ and $W A P(A)$ are $\|\cdot\|$-closed $A$-submodules of $A^{\prime}$, and $A P(A) \subset W A P(A)$. In fact, as in [119],

(2.15) $W A P(A)=\left\{\lambda \in A^{\prime}:\langle\Phi \square \Psi, \lambda\rangle=\langle\Phi \diamond \Psi, \lambda\rangle\left(\Phi, \Psi \in A^{\prime \prime}\right)\right\}$,

and so $A$ is Arens regular if and only if $W A P(A)=A^{\prime}$.

The following well-known characterization of Arens regularity is taken from [19, Theorem 2.6.17].

Theorem 2.28. Let $A$ be a Banach algebra. Then the following conditions on $A$ are equivalent.

(a) $A$ is

Arens regular;

(b) for each $\Phi \in A^{\prime \prime}$, the map $L_{\Phi}$ is continuous on $\left(A^{\prime \prime}, \sigma\left(A^{\prime \prime}, A^{\prime}\right)\right)$;

(c) for each $\lambda \in A^{\prime}$ and bounded sequences $\left(a_{m}\right)$ and $\left(b_{n}\right)$ in $A$,

$$
\lim _{m} \lim _{n}\left\langle a_{m} b_{n}, \lambda\right\rangle=\lim _{m} \lim _{n}\left\langle a_{m} b_{n}, \lambda\right\rangle
$$

whenever both repeated limits exist;

(d) $W A P(A)=A^{\prime}$.

Corollary 2.29. Let $A$ be an Arens regular Banach algebra. Then closed subalgebras of $A$ and quotients of $A$ by closed ideals are also Arens regular. 
For example, let $A=\left(\ell^{1}, \cdot\right)$. Then $A$ is Arens regular $[\mathbf{1 9}$, Example 2.6.22(iii)].

Introverted subspaces We now define introverted subspaces of $A^{\prime}$, where $A$ is a Banach algebra. A form of this definition goes back to Day $[29]$.

Definition 2.30. Let $A$ be a Banach algebra, and let $X$ be a $\|\cdot\|$ closed, A-submodule of $A^{\prime}$. Then $X$ is left-introverted if

$$
\Phi \cdot \lambda \in X \quad\left(\lambda \in X, \Phi \in A^{\prime \prime}\right),
$$

and $X$ is right-introverted if

$$
\lambda \cdot \Phi \in X \quad\left(\lambda \in X, \Phi \in A^{\prime \prime}\right) .
$$

Further, the submodule $X$ is introverted if it is both left-introverted and right-introverted in $A^{\prime}$.

For example, $A^{\prime}$ and $\overline{A^{\prime} A}$ are both left-introverted subspaces of $A^{\prime}$. It is shown in [21, Proposition 5.7] that each $\|\cdot\|$-closed, $A$-submodule $X$ of $A^{\prime}$ for which $X \subset W A P(A)$ is introverted. In particular, $A P(A)$ and $W A P(A)$ are each introverted subspaces of $A^{\prime}$. It follows from (2.15) that the products $\square$ and $\diamond$ on $X^{\prime}$ coincide whenever $X$ is a faithful, introverted subspace of $A^{\prime}$ with $X \subset W A P(A)$.

Let $A$ be a Banach algebra, and let $X$ be a faithful, left-introverted subspace of $A^{\prime}$. Then $X^{\circ}$ is a closed ideal in $\left(A^{\prime \prime}, \square\right)$, and so we can regard $\left(X^{\prime}, \square\right)=\left(A^{\prime \prime}, \square\right) / X^{\circ}$ as a quotient Banach algebra, with quotient map denoted by $\pi_{X}$. Similarly, $\left(X^{\prime}, \diamond\right)$ is a quotient Banach algebra of $\left(A^{\prime \prime}, \diamond\right)$ in the case where $X$ is right-introverted. We regard $A$ as a closed subalgebra of $\left(X^{\prime}, \square\right)$ and $\left(X^{\prime}, \diamond\right)$, as appropriate. Let $X$ be an introverted subspace of $A^{\prime}$. Then the products $\square$ and $\diamond$ in $X^{\prime}$ are specified by

$$
\langle\Phi \square \Psi, \lambda\rangle=\langle\Phi, \Psi \cdot \lambda\rangle, \quad\langle\Phi \diamond \Psi, \lambda\rangle=\langle\Psi, \lambda \cdot \Phi\rangle \quad\left(\lambda \in X^{\prime}\right)
$$

for $\Phi, \Psi \in A^{\prime \prime}$.

Suppose, further, that $A$ is a dual Banach algebra with respect to $E \subset A^{\prime}$, and that $E \subset X$. Then we have

$$
X^{\prime}=A \ltimes\left(E^{\circ} / X^{\circ}\right)
$$

as a semi-direct product. See [21, Chapter 6] for details of the above and for several further examples of introverted subspaces; we shall return to this notion in Chapters 8 and 12 . 
Definition 2.31. Let $A$ be a Banach algebra, and let $X$ be a leftintroverted subspace of $A^{\prime}$. Then the topological centre of $X^{\prime}$ is

$\mathfrak{Z}_{t}\left(X^{\prime}\right)=\left\{\Phi \in X^{\prime}: L_{\Phi}\right.$ is continuous on $\left.\left(X^{\prime}, \sigma\left(X^{\prime}, X\right)\right)\right\}$.

We have $A \subset \mathfrak{Z}_{t}\left(X^{\prime}\right) \subset X^{\prime}$. In the case where $X=A^{\prime}$, the set $\mathfrak{Z}_{t}\left(X^{\prime}\right)$ coincides with the previously defined left topological centre $\mathfrak{Z}_{t}^{(\ell)}\left(A^{\prime \prime}\right)$. Suppose that $A$ is Arens regular. Then $\mathfrak{Z}_{t}\left(X^{\prime}\right)=X^{\prime}$ for each left-introverted subspace $X$ of $A^{\prime}$. In the case where $X$ is introverted, we have

$$
\mathfrak{Z}_{t}\left(X^{\prime}\right)=\left\{\Phi \in X^{\prime}: \Phi \square \Psi=\Phi \diamond \Psi\left(\Psi \in X^{\prime}\right)\right\} .
$$

The notion of the topological centre $\mathfrak{Z}_{t}\left(X^{\prime}\right)$ in the above sense was introduced in [82]; see [99] for the case where $X=\overline{A^{\prime} A}$. 



\section{CHAPTER 3}

\section{Semigroups}

In this chapter, we shall recall certain basic algebraic properties of semigroups, and then shall move on to the consideration of some semigroups which are also topological spaces, in particular of compact, right topological semigroups. We shall include for background information some known facts that are not actually to be used. The chapter will conclude with a collection of examples.

For an introductory account of semigroup theory, see [81], for example. The standard work on this subject is $[\mathbf{1 7}]$.

Basic definitions A semigroup is a non-empty set with an associative product, usually denoted by juxtaposition; however, the semigroup product in $\mathbb{Z}$ (and also $\mathbb{Z}^{+}$and $\mathbb{N}$ ) is always addition, denoted by + , unless otherwise stated. Of course, each group

is a semigroup.

Let $S$ be a semigroup. The product map is

$$
m_{S}:(s, t) \mapsto s t, \quad S \times S \rightarrow S .
$$

In the case where $S$ is unital, the identity of $S$ is denoted by $e_{S}$. Suppose that $S$ is non-unital. Then the semigroup formed by adjoining an identity to $S$ is denoted by $S^{\#}$; even in this case we sometimes write $e_{S} s$ for $s$ when $s \in S$. The opposite semigroup to $S$ is the same set $S$ with the opposite product $\cdot$, defined by setting $s \cdot t=t s(s, t \in S)$; the opposite semigroup is denoted by $S^{\text {op }}$. The semigroup $S$ is abelian if $s t=t s(s, t \in S)$. A non-empty subset $T$ of $S$ is a subsemigroup if $T$ is a semigroup for the product in $S$. For $s \in S$, we set

$$
\langle s\rangle=\left\{s^{n}: n \in \mathbb{N}\right\},
$$

the semigroup generated by $s$; the subsemigroup of $S$ generated by a subset $T$ is $\langle T\rangle$; a semigroup $S$ is finitely generated if there exists $T \in \mathcal{P}_{f}(S)$ with $\langle T\rangle=S$, and $S$ is infinitely generated if it is not finitely generated.

There is a notion related to that of $\langle T\rangle$ when $T$ is replaced by a (finite or infinite) sequence $\left(s_{n}\right)$ in $S$. This is the set of finite products of $\left(s_{n}\right)$, defined by 


$$
F P\left\langle\left(s_{n}\right)\right\rangle=\bigcup_{k \in \mathbb{N}}\left\{s_{n_{1}} \cdots s_{n_{k}}: n_{1}, \ldots, n_{k} \in \mathbb{N}, n_{1}<n_{2}<\cdots<n_{k}\right\} ;
$$

in the case where $S=(\mathbb{N},+)$ or $S=(\mathbb{Z},+)$ and $\left(s_{n}\right)$ is a sequence in $S$, we refer to the finite sums of $\left(s_{n}\right)$, defined by

$$
F S\left\langle\left(s_{n}\right)\right\rangle=\bigcup_{k \in \mathbb{N}}\left\{s_{n_{1}}+\cdots+s_{n_{k}}: n_{1}, \ldots, n_{k} \in \mathbb{N}, n_{1}<n_{2}<\cdots<n_{k}\right\} .
$$

The sequence $\left(s_{n}\right)$ has distinct finite products if the elements $s_{n_{1}} \cdots s_{n_{k}}$ in $F P\left\langle\left(s_{n}\right)\right\rangle$ are uniquely determined by the strictly increasing sequence $\left(n_{1}, \ldots, n_{k}\right)$ in $\mathbb{N}$;

see [78, Definitions 5.1 and 6.26].

Let $S$ and $T$ be two semigroups. A morphism from $S$ to $T$ is a map $\theta: S \rightarrow T$ such that $\theta\left(s_{1} s_{2}\right)=\theta\left(s_{1}\right) \theta\left(s_{2}\right)\left(s_{1}, s_{2} \in S\right)$; an epimorphism is a surjective morphism, and an isomorphism is a bijective morphism. The semigroups $S$ and $T$ are isomorphic, written $S \cong T$, if there is an isomorphism from $S$ onto $T$.

DeFinition 3.1. Let $S$ be a semigroup, and let $o \in S$ be such that

$$
s o=o s=o \quad(s \in S) .
$$

Then o is a zero for the semigroup $S$.

Let $S$ be a semigroup, and let $o$ be an element not in $S$. Set $T=S \cup\{o\}$, and define $s o=o s=o(s \in S)$ and $o^{2}=o$. Then $T$ is semigroup containing $S$ as a subsemigroup; we say that $T$ is formed by adjoining a zero to $S$, and write $T=S^{\circ}$. Suppose that $S$ has an identity $e_{S}$. Then $e_{S}$ is also the identity of $S^{\circ}$.

The following elementary semigroup $D$ will be very useful later.

ExAmple 3.2. Let $D$ be the two-element set $\{e, o\}$ with products $e e=e$ and $e o=o e=o^{2}=o$. Then $D$ is a semigroup with identity $e$ and zero $o$. Let $S$ be any semigroup, and let $S^{o}$ be the semigroup formed by adjoining a zero to $S$. Then the map $\theta: S^{o} \rightarrow D$ specified by setting $\theta(s)=e(s \in S)$ and $\theta(o)=o$ is an epimorphism.

DeFinition 3.3. Let $S$ be a semigroup. Then $S$ is regular

if, for each $s$ in $S$, there exists $t \in S$ with sts $=s$. An element $p \in S$ is an idempotent if $p^{2}=p$; the set of idempotents of $S$ is denoted by $E(S)$. 
Of course, a group $G$ is a regular semigroup such that $E(G)=\left\{e_{G}\right\}$.

Let $S$ be a regular semigroup. Then, for each $s \in S$, there exists $u \in S$ with $s u s=s$ and $u s u=u$. Indeed, take $t \in S$ with $s t s=s$, and set $u=t s t$; it is immediately checked that $s u s=s$ and $u s u=u$. In this case, $u s, s u \in E(S)$, and so $E(S) \neq \emptyset$. It follows that

$$
S=\bigcup\{p S q: p, q \in E(S)\} .
$$

Let $\leq$ be a transitive and reflexive relation on a set $S$. Then $\leq$ is a quasi-order; it is a partial order if, further, it is anti-symmetric. Let $\leq$ be a quasi-order on a set $S$. An element $s_{0}$ is a maximum if $s \leq s_{0} \quad(s \in S)$ and maximal if $s \leq s_{0}$ whenever $s \in S$ and $s_{0} \leq s$; minimum and minimal elements are defined similarly.

There are several orderings on the subset $E(S)$ : for example, for $p, q \in E(S)$, set $p \leq_{L} q$ if $p=p q$, set $p \leq_{R} q$ if $p=q p$, and set $p \leq q$ if $p=p q=q p$. The orderings $\leq_{L}$ and $\leq_{R}$ are quasi-orders, and $\leq$ is a partial order on $E(S)$. (However $\leq_{L}$ and $\leq_{R}$ need not be anti-symmetric, and so they are not necessarily partial orders.

Further, $(E(S), \leq)$ is not usually an ordered semigroup.) An idempotent is minimal (respectively, maximal) if it is minimal (respectively, maximal) in $(E(S), \leq)$.

By [78, Theorem 1.36], $p \in E(S)$ is minimal if and only if it is minimal for either of the quasi-orderings $\leq_{L}$ or $\leq_{R}$. We say that $p \in$ $E(S)$ is right maximal

if it is maximal in $\left(E(S), \leq_{R}\right)$.

DeFinition 3.4. Let $S$ be a semigroup with a zero o. Then an idempotent $p$ is primitive

if $p \neq o$ and $q=p$ or $q=o$ whenever $q \in E(S)$ with $q \leq p$.

Let $S$ be a semigroup. For subsets $U$ and $V$ of $S$, we set

$$
U V=\{u v: u \in U, v \in V\} .
$$

We write $U x$ for $U\{x\}$, etc. Let $U$ be a subset of a semigroup $S$. Then we define subsets $U_{[n]}$ for $n \in \mathbb{N}$ inductively. Indeed, set $U_{[1]}=U$ and $U_{[n+1]}=U U_{[n]}(n \in \mathbb{N})$. Further, set $U_{[\infty]}=\bigcap\left\{U_{[n]}: n \in \mathbb{N}\right\}$. In the case where $U$ is a subsemigroup of $S$, the family $\left\{U_{[n]}: n \in \mathbb{N}\right\}$ is a decreasing nest of subsemigroups of $S$, and $U_{[\infty]}$ is also a subsemigroup whenever it is non-empty. We note that $E(S) \subset S_{[\infty]}$.

Ideals A left (respectively, right) ideal

in a semigroup $S$ is a non-empty subset $T$ of $S$ such that $S T \subset T$ (respectively, $T S \subset T$ ); a subset which is both a left and right ideal is an ideal. An ideal $I$ in $S$ is prime if $I \neq S$ and $S \backslash I$ is a subsemigroup 
of $S$. Let $T$ be a non-empty subsemigroup of $S$ such that $s, t \in T$ whenever $s, t \in S$ and $s t \in T$. Then $S \backslash T$ is a prime ideal in $S$. A minimal [left] ideal

in $S$ is a [left] ideal which is minimal in the family of [left] ideals of $S$ when this family is ordered by inclusion. Similarly, we define minimum [left] ideals, etc. Let $I$ be an ideal in a semigroup $S$, and let $L$ be a minimal left ideal in $S$. Then $L \subset I$.

Definition 3.5. Let $S$ be a semigroup. Then $F$ is a finite group [left] ideal in $S$ if $F$ is a finite set which is a [left] ideal in $S$ and $F$ is a subgroup of $S$.

The minimum ideal of $S$ (if it exists) is denoted by $K(S)$. The semigroup $S$ is simple

if the only ideal in $S$ is $S$ itself. A principal series of ideals for

$S$ is a chain

$$
S=I_{1} \supsetneq I_{2} \supsetneq \cdots \supsetneq I_{m}=K(S)
$$

where $I_{1}, I_{2}, \ldots, I_{m}$ are ideals in $S$ and there is no ideal of $S$ strictly between $I_{j}$ and $I_{j+1}$ for each $j \in \mathbb{N}_{m-1}$.

The minimum ideal $K(S)$ is called the kernel of $S$ in $[\mathbf{8 1}, \S 3.1]$. A semigroup $S$ is simple if and only if $K(S)=S$; in this case, $S=S_{[2]}$ and $S=S s S(s \in S)$. Let the semigroup $S$ have a principal series as above. Then the factors $I_{j} / I_{j+1}$ are isomorphic in some order to the 'principal factors' of $S$, and so any two principal series have isomorphic sets of factors; see [17] or [81, Exercise 3.8.4].

Let $S$ be a semigroup, and let $I$ be an ideal in $S$. For $s, t \in S$, set $s \sim t$ if $s=t$ or if $s, t \in I$. Clearly $\sim$ is an equivalence relation on $S$; the equivalence class containing $s$ is denoted by $[s]$. We define $[s][t]=[s t] \quad(s, t \in S)$, and obtain a well-defined semigroup operation on the set of equivalence classes. The semigroup $S / I$ so formed is the quotient semigroup;

the equivalence class which is the set $I$ is a zero of $S / I$. Certainly the map $s \mapsto[s], S \rightarrow S / I$, is an epimorphism. See $[\mathbf{8 1}, \S 1.7]$ for further details.

Let $I$ be an ideal in a regular semigroup $S$. Then clearly $I$ and $S / I$ are also regular semigroups.

Proposition 3.6. Let $S$ be a regular semigroup.

(i) Let $p \in S$ be a minimal idempotent. Then $S p$ is a minimal left ideal.

(ii) Suppose that $S$ has just one idempotent. Then $S$ is a group. 
Proof. (i) Let $L$ be a left ideal with $L \subset S p$, and take $s \in L$. Then there exists $t \in S$ with $s t s=s$ and $t s t=t$. Set $q=t s$, so that $q \in E(S) \cap S q$. We have $q \in S s \subset L \subset S p$, so that $q p=q$ and $q \leq_{L} p$. Since $p$ is minimal in $(E(S), \leq), p$ is also minimal in $\left(E(S), \leq_{L}\right)$, and so $p \leq_{L} q$. Hence $S p \subset S q \subset L$, and so $L=S p$. Thus $S p$ is a minimal left ideal.

(ii) $[\mathbf{1 7}$, p. 33, Exercise 4] Let the unique idempotent of $S$ be $e$. For each $s \in S$, there exists $t \in S$ with $s t s=s$, and so $s t=t s=e$. Thus $e$ is the identity of $S$ and $t=s^{-1} \in S$, so that $S$ is a group.

The following is the major structure theorem about semigroups with minimal left ideals which contain an idempotent.

THEOREM 3.7. Let $S$ be a semigroup such that $S$ has a minimal left ideal which contains an idempotent.

(i)

Each left ideal in $S$ contains a minimal left ideal; for each minimal left ideal $L$ in $S$ and each $s \in L, S s=L s=L$; minimal left ideals are pairwise isomorphic.

(ii) Each right ideal in $S$ contains a minimal right ideal; for each minimal right ideal $R$ in $S$ and each $s \in R, s S=s R=R$; minimal right ideals are pairwise isomorphic.

(iii) The minimum ideal $K(S)$ exists; the families of minimal left ideals and of minimal right ideals of $S$ both partition $K(S)$.

(iv) For each minimal right and left ideals $R$ and $L$, there exists $p \in E(S) \cap R \cap L$ such that $R \cap L=R L=p S p$ is a group; these groups are maximal in $K(S)$, are pairwise isomorphic, and the family of these groups partitions $K(S)$; further, $L R=K(S)=S s S(s \in K(S))$.

Proof. This is contained in [78, Corollary 1.47, and Theorems $1.51,1.53,1.54,1.61$, and 1.64]. See also [10].

Proposition 3.8. Let $S$ be a semigroup.

(i) Suppose that $T$ is a subsemigroup of $S$, that both $S$ and $T$ contain a minimal left ideal with an idempotent, and that $K(S) \cap T \neq \emptyset$. Then $K(T)=K(S) \cap T$.

(ii) Suppose that $K(S)$ exists, that $T$ is a semigroup, and that there is an epimorphism $\theta: S \rightarrow T$. Then $K(T)=\theta(K(S))$.

Proof. This is given in [78, Theorem 1.65 and Exercise 1.7.3].

Completely o-simple semigroups We define the special class of completely $o$-simple semigroups, and some related classes of semigroups. 
Definition 3.9. Let $S$ be a semigroup. Suppose that $S$ has a zero o. Then $S$ is o-simple

if $S_{[2]} \neq\{o\}$ and the only ideals in $S$ are $\{o\}$ and $S$, and $S$ is completely o-simple

if it is o-simple and contains a primitive idempotent.

In fact, a completely o-simple semigroup $S$ is regular [81, Lemma 3.2.6]. It follows that, for each $p \in E(S) \backslash\{o\}$, the semigroup $p S p$ is either a group or a group with zero adjoined.

Proposition 3.10. Let $S$ be an o-simple semigroup, and suppose that $p, q \in E(S) \backslash\{o\}$. Then there exists $r \in E(S) \backslash\{o\}$ with $r \leq p$ such that $q S q \cong r S r$.

Proof. [122] Since $S p S=S$, there exist $x_{1}, x_{2} \in S$ such that $x_{1} p x_{2}=q$. Set $y_{1}=q x_{1} p$ and $y_{2}=p x_{2} q$, so that

$$
y_{1} y_{2}=q, \quad q y_{1}=y_{1} p=y_{1}, \quad p y_{2}=y_{2} q=y_{2} .
$$

Set $r=y_{2} y_{1}$. Then $r \in E(S) \backslash\{o\}$ with $r \leq p$. For $z \in S$, set $\theta(z)=y_{1} z y_{2}$. Then $\theta: y_{2} S y_{1} \rightarrow q S q$ is an isomorphism. But we have $y_{2} S y_{1} \supset y_{2} y_{1} S y_{2} y_{1}=r S r$ and $y_{2} S y_{1}=y_{2} q S q y_{1} \subset r S r$, and so $r S r=y_{2} S y_{1}$.

Proposition 3.11. Let $S$ be a completely o-simple semigroup.

(i) Every non-zero idempotent of $S$ is primitive.

(ii) Let $p, q \in E(S)$. Then $p q=q$ if and only if $q p=p$.

(iii) Let $p \in E(S) \backslash\{o\}$. Then $S p$ and $p S$ are minimal non-zero left and right ideals, respectively, and $p S p=G^{o}$ for a group $G$.

(iv) Suppose, further, that $S$ is infinite and that $E(S)$ is finite. Then $S$ contains an infinite group.

Proof. (i) There is a primitive idempotent, say $p$, in $S$.

Take $q \in E(S) \backslash\{o\}$. By Proposition 3.10, $p S p \cong q S q$. Since $p S p$ is either a group or a group with zero adjoined, the same is true of $q S q$, and so $q$ is primitive.

(ii) Suppose that $p q=q$. Then $q p \neq o$ and $q p \leq p$, and so $q p=p$ because $p$ is primitive.

(iii) The first statement has essentially the same proof as that of Proposition 3.6(i), given (i).

Set $G=p S p \backslash\{o\}$. Take $s \in G$, so that $p s p=s$. By (i), $S p$ is a minimal left ideal, and so $S s=S p$; in particular, there exists $u \in S$ with $u s=p$, and we may suppose that $u \in G$. Now take $t \in G$, so that, similarly, there exists $v \in G$ with $t v=p$. We have $(u s)(t v)=p$, 
and so $s t \neq o$, whence $S t \in G$, and so $G$ is a semigroup. The identity of $G$ is $p$. For $s \in G$, there exist $u, v \in G$ with $u s=s v=p$, and then $u=u s v=v$, so that $v=u^{-1} \in G$. Thus $G$ is a group.

(iv) Suppose that $E(S)=\{p, o\}$ for a primitive idempotent $p \in S$. Then $S=p S p=G \cup\{o\}$ for a group $G$, and so the result is immediate.

Now assume inductively that the result holds for each infinite, completely $o$-simple semigroup $T$ with $|E(T)|<|E(S)|$. Consider the completely o-simple semigroup $S p$. If $S p$ is infinite and $S p \not \supset E(S)$, the result follows from the inductive hypothesis. So we may suppose that either $S p$ is finite or $S p \supset E(S)$, and hence that $S p=S$. Similarly, either $p S$ is finite or $p S=S$. Since $S=(S p)(p S)$, either $S p$ or $p S$ is infinite. So we may suppose that $S p=S$. Since $|E(S)|<\infty$, there exist $u, v \in E(S)$ with $u S v=u p S v$ infinite, so that $p S$ is infinite, and hence $p S=S$. But now again $S=p S p=G \cup\{o\}$ for a group $G$, and the result holds for $S$.

The result holds by induction.

We shall also use the following structure theorem in Chapter 10; it is given in [81, Chapter 3].

THEOREM 3.12. Let $S$ be a regular semigroup with finitely many idempotents. Then $K(S)$ exists and $S$ has a principal series

$$
S=I_{1} \supsetneq I_{2} \supsetneq \cdots \supsetneq I_{m-1} \supsetneq I_{m}=K(S)
$$

such that each quotient $I_{j} / I_{j+1}$ is a completely o-simple, regular semigroup with finitely many idempotents. Further, in the case where $S$ is infinite, either $K(S)$ is infinite, or at least one of the quotients $I_{j} / I_{j+1}$ is infinite, and $S$ contains an infinite group.

Proof. The principal series is constructed inductively. Since each quotient $I_{j} / I_{j+1}$ contains a primitive idempotent and $E(S)$ is finite, the construction terminates after finitely many steps.

There exists $j \in \mathbb{N}_{m}$ with $I_{j} / I_{j+1}$ infinite (where $I_{m+1}=\{0\}$ ). By Proposition 3.11(iv), $I_{j} / I_{j+1}$ contains an infinite group $G$. Since $G \cap I_{j+1}=\emptyset$, we may regard $G$ as a subgroup of $I_{j} \subset S$.

Rees semigroups This section discusses 'regular Rees matrix semigroups with a zero over a group $G$ ', which we first define.

These famous semigroups are described in $[\mathbf{1 7}, \S 3.1]$ and $[\mathbf{8 1}, \S 3.2]$; we shall particularly follow the clear account in $[\mathbf{8 1}]$. In fact, we shall define a slight generalization of these semigroups, so that we can introduce notation that will be used in Example 6.18 and Chapter 10 . 
Indeed, let $T$ be a semigroup, and let $G$ be a group that acts bijectively (on the left and right) on $T$ such that $e_{G}$ is the identity on $T$. For $m, n \in \mathbb{N}$, we shall describe a particular semigroup $S$, denoted by

$$
S=\mathcal{M}\left(T, P_{G}, m, n\right) .
$$

Here $P_{G}=\left(a_{i j}\right) \in \mathbb{M}_{n, m}(G)$. For $x \in T, i \in \mathbb{N}_{m}$, and $j \in \mathbb{N}_{n}$, let $(x)_{i j}$ be the element of $\mathbb{M}_{m, n}\left(T^{o}\right)$ with $x$ in the $(i, j)^{\text {th. }}$ place and $o$ elsewhere. As a set, $S$ consists of the collection of all these matrices $(x)_{i j}$. Multiplication in $S$ is given by the formula

$$
(x)_{i j}(y)_{k \ell}=\left(x a_{j k} y\right)_{i \ell} \quad\left(x, y \in T, i, k \in \mathbb{N}_{m}, j, \ell \in \mathbb{N}_{n}\right) ;
$$

associativity is easily verified, as in $[\mathbf{1 7}, \S 3.1]$ and [81, Lemma 3.2.2], and so $S$ is a semigroup.

Similarly, we have the semigroup

$$
\mathcal{M}^{o}\left(T, P_{G}, m, n\right),
$$

where the elements of this semigroup are those of $\mathcal{M}\left(T, P_{G}, m, n\right)$, together with the element $o$, identified with the matrix that has $o$ in each place (so that $o$ is the zero of $\mathcal{M}^{o}\left(T, P_{G}, m, n\right)$ ), and the entries of $P_{G}$ are now allowed to belong to $G^{o}$. The matrix $P_{G}$ is called the sandwich matrix in each case.

The semigroup $\mathcal{M}^{o}\left(T, P_{G}, m, n\right)$ is a Rees matrix semigroup with a zero over $T$ and a subgroup $G$. If $T=G$ is a group, we obtain a Rees matrix semigroup with a zero over $G$, and write

$$
\mathcal{M}^{o}(G, P, m, n)
$$

for our semigroup. (The standard definition in $[\mathbf{1 7}]$ and $[\mathbf{8 1}]$ allows the index sets to be infinite; such semigroups are the generic completely o-simple semigroups [81, Theorem 3.2.3].)

Suppose that $m=n$. Then we write

$$
\mathcal{M}^{o}\left(T, P_{G}, n\right)=\mathcal{M}^{o}\left(T, P_{G}, n, n\right),
$$

etc. Suppose further that $P_{G}=\left(a_{i j}\right)$, where $a_{i i}=e_{G}\left(i \in \mathbb{N}_{n}\right)$ and $a_{i j}=o(i \neq j)$, so that $P=I_{G}(n)$ is the $n \times n$ identity matrix.

Then we set

$$
\mathcal{M}^{o}(T, n)=\mathcal{M}^{o}\left(T, P_{G}, n\right) .
$$

Suppose that $G=\{e\}$. Then we write $\mathcal{M}^{o}(P, m, n)$ for the semigroup $\mathcal{M}^{o}(G, P, m, n)$, etc.

The above sandwich matrix $P_{G}$ is regular

if every row and column contains at least one entry in $G$. The semigroup $\mathcal{M}^{o}(G, P, m, n)$ is regular as a semigroup if and only if the sandwich matrix is regular [17, Lemma 3.1]. 
Two semigroups $\mathcal{M}^{o}\left(G,\left(a_{i j}\right), m, n\right)$ and $\mathcal{M}^{o}\left(G,\left(b_{i j}\right), m, n\right)$ are isomorphic if there exist $s_{1}, \ldots, s_{n}$ and $t_{1}, \ldots, t_{m}$ in $G$ with

$$
b_{i j}=s_{i} a_{i j} t_{j} \quad\left(i \in \mathbb{N}_{n}, j \in \mathbb{N}_{m}\right) .
$$

In particular, we are allowed to exchange any two distinct columns and any two distinct rows of $P$ at will [17, Corollary 3.12]. For example, up to isomorphism, the $2 \times 2$ regular sandwich matrices over a group $G$ have one of the three forms

$$
\left(\begin{array}{ll}
e & o \\
o & e
\end{array}\right), \quad\left(\begin{array}{ll}
e & o \\
e & e
\end{array}\right), \quad\left(\begin{array}{ll}
e & s \\
e & e
\end{array}\right),
$$

where $e=e_{G}$ and $s \in G$.

Let $G$ be a group, let $\{e\}$ be the group with just one element, and let $\theta: G \rightarrow\{e\}$ be the epimorphism. Then there is an obvious epimorphism

$$
\theta: \mathcal{M}^{o}(G, P, m, n) \rightarrow \mathcal{M}^{o}(\theta(P), m, n) .
$$

Let $S=\mathcal{M}^{o}\left(T, P_{G}, m, n\right)$, as above, with $P_{G}$ regular. We set

$$
N\left(P_{G}\right)=\left\{(j, k) \in \mathbb{N}_{n} \times \mathbb{N}_{m}: a_{j k} \neq o\right\}
$$

and

$$
Z\left(P_{G}\right)=\left\{(j, k) \in \mathbb{N}_{n} \times \mathbb{N}_{m}: a_{j k}=o\right\} .
$$

For $i \in \mathbb{N}_{m}$ and $j \in \mathbb{N}_{n}$, let $X_{i j}=\left\{(x)_{i j}: x \in G\right\}$ and $e_{i j}=\left(e_{G}\right)_{i j}$. The elements $e_{i j}$ are the matrix units

of $S$. Then the sets $X_{i j}$ are pairwise disjoint, and $S$ is the union of these sets, together with $\{o\}$. If $a_{j i}=o$, the product of any two elements in $X_{i j}$ is $o$. If $a_{j i} \in G, X_{i j}$ is (isomorphic to) the semigroup $T$ by the map $(x)_{i j} \mapsto x a_{j i}$, and $T$ is infinite if $S$ is infinite.

An idempotent other than $o$ of $\mathcal{M}^{o}(G, P, m, n)$ has the form $\left(a_{j k}^{-1}\right)_{k j}$, where $(j, k) \in N(P)$, and so

$$
|E(S)|=|N(P)|+1 .
$$

In particular, in the case where $m=n$, set $P=I_{G}(n)=\sum_{i=1}^{n} e_{i i}$ and $S=\mathcal{M}^{o}(G, n)$. Then $|E(S)|=n+1$.

The result that we shall require is essentially the famous classification theorem of Rees [121], which is contained in [17, Lemma 2.46 and Theorem 3.5] and [81, Theorem 3.2.3]; see Chapter 4 for the construction of the semigroup algebras of these semigroups and Chapter 10 for our application of this theorem.

Theorem 3.13. Let $G$ be a group, let $m, n \in \mathbb{N}$, and let $P$ be a regular sandwich matrix. Then $\mathcal{M}^{\circ}(G, P, m, n)$ is a completely o-simple semigroup with finitely many idempotents. 
Let $S$ be an infinite, completely o-simple semigroup with finitely many idempotents. Then there is an infinite group $G$ which is a maximal subgroup of $S$ such that $G^{o}=p S p$ for each $p \in E(S) \backslash\{o\}$, and $S$ is isomorphic as a semigroup to a regular Rees matrix semigroup with a zero $\mathcal{M}^{o}(G, P, m, n)$.

Cancellative semigroups Let $S$ be a semigroup. For $s \in S$, define

$$
L_{s}(t)=s t, \quad R_{s}(t)=t s \quad(t \in S) .
$$

Let $U$ be a non-empty subset of $S$. Then

$$
\left\{\begin{array}{l}
s^{-1} U=L_{s}^{-1}(U)=\{t \in S: s t \in U\}, \\
U s^{-1}=R_{s}^{-1}(U)=\{t \in S: t s \in U\} .
\end{array}\right.
$$

We write $s^{-1} x$ for the set $s^{-1}\{x\}$, etc. Further, let $V$ be another nonempty subset of $S$. Then

$$
\left\{\begin{array}{l}
V^{-1} U=\bigcup\left\{s^{-1} U: s \in V\right\}, \\
U V^{-1}=\bigcup\left\{U s^{-1}: s \in V\right\} .
\end{array}\right.
$$

Suppose that $G$ is a group and that $U \subset G$. Then

$$
U^{-1}=\left\{u^{-1}: u \in U\right\} .
$$

Definition 3.14. Let $S$ be a semigroup. An element $s \in S$ is left (respectively, right) cancellable if $L_{s}$ (respectively, $R_{s}$ ) is injective on $S$, and $s$ is cancellable if it is both left cancellable and right cancellable.

The semigroup $S$ is left (respectively, right) cancellative if each element is left (respectively, right) cancellable, and cancellative

if each element is cancellable.

The semigroup $S$ is weakly left (respectively, right) cancellative if $s^{-1} F$ (respectively, $F s^{-1}$ ) is finite

for each $s \in S$ and each finite subset $F$ of $S$, and $S$ is weakly cancellative if it is both

weakly left cancellative and weakly right cancellative.

Certainly, each subsemigroup of a group is cancellative. Let $S$ be a cancellative semigroup. In the case where $S$ is abelian, $S$ is a subsemigroup of a group $G$ with $|G|=|S|$ [19, Proposition 1.2.10]. However, in general, $S$ is not necessarily a subsemigroup of any group: rather complicated necessary and sufficient conditions for this, and examples for which the conditions fail, are given in $[\mathbf{1 7}$, Chapter 10] and $[\mathbf{1 0 2}$, Chapter IX]. 
Proposition 3.15. Let $S$ be an infinite, cancellative semigroup. Then $|E(S)| \leq 1$ and either $S$ contains a countable, infinite subgroup or $S$ contains a subsemigroup isomorphic to $\mathbb{N}$.

Proof. First suppose that $p \in E(S)$. For each $s \in S$, we have $p^{2} s=p s$ and $s p^{2}=s p$, and so $p s=s p=s$ because $S$ is cancellative. Thus $p$ is the identity of $S$.

In the case where $\langle s\rangle$ is finite for each $s \in S$, necessarily each $\langle s\rangle$ is a finite subgroup of $S$; the identity of each group $\langle s\rangle$ is an idempotent, and so is equal to the identity $e_{S}$ of $S$. Thus each $s \in S$ has an inverse in $S$, and so $S$ is a group. Certainly $S$ contains a countable, infinite subgroup

In the case where $\left\langle s_{0}\right\rangle$ is infinite for some $s_{0} \in S$, take $T=\left\langle s_{0}\right\rangle$. Then $T$ is a subsemigroup isomorphic to $\mathbb{N}$.

Let $S$ be a regular semigroup. Then the following are equivalent [81, Exercise 2.6.11]: (a) $|E(S)|=1$; (b) $S$ is cancellative; (c) $S$ is a group.

Let $S$ be a semigroup, and let $p \in E(S)$. Then $p$ is left (respectively, right) cancellable if and only if $p$ is a left (respectively, right) identity for $S$.

Proposition 3.16. Let $S$ be an infinite, cancellative semigroup. Then there is a sequence in $S$ which has distinct finite products.

Proof. This is [78, Lemma 6.31].

Proposition 3.17. Let $S$ be a weakly left cancellative semigroup, and let $T$ be an infinite subset of $S$. Then there exists a subsemigroup $U$ of $S$ with $U \supset T$, with $|U|=|T|$, and such that $U s \cap U=\emptyset(s \in S \backslash U)$.

Proof. For an infinite subset $W$ of $S$, define

$$
i(W)=\{s \in S: W s \cap W \neq \emptyset\} .
$$

Since $S$ is weakly left cancellative, we have $|i(W)| \leq|W|$, and so we have $|W \cup i(W)|=|\langle W \cup i(W)\rangle|=|W|$. We set $U_{0}=T$ and also $U_{n+1}=\left\langle U_{n} \cup i\left(U_{n}\right)\right\rangle$ for $n \in \mathbb{N}$. Then $U:=\bigcup\left\{U_{n}: n \in \mathbb{N}\right\}$ has the required properties.

Let $\left\{S_{i}: i \in I\right\}$ be a family of semigroups. Then the semigroup

$$
\prod S_{i}=\prod\left\{S_{i}: i \in I\right\}
$$

where the semigroup operation is specified by setting $\left(s_{i}\right)\left(t_{i}\right)=\left(s_{i} t_{i}\right)$, is the direct product of the family. In the case where each $S_{i}$ is unital,

$$
\bigoplus S_{i}=\bigoplus\left\{S_{i}: i \in I\right\}
$$


is the direct sum

of the family: here the elements of $\bigoplus S_{i}$ are sequences $\left(s_{i}: i \in I\right)$ in $\prod S_{i}$ such that $s_{i}$ is the identity of $S_{i}$ for all save finitely many values of $i \in I$.

Let $\left\{S_{i}: i \in I\right\}$ be a family of weakly cancellative, unital semigroups. Then $\bigoplus\left\{S_{i}: i \in I\right\}$ is also weakly cancellative.

We now introduce an apparently new condition on semigroups.

Definition 3.18. A semigroup $S$ is nearly right cancellative if there is a subset $X$ of $S$ such that $|X|=|S|$ and also, for each $s, t \in S$ with $s \neq t$, the set $\{x \in X: s x=t x\}$ is finite; such a set $X$ is a witness in $S$. The semigroup $S$ is nearly left cancellative if $S^{\text {op }}$ is nearly right cancellative, and nearly cancellative if it is both nearly right cancellative and nearly left cancellative.

Clearly a [right] cancellative semigroup is nearly [right] cancellative. We shall later give examples of semigroups that are weakly cancellative and nearly cancellative, but not right cancellative; some later results apply to exactly the class of weakly cancellative and nearly right cancellative semigroups. Note that a subsemigroup of a nearly right cancellative semigroup is not necessarily nearly right cancellative, but, for each subset $T$ of a nearly right cancellative semigroup $S$, there is a nearly right cancellative subsemigroup $U$ of $S$ with $|U|=|T|$.

The following result shows that there are many nearly right cancellative semigroups.

Proposition 3.19. (i) Let $S$ be an infinite semigroup. Then there is a nearly cancellative semigroup $T$ containing $S$ as a subsemigroup and with $|T|=|S|$.

(ii) Let $\kappa$ be an infinite cardinal, and let $\left\{S_{\sigma}: \sigma<\kappa\right\}$ be a family of unital semigroups with $2 \leq\left|S_{\sigma}\right| \leq \kappa(\sigma<\kappa)$. Then $\bigoplus\left\{S_{\sigma}: \sigma<\kappa\right\}$ is nearly cancellative.

Proof. (i) Let $G$ be any group with $|G|=|S|$, and set $T=S^{\#} \oplus G$. Take $X=\left\{\left(e_{S}, x\right): x \in G\right\}$. Then the elements of $X$ are cancellable and $|X|=|T|$, and so $X$ is a witness to the fact that $T$ is nearly cancellative.

(ii) Set $S=\bigoplus\left\{S_{\sigma}: \sigma<\kappa\right\}$. For each $\sigma<\kappa$, choose $s_{\sigma} \in S_{\sigma}$ with $s_{\sigma} \neq e_{S_{\sigma}}$, and define $x_{\sigma} \in S$ for $\sigma<\kappa$ to be the sequence with $s_{\sigma}$ in the $\sigma^{\text {th. }}$ coordinate, and with $e_{S_{\tau}}$ in the $\tau^{\text {th. }}$ coordinate for each $\tau<\kappa$ with $\tau \neq \sigma$. Set $X=\left\{x_{\sigma}: \sigma<\kappa\right\}$, so that $|X|=\kappa=|S|$. The set $X$ is a witness to the fact that $S$ is nearly cancellative. 
Almost left disjoint subsets We first pause to explain some notation that will be used at several future points; it involves the enumeration of a semigroup.

Let $S$ be an infinite semigroup with $|S|=\kappa$; we may suppose for convenience that $S$ has an identity $e_{S}$. We enumerate $S$ as a sequence $\left(s_{\alpha}: \alpha<\kappa\right)$, where $s_{0}=e_{S}$. For $s=s_{\alpha}$ and $t=s_{\beta}$ in $S$, set $s \preccurlyeq t$ if $\alpha \leq \beta$ in $\kappa$ and set $s \prec t$ if $\alpha<\beta$ in $\kappa$. Thus $(S, \preccurlyeq)$ is a totally ordered set, but in general it is not the case that $(S, \preccurlyeq)$ is an ordered semigroup.

For $t \in S$ and a subset $F$ of $S$, set

$$
[t]=\{s \in S: s \preccurlyeq t\}, \quad[F]=\bigcup\{[t]: t \in F\} .
$$

Further, for $\alpha \leq \beta<\kappa$, set $\left[s_{\alpha}, s_{\beta}\right]=\left\{s_{\gamma} \in S: \alpha \leq \gamma \leq \beta\right\}$, with similar notation for other 'intervals' in $S$. For a subset $F$ of $S$, the maximum and supremum of $F$ are defined with respect to $\preccurlyeq$.

TheOREM 3.20. Let $S$ be an infinite semigroup of cardinality $\kappa$ such that $S$ is weakly cancellative and right cancellative. Then each subset $X$ of $S$ such that $|X|=\kappa$ contains a subset $F$ such that $|F|=\kappa$ and

$$
\left|s^{-1} F \cap t^{-1} F\right|<\kappa, \quad|s F \cap t F|<\kappa \quad(s, t \in S, s \neq t) .
$$

Proof. We may suppose that $S$ is unital, with identity $e_{S}$, and that $e_{S} \in X$. Set $\kappa=|S|$, and enumerate $S$ as $\left\{s_{\alpha}: \alpha<\kappa\right\}$, with $s_{0}=e_{S}$, and set $S_{\alpha}=\left\{s_{0}, s_{1}, \ldots, s_{\alpha}\right\}$ for each $\alpha<\kappa$.

We shall define subsets $F_{\alpha}$ of $X$ inductively for each $\alpha<\kappa$ in such a way that $F_{n}$ is finite for $n<\omega$ and $\left|F_{\alpha}\right|=|\alpha|$ for each $\alpha \in[\omega, \kappa)$.

Set $x_{0}=s_{0}$ and $F_{0}=\left\{x_{0}\right\}$.

Now assume that $F_{\alpha}$ has been specified, where $\alpha<\kappa$. Set

$$
U_{\alpha}=\left(S_{\alpha}^{-1} S_{\alpha} F_{\alpha}\right) \cup\left(S_{\alpha} S_{\alpha}^{-1} F_{\alpha}\right) \subset S .
$$

Then $U_{\alpha}$ is finite for $\alpha<\omega$ and $\left|U_{\alpha}\right|=\left|F_{\alpha}\right|=|\alpha|$ for $\omega \leq \alpha<\kappa$ because $S$ is weakly cancellative, and also $U_{\alpha} \supset\left(S_{\alpha} \cup F_{\alpha}\right)$. Choose $x_{\alpha+1} \in X \backslash U_{\alpha}$, and set $F_{\alpha+1}=F_{\alpha} \cup\left\{x_{\alpha+1}\right\}$, so that $\left|F_{\alpha+1}\right|$ has the correct value in each case. In the case where $\alpha<\kappa$ and $\alpha$ is a non-zero limit ordinal, define $F_{\alpha}=\bigcup\left\{F_{\beta}: \beta<\alpha\right\}$. Again $\left|F_{\alpha}\right|=|\alpha|$.

This completes the inductive construction of the sets $F_{\alpha}$ for $\alpha<\kappa$.

We define $F=\bigcup\left\{F_{\beta}: \beta<\kappa\right\}$. Certainly $F \subset X$ and $|F|=\kappa$. Fix distinct elements $s, t \in S$, and choose $\alpha<\kappa$ with $s, t \in S_{\alpha}$.

First take $u \in s^{-1} F \cap t^{-1} F$, say $s u=x_{\beta}, t u=x_{\gamma}$, where $\beta \leq \gamma<\kappa$. Then in fact $\beta<\gamma$ because $u$ is right cancellable in $S$. Assume towards a contradiction that $\beta \geq \alpha$. Then

$$
x_{\gamma}=t u \in t s^{-1} F_{\beta} \subset S_{\beta} S_{\beta}^{-1} F_{\beta} \subset U_{\beta},
$$


a contradiction. Thus $\beta<\alpha$, and so $u \in S_{\alpha}^{-1} S_{\alpha} F_{\alpha} \subset U_{\alpha}$. We have shown that $s^{-1} F \cap t^{-1} F \subset U_{\alpha}$, and so $\left|s^{-1} F \cap t^{-1} F\right| \leq \alpha<\kappa$.

Second take $u \in s F \cap t F$, say $u=s x_{\beta}=t x_{\gamma}$, with $\beta \leq \gamma$. Then in fact $\beta<\gamma$ because $x_{\beta}$ is right cancellable. Assume that $\beta \geq \alpha$. Then $x_{\gamma} \in t^{-1} s F_{\beta} \subset U_{\beta}$, a contradiction. Thus $\beta<\alpha$, and so $u \in S_{\alpha}^{2} F_{\alpha}$. We have shown that $s F \cap t F \subset S_{\alpha}^{2} F_{\alpha}$, and so $|s F \cap t F| \leq \alpha<\kappa$.

The theorem is proved.

We introduce a variation of a well-known concept (see [78, p. 237]).

Definition 3.21. Let $S$ be an infinite semigroup. A subset $F$ of $S$ is almost left disjoint if $F$ is countable and infinite and if

$s^{-1} F \cap t^{-1} F$ is finite whenever $s, t \in S$ with $s \neq t$.

For example, let $\left(n_{k}\right)$ be a sequence in $\mathbb{N}$ such that $n_{k+1}-n_{k} \rightarrow \infty$ as $k \rightarrow \infty$. Then the set $\left\{n_{k}: k \in \mathbb{N}\right\}$ is an almost left disjoint subset of $(\mathbb{N},+)$. On the other hand, $\mathbb{N}_{\vee}$ contains no almost left disjoint subsets; see Example 3.36.

TheOREM 3.22. Let $G$ be an infinite group. Then each infinite subset of $G$ contains an almost left disjoint subset.

Proof. Let $X$ be an infinite, countable subset of $G$, and take $H$ to be the subgroup of $G$ generated by $X$, so that $H$ is also countable. By Theorem 3.20, there is a countable subset $F$ of $X$ such that $F$ is almost left disjoint in $H$. Take $s, t \in G$ with $s \neq t$, and suppose that $s F \cap t F \neq \emptyset$. Then $s H \cap t H \neq \emptyset$, and so the left cosets $s H$ and $t H$ are equal. Hence $s=t h$ for some $h \in H$. Suppose that $u \in s F \cap t F$. Then $h^{-1} t^{-1} u \in F$ and $t^{-1} u \in F$, and so $t^{-1} u \in h F \cap F$, a finite set. Thus $s F \cap t F \subset t(h F \cap F)$, a finite set. Hence $F$ is almost left disjoint in $G$.

THEOREM 3.23. Let $S$ be an infinite, cancellative semigroup. Then $S$ contains an almost left disjoint subset.

Proof. By Proposition 3.15, $S$ contains a countable, infinite subsemigroup $T$ such that $T$ is either a group or isomorphic to $\mathbb{N}$. The set $T$ has the property that, for each $r, s \in T$ with $r \neq s$, there exists $t \in T$ such that either $t r=s$ or $t s=r$. Clearly $T$ contains an almost left disjoint subset, say $F$, for $T$ such that $y F \cap F$ is finite for each $y \in T \backslash\left\{e_{T}\right\}$.

Assume towards a contradiction that there exist $s, t \in S$ with $s \neq t$ such that $s^{-1} F \cap t^{-1} F$ is infinite. Choose $x \in s^{-1} F \cap t^{-1} F$. Then $s x, t x \in F$, and $s x \neq t x$. Thus there exists $y \in T \backslash\left\{e_{T}\right\}$ with $y s x=t x$, 
say. But now $y s=t$ and $y s z=t z \in y F \cap F$ for every $z \in s^{-1} F \cap t^{-1} F$, and so $y F \cap F$ is infinite, a contradiction. Thus $F$ is an almost left disjoint set for $S$.

The above result is trivially false if $S$ is just abelian and weakly cancellative. Indeed, set $S=\left\{e_{1}, e_{2}\right\} \cup \mathbb{N}$ as a set, and define

$$
\left\{\begin{aligned}
e_{i} \cdot e_{j} & =e_{i \vee j} \quad(i, j=1,2), \\
e_{i} \cdot m & =m \cdot e_{i}=m \quad(m \in \mathbb{N}, i=1,2), \\
m \cdot n & =m+n \quad(m, n \in \mathbb{N}) .
\end{aligned}\right.
$$

Then $(S, \cdot)$ is a countable, abelian, weakly cancellative semigroup, but $e_{1}^{-1} \cdot F \cap e_{2}^{-1} \cdot F \supset F$ for each subset $F$ of $\mathbb{N}$, and so $S$ contains no almost left disjoint subset. The above result is also trivially false if $S$ is either just left or right cancellative or if $S$ is just abelian, weakly cancellative, and nearly cancellative; see Examples 3.30 and 3.40, respectively.

Topological semigroups The following is a standard definition.

DeFinition 3.24. A semigroup $S$ which is also a topological space is a left (respectively, right) topological semigroup if $L_{t}$ (respectively, $R_{t}$ ) is continuous for each $t \in S$, a semi-topological semigroup if the product map $m_{S}$ is separately continuous, and a topological semigroup if $m_{S}$ is continuous.

A group $G$ is a topological group if it is a topological semigroup and, further, the map $s \mapsto s^{-1}, G \rightarrow G$, is also continuous.

For the theory of topological semigroups, see [10] and, especially, [78, Chapter 2]; we shall seek to follow the notation of [78] throughout.

We shall see later that the following two theorems apply in particular in the case where $V=(\beta S, \square)$ for a semigroup $S$; see Theorem 6.1(i). Clause (i) shows that the structure theorem 3.7 applies to compact, right topological semigroups.

TheOREM 3.25. Let $V$ be a compact, right topological semigroup.

Then $V$ has a minimal left ideal which contains an idempotent, and so the structure theorem 3.7 applies.

In particular, the minimum ideal

$K(V)$ exists and $E(V) \neq \emptyset$. Further:

(i) minimal left ideals of $V$ are closed in $V$;

(ii) the closure of each right ideal in $V$ is a right ideal;

(iii) $V$ contains right maximal idempotents. 
Proof. The main claim that $V$ has a minimal left ideal which contains an idempotent is [78, Corollary 2.6]; (ii) and (iii) are [78, Theorems 2.15 and 2.12], respectively.

By [78, Example 2.16], the closure of a left ideal in $V$ is not necessarily a left ideal.

THEOREM 3.26. Let $V$ be a compact, right topological semigroup, and let $p \in E(V)$. Then the following conditions on $p$ are equivalent: (a) $V p$ is a minimal left ideal; (b) $p V$ is a minimal right ideal; (c) $p V p$ is a subgroup of $V$; (d) $p V p$ is the maximal subgroup of $V$ with $p$ as an identity; (e)

$p$ is a minimal idempotent; (f) $p \in K(V)$; (g) $K(V)=V p V$.

Proof. These (and other equivalences) are contained in $[\mathbf{7 8}$, Theorem 2.9] and [10].

We conclude this subsection by introducing a certain class of semigroups.

Definition 3.27. Let $S$ be a semigroup. Then $S$ is maximally almost periodic if there is a compact topological group $G$ and a monomorphism from $S$ into $G$.

A group $H$ is maximally almost periodic if and only if the finitedimensional, irreducible, unitary representations of $H$ on a Hilbert space separate the points of $H$; this is the standard definition of such a group [114, Theorem 12.4.15]. Each cancellative, abelian semigroup and each subsemigroup of a free group is maximally almost periodic.

\section{Characters and semi-characters}

Definition 3.28. Let $S$ be a semigroup.

$A$ semi-character (respectively, character) on $S$ is a map $\varphi: S \rightarrow \overline{\mathbb{D}}$ (respectively, $\varphi: S \rightarrow \mathbb{T}$ ) such that

$$
\varphi(s t)=\varphi(s) \varphi(t) \quad(s, t \in S) \quad \text { and } \quad \varphi \neq 0 .
$$

The spaces of semi-characters and characters on $S$ are denoted by $\Phi_{S}$ and $\Psi_{S}$, respectively.

See [10, Definition 1.18], [35, Definition 1.1.9], and [72, Definition $(22.15)]$, but note that our definition is different from that given in [9, page 92], which requires a further condition. Often, the space of semi-characters on a semigroup $S$ is denoted by $H(S)$. 
There is always one character on $S$, namely the map

$$
1: s \mapsto 1, \quad S \rightarrow \mathbb{T} ;
$$

this is the augmentation character.

In general, the augmentation characters may be the only semicharacter on $S$.

Let $\varphi, \psi \in \Phi_{S} \cup\{0\}$. Then we define $\varphi \cdot \psi \in \Phi_{S} \cup\{0\}$ by

$$
(\varphi \cdot \psi)(s)=\varphi(s) \psi(s) \quad(s \in S) .
$$

Clearly $\Phi_{S} \cup\{0\}$ is an abelian semigroup with identity 1 with respect to this product $\cdot$. Let $\varphi \in \Phi_{S}$, and define $\bar{\varphi}: s \mapsto \overline{\varphi(s)}, S \rightarrow \mathbb{C}$. Then $\bar{\varphi} \in \Phi_{S}$.

Let $p$ be an idempotent in a semigroup $S$. Then $\varphi(p)=0$ or $\varphi(p)=1$ for each $\varphi \in \Phi_{S}$. Set

Let $\varphi$ be a semi-character on $S$, and let $I$ be a prime ideal in $S$.

$$
\psi(s)=\varphi(s) \quad(s \in S \backslash I) \quad \text { and } \quad \psi(s)=0 \quad(s \in I),
$$

so that $\psi=\varphi \cdot \chi_{S \backslash I}$. Then $\psi \in \Phi_{S}$. Conversely, for each $\varphi \in \Phi_{S}$, the set $\{s \in S: \varphi(s)=0\}$ is a prime ideal in $S$.

Let $S$ be a semigroup. The space $\Phi_{S} \cup\{0\}$ is compact and $\Phi_{S}$ is locally compact with respect to the topology of pointwise convergence on $S$. Further, $\left(\Phi_{S} \cup\{0\}, \cdot\right)$ is a compact topological semigroup, called the dual semigroup to $S$;

see [8] and [35]. For example, $\Phi_{S}=\overline{\mathbb{D}}$ when $S=\mathbb{Z}^{+}$. The space $\Psi_{S}$ is a compact subspace and a subgroup of the compact semigroup $\Phi_{S} \cup\{0\}$ (and $\left.\Psi_{S} \subset \Phi_{S}\right)$. Indeed, $\left(\Psi_{S}, \cdot\right)$ is a compact topological group.

Let $G$ be a group. Then every semi-character on $G$ is a character, and so $\Psi_{G}=\Phi_{G}$. Let $N$ be the commutator subgroup

of $G$ (so that $N$ is generated by elements of the form $s t s^{-1} t^{-1}$ for $s, t \in G)$, and let $H$ be the quotient group $G / N$, so that $H$ is an abelian group. Then $\Phi_{G}$ is just the dual group $\widehat{H}$ of $H$. Let $(S,+)$ be a cancellative, abelian semigroup. Then $S$ is a subsemigroup of an abelian group $(G,+)$ such that each element of $G$ has the form $s-t$ for some $s, t \in S$. Let $\varphi \in \Psi_{S}$, and define $\widetilde{\varphi}$ on $G$ by setting $\widetilde{\varphi}(s-t)=\varphi(s) \varphi(t)^{-1}$. Then $\widetilde{\varphi}$ is a well-defined character on $G$ and $\widetilde{\varphi} \mid S=\varphi$. In this way we can identify $\Psi_{S}$ with $\Psi_{G}=\widehat{G}$.

Examples of semigroups We conclude this section with a collection of examples of semigroups. 
ExAmple 3.29. The standard example of semigroup that we have in mind is $(\mathbb{N},+)$, just denoted by $\mathbb{N}$; similarly we shall consider the group $\mathbb{Z}=(\mathbb{Z},+)$. A further semigroup is $(\mathbb{N}, \cdot)$; this latter semigroup is not finitely generated. All these semigroups are cancellative.

EXAMPLE 3.30. Let $S$ be an infinite set with the product given by

$$
s t=t \quad(s, t \in S)
$$

so that $S$ is a right zero semigroup.

Then $S$ is a semigroup which is right cancellative, but not weakly left cancellative. Each element of $S$ is a left identity, but there are no right identities. Let $F \in \mathcal{P}(S)$. Then $s^{-1} F=F(s \in S)$, and so $S$ contains no almost left disjoint subset.

Similarly, a left zero semigroup

has the product specified by setting

$$
s t=s \quad(s, t \in S)
$$

now $S$ is left cancellative. Let $F \in \mathcal{P}(S)$. Then $s^{-1} F=S(s \in F)$, and so $S$ contains no almost left disjoint subset.

EXAmPle 3.31. Let $S$ be an infinite set, and let $p \in S$. Set

$$
s t=p \quad(s, t \in S) .
$$

Then $S$ is a semigroup; it is a trivial semigroup at

$p$. The element $p$ is a zero of this semigroup.

EXAMPLE 3.32. Let $T=\mathbb{Z}^{2}$, and define

$$
\left(m_{1}, n_{1}\right) \cdot\left(m_{2}, n_{2}\right)=\left(m_{1}+m_{2}, n_{2}\right) \quad\left(m_{1}, n_{1}, m_{2}, n_{2} \in \mathbb{Z}\right) .
$$

Then $(T, \cdot)$ is a non-abelian semigroup which is left cancellative, but not weakly right cancellative. Each infinite subset of $T$ contains an almost left disjoint subset;

the elements of the form $(0, n)$, for $n \in \mathbb{Z}$, are idempotent of $T$. In fact, $T=(\mathbb{Z},+) \times S$, where $S$ is a right zero semigroup on $\mathbb{N}$. For more $(T, \cdot)$, see Examples 7.31 and 10.9.

ExAmple 3.33. Let $S=\mathbb{N} \times\{0,1\}$, with the operation

$$
(m, i) \cdot(n, j)=(m+n, 0) \quad(m, n \in \mathbb{N}, i, j \in\{0,1\}) .
$$

Then $S$ is an abelian, countable, weakly cancellative semigroup. However $S$ is not nearly cancellative because we have $(1,0) \neq(1,1)$, but $(1,0) \cdot x=(1,1) \cdot x$ for each $x \in S$. For more on this example, see Example 7.34. 
EXAmple 3.34. Let $S=(\mathbb{N} \times \mathbb{N}) \cup\{o\}$, with the product such that, for $(m, i),(n, j) \in \mathbb{N} \times \mathbb{N}$, we have $(m, i) \cdot(n, j)=(m+n, i)$ when $j=i$ and $(m, i) \cdot(n, j)=o$ when $i \neq j$; also set $s \cdot o=o \cdot s=o(s \in S)$. Then $(S, \cdot)$ is an abelian semigroup with a zero, $o$. For $j \in \mathbb{N}$, the set $S_{j}=\mathbb{N} \times\{j\}$ is a subsemigroup of $S$, and so $S=\left(\bigcup_{j \in \mathbb{N}} S_{j}\right) \cup\{o\}$, where $\left\{S_{j}: j \in \mathbb{N}\right\}$ is a countable family of pairwise disjoint infinite subgroups, and $S_{i} S_{j}=\{o\}$ when $i \neq j$. For more on this example, see Example 12.19.

ExAmPle 3.35. Let $S$ be an infinite semigroup, and set $T=S \times S$ as a set. Define

$$
(a, x) \cdot(b, y)=(a b, a y) \quad(a, b, x, y \in S) .
$$

Then $(T, \cdot)$ is a semigroup which is not weakly right cancellative; it is left cancellative whenever $S$ is left cancellative.

EXAMPLE 3.36. Let $S$ be the set $\mathbb{N}$, with the product

$$
(m, n) \mapsto m \vee n=\max \{m, n\}, \quad \mathbb{N} \times \mathbb{N} \rightarrow \mathbb{N}
$$

Then $S$ is a semigroup with the identity 1, and $S$ is countable, abelian, and weakly cancellative. However $S$ is not nearly cancellative, and $S$ does not contain any almost disjoint subset. Clearly $E(S)=S$. This semigroup will be denoted by $\mathbb{N}_{\vee}$.

EXAmple 3.37. Let $S$ be the set $\mathbb{N}$, with the product

$$
(m, n) \mapsto m \wedge n=\min \{m, n\}, \quad \mathbb{N} \times \mathbb{N} \rightarrow \mathbb{N} .
$$

Then $S$ is a countable, abelian semigroup, and 1 acts as a zero; $S$ is nearly cancellative, but $S$ is not weakly cancellative. Clearly $E(S)=S$, but $S$ does not have an identity. This semigroup will be denoted by $\mathbb{N}_{\wedge}$.

ExAmple 3.38. Let $S_{n}=\mathbb{N}_{\vee}$ for $n \in \mathbb{N}$, and set

$$
S=\bigoplus\left\{S_{n}: n \in \mathbb{N}\right\} .
$$

Then $S$ is weakly cancellative by Proposition 3.19(ii), but $S$ is not cancellative; $S$ is nearly cancellative. The semigroup $S$ is isomorphic to the set $\mathbb{N}$, with the semigroup operation specifed by taking $m \cdot n$ to be the lowest common multiple of $m$ and $n$ for $m, n \in \mathbb{N}$. 
EXAmple 3.39. Let $S=\mathbb{M}_{n}(\mathbb{N})$, the set of $n \times n$-matrices with coefficients in $\mathbb{N}$. Then $S$ is a semigroup with respect to the product of matrices. This semigroup is weakly cancellative and nearly cancellative (taking the witness $X$ to be the set of matrices in $S$ with non-zero determinant), but the semigroup $S$ is neither left nor right cancellative whenever $n \geq 2$.

ExAmple 3.40. Let $S$ be a non-empty set, and let $V=\mathcal{P}_{f}(S)$, with the semigroup operation

$$
(Y, Z) \mapsto Y \cup Z, \quad V \times V \rightarrow V .
$$

Then $V$ is is abelian, weakly cancellative and nearly cancellative (taking the witness $X$ to be the set of singletons in $V$ ), but $V$ is not cancellative. Each element is an idempotent, and so $S$ is a semilattice. This semigroup does not have any almost left disjoint subset.

ExAmple 3.41. Let $S$ be an infinite set, and set $T=S^{S}$, with composition $\circ$ of functions as the semigroup operation; let $T$ have the product topology. Then $T$ is a topological semigroup [78, Corollary $2.3]$.

Let $X$ be the subset of $T$ consisting of the surjective functions. For each $f, g \in T$ with $f \neq g$, the set

$$
\{h \in X: f \circ h=g \circ h\}
$$

is empty; further, $|X|=2^{\kappa}=|T|$, where $\kappa=|S|$. This shows that $T$ is nearly right cancellative; clearly the semigroup $T$ is not weakly cancellative.

EXAMPLE 3.42. The free semigroup on 2 symbols is $\mathbb{S}_{2}$;

$\mathbb{S}_{2}$ is cancellative and a subsemigroup of $\mathbb{F}_{2}$, the free group on 2 symbols.

Each element $w$ in $\mathbb{F}_{2}$ has the reduced form

$$
w=a^{m_{1}} b^{n_{1}} \cdots a^{m_{k}} b^{n_{k}},
$$

where $k \in \mathbb{N}, m_{1}, n_{1}, \ldots, m_{k}, n_{k} \in \mathbb{Z}$, and $n_{1}, m_{2}, n_{2}, \ldots, n_{k-1}, m_{k} \neq 0$. The length

of this element is

$$
|w|=\left|m_{1}\right|+\left|n_{1}\right|+\cdots+\left|m_{k}\right|+\left|n_{k}\right| .
$$

The map $w \mapsto|w|, \mathbb{F}_{2} \rightarrow \mathbb{Z}^{+}$, is an epimorphism such that the set $\left\{w \in \mathbb{F}_{2}:|w|=n\right\}$ is finite for each $n \in \mathbb{Z}^{+}$. 
EXAmPLE 3.43. Let $G$ be a group with identity $e$, and consider the Rees matrix semigroups

$\mathcal{M}^{o}\left(G, P_{n}, n\right)$ and $\mathcal{M}^{o}\left(G, Q_{n}, n\right)$ in the above notation, where $n \in \mathbb{N}$ and

$$
P_{n}=\left(\begin{array}{cccccc}
e & o & o & \cdots & o & o \\
e & e & o & \cdots & o & o \\
e & e & e & \cdots & o & o \\
\vdots & \vdots & \vdots & \ddots & \vdots & \vdots \\
e & e & e & \cdots & e & o \\
e & e & e & \cdots & e & e
\end{array}\right), \quad Q_{n}=\left(\begin{array}{cccccc}
e & o & o & \cdots & o & o \\
e & e & o & \cdots & o & o \\
o & e & e & \cdots & o & o \\
\vdots & \vdots & \vdots & \ddots & \vdots & \vdots \\
o & o & o & \cdots & e & o \\
o & o & o & \cdots & e & e
\end{array}\right)
$$

We denote these special semigroups by $\mathbf{P}_{n}(G)$ and $\mathbf{Q}_{n}(G)$, respectively. Then we see that

$$
\left|E\left(\mathbf{P}_{n}(G)\right)\right|=\frac{1}{2}\left(n^{2}+n+2\right) \quad \text { and } \quad\left|E\left(\mathbf{Q}_{n}(G)\right)\right|=2 n,
$$

We set $p=(e)_{11}$ and $q=(e)_{12}$, so that $p, q \in E\left(\mathbf{P}_{n}(G)\right), q \neq p$, and $p q=q$ and $q p=p$.

The matrices $P_{n}$ and $Q_{n}$ are regular, so that both $\mathbf{P}_{n}(G)$ and $\mathbf{Q}_{n}(G)$ are regular Rees matrix semigroups with a zero over the group $G$.

ExAmple 3.44. Let $A$ be a Banach algebra, and set $B=\left(A^{\prime \prime}, \square\right)$. Fix $\varphi \in \Phi_{B}$, and let

$$
V_{\varphi}=\left\{\Phi \in A^{\prime \prime}:\|\Phi\|=\varphi(\Phi)=1\right\} .
$$

Then $\left(V_{\varphi}, \square\right)$ is a compact, right topological semigroup (with respect to the $\sigma\left(A^{\prime \prime}, A^{\prime}\right)$-topology), and so the minimum ideal $K\left(V_{\varphi}\right)$ exists. 



\section{CHAPTER 4}

\section{Semigroup algebras}

In this chapter we shall give the definitions and some basic properties of semigroup algebras; we shall conclude with a collection of examples of these algebras. The Banach space $\ell^{1}(S)$ was defined in the introduction.

Basic definitions A semigroup algebra is the 'analytic' version of the standard algebraic semigroup algebra; it is just the Banach algebra generated by the semigroup.

Definition 4.1. Let $S$ be a semigroup, and let $f=\sum \alpha_{r} \delta_{r}$ and $g=\sum \beta_{s} \delta_{s}$ belong to $\ell^{1}(S)$. Set

$$
f \star g=\left(\sum \alpha_{r} \delta_{r}\right) \star\left(\sum \beta_{s} \delta_{s}\right)=\sum\left\{\left(\sum_{r s=t} \alpha_{r} \beta_{s}\right) \delta_{t}: t \in S\right\},
$$

where $\sum_{r s=t} \alpha_{r} \beta_{s}=0$ when there are no elements $r$ and $s$ in $S$ with $r s=t$. Then $\left(\ell^{1}(S), \star\right)$ is the semigroup algebra

$$
\text { of } S \text {. }
$$

Semigroup algebras have been discussed in many papers; see, for example, $[6]$. Important early papers are $[29]$ and $[73]$. Of course, a special case of a semigroup algebra is a group algebra $\ell^{1}(G)$, where $G$ is a group; there is a huge literature on these Banach algebras (see [72] and $[\mathbf{1 9}, \S 3.3]$, for example).

Let $S$ be a semigroup, and set $A=\left(\ell^{1}(S), \star,\|\cdot\|_{1}\right)$. Clearly $A$ is a Banach algebra; it is commutative if and only if $S$ is abelian. Also $A^{\#}=\ell^{1}\left(S^{\#}\right)$. Suppose that $S$ is unital with identity $e_{S}$. Then $\delta_{e_{S}}$ is the identity of $\ell^{1}(S)$, and $\left\|\delta_{e_{S}}\right\|_{1}=1$. However $\ell^{1}(S)$ may have an identity $e_{A}$ in the case where $S$ is non-unital; as we shall see, it may be that $\left\|e_{A}\right\|_{1}>1$ and that $\left\|e_{A}\right\|_{1}$ is not a natural number, although we do not know an example where $\left\|e_{A}\right\|_{1}$ is not a rational number. We shall calculate some values of $\left\|e_{A}\right\|_{1}$ in the case where $A$ is amenable in Examples 10.13-10.15, and in Example 10.15 we shall see that supp $e_{A}$ is not necessarily finite. 
We shall often regard an element $s$ of $S$ as an element of $A$ by the obvious identification; for example, we set

$$
\sigma_{A}(s)=\sigma_{A}\left(\delta_{s}\right) \text { and } \rho_{A}(s)=\rho_{A}\left(\delta_{s}\right) .
$$

Let $T$ be a subset of a semigroup $S$. Then we regard $\ell^{1}(T)$ as a closed, complemented subspace of $A$; if $T$ is a subsemigroup of $S$, then $\ell^{1}(T)$ is a closed subalgebra of $A$; if $T$ is an ideal of $S$, then $\ell^{1}(T)$ is a closed ideal in $A$.

Let $S$ and $T$ be semigroups, and let $\theta: S \rightarrow T$ be an epimorphism. Then there is an induced contractive epimorphism $\theta: \ell^{1}(S) \rightarrow \ell^{1}(T)$ defined by requiring that $\theta \mid S$ takes its specified value in $T \subset \ell^{1}(T)$. Of course, $\ell^{1}(S)$ is isometrically isomorphic to $\ell^{1}(T)$ if and only if $S$ is isomorphic to $T$.

For each $\varphi \in \Phi_{S}$, the map

$$
\sum \alpha_{s} \delta_{s} \mapsto \sum \alpha_{s} \varphi(s)
$$

is a character on $\ell^{1}(S)$, and every character on $\ell^{1}(S)$ arises in this way, and so we identify the character space of the Banach algebra $\ell^{1}(S)$ with the semi-character space $\Phi_{S}$; the specified topology of pointwise convergence on $S$ coincides with the Gel'fand topology. See [35, Proposition 4.1.2].

The character on $\ell^{1}(S)$ that corresponds to the augmentation character on $S$ is the augmentation character

$\varphi_{S}$, where

$$
\varphi_{S}: \sum \alpha_{s} \delta_{s} \mapsto \sum \alpha_{s}
$$

Suppose that $T$ is a subsemigroup of $S$. Then $\varphi_{S} \mid \ell^{1}(T)=\varphi_{T}$.

DeFinition 4.2. Let $S$ be a semigroup, and let $f=\sum_{s \in S} \alpha_{s} \delta_{s}$ belong to $\ell^{1}(S)$. Then

$$
\widehat{f}: \varphi \mapsto \varphi(f)=\sum_{s \in S} \alpha_{s} \varphi(s), \quad \Phi_{S} \rightarrow \mathbb{C},
$$

is the Fourier transform of

$f$, the map $\mathcal{F}: f \mapsto \widehat{f}$ is the Fourier transform, and

$$
A\left(\Phi_{S}\right):=\left\{\widehat{f}: f \in \ell^{1}(S)\right\}
$$

is the algebra of Fourier transforms of $\ell^{1}(S)$.

Thus $\mathcal{F}$ is a homomorphism which can be identified with the Gel'fand transform of $\ell^{1}(S)$, and $A\left(\Phi_{S}\right)$ is a Banach function algebra on the locally compact space $\Phi_{S}$; it is unital when $S$ has an identity.

In the case where $G$ is a group with commutator subgroup $N$, there is an obvious identification of $A\left(\Phi_{G}\right)$ with $A\left(\Phi_{G / N}\right)$, and so $A\left(\Phi_{G}\right)$ is 
a self-adjoint, dense subalgebra of $C\left(\Phi_{G}\right)$, and $A\left(\Phi_{G}\right)$ is regular in the sense of [19, Definition 4.1.16]. However $A\left(\Phi_{S}\right)$ need not be regular for a semigroup $S$. For example, let $S=\mathbb{Z}^{+}$, so that $A\left(\Phi_{S}\right)$ is the Banach function algebra $A^{+}(\overline{\mathbb{D}})$ of absolutely convergent Fourier series on $\overline{\mathbb{D}}$; each function in $A^{+}(\overline{\mathbb{D}})$ is analytic on $\mathbb{D}$.

Let $S$ be an abelian semigroup. Then $\ell^{1}(S)$ is semisimple if and only if $\Phi_{S}$ separates the points of $S$, in the sense that, for each $s, t \in S$ with $s \neq t$, there exists $\varphi \in \Phi_{S}$ such that $\varphi(s) \neq \varphi(t)[35$, Proposition 4.1.4].

\section{Approximate identities}

Proposition 4.3. Let $S$ be a semigroup for which there is a finite subset $F \subset E(S)$ such that

$$
S=\bigcup\{p S q: p, q \in F\} .
$$

Suppose that $\ell^{1}(S)$ has a left approximate identity and a right approximate identity. Then $\ell^{1}(S)$ has an identity.

Proof. Set $A=\ell^{1}(S)$.

There exist $k \in \mathbb{N}, p_{1}, \ldots, p_{k}, \in F$, and pairwise disjoint subsets $T_{i}$ of $S$ for $i \in \mathbb{N}_{k}$ such that $T_{i} \subset p_{i} S\left(i \in \mathbb{N}_{k}\right)$ and $S=\bigcup\left\{T_{i}: i \in \mathbb{N}_{k}\right\}$. For each $f \in A$ and $i \in \mathbb{N}_{k}$, we have $f\left|T_{i}=\left(\delta_{p_{i}} \star f\right)\right| T_{i}$.

Since $A$ has a left approximate identity and $F$ is finite, there is a sequence $\left(f_{n}\right)$ in $A$ such that

$$
\left\|f_{n} \star \delta_{p}-\delta_{p}\right\|_{1}<\frac{1}{n} \quad(n \in \mathbb{N}, p \in F) .
$$

We claim that $\left(f_{n}\right)$ is a Cauchy sequence. Take $\lambda \in\left(A^{\prime}\right)_{[1]}=\ell^{\infty}(S)_{[1]}$, and, for $i \in \mathbb{N}_{k}$, set $\lambda_{i}=\lambda \mid T_{i}$, so that $\lambda_{i} \in\left(A^{\prime}\right)_{[1]}$; clearly, we have $\lambda=\sum_{i=1}^{k} \lambda_{i}$. For $m<n$ and $i \in \mathbb{N}_{k}$, we have

$$
\left|\left\langle f_{m}-f_{n}, \lambda_{i}\right\rangle\right|=\left|\left\langle\delta_{p_{i}} \star\left(f_{m}-f_{n}\right), \lambda_{i}\right\rangle\right| \leq \frac{2}{m},
$$

and so $\left|\left\langle f_{m}-f_{n}, \lambda\right\rangle\right| \leq 2 k^{2} / m$. Thus $\left\|f_{m}-f_{n}\right\|_{1} \leq 2 k^{2} / m$, giving the claim.

Set $f=\lim _{n \rightarrow \infty} f_{n} \in A$, and take $i \in \mathbb{N}_{k}$ and $t \in T_{i}$. Then

$$
f \star \delta_{t}=\lim _{n \rightarrow \infty} f_{n} \star \delta_{p_{i}} \star \delta_{t}=\delta_{p_{i}} \star \delta_{t}=\delta_{t}
$$

by (4.1). Since $S=\bigcup\left\{T_{i}: i \in \mathbb{N}_{k}\right\}$, it follows that $f$ is a left identity of $A$.

Similarly $A$ has a right identity, and has $A$ has an identity. 
We shall note in Example 4.9 that a semigroup algebra $\ell^{1}(S)$ for which $S=E(S)$ can have a bounded approximate identity without having an identity, and so the requirement that the set $F$ be finite cannot be deleted in the above result.

Actions on dual modules Let $S$ be a semigroup. The dual module action of $s \in S$ on the dual space $\ell^{1}(S)^{\prime}=\ell^{\infty}(S)$ is specified by

$$
(s \cdot \lambda)(t)=\lambda(t s), \quad(\lambda \cdot s)(t)=\lambda(s t) \quad(t \in S),
$$

so that $s \cdot \lambda=\lambda \circ R_{s}$ and $\lambda \cdot s=\lambda \circ L_{s}$ for $s \in S$. Note that $|s \cdot \lambda|_{S},|\lambda \cdot s|_{S} \leq|\lambda|_{S}$. For each subset $F$ of $S$, we have $\chi_{F} \in \ell^{\infty}(S)$, and, further,

$$
\chi_{F} \cdot s=\chi_{s^{-1} F}, \quad s \cdot \chi_{F}=\chi_{F s^{-1}} .
$$

It follows that a continous linear operator $D: \ell^{1}(S) \rightarrow \ell^{\infty}(S)$ is a derivation if and only if

$$
\langle r, D(s t)\rangle=\langle t r, D(s)\rangle+\langle r s, D(t)\rangle \quad(r, s, t \in S) ;
$$

such a derivation $D$ is inner if and only if there exists $\lambda \in \ell^{\infty}(S)$ such that

$$
\langle t, D(s)\rangle=\langle t s-s t, \lambda\rangle \quad(s, t \in S) .
$$

Now suppose that $V$ is a compact, right topological semigroup. Then the 'translation maps'

are transfered to the Banach space $M(V)$ as follows. Let $v \in V$ and $\mu \in M(V)$. Then $L_{v} \mu$ and $R_{v} \mu$ are defined by

$$
\left\langle L_{v} \mu, \lambda\right\rangle=\langle\mu, \lambda \cdot v\rangle, \quad\left\langle R_{v} \mu, \lambda\right\rangle=\langle\mu, v \cdot \lambda\rangle \quad(\lambda \in C(V)) .
$$

In the case where $s \in V$ is identified with an element of $M(V)$, the definitions of $L_{v} \delta_{s}$ and $R_{v} \delta_{s}$ coincide with the values of $L_{v}(s)$ and $R_{v}(s)$ given in (3.6). Clearly $L_{v} \mu, R_{v} \mu \in M(V)^{+}$for each $\mu \in M(V)^{+}$.

Proposition 4.4. Let $V$ be a compact, right topological semigroup.

(i) Let $\mu \in M(V)^{+}$and $v \in V$. Then $\left\|R_{v} \mu\right\|=\|\mu\|$.

(ii) Let $B$ be a Borel subset of $V$, and let $\mu \in M(V)$ be such that $|\mu|(V \backslash B)=0$. Let $v \in V$ be such that $R_{v} \mid B$ is injective. Then $\left\|R_{v} \mu\right\|=\|\mu\|$.

(iii) Let $\mu \in M(V)$, and let $v$ be a right cancellable element of $V$. Then $\left\|R_{v} \mu\right\|=\|\mu\|$.

Proof. (i) We have $\left\|R_{v} \mu\right\|=\left\langle R_{v} \mu, 1\right\rangle=\langle\mu, 1 \cdot v\rangle=\langle\mu, 1\rangle=\|\mu\|$.

(ii) Certainly $\left\|R_{v} \nu\right\| \leq\|\nu\|$ for each $v \in V$ and $\nu \in M(V)$.

First suppose that $\mu \in M_{\mathbb{R}}(V)$, and take $\varepsilon>0$. Then there exist $\mu_{1}, \mu_{2} \in M(V)^{+}$and disjoint compact subsets $K_{1}$ and $K_{2}$ of $B$ such that 
$\mu=\mu_{1}-\mu_{2},\|\mu\|=\left\|\mu_{1}\right\|+\left\|\mu_{2}\right\|$, and $\mu_{j}\left(K_{j}\right)>\left\|\mu_{j}\right\|-\varepsilon(j=1,2)$. The sets $R_{v}\left(K_{1}\right)$ and $R_{v}\left(K_{2}\right)$ are disjoint in $V$ because $R_{v} \mid B$ is injective. Thus

$$
\begin{aligned}
\left\|R_{v} \mu\right\| & =\left\|R_{v} \mu_{1}-R_{v} \mu_{2}\right\| \geq\left(R_{v} \mu_{1}\right)\left(R_{v}\left(K_{1}\right)\right)+\left(R_{v} \mu_{2}\right)\left(R_{v}\left(K_{2}\right)\right) \\
& \geq\left\|\mu_{1}\right\|+\left\|\mu_{2}\right\|-2 \varepsilon=\|\mu\|-2 \varepsilon .
\end{aligned}
$$

This holds for each $\varepsilon>0$, and so $\left\|R_{v} \mu\right\|=\|\mu\|$.

Now supppose that $\mu \in M(V)$. Then we have $\mu=\mu_{1}+\mathrm{i} \mu_{2}$, where $\mu_{1}, \mu_{2} \in M_{\mathbb{R}}(V)$. There are four measurable subsets of $V \backslash B$ such that, on each of these subsets, each of $\mu_{1}$ and $\mu_{2}$ is either positive or negative. Since the images of these four sets under the map $R_{v}$ are pairwise disjoint, it is sufficient to prove the result on each of these four sets separately; in fact, we suppose that $\mu_{1}$ and $\mu_{2}$ are both positive.

By a standard theorem [123, Theorem 6.9], there exist $h \in L^{1}\left(\mu_{1}\right)$ and $\nu \in M(V)$ singular to $\mu_{1}$ with

$$
\mu_{2}=\int_{V} h \mathrm{~d} \mu_{1}+\nu .
$$

Set $A=\operatorname{supp} \nu$ and $C=V \backslash A$. It is sufficient to prove the result separately for $\mu \mid A$ and $\mu \mid C$. The result for $\mu \mid A$ is immediate.

Fix $\varepsilon>0$. By replacing $\mu$ by $\mu^{\prime} \in M(V)$ with $\left\|\mu-\mu^{\prime}\right\|<\varepsilon$, we may suppose that $h$ is a simple function. By again partitioning $V$, we may suppose that $h$ is constant, and hence that $\mu_{2} \in \mathbb{C} \mu_{1}$. The result in this case follows immediately from the case where $\mu \in M_{\mathbb{R}}(V)$. We conclude that $\left\|R_{v} \mu\right\|>\|\mu\|-\varepsilon$. This holds for each $\varepsilon>0$, and so the result holds.

(iii) This is an (easier) special case of (ii).

Results related to (iii) are given in [44, Lemma 2] and [103, Corollary p. 469].

The spectrum of an element Let $S$ be a non-unital semigroup, let $s \in S$, and set $A=\ell^{1}(S)$. We shall calculate $\sigma(s)$. Note that always $0 \in \sigma(s)$. There are two cases. First suppose that $\left\{s^{n}: n \in \mathbb{N}\right\}$ is finite, choose the minimum $m \in \mathbb{N}$ such that $s^{m}=s^{n}$ for some $n>m$ (so that $m$ is the index

of $S$ ), and then choose the minimum $k \in \mathbb{N}$ such that $s^{m}=s^{m+k}$ (so that $k$ is the period

of $s$ ). This is the finite case; clearly $\langle s\rangle=\left\{s, s^{2}, \ldots, s^{m+k-1}\right\}$. Second suppose that the elements $s^{n}$ are all distinct for $n \in \mathbb{N}$; this is the infinite case 
Proposition 4.5. Let $S$ be a non-unital semigroup, and let $s \in S$. Set $A=\ell^{1}(S)$. Then:

(i) in the finite case, $\sigma_{A}(s)=\left\{0,1, \zeta, \ldots, \zeta^{k-1}\right\}$, where $k$ is the period of $s$ and $\zeta=\exp (2 \mathrm{i} \pi / k)$;

(ii) in the infinite case, $\sigma_{A}(s)=\overline{\mathbb{D}}$.

Proof. Let $B=\ell^{1}\left(\langle s\rangle^{\#}\right)$, the closed, commutative, unital subalgebra of $\ell^{1}\left(S^{\#}\right)$ generated by $\delta_{s}$. Then $\sigma_{B}(s)=\left\{\varphi(s): \varphi \in \Phi_{B}\right\}$.

(i) Take $m, k \in \mathbb{N}$ as specified in the definition of the finite case. Then $B$ is isomorphic to the finite-dimensional space $\mathbb{C}^{m+k}$ for a certain product on $\mathbb{C}^{m+k}$. Set $\zeta=\varphi(s)$. Then $\zeta^{m}=\zeta^{m+k}$ and so either $\zeta=0$ or $\zeta^{k}=1$. Conversely, given $\zeta$ satisfying these conditions, the map which is defined by $s^{j} \mapsto \zeta^{j}\left(j \in \mathbb{Z}_{m+k-1}^{+}\right)$is a semi-character on $\langle s\rangle^{\#}$, and hence gives a character on $B$. Thus $\sigma_{B}(s)=\left\{0,1, \zeta, \ldots, \zeta^{k-1}\right\}$. In the case where $\zeta^{k}=1$ and $\zeta \neq 1$, we may suppose that $\zeta=\exp (2 \mathrm{i} \pi / k)$. The result follows from equation (1.3).

(ii) Certainly $0 \in \sigma_{A}(s) \subset \overline{\mathbb{D}}$ and $\sigma_{A}(s) \cap \mathbb{T} \neq \emptyset$.

Assume towards a contradiction that there exists $z \in \rho_{A}(s)$ such that $0<|z|<1$. Then there is a subset $J$ of $\mathbb{N}$, a set $\left\{t_{j}: j \in J\right\}$ of distinct points in $S \backslash\langle s\rangle$, and $\left\{\beta_{j}: j \in \mathbb{N}\right\}$ and $\left\{\gamma_{j}: j \in J\right\}$ in $\mathbb{C}$ such that $\sum_{j \in \mathbb{N}}\left|\beta_{j}\right|<\infty, \sum_{j \in J}\left|\gamma_{j}\right|<\infty$, and

$$
z\left(\sum_{j \in \mathbb{N}} \beta_{j} s^{j}+\sum_{j \in J} \gamma_{j} t_{j}\right)=s+\sum_{j \in \mathbb{N}} \beta_{j} s^{j+1}+\sum_{j \in J} \gamma_{j}\left(s t_{j}\right) .
$$

Set $J_{1}=\left\{j \in J: s t_{j}=s\right\}$ and $c_{1}=\sum_{j \in J_{1}} \gamma_{j}$. Then $z \beta_{1}=1+c_{1}$ (equating coefficients of $s$ ). Assume that $c_{1} \neq 0$, so that $J_{1} \neq \emptyset$. Inductively define sets $J_{n}$ by setting $J_{n+1}=\left\{j \in J: s t_{j} \in J_{n}\right\}$. Then $\left(J_{n}\right)$ is a sequence of subsets of $J$. For $n \in \mathbb{N}$, set $c_{n}=\sum_{j \in J_{n}} \gamma_{j}$. Then $z c_{n+1}=c_{n}(n \in \mathbb{N})$, and so $c_{n}=c_{1} / z^{n}$ for each $n \in \mathbb{N}$, and

$$
\sum_{j \in J_{n}}\left|\gamma_{j}\right| \geq\left|c_{1}\right| /|z|^{n}
$$

Thus $\sum_{j \in J}\left|\gamma_{j}\right| \geq\left|c_{1}\right| /|z|^{n}$ for each $n \in \mathbb{N}$, a contradiction unless $c_{1}=0$. Thus $c_{1}=0$ and $z \beta_{1}=1$.

Assume inductively that $z^{n} \beta_{n}=1$ for $n=1, \ldots, k$, and set

$$
L_{1}=\left\{j \in J: s t_{j}=s^{k+1}\right\} \quad \text { and } \quad d_{1}=\sum_{j \in L_{1}} \gamma_{j} .
$$

Then $z \beta_{k+1}=\beta_{k}+d_{1}$. As before, we obtain a contradiction in the case where $d_{1} \neq 0$. Thus $d_{1}=0$ and $z \beta_{k+1}=\beta_{k}$, whence $z^{n} \beta_{n}=1$ for 
$n=k+1$. We conclude by induction that $z^{n} \beta_{n}=1(n \in \mathbb{N})$. But this contradicts the fact that $\sum_{j \in \mathbb{N}}\left|\beta_{j}\right|<\infty$. Thus $\mathbb{D} \subset \sigma(s)$.

It follows that $\sigma_{A}(s)=\overline{\mathbb{D}}$, as required.

Semigroup algebras as dual Banach algebras We determine when a semigroup algebra is a dual Banach algebra.

THEOREM 4.6. Let $S$ be an infinite semigroup. Then the following are equivalent:

(a) $\ell^{1}(S)$ is a dual Banach algebra

with respect to $c_{0}(S)$;

(b) $S$ is

weakly cancellative.

Proof. Set $A=\ell^{1}(S)$.

(b) $\Rightarrow\left(\right.$ a) Let $\lambda \in c_{0}(S)$ and $s \in S$. We claim that $s \cdot \lambda \in c_{0}(S)$. Indeed, take $\varepsilon>0$. There is a finite set $F \subset S$ such that

$$
|\lambda(t)|<\varepsilon \quad(t \in S \backslash F) .
$$

By hypothesis, $F s^{-1}$ is a finite set, and

$$
|(s \cdot \lambda)(r)|<\varepsilon \quad\left(r \in S \backslash F s^{-1}\right) .
$$

Thus the claim holds. Similarly, $\lambda \cdot s \in c_{0}(S)$, and so $c_{0}(S)$ is a submodule of $A^{\prime}$. Since $c_{0}(S)^{\prime}=\ell^{1}(S), A$ is a dual Banach algebra.

(a) $\Rightarrow$ (b) Assume that $S$ is not weakly cancellative, say $S$ is not weakly left cancellative. Then there exist $t, u \in S$ and distinct points $s_{1}, s_{2}, \ldots \in S$ such that $s_{n} t=u(n \in \mathbb{N})$. Let

$$
f_{n}=\frac{1}{n}\left(s_{1}+\cdots+s_{n}\right) \in A \quad(n \in \mathbb{N}) .
$$

Then $\left(f_{n}\right)$ is a sequence in $A$, and clearly $f_{n} \rightarrow 0$ in $\sigma\left(A, c_{0}(S)\right)$. However $f_{n} \star t=u(n \in \mathbb{N})$, and so the sequence $\left(f_{n} \star t\right)$ does not converge to 0 in $\sigma\left(A, c_{0}(S)\right)$. This shows that multiplication in $c_{0}(S)$ is not separately $\sigma\left(A, c_{0}(S)\right)$-continuous, and so $c_{0}(S)$ is not a submodule of $A^{\prime}$, a contradiction of (a).

\section{Examples of semigroup algebras}

ExAmPLE 4.7. Let $D$ be the two-element semigroup specified in Example 3.2. Then the semigroup algebra $\ell^{1}(D)$ is exactly the twodimensional algebra $A_{D}$ of Chapter 2 , page 18 . The amenability constant of this algebra was shown to be 5 . Let $S$ be any semigroup. Then there is an epimorphism $\theta: S^{o} \rightarrow D$ and hence there is a contraction $\theta: \ell^{1}\left(S^{o}\right) \rightarrow \ell^{1}(D)$. 
ExAmple 4.8. Let $S_{0}=\{a, p\}$, and define all products on $S_{0}$ to be $p$. Then $S_{0}$ is a non-unital semigroup, and $A_{0}:=\ell^{1}\left(S_{0}\right)$ is a 2dimensional algebra; the unique character on $A_{0}$ is the augmentation character

$$
\varphi_{0}: \alpha a+\beta p \mapsto \alpha+\beta,
$$

and the radical of $A_{0}$ is the 1-dimensional subspace $R_{0}:=\operatorname{ker} \varphi_{0}$, so that $A_{0}=\mathbb{C} p \oplus R_{0}$ with $A_{0} R_{0}=\{0\}$ and $A_{0}^{2}=\mathbb{C} p$. Thus $A_{0}$ is not semisimple. Here we have $C_{A_{0}}=\{0\} \subsetneq \operatorname{ker} \mathcal{G}$. There is no non-zero point derivation at $\varphi_{0}$, but $A_{0}$ is not weakly amenable because it is not essential.

EXAMPLE 4.9. Let $S$ be the semigroup $\mathbb{N}_{\vee}$, so that the Banach algebra $\ell^{1}(S)$ is commutative. The identity of this algebra is $\delta_{1}$. For $k \in \mathbb{N}$, set $\varphi_{k}(m)=1$ if $m \leq k$ and $\varphi_{k}(m)=0$ if $m>k$. Then $\varphi_{k}$ is a semi-character on $S$, and these semi-characters separate the points of $S$. Thus $\ell^{1}(S)$ is semisimple. Indeed, every semi-character has the form $\varphi_{k}$ for some $k \in \mathbb{N}$, or is the augmentation character; the Gel'fand transform of $\ell^{1}\left(\mathbb{N}_{\vee}\right)$ is the map

$\left(\alpha_{i}: i \in \mathbb{N}\right) \mapsto\left(\left(\sum_{i=1}^{n} \alpha_{i}: n \in \mathbb{N}\right), \sum_{i=1}^{\infty} \alpha_{i}\right) \quad \ell^{1}\left(\mathbb{N}_{\vee}\right) \rightarrow C(\mathbb{N} \cup\{\infty\})$.

For more on this example, see Examples 7.32, 10.10, and 11.3.

EXAMPLE 4.10. Let $S$ be the semigroup $\mathbb{N}_{\wedge}$, so that the Banach algebra $\ell^{1}(S)$ is commutative. For $k \in \mathbb{N}$, set $\psi_{k}(m)=1$ if $m \geq k$ and $\psi_{k}(m)=0$ if $m<k$. Then $\psi_{k}$ is a semi-character on $S$, and these semi-characters separate the points of $S$. Thus $\ell^{1}(S)$ is semisimple. Indeed, every semi-character has the form $\psi_{k}$ for some $k \in \mathbb{N}$, and the Gel'fand transform of $\ell^{1}\left(\mathbb{N}_{\wedge}\right)$ is the map

$$
\left(\alpha_{i}: i \in \mathbb{N}\right) \mapsto\left(\sum_{i=n}^{\infty} \alpha_{i}: n \in \mathbb{N}\right), \quad \ell^{1}\left(\mathbb{N}_{\wedge}\right) \rightarrow c_{0} .
$$

Notice that the sequence $\left(\delta_{n}: n \in \mathbb{N}\right)$ is a bounded approximate identity for the Banach algebra $\ell^{1}\left(\mathbb{N}_{\wedge}\right)$, but that $\ell^{1}\left(\mathbb{N}_{\wedge}\right)$ does not have an identity.

For more on this example, see Examples 7.33, 10.10, and 11.4.

EXAMPLE 4.11. Let $S$ be a set with $|S| \geq 2$, and consider the right zero semigroup

on $S$. Then $f \star g=\varphi_{S}(f) g\left(f, g \in \ell^{1}(S)\right)$. 
Of course, $A:=\ell^{1}(S)$ is the dual space of $E=c_{0}(S)$, but, by Theorem 4.6, $A$ is not a dual Banach algebra with respect to $E$, and so the product in $\ell^{1}(S)$ is not $\sigma(A, E)$-continuous. Note however that $A$ is a dual Banach algebra with respect to $F=c(S)=c_{0}(S) \oplus \mathbb{C} 1$. Indeed, let $f_{\alpha} \rightarrow f$ in $(A, \sigma(A, F))$, and take $g \in A$. For each $\lambda \in F$, we have $\lambda \cdot g=\left(\sum g(s) \lambda(s)\right) 1$, and so

$$
\begin{aligned}
\left\langle g \star f_{\alpha}, \lambda\right\rangle & =\left\langle f_{\alpha}, \lambda \cdot g\right\rangle=\left(\sum g(s) \lambda(s)\right)\left\langle f_{\alpha}, 1\right\rangle \\
& \rightarrow\left(\sum g(s) \lambda(s)\right)\langle f, 1\rangle .
\end{aligned}
$$

Thus $g \star f_{\alpha} \rightarrow g \star f$ in $(A, \sigma(A, F))$. Similarly, $f_{\alpha} \star g \rightarrow f \star g$ in $(A, \sigma(A, F))$. This shows that $A$ is a dual Banach algebra with respect to $F$.

The above is related to Question 2 of D. Blecher [12].

EXAmple 4.12. Let $S$ be a semigroup. We have noted that $\ell^{1}(J)$ is a closed ideal in $\ell^{1}(S)$ for each ideal $J$ in $S$. However the converse is certainly not true. For let $S=\mathbb{Z}^{+}$. Then each ideal in $S$ has the form $J_{n}=\{m \in \mathbb{N}: m \geq n\}$ for $n \in \mathbb{Z}^{+}$, but there are many closed ideals in $\ell^{1}(S) \simeq A\left(\overline{\mathbb{D}}^{+}\right)$which are not of this form; for example, a maximal ideal at $z \in \overline{\mathbb{D}}$ with $z \neq 0$ is not of this form.

Rees semigroup algebras The following example will be of major importance to us.

Let $G$ be a group, let $m, n \in \mathbb{N}$, and consider the Rees matrix semigroup $S$ with a zero over $G$ with a sandwich matrix $P=\left(a_{i j}\right)$, as described in Chapter 3.

Thus

$$
S=\mathcal{M}^{o}(G, P, m, n) .
$$

We now describe the semigroup algebra $\ell^{1}(S)$.

For $x \in G,(x)_{i j}$ is identified with the element of $\mathbb{M}_{m, n}\left(\ell^{1}(G)\right)$ which has $\delta_{x}$ in the $(i, j)^{\text {th. }}$ position and 0 elsewhere, and $o$ is identified with $\delta_{o}$. Thus an element of $\ell^{1}(S)$ is easily identified with an element of $\mathbb{M}_{m, n}\left(\ell^{1}(G)\right) \cup \mathbb{C} \delta_{o}$

We set $N=N(P)$ and $Z=Z(P)$ in the previous notation. Let $f=\left(f_{i j}\right), g=\left(g_{i j}\right) \in \mathbb{M}_{m, n}\left(\ell^{1}(G)\right)$. Then multiplication of $f$ and $g$ in $\ell^{1}(S)$ is given by the formula

$$
\left(f \star_{P} g\right)_{i \ell}=\sum_{(j, k) \in N} f_{i j} \star \delta_{a_{j k}} \star g_{k \ell}+\sum_{(j, k) \in Z} \varphi_{G}\left(f_{i j}\right) \varphi_{G}\left(g_{k \ell}\right) \delta_{o} .
$$

Note that $\mathbb{M}_{m, n}\left(\ell^{1}(G)\right)$ is usually not a subalgebra of $\ell^{1}(S)$. 
We now claim that the quotient Banach algebra $\ell^{1}(S) / \mathbb{C} \delta_{o}$ is isometrically isomorphic to the Munn algebra

$\mathcal{M}\left(\ell^{1}(G), P, m, n\right)$ of Example 2.15. To see this, we must identify the specified sandwich matrix $P \in \mathbb{M}_{n, m}\left(G^{o}\right)$ with a matrix $P \in$ $\mathbb{M}_{n, m}\left(\ell^{1}(G)\right)$ as follows: if the first matrix $P$ has $a \in G$ in the $(i, j)^{\text {th. }}$ position, then the new matrix $P$ has the point mass $\delta_{a}$ in the $(i, j)^{\text {th. }}$. position; if the first matrix $P$ has the element $o$ in the $(i, j)^{\text {th. }}$-position, then the new matrix $P$ has the element $0 \in \ell^{1}(G)$ in the $(i, j)^{\text {th. }}$ position. Thus we can write

$$
\ell^{1}(S) / \mathbb{C} \delta_{o}=\mathcal{M}\left(\ell^{1}(G), P, m, n\right) .
$$

The fact that this identification is an isometry depends on the definition of the norm in $\mathbb{M}_{m, n}(A)$ that was given in equation (1.4).

The product in $\ell^{1}(S)$ also satisfies the following equation:

$$
f \star_{P} \delta_{o}=\delta_{o} \star_{P} f=\left(\sum_{i=1}^{m} \sum_{j=1}^{n} \varphi_{G}\left(f_{i j}\right)\right) \delta_{o}=\varphi_{G}(f) \delta_{o},
$$

say. In particular, take $f, g \in \ell^{1}(G)$ and $i, k \in \mathbb{N}_{m}, j, \ell \in \mathbb{N}_{n}$. Then

$$
(f)_{i j \star_{P}}(g)_{k \ell}=\left\{\begin{array}{cl}
\left(f \star \delta_{a_{j k}} \star g\right)_{i \ell} & \text { if }(j, k) \in N(P), \\
\varphi_{G}(f) \varphi_{G}(g) \delta_{o} & \text { if }(j, k) \in Z(P) .
\end{array}\right.
$$

DEFINITION 4.13. The semigroup algebra corresponding to the semigroup $S=\mathcal{M}^{o}(G, P, m, n)$ is

$$
\ell^{1}(S)=\left(\mathcal{M}^{o}\left(\ell^{1}(G), P, m, n\right), \star_{P}\right) .
$$

In the case where $m=n$, we write $\left(\mathcal{M}^{o}\left(\ell^{1}(G), P, n\right), \star_{P}\right)$; in the case where $P$ is the identity matrix, we write $\left(\mathcal{M}^{o}\left(\ell^{1}(G), m, n\right)\right.$, $)$; in the case where $G=\{e\}$, we write $\left(\mathcal{M}^{o}(\mathbb{C}, P, m, n)\right.$, $\left.\star_{P}\right)$; etc.

This is an elaboration of a standard construction; see [33] and [37]. The case in which $S$ is finite was described earlier in $[\mathbf{1 7}, \S 5.2]$.

We see that we are in the situation of Example 2.11. In that notation, we have $A=\left(\ell^{1}(S), \star_{P},\|\cdot\|_{1}\right)$. Further, $p=\delta_{o} \in \mathfrak{I}(A)$ is such that $\mathbb{C} p$ is an ideal in $A$, and $B=\mathcal{M}\left(\ell^{1}(G), P, m, n\right)$ with the quotient norm; the requirement that

$$
\|a+z p\|_{A}=\|a\|_{B}+|z| \quad(a+z p \in A)
$$

is satisfied. Thus Theorem $2.12(\mathrm{ii})$ shows that $\ell^{1}(S)$ is $(4 C+1)$ amenable whenever $\mathcal{M}\left(\ell^{1}(G), P, m, n\right)$ is $C$-amenable.

The Banach algebra $\ell^{1}(G)$ is unital and has a character, and so Proposition 2.16 applies (where now we have

$$
\left.A=\ell^{1}(G) \text { and } \mathfrak{A}=\mathcal{M}(A, P, m, n)=\ell^{1}(S) / \mathbb{C} \delta_{o} .\right)
$$


Suppose that $\ell^{1}(S)$ has an identity. Then $\mathfrak{A}$ has an identity, and so, as in Proposition 2.16, $m=n, P$ is invertible in $\mathbb{M}_{n}\left(\ell^{1}(G)\right)$, and $\mathfrak{A}$ is topologically isomorphic to $\mathbb{M}_{n}\left(\ell^{1}(G)\right)$. Indeed, the map

$$
\theta: a \rightarrow P^{-1} a, \quad \mathbb{M}_{n}\left(\ell^{1}(G)\right) \rightarrow \mathcal{M}\left(\ell^{1}(G), P, n\right),
$$

is a topological isomorphism. Conversely, suppose that $m=n$ and that $P$ is invertible in $\mathbb{M}_{n}\left(\ell^{1}(G)\right)$, with inverse $Q \in \mathbb{M}_{n}\left(\ell^{1}(G)\right)$, say. Then $\ell^{1}(S) / \mathbb{C} \delta_{o}$ has an identity, and so $\ell^{1}(S)$ has an identity;

it is

$$
e_{A}:=Q+\left(1-\varphi_{G}(Q)\right) \delta_{o}=Q+\left(1-\sum_{i, j=1}^{n} \varphi_{G}\left(Q_{i j}\right)\right) \delta_{o}
$$

as we remarked in Example 2.11.

For $i, j \in \mathbb{N}_{n}$, set $\alpha_{i j}=\varphi_{G}\left(P_{i j}\right)$ and $\beta_{i j}=\varphi_{G}\left(Q_{i j}\right)$, so that $\left(\alpha_{i j}\right),\left(\beta_{i j}\right) \in \mathbb{M}_{n}$, and $\left(\alpha_{i j}\right) \cdot\left(\beta_{i j}\right)=I_{n}$. Since each element of $P$ is either a point mass or 0 , it follows that each $\alpha_{i j}$ is either 0 or 1 ; each $\beta_{i j}$ is clearly a rational number. A necessary condition for $P$ to be invertible is that $\operatorname{det}\left(\alpha_{i j}\right) \neq 0$. Clearly,

$$
\left\|e_{A}\right\|_{1}=\|Q\|+\left|1-\sum_{i, j=1}^{n} \beta_{i j}\right| \geq \sum_{i, j=1}^{n}\left|\beta_{i j}\right|+\left|1-\sum_{i, j=1}^{n} \beta_{i j}\right| .
$$

In the special case where $G=\{e\}$, we identify $P$ with $\left(\alpha_{i j}\right) ; P$ is invertible in $\mathbb{M}_{n}\left(\ell^{1}(G)\right)$ if and only if $\operatorname{det}\left(\alpha_{i j}\right) \neq 0$, and then we have $Q=\left(\beta_{i j} \delta_{e}\right)$, identifed with $\left(\beta_{i j}\right)$. Thus, in this case, we have equality in equation (4.11), and so, curiously, $\left\|e_{A}\right\|_{1}$ is necessarily a rational number; we do not know if this is necessarily the case for general groups $G$.

For each $i \in \mathbb{N}_{n}$, a subset of the set $\left\{\beta_{i 1}, \ldots, \beta_{i n}\right\}$ of numbers sums to 1 ; let the sum of all the numbers $\beta_{i j}$ that do not belong to these designated subsets be $\beta$. Then

$$
\sum_{i, j=1}^{n}\left|\beta_{i j}\right| \geq n+|\beta| \text { and }\left|\sum_{i, j=1}^{n} \beta_{i j}\right|=|n-\beta| \geq n-|\beta|
$$

Thus

$$
\left\|e_{A}\right\|_{1} \geq 2 n-1 \text {. }
$$

For example, take $n \in \mathbb{N}$, and let $P=I_{G}(n)$ be the $n \times n$ identity matrix, as in Chapter 3. Then the identity of $\mathcal{M}^{o}\left(\ell^{1}(G), n\right)$ is

$$
e_{A}=I_{G}(n)-(n-1) \delta_{o} .
$$

Here $|E(S)|=n+1$ (by (3.5)) and $\left\|e_{A}\right\|_{1}=2 n-1$. 
For more on Rees semigroup algebras, see Examples 6.18 and 7.14, and several examples in Chapter 10.

EXAMPLE 4.14. Let $n=2$. Then the three possible forms of regular $2 \times 2$ sandwich matrices over a group $G$ were specified in (3.3). The corresponding matrices in $\mathbb{M}_{2}\left(\ell^{1}(G)\right)$ are

$$
\left(\begin{array}{cc}
\delta_{e} & 0 \\
0 & \delta_{e}
\end{array}\right), \quad\left(\begin{array}{cc}
\delta_{e} & 0 \\
\delta_{e} & \delta_{e}
\end{array}\right), \quad\left(\begin{array}{cc}
\delta_{e} & \delta_{s} \\
\delta_{e} & \delta_{e}
\end{array}\right)
$$

clearly the first two are invertible, with inverses

$$
\left(\begin{array}{cc}
\delta_{e} & 0 \\
0 & \delta_{e}
\end{array}\right) \text { and }\left(\begin{array}{rr}
\delta_{e} & 0 \\
-\delta_{e} & \delta_{e}
\end{array}\right)
$$

respectively, but the third is not invertible in $\mathbb{M}_{2}\left(\ell^{1}(G)\right)$. The identities of the Rees semigroup algebras $\mathcal{M}^{o}\left(\ell^{1}(G), P, 2\right)$ corresponding to these latter two matrices are

$$
\left(\begin{array}{cc}
\delta_{e} & 0 \\
0 & \delta_{e}
\end{array}\right)-\delta_{o} \quad \text { and } \quad\left(\begin{array}{rr}
\delta_{e} & 0 \\
-\delta_{e} & \delta_{e}
\end{array}\right)
$$

both with norm equal to 3 .

EXAMPLE 4.15. Now consider the semigroups $\mathbf{P}_{n}(G)$ and $\mathbf{Q}_{n}(G)$ of Example 3.43. The matrices corresponding to $P_{n}$ and $Q_{n}$ of that example are

$$
P_{n}=\left(\begin{array}{cccccc}
\delta_{e} & 0 & 0 & \cdots & 0 & 0 \\
\delta_{e} & \delta_{e} & 0 & \cdots & 0 & 0 \\
\delta_{e} & \delta_{e} & \delta_{e} & \cdots & 0 & 0 \\
\vdots & \vdots & \vdots & \ddots & \vdots & \vdots \\
\delta_{e} & \delta_{e} & \delta_{e} & \cdots & \delta_{e} & 0 \\
\delta_{e} & \delta_{e} & \delta_{e} & \cdots & \delta_{e} & \delta_{e}
\end{array}\right), Q_{n}=\left(\begin{array}{cccccc}
\delta_{e} & 0 & 0 & \cdots & 0 & 0 \\
\delta_{e} & \delta_{e} & 0 & \cdots & 0 & 0 \\
0 & \delta_{e} & \delta_{e} & \cdots & 0 & 0 \\
\vdots & \vdots & \vdots & \ddots & \vdots & \vdots \\
0 & 0 & 0 & \cdots & \delta_{e} & 0 \\
0 & 0 & 0 & \cdots & \delta_{e} & \delta_{e}
\end{array}\right)
$$

in $\mathbb{M}_{n}\left(\ell^{1}(G)\right)$. In this case, $P_{n}$ and $Q_{n}$ are invertible in $\mathbb{M}_{n}\left(\ell^{1}(G)\right)$, with

$$
P_{n}^{-1}=\left(\begin{array}{cccccc}
\delta_{e} & 0 & 0 & \cdots & 0 & 0 \\
-\delta_{e} & \delta_{e} & 0 & \cdots & 0 & 0 \\
0 & -\delta_{e} & \delta_{e} & \cdots & 0 & 0 \\
\vdots & \vdots & \vdots & \ddots & \vdots & \vdots \\
0 & 0 & 0 & \cdots & \delta_{e} & 0 \\
0 & 0 & 0 & \cdots & -\delta_{e} & \delta_{e}
\end{array}\right)
$$


and

$$
Q_{n}^{-1}=\left(\begin{array}{cccccc}
\delta_{e} & 0 & 0 & \cdots & 0 & 0 \\
-\delta_{e} & \delta_{e} & 0 & \cdots & 0 & 0 \\
\delta_{e} & -\delta_{e} & \delta_{e} & \cdots & 0 & 0 \\
\vdots & \vdots & \vdots & \ddots & \vdots & \vdots \\
\pm \delta_{e} & \mp \delta_{e} & \pm \delta_{e} & \cdots & \delta_{e} & 0 \\
\mp \delta_{e} & \pm \delta_{e} & \mp \delta_{e} & \cdots & -\delta_{e} & \delta_{e}
\end{array}\right)
$$

Thus

$$
\left\|P_{n}^{-1}\right\|=2 n-1 \quad \text { and } \quad \sum_{i, j=1}^{n} \varphi_{G}\left(\left(P_{n}^{-1}\right)_{i j}\right)=1,
$$

so that we have $\left\|e_{\ell^{1}\left(\mathbf{P}_{n}\right)}\right\|_{1}=2 n-1$. Also

$$
\left\|Q_{n}^{-1}\right\|=\frac{1}{2} n(n+1)
$$

and $\sum_{i, j=1}^{n} \varphi_{G}\left(\left(Q_{n}^{-1}\right)_{i j}\right)$ is $n / 2$ for $n$ even and $[n / 2]+1$ for $n$ odd, so that $\left\|e_{\ell^{1}\left(\mathbf{P}_{n}\right)}\right\|_{1} \sim n^{2} / 2$. We see that

$$
\frac{\left\|P_{n}^{-1}\right\|}{\left|E\left(\mathbf{P}_{n}(G)\right)\right|}=\frac{2(2 n-1)}{n^{2}+n+2} \rightarrow 0 \quad \text { as } \quad n \rightarrow \infty,
$$

whereas

$$
\frac{\left\|Q_{n}^{-1}\right\|}{\left|E\left(\mathbf{Q}_{n}(G)\right)\right|} \geq \frac{n(n+1)}{4 n} \rightarrow \infty \quad \text { as } \quad n \rightarrow \infty
$$

We consider when two of our Rees semigroup algebras are isomorphic.

Let $G$ be a group, and let $n \in \mathbb{N}$. As before, all indices belong to the set $\mathbb{N}_{n}$, unless we say otherwise. The matrix units in

$\mathbb{M}_{n}\left(\ell^{1}(G)\right)$ are $E_{i j}=\left(\delta_{e}\right)_{i j}$. Let $W \in \mathbb{M}_{n}\left(\ell^{1}(G)\right)$ be the matrix in which all terms are equal to $\delta_{e}$, so that $E_{i j} W E_{k \ell}=E_{i \ell}$.

Let $P \in \mathbb{M}_{n}\left(G^{o}\right)$ be a sandwich matrix, and take $\left(\alpha_{i j}\right)$ as above, so that each $\alpha_{i j} \in\{0,1\}$. Set $S=\mathcal{M}^{o}(G, P, n)$. Then we identify

$$
\ell^{1}(S)=\left(\mathcal{M}^{o}\left(\ell^{1}(G), P, n\right), \star_{P}\right)
$$

with the linear space $\mathbb{M}_{n}\left(\ell^{1}(G)\right) \oplus \mathbb{C} \delta_{0}$. By equations (4.7) and (4.8), the product $\star_{P}$ is given by:

$$
\left\{\begin{array}{c}
A \star_{P} B=A P B+\varphi_{G}(A(W-P) B) \delta_{0}, \\
A \star_{P} \delta_{o}=\delta_{o} \star_{P} A=\varphi_{G}(A) \delta_{o}, \quad \delta_{o} \star_{P} \delta_{o}=\delta_{o} .
\end{array}\right.
$$

Now suppose that the sandwich matrix $P$ is invertible, so that $\operatorname{det}\left(\alpha_{i j}\right) \neq 0$. 
We set $\beta_{i}=\sum_{r} \beta_{r i}$. As in (4.10), the identity of $\ell^{1}(S)$ is

$$
e_{A}=Q+\left(1-\varphi_{G}(Q)\right) \delta_{o} .
$$

Theorem 4.16. Let $G$ be a group, let $n \in \mathbb{N}$, and let $P$ be an invertible sandwich matrix in $\mathbb{M}_{n}\left(\ell^{1}(G)\right)$, with $Q=P^{-1}$. Then the map

$$
\left\{\begin{aligned}
\theta: A+z \delta_{o} & \mapsto Q A+\varphi_{G}(A-Q A) \delta_{o}+z \delta_{0} \\
\left(\mathcal{M}^{o}\left(\ell^{1}(G), n\right), \star\right) & \rightarrow\left(\mathcal{M}^{o}\left(\ell^{1}(G), P, n\right), \star_{P}\right)
\end{aligned}\right.
$$

is an isomorphism, with inverse

$$
\left\{\begin{aligned}
\theta^{-1}: B+w \delta_{o} & \mapsto P B+\varphi_{G}(B-P B) \delta_{o}+w \delta_{0}, \\
\left(\mathcal{M}^{o}\left(\ell^{1}(G), P, n\right), \star_{P}\right) & \rightarrow\left(\mathcal{M}^{o}\left(\ell^{1}(G), n\right), \star\right) .
\end{aligned}\right.
$$

Proof. Clearly $\theta: \mathcal{M}^{o}\left(\ell^{1}(G), n\right) \rightarrow \mathcal{M}^{o}\left(\ell^{1}(G), P, n\right)$ is a linear map, with inverse $\theta^{-1}$, and so $\theta$ is a bijection.

We must prove that $\theta$ is multiplicative. For this it is sufficient to verify that the relevant coefficients of $\delta_{o}$ are consistent.

As a preliminary, we note that $Q E_{i j}=\sum_{r} \beta_{r i} E_{r j}$, and hence we have $\varphi_{G}\left(Q E_{i j}\right)=\beta_{i}$ and

$$
\theta\left(Q E_{i j}\right)=\sum_{r} \beta_{r i} E_{r j}+\left(1-\beta_{i}\right) \delta_{o} .
$$

Fix $i, j, k, \ell \in \mathbb{N}_{n}$. We shall verify that

$$
\theta\left(E_{i j} \star E_{k \ell}\right)=\theta\left(E_{i j}\right) \star_{P} \theta\left(E_{k \ell}\right) .
$$

First, consider the case where $j \neq k$. Then $E_{i j} \star E_{k \ell}=\delta_{o}$, and so $\theta\left(E_{i j} \star E_{k \ell}\right)=\delta_{o}$. Also

$$
Q E_{i j}(W-P) Q E_{k \ell}=\sum_{r, s} \beta_{r i} \beta_{s k} E_{r \ell}
$$

and so $\varphi_{G}\left(Q E_{i j}(W-P) Q E_{k \ell}\right)=\beta_{i} \beta_{k}$. Hence the coefficient of $\delta_{o}$ in the expression for $\theta\left(E_{i j}\right) \star_{P} \theta\left(E_{k \ell}\right)$ is

$$
\beta_{i} \beta_{k}+\beta_{k}\left(1-\beta_{i}\right)+\beta_{i}\left(1-\beta_{k}\right)+\left(1-\beta_{i}\right)\left(1-\beta_{k}\right)=1 .
$$

This verifies equation (4.16) in the present case.

Second, consider the case where $j=k$. Then $E_{i j} \star E_{j \ell}=E_{i \ell}$, and

$$
\theta\left(E_{i j} \star E_{j \ell}\right)=\sum_{r} \beta_{r i} E_{r \ell}+\left(1-\beta_{i}\right) \delta_{o} .
$$

Also

$$
Q E_{i j}(W-P) Q E_{j \ell}=\sum_{r, s} \beta_{r i} \beta_{s j} E_{s \ell}-\sum_{r} \beta_{r i} E_{r \ell}
$$


and so $\varphi_{G}\left(Q E_{i j}(W-P) Q E_{j \ell}\right)=\beta_{i} \beta_{j}-\beta_{i}$. Hence the coefficient of $\delta_{o}$ in $\theta\left(E_{i j}\right) \star_{P} \theta\left(E_{j \ell}\right)$ is

$$
\beta_{i} \beta_{j}-\beta_{i}+\beta_{i}\left(1-\beta_{j}\right)+\beta_{j}\left(1-\beta_{i}\right)+\left(1-\beta_{i}\right)\left(1-\beta_{j}\right)=1-\beta_{i} .
$$

This verifies equation (4.16) in the present case.

For $A \in \mathbb{M}_{n}\left(\ell^{1}(G)\right)$, we have $\theta\left(A \star \delta_{o}\right)=\varphi_{G}(A) \delta_{o}$ and

$$
\theta(A) \star_{P} \delta_{o}=\left(\varphi_{G}(Q A)+\left(\varphi_{G}(A-Q A)\right) \delta_{o}=\varphi_{G}(A) \delta_{o},\right.
$$

and so again (4.16) holds in this case.

We have verified (4.16) in all cases. 



\section{CHAPTER 5}

\section{Stone-- Čech compactifications}

We recall in this chapter some mostly standard theory of Stone-Čech compactifications of locally compact spaces and, in particular, discrete spaces.

The compactification Let $\Omega$ be a non-empty, locally compact space. We denote by $\beta \Omega$ the Stone-Čech compactification

of $\Omega$. One characterization of $\beta \Omega$ is that it is a compactification of $\Omega$ such that, for each compact space $K$ and each continuous map $f: \Omega \rightarrow K$, there exists a continuous map $\tilde{f}: \beta \Omega \rightarrow K$ such that $\widetilde{f}(x)=f(x) \quad(x \in \Omega)$; a second characterization of $\beta \Omega$ is that it is the character space (or maximal ideal space) of the commutative $C^{*}$ algebra $C B(\Omega)$. See $[\mathbf{1 9}, \S 4.2]$ for details of this correspondence. The classic text on Stone-Cech compactifications is [57].

The closure in $\beta \Omega$ of a subset $U$ of $\Omega$ is denoted by $\bar{U}$, and we set

$$
U^{*}=\bar{U} \cap(\beta \Omega \backslash \Omega),
$$

the growth

of $U$, as in [78], so that $U^{*}$ is a compact topological space. The complements of the sets $\bar{Z}$ for $Z$ a zero set in $\Omega$ are a basis for the closed sets of the topology of $\beta \Omega[\mathbf{1 9}, \S 4.2],[\mathbf{5 7}, \mathrm{p} .87]$.

Let $S$ be a non-empty set. As usual, $\beta S$ is identified with the collection of ultrafilters

on $S$ : for each $u \in \beta S$, the sets of the corresponding ultrafilter are the subsets $U$ of $S$ such that $u \in \bar{U}$, and then $U \in u$.

We shall use the following obvious facts about ultrafilters. Each $\mathcal{A} \subset \mathcal{P}(S)$ such that $\mathcal{A}$ has the finite intersection property is contained in an ultrafilter on $S$. Let $\mathcal{U}$ be an ultrafilter on $S$. Each subset $T$ of $S$ such that $T \cap U \neq \emptyset(U \in \mathcal{U})$ is in $\mathcal{U}$. Suppose that $T_{1}, T_{2} \subset S$ with $T_{1} \cup T_{2} \in \mathcal{U}$. Then $T_{1} \in \mathcal{U}$ or $T_{2} \in \mathcal{U}$. Suppose that $S$ has cardinality $|S|=\kappa$. Then $|\beta S|=2^{2^{\kappa}}$. The standard reference on ultrafilters (for those with good eyesight) is [18].

Let $S$ be a non-empty set. As before, the space $\ell^{\infty}(S)$ is a Banach space with respect to the uniform norm $|\cdot|_{S}$ on $S$, and it is 
a commutative $C^{*}$-algebra for the pointwise product; $c_{0}(S)$ is a $C^{*}$ subalgebra of $\ell^{\infty}(S)$. The space $\beta S$ is homeomorphic to the character space of $\left(\ell^{\infty}(S),|\cdot|_{S}\right)$, and $\left(\ell^{\infty}(S),|\cdot|_{S}\right)$ is isometrically isomorphic to $\left(C(\beta S),|\cdot|_{\beta S}\right)$; we shall identify these two Banach algebras. The space $\beta S$ is extremely disconnected, and $\overline{F \cap G}=\bar{F} \cap \bar{G}$ whenever $F$ and $G$ are subsets of $S[\mathbf{1 9}$, Proposition 4.2.8]. Let $T$ be a subset of $S$. Then we identify $\beta T$ with $\bar{T}$.

The dual Banach space of $\ell^{\infty}(S)=C(\beta S)$ is $M(\beta S)$, and

$$
\left\langle\mu, \chi_{F}\right\rangle=\mu(\bar{F})=\int_{\bar{F}} \mathrm{~d} \mu \quad(\mu \in M(\beta S))
$$

for each subset $F$ of $S$.

The following property of $\beta S$ is well-known; for example, see [78, Theorem 3.36].

Proposition 5.1. Let $S$ be an infinite set. Then each non-empty $G_{\delta}$-set in $S^{*}$ has a non-empty interior in $S^{*}$.

We shall use the following trivial remark about $\beta S$.

Proposition 5.2. Let $S$ be a non-empty set, let $T$ be a subset of $S$, and let $f: \beta S \rightarrow \beta S$ be a continuous function with $f(S) \subset S$. Then $f^{-1}(\bar{T})=\overline{f^{-1}(T)}$.

Proof. Set $R=S \backslash T$, so that $\bar{R}=\beta S \backslash \bar{T}$. Then $\overline{f^{-1}(T)} \subset f^{-1}(\bar{T})$ and $\overline{f^{-1}(R)} \subset f^{-1}(\bar{R})$. Also $\overline{f^{-1}(T)} \cap \overline{f^{-1}(R)}=\emptyset$ because $T \cap R=\emptyset$, and $\overline{f^{-1}(T)} \cup \overline{f^{-1}(R)}=\beta S$, and so $\beta S \backslash \overline{f^{-1}(T)} \subset \beta S \backslash f^{-1}(\bar{T})$.

Let $S_{1}$ and $S_{2}$ be non-empty sets. Then there are two projections

$$
\pi_{j}:\left(s_{1}, s_{2}\right) \mapsto s_{j}, \quad S_{1} \times S_{2} \rightarrow S_{j},
$$

for $j=1,2$. These two maps have continuous extensions to maps $\pi_{j}: \beta\left(S_{1} \times S_{2}\right) \rightarrow \beta S_{j}$, and so there is a continuous surjection

$$
\pi: u \mapsto\left(\pi_{1}(u), \pi_{2}(u)\right), \quad \beta\left(S_{1} \times S_{2}\right) \rightarrow \beta S_{1} \times \beta S_{2} .
$$

This map is a homeomorphism if and only if at least one of the two sets $S_{1}$ and $S_{2}$ is finite.

Uniform ultrafilters The following definition is given in [18, $§ 7$ and [78, Definition 3.13].

Definition 5.3. Let $S$ be an infinite set, and let $\kappa$ be an infinite cardinal with $\kappa \leq|S|$. An ultrafilter $\mathcal{U}$ on $S$ is $\kappa$-uniform if $|U| \geq \kappa$ for each $U \in \mathcal{U}$, and uniform if $|U|=|S|$ for each $U \in \mathcal{U}$. 
We denote the set of points in $S^{*}$ which correspond to $\kappa$-uniform ultrafilters on $S$ by $U_{\kappa}(S)$ and the set of uniform ultrafilters by $U_{S}$. Clearly each $U_{\kappa}(S)$ is a compact subspace of $\beta S$. By [78, Theorem 3.58], $\left|U_{S}\right|=2^{2^{\kappa}}$, where $\kappa=|S|$, and so $U_{S}$ is a non-empty, compact subset of $S^{*}$. Let $T$ be a subset of $S$. If $|T|=|S|$, then the set $T^{*} \cap U_{S}$ is a non-empty, compact subset of $U_{S}$; if $|T|<|S|$, then $T^{*} \cap U_{S}=\emptyset$.

We shall several times use the following easy remark. Let $S$ be an infinite set, and let $\kappa$ be an infinite cardinal with $\kappa \leq|S|$. Let $\mu \in M(\beta S)$ with $\mu \mid U_{\kappa}(S)=0$, and take $\varepsilon>0$. Since $|\mu|$ is a regular Borel measure, there is a compact subset $K$ of $\beta S \backslash U_{\kappa}(S)$ such that $|\mu|(K)>\|\mu\|-\varepsilon$. For each $x \in K$, there exists $U_{x} \in x$ with $\left|U_{x}\right|<\kappa$. There is a finite open cover $\left\{\bar{U}_{x_{1}}, \ldots, \bar{U}_{x_{n}}\right\}$ of $K$, and then $U:=U_{x_{1}} \cup \cdots \cup U_{x_{n}}$ is a subset of $S$ such that $|U|<\kappa$ and $|\mu|(\bar{U})>\|\mu\|-\varepsilon$, so that

$$
\|\mu-\mu \mid \bar{U}\|<\varepsilon .
$$

We shall also require the following result; the present proof was pointed out to us by Andreas Blass, and we are grateful for this.

Proposition 5.4. Let $\kappa$ be an infinite cardinal. Then there is a uniform ultrafilter $\mathcal{U}$ on $\kappa$ such that, for each infinite cardinal $\zeta$ with $\zeta<\kappa$, the set $\kappa$ can be partitioned into $\zeta$ pairwise disjoint sets with the property that, for each cardinal $\eta$ with $\eta<\zeta$, the union of each collection of $\eta$ sets of the partition is not a member of $\mathcal{U}$.

Proof. Set $K=\bigoplus\{\zeta: \zeta<\kappa\}$, and let $p_{\zeta}: K \rightarrow \zeta$ be the appropriate projection. Then $|K|=\kappa$, and so we can identify $K$ and $\kappa$ as sets.

Consider the family $\mathcal{F}$ of all subsets of $K$ of the form $p_{\zeta}^{-1}(S)$, where $S$ is a subset of $\zeta$ such that $|\zeta \backslash S|<\zeta$, and where $\zeta<\kappa$ is an infinite cardinal. It is easy to see that $\mathcal{F}$ has the finite intersection property, and so $\mathcal{F}$ is contained in an ultrafilter, say $\mathcal{U}$, on $K$.

Take an infinite cardinal $\zeta$ with $\zeta<\kappa$. Then

$$
\left\{p_{\zeta}^{-1}(\{\alpha\}) \cap K: \alpha \in \zeta\right\}
$$

is a partition of $K$. For each $\eta<\zeta$, the union $U$ of $\eta$ sets of the partition is the set of elements $\gamma \in K$ such that $p_{\zeta}(\gamma) \in T$ for a certain subset $T$ of $\zeta$ with $|T|=\eta$. The complement of $U$ belongs to $\mathcal{U}$, and so $U \notin \mathcal{U}$, as required.

$P$-points We recall that a point $x$ of a topological space $X$ is a $P$-point of $X$ if each $G_{\delta}$-set in $X$ containing $x$ is a neighbourhood of $x$. 
TheOrem 5.5. (CH) Let $S$ be an infinite, countable set. Then there is a P-element in $S^{*}$.

Proof. This is [18, Theorem 9.13].

By a theorem of Shelah (see $[\mathbf{1 3 0}],[\mathbf{1 3 4}])$ the existence of $P$-points in the space $\mathbb{N}^{*}$ is independent of the theory ZFC. Conditions on an ultrafilter that are equivalent to the corresponding point in $\beta \mathbb{N}$ being a $P$-point of $\mathbb{N}^{*}$ are given in [22, Theorem 2.24]. 


\section{CHAPTER 6}

\section{The semigroup $(\beta S, \square)$}

Let $S$ be a semigroup. We shall show that there are two products and $\diamond$ on the Stone-Cech compactification $\beta S$ of $S$ such that $(\beta S, \square)$ and $(\beta S, \diamond)$ are semigroups, and we shall begin a study of these semigroups. The particular purpose of the present chapter is to establish properties of the semigroups that are to be used in our main study, to be given in Chapters 7 and 11-13, of the Banach algebras $\left(\ell^{1}(S)^{\prime \prime}, \square\right)$ and $\left(\ell^{1}(S)^{\prime \prime}, \diamond\right)$. Our belief is that these properties of the semigroups cast a bright light on those of the Banach algebras defined thereon.

Of course, the semigroups $(\beta S, \square)$ and $(\beta S, \diamond)$ are very well-known; they are the topic of the monograph [78].

The definitions and basic properties Let $S$ be a non-empty set, and let $*$ be a binary operation on $S$, so that $*$ is a map from $S \times S$ to $S$; the image of $(s, t)$ is denoted by $s * t$. For each $s \in S$, the map

$$
L_{s}: t \mapsto s * t, \quad S \rightarrow S \subset \beta S,
$$

has a continuous extension to a map $L_{s}: \beta S \rightarrow \beta S$. For each $u \in \beta S$, define $s \square u=L_{s}(u)$. Next, the map

$$
R_{u}: s \mapsto s \square u, \quad S \rightarrow \beta S,
$$

has a continuous extension to a map $R_{u}: \beta S \rightarrow \beta S$. For $u, v \in \beta S$, set

$$
u \square v=R_{v}(u) .
$$

Then $\square$ is a binary operation on $\beta S$, and the restriction of $\square$ to $S \times S$ is the original binary operation $*$. Further, for each $v \in \beta S$, the map $R_{v}$ is continuous, and, for each $s \in S$, the map $L_{s}$ is continuous.

We see that

$$
u \square v=\lim _{\alpha} \lim _{\beta} s_{\alpha} * t_{\beta}
$$

whenever $\left(s_{\alpha}\right)$ and $\left(t_{\beta}\right)$ are nets in $S$ with $\lim _{\alpha} s_{\alpha}=u$ and $\lim _{\beta} t_{\beta}=v$.

Similarly, we can define a binary operation $\diamond$ on $\beta S$ such that

$$
u \diamond v=\lim _{\beta} \lim _{\alpha} s_{\alpha} * t_{\beta}
$$

whenever $\left(s_{\alpha}\right)$ and $\left(t_{\beta}\right)$ are nets in $S$ with $\lim _{\alpha} s_{\alpha}=u$ and $\lim _{\beta} t_{\beta}=v$. 
Let $(S, \cdot)$ be a semigroup. Then the two extensions of $\cdot$ are the binary operations $\square$ and $\diamond$ on $\beta S$; it is immediately checked that both $\square$ and $\diamond$ are associative on $\beta S$ [78, Theorem 4.4]. Thus we obtain the following fundamental result.

Theorem 6.1. Let $S$ be a semigroup. Then $(\beta S, \square)$ and $(\beta S, \diamond)$ are semigroups containing $S$ as a subsemigroup. Further:

(i) for each $v \in \beta S$, the map $R_{v}: u \mapsto u \square v$ is continuous, and $(\beta S, \square)$ is a compact,

right topological semigroup;

(ii) for each $s \in S$, the map $L_{s}: u \mapsto s \square u$ is continuous;

(iii) the minimum ideal $K(\beta S, \square)$ exists, and $E(\beta S) \neq \emptyset$.

The semigroup maps $\square$ and $\diamond$ that we have defined here agree with the semigroup maps defined in several different ways in [78].

From now on, we shall generally discuss the semigroup $(\beta S, \square)$; of course, analogous results hold for the compact, left topological semigroup $(\beta S, \diamond)$. Let $u, v \in \beta S$. We shall sometimes write $u v$ for $u \square v$ (and $u^{2}$ for $u \square u$, etc.); for subsets $U$ and $V$ of $\beta S$, we set

$$
U \square V=\{u \square v: u \in U, v \in V\} ;
$$

the notations $S_{[n]}^{*}$ and $S_{[\infty]}^{*}$ always refer to subsets of $(\beta S, \square)$, so that, for example,

$$
S_{[n]}^{*}=\left\{u_{1} \square \cdots \square u_{n}: u_{1}, \ldots, u_{n} \in S^{*}\right\} .
$$

Note that, in the case where $S=\mathbb{N}$ and $u, v \in \beta \mathbb{N}$, the element $u \square v$ is denoted by $u+v$ in [78], but, for us, $u+v$ denotes the element $\delta_{u}+\delta_{v} \in \ell^{1}(\beta S)$.

We note that there appear to be several deep open questions about the semigroup $(\beta \mathbb{Z}, \square)$; for example, it is open whether or not there is an element $u \in \beta \mathbb{N}$ with $u^{3}=u^{2}$, but with $u^{2} \neq u$.

We shall see later (in Theorem 12.20) that, in the case where $S$ is a weakly cancellative semigroup, the map $L_{u}$, defined for $u \in \beta S$, is continuous if and only if $u \in S$. However this is not true for every semigroup, as the following example shows.

EXAMPLE 6.2. Let $S$ be a right zero semigroup,

as in Example 3.30. Clearly $u \square v=v(u, v \in \beta S)$, and so $(\beta S, \square)$ is also a right zero semigroup. In this case, $L_{u}$ is continuous for each $u \in \beta S$. 
EXAmple 6.3. Set $S=\mathbb{N}_{\vee}$, a weakly cancellative semigroup. Then $u \square v=v$ and $u \diamond v=u$ for $u, v \in S^{*}$. Thus $\left(S^{*}, \square\right)$ is a right zero semigroup

with $S_{[2]}^{*}=S^{*}$, and $\left(S^{*}, \diamond\right)$ is a left zero semigroup.

Let $S$ be a semigroup without an identity. Then $\beta S$ does not have an identity [78, Corollary 9.30], and $(\beta S)^{\#}=\beta\left(S^{\#}\right)$.

Let $S$ be a semigroup, and let $T$ be a subsemigroup of $S$. We have identified $\beta T$ with $\bar{T}$; in fact, $(\beta T, \square)$ is the subsemigroup $(\bar{T}, \square)$ of $(\beta S, \square)[\mathbf{7 8}$, Remark 4.19]. Suppose that $T$ is a left (respectively, right) ideal in $S$. Then $\beta T$ is a left (respectively, right) ideal in $(\beta S, \square)$.

Let $S$ and $T$ be semigroups, and let $\theta: S \rightarrow T$ be a homomorphism. Then $\theta$ extends to a continuous map $\theta: \beta S \rightarrow \beta T$, and

$$
\theta:(\beta S, \square) \rightarrow(\beta T, \square) \text { and } \theta:(\beta S, \diamond) \rightarrow(\beta T, \diamond)
$$

are homomorphisms.

In fact $(\beta S, \square)$ is the largest compactification of $S$ which is a compact, right topological semigroup, in the sense that any other such compactification is a continuous homomorphic image of $(\beta S, \square)$.

Since $V=(\beta S, \square)$ is a compact, right topological semigroup, we have definitions of $L_{v} \mu$ and $R_{v} \mu$ for each $v \in V$ and $\mu \in M(V)$; clearly, $L_{v} \mu=v \square \mu$ and $R_{v} \mu=\mu \square v$.

Let $S$ be a subsemigroup of a group $G$, and let $s \in S$, with inverse $s^{-1} \in G$. Then $s^{-1} \square \beta S \subset \beta G$; in the case where $S \backslash s S$ is finite, we have $s^{-1} \square S^{*} \subset S^{*}$. Now suppose that $S \backslash s S$ is finite for each $s \in S$. Then $G \square S^{*} \subset S^{*}$. For example, we have $\mathbb{Z} \square \mathbb{N}^{*} \subset \mathbb{N}^{*}$.

The points of $\beta S$ have been identified with ultrafilters on $S$, and so the product $\square$ can be defined in these terms;

the following characterization of the product $u \square v$ is taken from $[\mathbf{7 8}$, Theorem 4.15].

Proposition 6.4. Let $S$ be a semigroup, let $u, v \in \beta S$, and let $W$ be a subset of $S$. Then the following conditions on $W$ are equivalent:

(a) $W$ belongs to the ultrafilter $u \square v$;

(b) $\left\{s \in S: s^{-1} W \in v\right\}$ belongs to $u$;

(c) there exists a set $U \in u$ and a family $\left\{V_{s}: s \in U\right\}$ of sets in $v$ such that $s V_{s} \subset W(s \in U)$.

Let $u, v \in \beta S$, and let $U \in u$ and $V \in v$. Then $u \in \bar{U}$ and $v \in \bar{V}$, and so $u \square v \in \overline{U V}$. Hence $U V \in u \square v$. 
Proposition 6.5. Let $S$ be a cancellative semigroup.

(i) For each $v \in \beta S$, the maps $L_{v} \mid S$ and $R_{v} \mid S$ are injective.

(ii) For each $s \in S$, the maps $L_{s}$ and $R_{s}$ are injective on $\beta S$.

Proof. These are [78, Lemmata 6.28 and 8.1], respectively.

Proposition 6.6. Let $S$ be a semigroup such that $(\beta S, \square)$ is regular. Then $S$ is regular.

Proof. Let $s \in S$. There exists $u \in \beta S$ with $s \square u \square s=s$. Let $\left(t_{\alpha}\right)$ be a net in $S$ such that $\lim _{\alpha} t_{\alpha}=u$. Then $\lim _{\alpha} s t_{\alpha} s=s$, and so $s t_{\alpha} s=s$ eventually. Thus there exists $t \in S$ with $s t s=s$.

Let $S$ be a semigroup. Clearly we have $E\left(S^{*}\right) \subset S_{[\infty]}^{*}$. Note that, for $S$ the semigroup of Example 6.3, we have $S^{*}=S_{[2]}^{*}=S_{[\infty]}^{*}=E\left(S^{*}\right)$.

Let $S=\mathbb{S}_{2}$ or $S=\mathbb{F}_{2}$. Then it is proved in [13] and [1], respectively, that $K(\beta S, \square) \cap K(\beta S, \diamond)=\emptyset$.

Let $S$ be a semigroup, and consider $(E(\beta S), \leq)$, a partially ordered set. In the case where $S$ is weakly left cancellative, no element $p \in S^{*}$ can be a right identity for $\beta S$, and so no element of $E(\beta S)$ is right cancellable. In the case where $S$ is cancellative, $E(\beta S)$ contains an infinite decreasing sequence (combine Theorems 6.12 and 6.32 of $[\mathbf{7 8}]$ ). It is stated in [78] that 'it is a difficult open question' whether or not there exists an increasing infinite chain $p_{1} \leq p_{2} \leq \cdots$ in $E\left(\mathbb{N}^{*}\right)$ with $p_{i} \neq p_{j}$ for $i \neq j$. It follows by combining Theorems 12.29 and 12.45 of [78], that, with $\mathrm{CH}$, there exist maximal elements in $\left(E\left(\mathbb{N}^{*}\right), \leq\right)$; it is not known whether or not this is a theorem of ZFC. See also [?].

We shall require the following result, taken from $[\mathbf{7 8}$, Theorems 9.4 and 9.7 and Lemma 9.5].

Proposition 6.7. (i) Let $G$ be a countable group, and let $q$ be a right maximal idempotent

in $G^{*}$. Set

$$
C=\left\{u \in G^{*}: u \square q=q\right\} .
$$

Then $C$ is a finite, right zero subsemigroup of $\beta G$. Further, suppose that $u, v \in \beta G \backslash(\beta G \square C)$ and that $u \square q=v \square q$. Then $u=v$.

(ii) Let $S$ be a countable, cancellative semigroup. Then each nonempty, open subset of $S^{*}$ which contains an

idempotent also contains a right maximal idempotent.

Let $S$ be a semigroup. There is a discussion of identities in $\beta S$ and $S^{*}$ in $[\mathbf{7 8}, \S 9.4]$. Let us quote [78, Corollary 9.35]. Suppose that $S$ is 
countable and that $\left(S^{*}, \square\right)$ has a right identity. Then $S^{*}$ has at least $2^{\mathfrak{c}}$ right identities, and so $S^{*}$ does not have an identity.

We have results from [78, Theorem 5.8 and Lemma 5.11] about idempotents and finite products that we shall require.

Proposition 6.8. Let $S$ be a semigroup.

(i) Let $\left(s_{n}\right)$ be a sequence in $S$. Then there exists $p \in E(\beta S)$ with

$$
p \in \overline{F P\left\langle\left(s_{n}: n \geq m\right)\right\rangle} \quad(m \in \mathbb{N}) .
$$

(ii) Let $p \in E(\beta S)$ and $A \in p$. Then there exists a sequence $\left(s_{n}\right)$ in $S$ with $F P\left\langle\left(s_{n}\right)\right\rangle \subset A$.

Definition 6.9. Let $S$ be a semigroup, let $T$ be a subset of $S$, and let $u \in \beta S$. Then $T^{\star}(u)=\left\{s \in T: s^{-1} T \in u\right\}$.

Proposition 6.10. Let $S$ be a semigroup, let $T$ be a subset of $S$, and let $u \in \beta S$. Then:

(i) $u \in E(\beta S)$ if and only if $T^{\star}(u) \in u$ whenever $T \in u$;

(ii) $s^{-1} T^{\star}(u) \in u\left(s \in T^{\star}(u)\right)$ whenever $u \in E(\beta S)$.

Proof. The two clauses follow from Proposition 6.4 and from [78, Lemma 4.14], respectively.

Topological centres of the semigroup $\beta S$ We now consider the analogues for semigroups of the topological centres for second duals of Banach algebras that were defined in Definition 2.24. The following concept is well-known; see [98], for example.

Definition 6.11. Let $S$ be a semigroup. The left and right topological centres, $\mathfrak{Z}_{t}^{(\ell)}(\beta S)$ and $\mathfrak{Z}_{t}^{(r)}(\beta S)$, of $\beta S$ are

$$
\mathfrak{Z}_{t}^{(\ell)}(\beta S)=\{u \in \beta S: u \square v=u \diamond v(v \in \beta S)\}
$$

and

$$
\mathfrak{Z}_{t}^{(r)}(\beta S)=\{u \in \beta S: v \square u=v \diamond u(v \in \beta S)\},
$$

respectively; $S$ is Arens regular if $\mathfrak{Z}_{t}^{(\ell)}(\beta S)=\mathfrak{Z}_{t}^{(r)}(\beta S)=\beta S$, left strongly Arens irregular if $\left.\mathfrak{Z}_{t}^{(\ell)} \beta S\right)=S$, right strongly Arens irregular if $\mathfrak{Z}_{t}^{(r)}(\beta S)=S$, and strongly Arens irregular if it is both left and right strongly Arens irregular. 
Our set $\mathfrak{Z}_{t}^{(\ell)}(\beta S)$ is equal to the topological centre

$$
\Lambda(\beta S)=\left\{s \in \beta S: L_{s} \text { is continuous on } \beta S\right\},
$$

as defined in [10] and [78, Definition 2.4].

Let $T$ be a subsemigroup of a semigroup $S$. Clearly

$$
\mathfrak{Z}_{t}^{(\ell)}(\beta S) \cap \beta T \subset \mathfrak{Z}_{t}^{(\ell)}(\beta T) \text { and } \mathfrak{Z}_{t}^{(r)}(\beta S) \cap \beta T \subset \mathfrak{Z}_{t}^{(r)}(\beta T) ;
$$

if $S$ is Arens regular, then so is $T$. We see that $\mathfrak{Z}_{t}^{(\ell)}(\beta S)=\mathfrak{Z}_{t}^{(r)}(\beta T)$ where $T=S^{\text {op }}$.

In Chapter 12, we shall give examples of semigroups which are Arens regular and which are strongly Arens irregular; for example, by Theorem 12.20, each infinite group is strongly Arens irregular. Assuming this, we exhibit here a semigroup which is right, but not left, strongly Arens irregular.

ExAmple 6.12. Let $G$ be an infinite group, and set $S=G \times G$, with the product specified by the formula

$$
(a, x) \cdot(b, y)=(a b, a y) \quad(a, b, x, y \in G),
$$

as in Example 3.35:

We claim that $S$ is not left strongly Arens irregular.

Indeed, take $u \in G^{*}$, say $u=\lim _{\alpha} x_{\alpha}$, where $\left(x_{\alpha}\right)$ is a net in $G$. Then $\left(\left(e_{S}, x_{\alpha}\right)\right)$ is a net in $S$, and it is convergent to an element of $\beta S$ that we may denote by $\left(e_{S}, u\right)$. Since $\left(e_{S}, x_{\alpha}\right) \cdot(b, y)=(b, y)$ for each $\alpha$ and each $(b, y) \in S$, we see that $\left(e_{S}, u\right) \in \mathfrak{Z}_{t}^{(\ell)}(\beta S)$. Since $\left(e_{S}, u\right) \notin S$, the claim holds.

We also claim that $S$ is right strongly Arens irregular. For this we use the continuous surjection $\pi: \beta(G \times G) \rightarrow \beta G \times \beta G$ of equation (5.1). Take $Q \in \mathfrak{Z}_{t}^{(r)}(\beta S)$. For each $u \in \beta G$, we have $\left(u, e_{S}\right) \in \beta S$, and so $\left(u, e_{S}\right) \square Q=\left(u, e_{S}\right) \diamond Q$. Thus $u \square \pi_{1}(Q)=u \diamond \pi_{1}(Q)$ and $u \square \pi_{2}(Q)=u \diamond \pi_{2}(Q)$. It follows that $\pi_{1}(Q), \pi_{2}(Q) \in \mathfrak{Z}_{t}^{(r)}(\beta G)=G$, and so $Q \in G \times G=S$. Thus $\mathfrak{Z}_{t}^{(\ell)}(\beta S)=S$, as claimed.

Subsemigroups and ideals in $\beta S$ Let $S$ be a semigroup. We now consider some subsets, subsemigroups, and ideals in $\beta S$, where $\beta S$ is taken with the semigroup operation $\square$.

Let $S$ be a semigroup. A condition for $S^{*}$ to be a subsemigroup of $\beta S$ is given in [78, Theorem 4.28]. Thus $\left(S^{*}, \square\right)$ is itself a semigroup if and only if, for each $A \in \mathcal{P}_{f}(S)$ and each infinite $B \subset S$, there is a finite subset $F \in \mathcal{P}_{f}(B)$ such that $\bigcap\left\{s^{-1} A: s \in F\right\}$ is finite. Let $S$ be an infinite, trivial semigroup at $p \in S$. Then $S$ does not satisfy 
this condition, and so $S^{*}$ is not a semigroup. In the case where $S^{*}$ is a semigroup, it is a compact, right topological semigroup, and so $E\left(S^{*}\right) \neq \emptyset$, and each of the subsets $S_{[n]}^{*}$ and $S_{[\infty]}^{*}$ is a subsemigroup.

We now consider ideals in $(\beta S, \square)$. For example, we see immediately from (6.1) that $\mathbb{Z}^{+*}$ and $\mathbb{Z}^{-*}$ are closed left ideals in $(\beta \mathbb{Z}, \square)$; of course, neither is a right ideal. We start with the following well-known triviality; it will be used implicitly many times.

Proposition 6.13. Let $S$ be a semigroup, and let $u \in \beta S$. Then the left ideal $\beta S \square u$ in $\beta S$ is closed, and $\beta S \square u=\overline{S \square u}$.

We shall see below that, in general, the right ideal $u \square \beta S$ is not closed in $\beta S$.

Proposition 6.14. Let $S$ be a semigroup.

(i) Let $L$ be a left ideal in $(\beta S, \square)$. Then $\bar{L}$ is also a left ideal.

(ii) Let $I$ be an ideal in $(\beta S, \square)$. Then $\bar{I}$ is also an ideal.

Proof. (i) This is [78, Theorems 2.17].

(ii) This follows from (i) and Theorem 3.25(ii).

Proposition 6.15. (i) Let $S$ be a semigroup. Then $\overline{K(\beta S)}$ is an ideal in $(\beta S, \square)$.

(ii) Let $S$ be an abelian semigroup. Then $\bar{R}$ is an ideal in $(\beta S, \square)$ for each right ideal $R$ in $\beta S$, and $\bar{R}=\overline{K(\beta S)}$ for each minimal right ideal $R$ in $\beta S$.

(iii) The set $\overline{K(\beta \mathbb{N})}$ contains idempotents which are right maximal in $E\left(\mathbb{Z}^{*}\right)$.

Proof. These follow from Theorems 4.44, 2.19(a), and 8.65, respectively, of [78].

Let $p$ be a minimal idempotent in $\beta \mathbb{N}$. Then $p \square \beta \mathbb{N}$ is a minimal right ideal in $\beta \mathbb{N}$, and $\overline{p \square \beta \mathbb{N}}=\overline{K(\beta \mathbb{N})}$, and so the right ideal $p \square \beta \mathbb{N}$ is not closed. It is interesting that it is an open question whether or not $K(\beta S)$ is a prime ideal in $\beta S$; see [77].

The next theorem is [78, Theorems 4.31 and 4.36]; the related result $[\mathbf{3 1}$, Theorem $9(\mathrm{ii})]$ is not correct.

TheOREM 6.16. Let $S$ be an infinite semigroup. Then:

(i) $S^{*}$ is a left ideal in $(\beta S, \square)$ if and only if $S$ is weakly left cancellative; 
(ii) $S^{*}$ is an ideal in $(\beta S, \square)$ if and only if $S$ is weakly cancellative.

Corollary 6.17. Let $S$ be an infinite, weakly cancellative semigroup. Then $\overline{S_{[n]}^{*}}$ is a closed ideal in $(\beta S, \square)$ for each $n \in \mathbb{N}$, and $\overline{S_{[\infty]}^{*}}$ is a closed ideal in $(\beta S, \square)$.

Proof. By Theorem 6.16(ii), $S^{*}$ is an ideal in $\beta S$, and hence so are $S_{[n]}^{*}$ for $n \in \mathbb{N}$, and so is $S_{[\infty]}^{*}$. The result follows from Proposition 6.14(ii).

ExAmple 6.18. Let $G$ be a group, and let $m, n \in \mathbb{N}$. In Chapter 3 , we defined a regular Rees matrix semigroup with zero of the form

$$
S=\mathcal{M}^{o}(G, P, m, n) .
$$

We now consider $\beta S$. First, we see using Proposition 6.5(ii) that the group $G$ acts bijectively on $\beta G$ and $G^{*}$, and so we can consider $\mathcal{M}^{o}\left(\beta G, P_{G}, m, n\right)$ and $\mathcal{M}^{o}\left(G^{*}, P_{G}, m, n\right)$ in our earlier notation for Rees semigroups. In fact, it follows easily from equation (6.1) that, in the notation of (3.2), we have

$$
(\beta S, \square)=\mathcal{M}^{o}\left((\beta G, \square), P_{G}, m, n\right)
$$

and

$$
\left(S^{*}, \square\right)=\mathcal{M}^{o}\left(\left(G^{*}, \square\right), P_{G}, m, n\right),
$$

where $P_{G}=P$. Since $G^{*}$ is an ideal in the semigroup $(\beta G, \square)$, it is clear that $S^{*} \cup\{o\}$ is an ideal in the semigroup $(\beta S, \square)$.

We now define some closed subsets $T_{[n]}^{*}$ in $S^{*}$; they are closely related to the sets $\overline{S_{[n]}^{*}}$.

Definition 6.19. Let $S$ be a semigroup. Set $T_{[1]}^{*}=S^{*}$, and set $T_{[n+1]}^{*}=\overline{S^{*} \square T_{[n]}^{*}}(n \in \mathbb{N})$.

In the case where $S$ is weakly cancellative, each $T_{[n]}^{*}$ is a closed ideal in $\beta S$, and $\overline{S_{[n]}^{*}} \subset T_{[n]}^{*}$. One suspects that in fact $\overline{S_{[n]}^{*}}=T_{[n]}^{*}(n \in \mathbb{N})$. However, this is not necessarily the case: it will be shown in Theorem 6.46, below, that $\overline{\mathbb{N}_{[k+1]}^{*}} \neq \overline{\mathbb{N}^{*} \square \overline{\mathbb{N}}_{[k]}^{*}}$ for $k \geq 2$.

Proposition 6.20. Let $S$ be an infinite, countable, cancellative, unital semigroup.

(i) $\overline{E(K(\beta S))} \backslash S_{[2]}^{*}$ is infinite, so that $K(\beta S)$ and $S_{[2]}^{*}$ are not closed.

(ii) There are right cancellable elements of $\beta S$ in $\overline{E(K(\beta S))}$. 
(iii) There is a dense, open subset $U$ of $S^{*}$ such that each element of $U$ is right cancellable in $(\beta S, \square)$.

Proof. These are Corollaries 8.23 and 8.26 and Theorem 8.10, respectively, of [78].

Proposition 6.21. Let $S$ be an infinite semigroup that is weakly left cancellative and right cancellative. Then $\beta S$ contains right cancellable elements.

Proof. This is [78, Theorem 8.10].

Proposition 6.22. Let $S=(\mathbb{N},+)$ and $T=(\mathbb{N}, \cdot)$. Then the set $\overline{E(K(\beta S))}$ is a left ideal in $(\beta T, \square)$.

Proof. This is [78, Theorem 16.24].

Proposition 6.23. Let $S$ be an infinite semigroup.

(i) Suppose that $S$ is weakly left cancellative, with $|S|=\kappa$. Then $(\beta S, \square)$ contains $2^{2^{\kappa}}$ minimal left ideals, and hence $\beta S$ contains $2^{2^{\kappa}}$ minimal idempotents.

(ii) Suppose that $S$ is cancellative. Then $(\beta S, \square)$ contains at least $2^{\mathfrak{c}}$ minimal right ideals.

Proof. These follow from Theorem 6.42 and Corollaries 6.41 and 6.43 of $[78]$.

Corollary 6.24. Let $S$ be a semigroup such that $(\beta S, \square)$ is a group. Then $S$ is a finite group.

Proof. Since $(\beta S, \square)$ is a group, $S$ is a cancellative semigroup. By clause (ii) of the proposition, $S$ cannot be infinite.

In fact a semigroup $S$ is a finite group whenever $(\beta S, \square)$ is a cancellative semigroup [79].

Proposition 6.25. Let $S$ be an infinite semigroup which is weakly left cancellative and nearly right cancellative. Then $S^{*}$ contains an isomorphic copy of $\mathbb{F}_{2}$ as a subgroup, and $\ell^{1}\left(S^{*}\right)$ contains an isometric and isomorphic copy of $\ell^{1}\left(\mathbb{F}_{2}\right)$ as a closed subalgebra.

Proof. This follows from [78, Lemma 6.31 and Theorem 7.35]; the proof of Lemma 6.31 must be slightly modified. The result was first proved (for the semigroup $\beta \mathbb{N}$ ) in $[\mathbf{7 6}]$; a more general and simpler proof is given in $[\mathbf{1 2 0}]$. 
In the case where the infinite semigroup $S$ is just weakly cancellative, it may be that $S^{*}$ does not contain a copy of $\mathbb{F}_{2}$; for example, this is the case when $S=\mathbb{N}_{\vee}$, as we see from Example 6.3.

Subsets of $S^{*}$ We first characterize the set $S_{[2]}^{*}$.

Proposition 6.26. Let $S$ be an infinite semigroup, and take a subset $A$ of $S$. Then $\bar{A} \cap S_{[2]}^{*} \neq \emptyset$ if and only if there is an infinite subset $T$ of $S$ such that $t_{1}^{-1} A \cap \cdots \cap t_{n}^{-1} A$ is infinite whenever $t_{1}, \ldots, t_{n} \in T$.

Proof. Suppose that there is a set $T$ with the specified property. For each $F \in \mathcal{P}_{f}(T)$, there is $v_{F} \in S^{*}$ with $s v_{F} \in \bar{A}(s \in F)$. Let $v \in S^{*}$ be an accumulation point of the net $\left(v_{F}\right)$. Then $s v \in \bar{A}(s \in T)$, and so $u v \in \bar{A}\left(u \in T^{*}\right)$. In particular, $\bar{A} \cap S_{[2]}^{*} \neq \emptyset$.

Conversely, suppose that there exist $u, v \in S^{*}$ with $u v \in \bar{A}$. Set $T=\{t \in S: t v \in \bar{A}\}$. Then $T \in u$ and $T$ is infinite. For each $t_{1}, \ldots, t_{n} \in T$, we have $t_{1}^{-1} A \cap \cdots \cap t_{n}^{-1} A \in v$, and hence $t_{1}^{-1} A \cap \cdots \cap t_{n}^{-1} A$ is infinite.

The following result was pointed out to us by Yevhen Zelenyuk.

Corollary 6.27. Let $S$ be an infinite, cancellative semigroup. Then $S_{[2]}^{*}$ is not dense in $S^{*}$.

Proof. By Theorem 3.23, $S$ contains an almost left disjoint set, say F. By Proposition 6.26, $\bar{F} \cap S_{[2]}^{*}=\emptyset$.

The next result follows from [78, Theorem 8.22].

Proposition 6.28. Let $S$ an infinite, countable, cancellative semigroup. Then there exists a sequence $\left(p_{n}\right)$ of minimal idempotents in $\beta S$ such that $\overline{\left\{p_{n}: n \in \mathbb{N}\right\}} \cap S_{[2]}^{*}=\left\{p_{n}: n \in \mathbb{N}\right\}$.

Recall from Example 6.3 that we can have $S_{[2]}^{*}=S^{*}$ for a certain weakly cancellative semigroup $S$.

We shall later be concerned with the following subset of $S^{*}$.

Definition 6.29. Let $S$ be a semigroup. Then $D_{S}$ is the subset of $S^{*}$ which is the complement of the union of the sets $F^{*}$ taken over all almost left disjoint

subsets $F$ of $S$.

In the case where $S$ has no almost left disjoint subsets $F$ of $S$, we take $D_{S}=S^{*}$. For example, $D_{S}=S^{*}$ when $S=\mathbb{N}_{\vee}$. Since each set $F^{*}$ is open in $S^{*}$, the set $D_{S}$ is closed in $S^{*}$. 
THEOREM 6.30. Let $S$ be an infinite, weakly cancellative semigroup. Then $\overline{S_{[2]}^{*}} \subset D_{S}$.

Proof. It is sufficient to show that $S_{[2]}^{*} \subset D_{S}$.

Assume towards a contradiction that there exist $u, v \in S^{*}$ and an almost left disjoint subset $F$ of $S$ such that $u \square v \in \bar{F}$. Then we see that $\left\{s \in S: s^{-1} F \in v\right\} \in u$. In particular the set $\left\{s \in S: s^{-1} F \in v\right\}$ contains points $s_{0}$ and $t_{0}$ with $s_{0} \neq t_{0}$. We have $s_{0}^{-1} F \cap t_{0}^{-1} F \in v$, and so $s_{0}^{-1} F \cap t_{0}^{-1} F$ is infinite, a contradiction of the fact that $F$ is an almost left disjoint set.

We are indebted to Imre Leader for the proof of the fact that, at least in the case where $S=\mathbb{N}$, we have $\overline{S_{[2]}^{*}} \subsetneq D_{S}$.

The following result is essentially [78, Theorem 6.35].

Proposition 6.31. Let $S$ be an infinite, weakly cancellative semigroup which is either countable and right cancellative or a subsemigroup of a group. Then the closed set $D_{S}$ has an empty interior with respect to $S^{*}$, and $S_{[2]}^{*}$ is nowhere dense in $S^{*}$.

Proof. Let $V$ be a non-empty, open subset of $S^{*}$. Then there is an infinite subset $X$ of $S$ with $X^{*} \subset V$. By Theorem 3.20 or Theorem 3.22, $X$ contains an almost left disjoint subset, say $F ; F^{*}$ is a non-empty, open subset of $S^{*}$ with $F^{*} \subset V$ and $F^{*} \cap D_{S}=\emptyset$. Thus int $S^{*}\left(D_{S}\right)=\emptyset$. It follows from Theorem 6.30 that $S_{[2]}^{*}$ is nowhere dense in $S^{*}$.

Corollary 6.32. Let $G$ be an infinite group, let $m, n \in \mathbb{N}$, and set $S=\mathcal{M}^{o}(G, P, m, n)$. Then $I=S^{*} \cup\{o\}$ is an ideal in $\beta S$ such that $I_{[2]}$ is nowhere dense in $I$.

Proof. We see that $I_{[2]}=\mathcal{M}\left(\left(G^{*}\right)_{[2]}, P_{G}, m, n\right) \cup\{o\}$, and so this follows from the proposition.

Proposition 6.33. Let $G$ be a countable group or the semigroup $(\mathbb{N},+)$, and let $u, v \in \beta G$ with $(\beta G \square u) \cap(\beta G \square v) \neq \emptyset$. Then either $u \in \beta G \square v$ or $v \in \beta G \square u$.

Proof. This is [78, Corollary 6.20].

Proposition 6.34. Let $G$ be a countable, infinite group. Then there is an almost left disjoint subset $F$ of $G$ such that:

(i) $\left|F^{*}\right|=2^{\mathfrak{c}}$;

(ii) each element of $F^{*}$ is right cancellable in $(\beta G, \square)$; 
(iii) $\left(G \square F^{*}\right) \cap D_{G}=\emptyset$;

(iv) $\left\langle F^{*}\right\rangle$ is a free semigroup in $(\beta G, \square)$.

(v) Suppose further that $G$ is abelian. Then $\beta G \backslash\left(G \square\left\langle F^{*}\right\rangle\right)$ is a prime ideal in $(\beta G, \square)$.

Proof. By Theorem 3.22, there is an almost left disjoint subset $F$ in $G$. By [78, Theorem 6.30] (with $R=G$ and $T=F$ ), we may suppose that each element of $F^{*}$ is right cancellable and that

$$
(\beta G \square u) \cap(\beta G \square v)=\emptyset
$$

whenever $u, v \in F^{*}$ with $u \neq v$. Properties (i) and (ii) are immediate, and (iii) follows because $s F$ is an almost left disjoint set for each $s \in G$.

(iv) Suppose that $e_{1}, \ldots, e_{m}, f_{1}, \ldots, f_{n} \in F^{*}$ and that

$$
e_{1} \square \cdots \square e_{m}=f_{1} \square \cdots \square f_{n} .
$$

We shall show that $m=n$ and that $e_{i}=f_{i}\left(i \in \mathbb{N}_{m}\right)$, which gives the result. Assume towards a contradiction that this is false, and that $m+n$ takes its minimum value for this. We then have $e_{m}=f_{n}$ because otherwise $\left(\beta G \square e_{m}\right) \cap\left(\beta G \square f_{n}\right)=\emptyset$. Since $e_{m}$ is right cancellable, it follows that $e_{1} \square \cdots \square e_{m-1}=f_{1} \square \cdots \square f_{n-1}$. But now $m-1=n-1$ and $e_{i}=f_{i}\left(i \in \mathbb{N}_{m-1}\right)$, as required.

(v) Set $T=\left\langle F^{*}\right\rangle$ and $U=\beta G \backslash(G \square T)$. We claim that $u, v \in G \square T$ whenever $u, v \in \beta G$ and $u \square v \in G \square T$; this is sufficient to show that $U$ is a prime ideal in $\beta G$. It suffices to show that $u, v \in G \square T$ whenever $u, v \in G^{*}$ and $u \square v \in T$.

Choose $u, v \in G^{*}$ and $u_{1}, \ldots, u_{k} \in F^{*}$ such that $u \square v=u_{1} \square \cdots \square u_{k}$ and $k \in \mathbb{N}$ is minimal for such a representation. By (iii) and the fact that $G_{[2]}^{*} \subset D_{G}$, we have $k \geq 2$.

By Proposition 6.33, $v \in \beta G \square u_{k}$ or $u_{k} \in \beta G \square v$. Suppose first that $u_{k}=x \square v$ for some $x \in \beta G$. Since $u_{k} \notin G_{[2]}^{*}$, we have $x \in G$. But now $v \in G \square T$ and, by (ii), $u \in G \square u_{1} \square \cdots \square u_{k-1} \subset G \square T$. Suppose second that $v=x \square u_{k}$ for some $x \in \beta G$. Then we have $u_{1} \square \cdots \square u_{k}=u \square x \square u_{k}$, and so, by (ii) again, $u_{1} \square \cdots \square u_{k-1}=u \square x$. If $x \in G$, then immediately we have $u, v \in G \square T$. If $x \in G^{*}$, then $u, x \in G \square T$ by the minimality of $k$, and then also $v \in G \square T$.

Uniform ultrafilters Let $S$ be an infinite semigroup, and let $\kappa$ be an infinite cardinal with $\kappa \leq|S|$. We shall now obtain some results about the subset $U_{\kappa}(S)$ of $\kappa$-uniform ultrafilters

in $S^{*}$. 
Proposition 6.35. Let $S$ be an infinite semigroup, and let $\kappa$ be an infinite cardinal with $\kappa \leq|S|$. Then the subset $\beta S \backslash U_{\kappa}(S)$ is a subsemigroup of $(\beta S, \square)$ and of $(\beta S, \diamond)$.

Proof. Let $u, v \in \beta S \backslash U_{\kappa}(S)$, and take $U \in u$ and $V \in v$ with $|U|,|V|<\kappa$. Then certainly $U V \in u \square v$ and $|U V|<\kappa$, and so we have $u \square v \in \beta S \backslash U_{\kappa}(S)$. Similarly, $u \diamond v \in \beta S \backslash U_{\kappa}(S)$.

Let $S$ be a weakly cancellative semigroup. The fact that $U_{S}$ is a left ideal in $S^{*}$ is stated in [78, Exercise 6.4.1]. That $U_{S}$ is also a right ideal in $S^{*}$ was first proved in [75]; there is a simpler proof in $[\mathbf{1 5}$, Lemma 3.1], and we essentially repeat this proof below in a slightly more general context. We again write $u v$ for $u \square v$.

Proposition 6.36. Let $S$ be an infinite, weakly cancellative semigroup, and let $\kappa$ be an infinite cardinal with $\kappa \leq|S|$. Then $U_{\kappa}(S)$ is a prime ideal in $(\beta S, \square)$.

Proof. Let $s \in S$ and $x \in U_{\kappa}(S)$. Each member of the ultrafilter $s x$ contains a set of the form $s V$, where $V \in x$. Since $|V| \geq \kappa$, since $V \subset s^{-1}(s V)$, and since $S$ is weakly left cancellative, it follows that $|s V| \geq \kappa$. Thus $s x \in U_{\kappa}(S)$. Since $U_{\kappa}(S)$ is closed in $S^{*}$, it follows that $(\beta S) \square x=\overline{S \square x} \subset U_{\kappa}(S)$, and so $U_{\kappa}(S)$ is a left ideal in $\beta S$.

Now take $x \in U_{\kappa}(S)$ and $y \in \beta S$, and assume towards a contradiction that $x y \notin U_{\kappa}(S)$. Then there is an ultrafilter $W \in x y$ with $|W|<\kappa$. By Proposition 6.4, there exists $U \in x$ and a family $\left\{V_{u}: u \in U\right\}$ with $u V_{u} \subset W(u \in U)$. Since $U_{\kappa}(S)$ is a left ideal, $y \notin U_{\kappa}(S)$, and so there is an ultrafilter $V \in y$ with $|V|<\kappa$.

We claim that $U \subset W V^{-1}$. Indeed, for each $u \in U$, the set $V \cap V_{u}$ belongs to $y$, and so is non-empty. Choose $t \in V \cap V_{u}$. Then $u \in W t^{-1}$, and so the claim holds. Since $S$ is weakly right cancellative, $\left|W t^{-1}\right|<\kappa$ for each $t \in S$, and so $|U| \leq\left|W V^{-1}\right|<\kappa$, a contradiction of the fact that $U \in x$ and $x \in U_{\kappa}(S)$.

Thus $x y \in U_{\kappa}(S)$, and $U_{\kappa}(S)$ is a right ideal, and hence an ideal.

By Proposition $6.35, U_{\kappa}(S)$ is a prime ideal.

We have shown in Proposition 6.31 that, in the case where $S$ is countable, weakly cancellative and right cancellative, $S_{[2]}^{*}$ is a nowhere dense subset of $S^{*}$. The following theorem is more general.

THEOREM 6.37. Let $S$ be an infinite, weakly cancellative semigroup.

(i) Suppose that $S$ is right cancellative. Then $S^{*} U_{S}$ is nowhere dense in $U_{S}$. 
(ii) Suppose that $S$ is left cancellative and that $|S|$ is a regular cardinal. Then $U_{S} S^{*}$ is nowhere dense in $U_{S}$.

Proof. By Proposition 6.36, $U_{S}$ is a ideal in $\beta S$.

We may suppose that $S$ has an identity $e_{S}$. Set $\kappa=|S|$, and enumerate $S$ as $\left\{s_{\alpha}: \alpha<\kappa\right\}$, with $s_{0}=e_{S}$.

Let $V \in[S]^{\kappa}$, so that $\bar{V} \cap U_{S}$ is a non-empty, open subset of $U_{S}$. We shall inductively define a sequence $\left(t_{\sigma}: \sigma<\kappa\right)$ of distinct points in $V$ with $t_{0}=e_{S}$. Suppose that $\tau<\kappa$, and assume that $t_{\sigma}$ has been defined for each $\sigma<\tau$. Set $T_{\tau}=\left\{s_{\sigma}, t_{\sigma}: \sigma<\tau\right\}$ and

$$
W_{\tau}=T_{\tau}^{-1} T_{\tau} T_{\tau}^{-1} T_{\tau}^{3} T_{\tau}^{-1} T_{\tau} T_{\tau}^{-1} .
$$

Since $S$ is weakly cancellative, the set $W_{\tau}$ is finite whenever $\tau<\omega$ and has cardinality $\left|T_{\tau}\right|<\kappa$ whenever $\omega \leq \tau \leq \kappa$. Choose $t_{\tau} \in V \backslash W_{\tau}$. This continues the inductive construction of the sequence $\left(t_{\sigma}: \sigma<\kappa\right)$. Set $T=\left\{t_{\sigma}: \sigma<\kappa\right\}$, so that we have $T^{*} \subset V^{*}$.

(i) Fix $s, t \in S$. We claim that $S^{*} U_{S} \cap s T^{*} t=\emptyset$, which will imply that $s u_{0} t \notin S^{*} U_{S}$ for each $u_{0} \in T^{*}$. In particular, $\overline{S^{*} U_{S}} \cap T^{*}=\emptyset$ and so $\bar{V} \cap U_{S} \not \subset \overline{S^{*} U_{S}}$, and hence (i) holds.

Assume towards a contradiction that there exist $x \in S^{*}$ and $y \in U_{S}$ with $x y \in s T^{*} t$. By Proposition 6.4, there exist $U \in x$ and a family $\left\{V_{u}: u \in U\right\} \subset y$ such that $u V_{u} \subset s T t(u \in U)$.

Certainly the set $U$ contains two distinct points, say $s_{\alpha}, s_{\beta} \in U$ with $\alpha \neq \beta$. We have $s_{\alpha} V_{s_{\alpha}} \cup s_{\beta} V_{s_{\beta}} \subset s T t$. Take $\gamma \geq \max \{\alpha, \beta\}$ such that $s, t \in T_{\gamma}$. Then $\left(s_{\alpha} V_{s_{\alpha}} \cup s_{\beta} V_{s_{\beta}}\right) \backslash s T_{\gamma} t \subset s\left(T \backslash T_{\gamma}\right) t$. However $V_{s_{\alpha}} \cap V_{s_{\beta}}$ belongs to the uniform ultrafilter $y$, and so $\left|V_{s_{\alpha}} \cap V_{s_{\beta}}\right|=\kappa$, whereas $\left|s_{\alpha}^{-1} T_{\alpha}^{3} \cup s_{\beta}^{-1} T_{\beta}^{3}\right|<\kappa$. Thus there exists $r \in V_{s_{\alpha}} \cap V_{s_{\beta}}$ such that $s_{\alpha} r \notin T_{\alpha}^{3}$ and $s_{\beta} r \notin T_{\beta}^{3}$. We have $s_{\alpha} r, s_{\beta} r \in s T t$, and so $s_{\alpha} r=s t_{\sigma} t$ for some $\sigma>\alpha$ and $s_{\beta} r=s t_{\tau} t$ for some $\tau>\beta$. Since $r$ is right cancellable, necessarily $\sigma \neq \tau$, say $\tau>\sigma$. Then $t_{\tau} \in W_{\tau}$, a contradiction of the choice of $T_{\tau}$. The claim is proved.

(ii) Again, fix $s, t \in S$. We claim that $U_{S} S^{*} \cap s T^{*} t=\emptyset$, which will again imply the result.

Assume towards a contradiction that there exist $x \in U_{S}$ and $y \in S^{*}$ with $x y \in s T^{*} t$. By (i), $y \notin U_{S}$, and so there exists $V \in y$ such that $|V|<\kappa$. Since $\kappa$ is a regular cardinal, there exists $\sigma<\kappa$ with $V \cup\{s, t\} \subset S_{\sigma}$, and then $S_{\sigma} \in y$. By Proposition 6.36, xy $\in U_{S}$, and so the set $\left\{s t_{\tau} t: \tau>\sigma\right\}$ belongs to the ultrafilter $x y$. By Proposition 6.4, there exist $U \in x$ and $\left\{V_{u}: u \in U\right\} \subset y$ with

$$
u V_{u} \subset\left\{s t_{\tau} t: \tau>\sigma\right\} \quad(u \in U) .
$$


Take $u \in U$. Since $V_{u} \cap Y \in y$, we may choose $s_{\alpha}, s_{\beta} \in V_{u} \cap V$ with $\alpha \neq \beta$. Since $V \subset S_{\sigma}$, we have $\alpha, \beta \leq \sigma$. Further, $u s_{\alpha}=s t_{\tau} t$ and $u s_{\beta}=s t_{\rho} t$ for some $\tau, \rho \in(\sigma, \kappa)$. Since $u$ is left cancellable, necessarily $\tau \neq \rho$, say $\tau<\rho$. We have $\alpha, \beta \leq \tau$, and so $u \in T_{\rho}^{-1} T_{\rho}^{3}$ and $t_{\rho} \in T_{\rho}^{-1} T_{\rho}^{-1} T_{\rho}^{3} T_{\rho} T_{\rho}^{-1} \subset W_{\rho}$, a contradiction of the choice of $t_{\rho}$. The claim is proved.

Special subsets of $\beta \mathbb{N}$ There are certain subsets of $\beta \mathbb{N}=(\beta \mathbb{N}, \square)$ to which we shall refer; for a full discussion of these sets, see [78].

Let $G$ be a group, and take $u \in \beta G$. Then $\left\{U^{-1}: U \in u\right\}$ is an ultrafilter in $\beta G$. In the case where $(G,+)$ is abelian, we denote this element of $\beta G$ by $(-1) \cdot u$.

Proposition 6.38. (i) Each element of $\mathbb{N}^{*} \backslash \mathbb{N}_{[2]}^{*}$ is right cancellable.

(ii) There exists an element a of $\mathbb{N}_{[\infty]}^{*}$ such that a is right cancellable.

Proof. (i) This follows from [78, Theorem 8.18].

(ii) Take $p \in E\left(\mathbb{N}^{*}\right)$ with $p \in \overline{F S\left\langle\left(3^{n}: n \geq m\right)\right\rangle}(m \in \mathbb{N})$, and set $a=((-1) \cdot p) \square p$ in $\beta \mathbb{Z}$. Then $a \in \mathbb{N}_{[\infty]}^{*}$. The proof that $a$ is right cancellable in $\beta \mathbb{N}$ is contained within that of [78, Example 8.29].

DeFinition 6.39. Let

$$
\mathbb{P}=\left\{2^{n}: n \in \mathbb{N}\right\} \quad \text { and } \quad \mathbb{H}=\bigcap\left\{\overline{2^{n} \mathbb{N}}: n \in \mathbb{N}\right\}
$$

Proposition 6.40. We have:

(i) $\left|\mathbb{P}^{*}\right|=2^{\mathfrak{c}}$;

(ii) $\left\langle\mathbb{P}^{*}\right\rangle$ is a free subsemigroup of $\beta \mathbb{N}$;

(iii) each element of $\mathbb{P}^{*}$ is cancellable in $\beta \mathbb{N}$;

(iv) $\left(\mathbb{Z} \square \mathbb{P}^{*}\right) \cap D_{\mathbb{N}}=\emptyset$;

(v) $\beta \mathbb{Z} \backslash\left(\mathbb{Z} \square\left\langle\mathbb{P}^{*}\right\rangle\right)$ is a prime ideal in $(\beta \mathbb{Z}, \square)$.

Proof. This is essentially the same as the proof of Proposition 6.34: the fact that we can choose $G=\mathbb{Z}$ and $F=\mathbb{P}$ in that proposition follows from [78, Corollary 8.38], which shows that each element of $\mathbb{P}^{*}$ is cancellable in $(\beta \mathbb{Z}, \square)$, and [78, Lemma 6.8], which implies that

$$
(\beta \mathbb{N} \square u) \cap(\beta \mathbb{N} \square v)=\emptyset
$$

whenever $u, v \in \mathbb{P}^{*}$ with $u \neq v$. 
Proposition 6.41. The set $\mathbb{H}$ is a compact subsemigroup of $\mathbb{N}^{*}$ containing all the idempotents of $\beta \mathbb{N}$; there are $2^{\mathfrak{c}}$ idempotents in $\mathbb{H}$; $\mathbb{H}$ contains an infinite decreasing sequence of idempotents; if $u, v \in \mathbb{N}^{*}$ and $u \square v, v \in \mathbb{H}$, then $u \in \mathbb{H}$.

Proof. This is contained in [78, Chapter 6.1].

We shall now prove that $\overline{\mathbb{N}_{[3]}^{*}} \subsetneq \overline{\mathbb{N}^{*} \square \overline{\mathbb{N}_{[2]}^{*}}}$, and indeed the more general fact that $\overline{\mathbb{N}_{[k+1]}^{*}} \subsetneq \overline{\mathbb{N}^{*} \square \overline{\mathbb{N}_{[k]}^{*}}}$ for each $k \geq 2$. We are grateful to Neil Hindman for an essential contribution to the proof of the following theorem. We first introduce some notation.

Each number $n \in \mathbb{N}$ has a unique expression in the form

$$
n=\sum_{i=0}^{\infty} \varepsilon_{i}(n) 2^{i},
$$

where $\varepsilon_{i}(n) \in\{0,1\}$ and $\varepsilon_{i}(n)=0$ eventually. We set

$$
\operatorname{supp} n=\left\{i \in \mathbb{N}: \varepsilon_{i}(n)=1\right\} \text {, }
$$

and define $\gamma: n \mapsto|\operatorname{supp} n|, \quad \mathbb{N} \rightarrow \mathbb{N}$. The map $\gamma$ has a continuous extension to a map $\gamma: \beta \mathbb{N} \rightarrow \beta \mathbb{N}$. Clearly $\overline{\mathbb{P}}=\{u \in \beta \mathbb{N}: \gamma(u)=1\}$. We also define

$$
\varepsilon: n \mapsto\left(\varepsilon_{i}(n): i \in \mathbb{N}\right), \quad \mathbb{N} \rightarrow\{0,1\}^{\omega},
$$

and extend $\varepsilon$ to a continuous map $\varepsilon: \beta \mathbb{N} \rightarrow\{0,1\}^{\omega}$. Observe that the map $\varepsilon$ is a homomorphism from the semigroup $(\beta \mathbb{N}, \square)$ onto the group of 2-adic integers. We shall use the following easily checked facts. First, we have

$$
\gamma(u \square v)=\gamma(u) \square \gamma(v) \quad(u \in \beta \mathbb{N}, v \in \mathbb{H}) .
$$

Second, let $u \in \beta \mathbb{N}$. For each $n \in \mathbb{N}$, we have $u \in \overline{2^{n} \mathbb{N}}$ if and only if $\varepsilon_{0}(u)=\cdots=\varepsilon_{n-1}(u)=0$. It follows that $u \in \mathbb{H}$ if and only if $\varepsilon(u)=0$. It is now easy to see that, if $u \in \mathbb{N}^{*}$ and $\varepsilon(u)$ contains only finitely many 0 's or only finitely many 1 's, then $u \in \mathbb{Z} \square \mathbb{H}$.

We shall also require three lemmas; we maintain the above notation.

Lemma 6.42. Let $u, v \in \mathbb{N}^{*}$ with $\gamma(u \square v) \in \mathbb{N}$. Then $n \square v \in \mathbb{H}$ for some $n \in \mathbb{Z}$.

Proof. Set $m=\gamma(u \square v)$ and $B=\{n \in \mathbb{N}: \gamma(n)=m\}$, so that $u \square v \in \bar{B}$. Since $R_{v}$ is continuous and $\bar{B}$ is open in $\beta \mathbb{N}$, there exists $k \in \mathbb{N}$ such that $k \square v \in \bar{B}$, so that $\gamma(k \square v)=m$. 
Assume towards a contradiction that $v \notin \mathbb{Z} \square \mathbb{H}$. Then the sequence $\varepsilon(v)$ contains infinitely many 0 's; choose

$$
i>\max \{\operatorname{supp} k\} \quad \text { with } \quad \varepsilon_{i}(v)=0 .
$$

Clearly $\varepsilon_{j}(k \square v)=\varepsilon_{j}(v) \quad(j>i)$. Since $\varepsilon_{r}(k \square v)$ is equal to 1 for exactly $m$ values of $r$, the sequence $\varepsilon(v)$ contains only finitely many 1 's, and so $v \in \mathbb{Z} \square \mathbb{H}$.

The result follows.

Lemma 6.43. Let $u_{1}, \ldots, u_{k} \in \mathbb{N}^{*}$, and set $u=u_{1} \square \cdots \square u_{k}$.

(i) Suppose that $\gamma(u) \in \mathbb{N}$. Then $\gamma(u) \geq k$.

(ii) Suppose that $\gamma(u)=k$. Then $u \in \mathbb{H}_{[k]}$.

Proof. (i) The proof is by induction on $k$. The result is clear for $k=1$. Suppose that $k \geq 2$, and assume that the result holds for $k-1$. By Lemma 6.42, there exists $n \in \mathbb{Z}$ such that $n \square u_{k} \in \mathbb{H}$. By replacing $u_{k}$ by $n \square u_{k}$ and $u_{k-1}$ by $(-n) \square u_{k-1}$, we may suppose that $u_{k} \in \mathbb{H}$. Now, by (6.3),

$$
\gamma(u)=\gamma\left(u_{1} \square \cdots \square u_{k-1}\right) \square \gamma\left(u_{k}\right) .
$$

Since $\gamma(u) \in \mathbb{N}$, we have $\gamma\left(u_{1} \square \cdots \square u_{k-1}\right) \in \mathbb{N}$ and $\gamma\left(u_{k}\right) \in \mathbb{N}$. By the inductive hypothesis, $\gamma\left(u_{1} \square \cdots \square u_{k-1}\right) \geq k-1$, and $\gamma\left(u_{k}\right) \geq 1$, and so $\gamma(u) \geq k$, continuing the induction.

(ii) Again the result is clear for $k=1$ because $\mathbb{P}^{*} \subset \mathbb{H}$. Suppose that $k \geq 2$, and assume that the result holds for $k-1$. Again, we may suppose that $u_{k} \in \mathbb{H}$ and that (6.4) holds. Since $\gamma(u) \in \mathbb{N}$, necessarily $\gamma\left(u_{1} \square \cdots \square u_{k-1}\right), \gamma\left(u_{k}\right) \in \mathbb{N}$. By $(\mathrm{i})$,

$$
\gamma\left(u_{1} \square \cdots \square u_{k-1}\right) \geq k-1 .
$$

Thus $\gamma\left(u_{k}\right)=1$ and $\gamma\left(u_{1} \square \cdots \square u_{k-1}\right)=k-1$. By the inductive hypothesis, $u_{1} \square \cdots \square u_{k-1} \in \mathbb{H}_{[k-1]}$, and so $u \in \mathbb{H}_{[k]}$.

The result follows by induction on $k$.

Corollary 6.44. Let $k \in \mathbb{N}$. Then $\overline{\mathbb{N}_{[k+1]}^{*}} \subsetneq \overline{\mathbb{N}_{[k]}^{*}}$.

Proof. Certainly $\overline{\mathbb{N}_{[k+1]}^{*}} \subset \overline{\mathbb{N}_{[k]}^{*}}$. It follows from Lemma 6.43(i) that $\gamma(u) \geq k+1$ for each $u \in \overline{\mathbb{N}_{[k+1]}^{*}}$. However, let $u \in \mathbb{P}_{[k]}^{*} \subset \overline{\mathbb{N}_{[k]}^{*}}$. Then $\gamma(u)=k$. The result follows. 
We now fix $k \in \mathbb{N}$ with $k \geq 2$. Let $\left\{E_{n}: n \in \mathbb{N}\right\}$ be a partition of $\mathbb{N}$ into infinite subsets. For $j, r \in \mathbb{N}$, set

$$
\begin{aligned}
A_{j, r}=\left\{2^{j}+2^{m_{1}}+2^{m_{2}}+\cdots+2^{m_{k-1}}+2^{m_{k}}:\right. & \\
m_{k}>m_{k-1} & \left.>\cdots>m_{2}>m_{1}>j, \quad m_{k-1}, m_{k} \in E_{r}\right\}
\end{aligned}
$$

and then set

$$
A=\bigcup\left\{A_{j, r}: j, r \in \mathbb{N}, j<r\right\} .
$$

We see that $\gamma(u)=k+1(u \in \bar{A})$

Lemma 6.45. (i) $\bar{A} \cap \mathbb{H}_{[k+1]}=\emptyset$.

(ii) $\bar{A} \cap\left(\mathbb{H} \square \overline{\mathbb{H}_{[k]}}\right) \neq \emptyset$.

Proof. (i) Assume towards a contradiction that $u_{1}, \ldots, u_{k+1} \in \mathbb{H}$ with $u \in \bar{A}$, where $u=u_{1} \square \cdots \square u_{k+1}$. It follows from equation (6.3) that $u_{1}, \ldots, u_{k+1} \in \mathbb{P}^{*}$. Since $u \in \bar{A}$ and $R_{v}$ is continuous for each $v \in \beta S$, we can successively choose $m_{0}, m_{1}, \ldots, m_{k-1} \in \mathbb{N}$ such that $m_{k-1}>\cdots>m_{1}>m_{0}$ and $\left(2^{m_{0}}+2^{m_{1}}+\cdots+2^{m_{k-1}}\right) \square u_{k+1} \in \bar{A}$, and so

$$
\left\{2^{s}: s>m_{k-1}, 2^{m_{0}}+2^{m_{1}}+\cdots+2^{m_{k-1}}+2^{s} \in A\right\} \in u_{k+1} .
$$

Now choose $r \in \mathbb{N}$ such that $m_{k-1} \in E_{r}$. It follows from the definition of $A$ that $u_{k+1} \in E_{r}^{*}$. Note that this shows that $r$ is independent of the choice of $m_{0}, m_{1}, \ldots, m_{k-1}$. We see that

$$
A \cap\left\{2^{n_{0}}+2^{n_{1}}+\cdots+2^{n_{k}}: n_{k}>\cdots>n_{1}>n_{0}, n_{k} \in E_{r}\right\} \in u .
$$

Again from the fact that $j<r$ in the definition of $A$, we have

$$
\left\{2^{n_{0}}+2^{n_{1}}+\cdots+2^{n_{k}}: n_{k}>\cdots>n_{1}>n_{0}, n_{0}<r, n_{k} \in E_{r}\right\} \in u \text {. }
$$

Thus there exists $j \in \mathbb{N}$ with $j<r$ such that

$$
B:=\left\{2^{j}+2^{n_{1}}+\cdots+2^{n_{k}}: n_{k}>\cdots>n_{1}>j\right\} \in u .
$$

However this is a contradiction because $2^{r} \mathbb{N} \in u$ and $B \cap 2^{r} \mathbb{N}=\emptyset$.

(ii) In this proof, we set $\mathbb{P}_{[0]}=\{0\}$. For $j \in \mathbb{N}$, define

$$
X_{j}=\overline{\bigcup\left\{\mathbb{P}_{[k-2]}^{*} \square E_{r}^{*} \square E_{r}^{*}: r>j\right\}},
$$

so that $\left(X_{j}\right)$ is a decreasing sequence of non-empty, closed subsets of $\mathbb{H}_{[k]}^{*}$. Choose $v \in \bigcap\left\{X_{j}: j \in \mathbb{N}\right\}$.

Let $j \in \mathbb{N}$. We claim that $2^{j} \square v \in \bar{A}$. Indeed, choose $B \in v$. Since $v \in X_{j}$, there exist $r>j, x \in \mathbb{P}_{[k-2]}^{*}$, and $y, z \in E_{r}^{*}$ with $x \square y \square z \in \bar{B}$. Now $2^{j} \square x \square y \square z \in \overline{A_{j, r}} \subset \bar{A}$, and so $\left(2^{j} \square \bar{B}\right) \cap \bar{A} \neq \emptyset$, as claimed.

Choose $w \in \mathbb{P}^{*}$. Then $w \square v \in \mathbb{H} \square \overline{\mathbb{H}_{[k]}}$. Since $2^{j} \square v \in \bar{A}(j \in \mathbb{N})$ and $R_{v}$ is continuous, we also have $w \square v \in \bar{A}$. Thus (ii) follows. 
Theorem 6.46. Let $k \in \mathbb{N}$ with $k \geq 2$. Then

$$
\overline{\mathbb{N}_{[k+1]}^{*}} \subsetneq \overline{\mathbb{N}^{*} \square \overline{\mathbb{N}_{[k]}^{*}}} \text {. }
$$

ProOF. It is immediate that $\overline{\mathbb{N}_{[k+1]}^{*}} \subset \overline{\mathbb{N}^{*} \square \overline{\mathbb{N}_{[k]}^{*}}}$.

Let $A$ be the set defined above. By Lemma 6.45(i), $\bar{A} \cap \mathbb{H}_{[k+1]}=\emptyset$. It now follows from Lemma 6.43(i), that $\bar{A} \cap \mathbb{N}_{[k+1]}^{*}=\emptyset$. On the other hand, by Lemma 6.45(ii), $\bar{A} \cap\left(\mathbb{H} \square \overline{\mathbb{H}}_{[k]}\right) \neq \emptyset$, and so we have $\bar{A} \cap\left(\overline{\mathbb{N}^{*} \square \overline{\mathbb{N}_{[k]}^{*}}}\right) \neq \emptyset$. Thus $\overline{\mathbb{N}_{[k+1]}^{*}} \neq \overline{\mathbb{N}^{*} \square \overline{\mathbb{N}_{[k]}^{*}}}$. 



\section{CHAPTER 7}

\section{Second duals of semigroup algebras}

Let $S$ be a semigroup, and consider the Banach algebra $A=\left(\ell^{1}(S), \star\right)$. Throughout this chapter, we shall consider the second duals $\left(A^{\prime \prime}, \square\right)$ and $\left(A^{\prime \prime}, \diamond\right)$ of $A$; the definitions of these second duals were given in Chapter 2. We shall also consider the closed subalgebras $\left(\ell^{1}(\beta S), \square\right)$ and $\left(\ell^{1}(\beta S), \diamond\right)$ of these algebras.

The Banach algebra $M(\beta S)$ We first introduce a Banach algebra that will be at the centre of much of the remainder of this memoir.

Definition 7.1. Let $S$ be a semigroup. Then $M(\beta S)$ denotes the Banach space of complex regular Borel measures

on $\beta S$, and the two products $\square$ and $\diamond$ on $M(\beta S)$ are defined by identifying $M(\beta S)$ with $\ell^{1}(S)^{\prime \prime}$.

Thus we have definitions of

$$
\mu \square \nu \text { and } \mu \diamond \nu \text { for } \mu, \nu \in M(\beta S) .
$$

We shall often write $u \square \mu$ for $\delta_{u} \square \mu$, etc., in the case where $u \in \beta S$ and $\mu \in M(\beta S)$.

The first paper to consider the Banach algebra $\left(\ell^{1}(S)^{\prime \prime}, \square\right)$ is probably [29]. For an early survey which includes a discussion of the Banach algebras $(M(\beta S), \square)$ and $(M(\beta S), \diamond)$, see $[31]$. The above formulation of the two products on $M(\beta S)$ is given in [115] (in a special case).

The element of $\ell^{\infty}(S)$ or $C(\beta S)$ which is constantly equal to 1 is denoted by 1 . Note that the augmentation character

on $\ell^{1}(S)$ corresponds to the constant function 1 in $\ell^{\infty}(S)$, and the second dual of this character on $M(\beta S)$ corresponds to the constant function 1 in $C(\beta S)$; indeed this latter character is the map

$$
\varphi_{S}^{\prime \prime}: \mu \mapsto\langle\mu, 1\rangle=\mu(\beta S), \quad M(\beta S) \rightarrow \mathbb{C} .
$$

We shall require the following technical result about elements of $M(\beta S)$. The family of Borel subsets

of $\beta S$ is denoted by $\mathcal{B}_{\beta S}$.

Proposition 7.2. Let $S$ be a left cancellative semigroup.

(i) For $B \in \mathcal{B}_{\beta S}$ and $s \in S$, the set $s B \in \mathcal{B}_{\beta S}$. 
(ii) For $\mu \in M(\beta S)$ and $s \in S$, set $\mu_{s}(B)=\mu(s B)\left(B \in \mathcal{B}_{\beta S}\right)$. Then $\mu_{s} \in M(\beta S)$. Further, $(\mu \square \nu)_{s}=\mu_{s} \square \nu(\mu, \nu \in M(\beta S))$.

Proof. (i) The family $\left\{E \subset \beta S: s E \in \mathcal{B}_{\beta S}\right\}$ contains the compact subsets of $\beta S$ and is closed under countable unions and complementation because $S$ is left cancellative, and so the family contains $\mathcal{B}_{\beta S}$.

(ii) Clearly $\mu_{s}$ is a Borel measure.

To see that $\mu_{s}$ is regular, take $B \in \mathcal{B}_{\beta S}$ and $\varepsilon>0$. Choose a compact subset $K$ of $s B$ with $|\mu(s B)-\mu(K)|<\varepsilon$, and set $L=s^{-1} K$. Then $L$ is compact and $s L=K$, and so $\left|\mu_{s}(B)-\mu_{s}(L)\right|<\varepsilon$. Thus $\mu_{s} \in M(\beta S)$.

For each $s \in S$, the map $\mu \mapsto \mu_{s}$ is weak-* continuous on $M(\beta S)$ because $s B$ is clopen in $\beta S$ whenever $B$ is clopen in $\beta S$. Let $f \in \ell^{1}(S)$, and take $s, t \in S$ and $B \in \mathcal{B}_{\beta S}$. Then

$$
\left(f_{s} \square t\right)(B)=f\left(s B t^{-1}\right)=(f \square t)(s B)=(f \square t)_{s}(B),
$$

and so $f_{s} \square t=(f \square t)_{s}$. It follows successively that $f_{s} \square \nu=(f \square \nu)_{s}$ for $\nu \in M(\beta S)$, and then that $(\mu \square \nu)_{s}=\mu_{s} \square \nu$ for $\mu, \nu \in M(\beta S)$.

Introverted $C^{*}$-subalgebras We have defined introverted submodules in Definition 2.30. We now give a variant of this definition.

Definition 7.3. Let $S$ be a semigroup. A closed subspace $X$ of $\ell^{\infty}(S)$ is a left-introverted $C^{*}$-subalgebra

if:

(i) $X$ is a $C^{*}$-subalgebra of $\ell^{\infty}(S)$;

(ii) $X$ is a left-introverted submodule of $\ell^{\infty}(S)$, where $\ell^{\infty}(S)$ is regarded as the dual module of $\ell^{1}(S)$.

Similarly, we define a right-introverted $C^{*}$-subalgebra of $\ell^{\infty}(S)$. The space $X$ is an introverted $C^{*}$-subalgebra if it is both a left- and a rightintroverted $C^{*}$-subalgebra.

In particular, $\ell^{\infty}(S)$ itself is an introverted $C^{*}$-subalgebra of $\ell^{\infty}(S)$. Further examples will be given in Chapter 8 .

Let $X$ be a $C^{*}$-subalgebra of $\ell^{\infty}(S)$. The character space of $X$ is denoted by $\Phi_{X}$. (In the case where $X=\ell^{\infty}(S)$, of course $\Phi_{X}=\beta S$.)

Let $X$ be a left-introverted $C^{*}$-subalgebra of $\ell^{\infty}(S)$. As in Chapter 2 , the space $X^{\prime}$ is a Banach algebra for the product $\square$ specified in equation (2.16). This product gives a product, also denoted by $\square$, on $\Phi_{X}$, and we claim that the compact space $\Phi_{X}$ is a semigroup with respect to $\square$. Indeed, let $u, v \in \Phi_{X}$, regarding $u$ and $v$ as characters on $X$. Take $s \in S$, and let $\lambda \in X$. Then $\left\langle\delta_{s}, \delta_{v} \cdot \lambda\right\rangle=\left\langle\delta_{v}, \lambda \cdot \delta_{s}\right\rangle$ 
and $\left\langle\delta_{u} \square \delta_{v}, \lambda\right\rangle=\left\langle\delta_{u}, \delta_{v} \cdot \lambda\right\rangle$ by equation (2.16). Now take $\lambda_{1}, \lambda_{2} \in X$. Then it follows that

$$
\lambda_{1} \lambda_{2} \cdot \delta_{s}=\left(\lambda_{1} \cdot \delta_{s}\right)\left(\lambda_{2} \cdot \delta_{s}\right),
$$

and so $\delta_{v} \cdot \lambda_{1} \lambda_{2}=\left(\delta_{v} \cdot \lambda_{1}\right)\left(\delta_{v} \cdot \lambda_{2}\right)$. Hence we have

$$
\left\langle\delta_{u} \square \delta_{v}, \lambda_{1} \lambda_{2}\right\rangle=\left\langle\delta_{u} \square \delta_{v}, \lambda_{1}\right\rangle\left\langle\delta_{u} \square \delta_{v}, \lambda_{2}\right\rangle,
$$

and so $\delta_{u} \square \delta_{v}$ is a character on $X$, necessarily of the form $\delta_{x}$ for some $x \in \Phi_{X}$. Thus $\left(\Phi_{X}, \square\right)$ is a semigroup, and hence it is a compact, right topological semigroup.

There is semigroup morphism $\gamma_{X}: S \rightarrow \Phi_{X}$. Suppose that $E:=$ $c_{0}(S) \subset X$, so that $X$ separates the points of $S$. Then the map $\gamma_{X}$ is an injection, and we can regard $S$ as a subset of $\Phi_{X}$. Clearly $S$ is dense in $\Phi_{X}$, and so $\Phi_{X}$ is a compactification of $S$. As in equation (2.17), we have

$$
X^{\prime}=\ell^{1}(S) \ltimes\left(E^{\circ} / X^{\circ}\right) .
$$

We have already defined a quotient map $\pi_{X}: A^{\prime \prime} \rightarrow X^{\prime}$. This induces a continuous semigroup homomorphism

$$
\pi_{X}:(\beta S, \square) \rightarrow\left(\Phi_{X}, \square\right) .
$$

Let $\left(s_{\alpha}\right)$ and $\left(t_{\beta}\right)$ be nets in $S$ such that $\lim _{\alpha} s_{\alpha}=u$ and $\lim _{\beta} t_{\beta}=v$ in $\Phi_{X}$. Then $\lim _{\alpha} \delta_{s_{\alpha}}=\delta_{u}$ and $\lim _{\beta} \delta_{t_{\beta}}=\delta_{v}$ in the weak-* topology of $X^{\prime \prime}$, and so

$$
\delta_{u} \square \delta_{v}=\lim _{\alpha} \lim _{\beta} \delta_{s_{\alpha}} \star \delta_{t_{\beta}}=\lim _{\alpha} \lim _{\beta} \delta_{s_{\alpha} t_{\beta}}=\delta_{u \square v} .
$$

Thus it is consistent to identify $\delta_{u} \square \delta_{v}$ with the point $x=u \square v$ of $\Phi_{X}$ : the compact, right topological semigroup $\left(\Phi_{X}, \square\right)$ is identified with a subsemigroup of $\left(X^{\prime}, \square\right)$.

Let $X$ be a right-introverted $C^{*}$-subalgebra of $\ell^{\infty}(S)$. Then, similarly, $\left(\Phi_{X}, \diamond\right)$ is a compact, left topological subsemigroup of $\left(X^{\prime}, \diamond\right)$, and

$$
u \diamond v=\lim _{\beta} \lim _{\alpha} s_{\alpha} t_{\beta} \quad \text { in } \Phi_{X} .
$$

In particular, $(\beta S, \diamond)$ is a compact, left topological semigroup.

The above was first proved by Civin and Yood [16] in the case where $X=\ell^{\infty}(S)$. In particular, our two definitions of the product in $\beta S$ are consistent. Clearly

$$
\left(\mathfrak{Z}_{t}^{(\ell)}(M(\beta S)) \cap \beta S\right) \subset \mathfrak{Z}_{t}^{(\ell)}(\beta S) ;
$$

we do not know an example where this inclusion is proper.

For examples and further discusssion of introverted $C^{*}$-subalgebras of $\ell^{\infty}(S)$, see Chapter 8 . 
Spectra The first result concerns the spectra

of elements in the algebras $\ell^{1}(\beta S)$ and $M(\beta S)$.

Proposition 7.4. Let $S$ be a non-unital semigroup, and let $s \in S^{*}$. Set $A=\ell^{1}(S), L=\ell^{1}(\beta S)$, and $M=M(\beta S)=A^{\prime \prime}$. Then:

(i) in the finite case, $\sigma_{L}(s)=\sigma_{M}(s)=\left\{0,1, \zeta, \ldots, \zeta^{k-1}\right\}$, where $k$ is the period of $s$ and $\zeta=\exp (2 \mathrm{i} \pi / k)$;

(ii) in the infinite case, $\sigma_{L}(s)=\sigma_{M}(s)=\overline{\mathbb{D}}$.

Proof. The results for $\sigma_{L}(s)$ are immediate from Proposition 4.5. The results for $\sigma_{M}(s)$ then follow from Proposition 2.21(i).

Closed subalgebras and closed ideals Now we consider closed subalgebras and closed ideals in the specified Banach algebras.

Let $S$ be a semigroup, and let $\mu \in M(\beta S), s \in S$, and $v \in \beta S$. Then

$$
\operatorname{supp}(s \cdot \mu)=s \cdot \operatorname{supp} \mu, \quad \operatorname{supp}(\mu \square v) \subset(\operatorname{supp} \mu) \square v ;
$$

in the case where either $\mu \in M(\beta S)^{+}$or $v$ is right cancellable in $\beta S$, it follows from Proposition 4.4 that

$$
\operatorname{supp}(\mu \square v)=(\operatorname{supp} \mu) \square v .
$$

It is very tempting to think that we should also have the inclusion $\operatorname{supp}(v \square \mu) \subset v \square(\operatorname{supp} \mu)$, or at least $\operatorname{supp}(v \square \mu) \subset \overline{v \square(\operatorname{supp} \mu)}$. However, this latter inclusion may fail, even in the case where $S=\mathbb{N}$, as we shall see in Proposition 9.27, below.

Let $T$ be a subsemigroup of a semigroup $S$. The isometric embedding of $\ell^{1}(T)$ into $\ell^{1}(S)$ extends to an isometric embedding of the Banach algebras $(M(\beta T), \square)$ and $(M(\beta T), \diamond)$ into $(M(\beta S), \square)$ and $(M(\beta S, \diamond)$, respectively; the image of $\mu \in M(\beta T)$ in $M(\beta S)$ is just the measure $\widetilde{\mu}$ defined by setting $\widetilde{\mu}(B)=\mu(B \cap \bar{T})$ for a Borel subset $B$ of $\beta S$, and we shall identify $\mu$ and $\widetilde{\mu}$.

Let $S$ and $T$ be semigroups, and let $\theta: S \rightarrow T$ be an epimorphism. Then there is an induced continuous epimorphism $\theta: \ell^{1}(S) \rightarrow \ell^{1}(T)$, and hence a continuous epimorphism $\theta^{\prime \prime}:(M(\beta S), \square) \rightarrow(M(\beta T), \square)$. Suppose that there is a non-zero, continuous point derivation on the algebra $(M(\beta T), \square)$. Then, by Proposition 2.2(ix), there is a non-zero, continuous point derivation on $(M(\beta S), \square)$.

Proposition 7.5. Let $S$ be a semigroup.

(i) Let $L$ be a closed, left ideal in $(\beta S, \square)$. Then $M(L)$ is a weak*-closed left ideal in $(M(\beta S), \square)$. 
(ii) Let $U$ and $V$ be subsets of $S$, and let $\mu \in M(\bar{U})$ and $\nu \in M(\bar{V})$. Then $\mu \square \nu \in M(\bar{U} \square \bar{V})$.

(iii) Let I be a left (respectively, right) ideal in $S$. Then $M(\beta I)$ is a left (respectively, right) ideal in $(M(\beta S), \square)$.

(iv) Let $I$ be an ideal in $S$. Then there is a continuous epimorphism from $\left(\ell^{1}(\beta S), \square\right)$ onto $\left(\ell^{1}(\beta(S / I)), \square\right)$ and from $(M(\beta S), \square)$ onto $(M(\beta(S / I)), \square)$.

Proof. (i) We have noted that $M(L)$ is a weak-*-closed subspace of the Banach space $M(\beta S)$.

Let $\mu \in M(\beta S)$ and $\nu \in M(L)$. Then there are nets $\left(f_{\alpha}\right)$ in $\ell^{1}(S)$ and $\left(g_{\beta}\right)$ in $\ell^{1}(L)$ such that $\mu=\lim _{\alpha} f_{\alpha}$ and $\nu=\lim _{\beta} g_{\beta}$. Since $L$ is a left ideal in $\beta S$, we have $f_{\alpha} \square g_{\beta} \in \ell^{1}(L) \subset M(L)$. Also we have $f_{\alpha} \square \nu=\lim _{\beta} f_{\alpha} \square g_{\beta} \in M(L)$ for each $\alpha$, and hence

$$
\mu \square \nu=\lim _{\alpha} f_{\alpha} \square \nu \in M(L)
$$

because $R_{\nu}$ is continuous. Thus $M(L)$ is a left ideal in $(M(\beta S), \square)$.

(ii) This is similar.

(iii) This follows from (ii).

(iv) There is an epimorphism $\theta: S \rightarrow S / I$, and so there is a continuous epimorphism $\theta: \ell^{1}(S) \rightarrow \ell^{1}(S / I)$. We can identify $\beta(S / I)$ with $\beta S / \beta I$, and so it follows that we have a continuous epimorphism $\theta^{\prime \prime}:(M(\beta S), \square) \rightarrow(M(\beta(S / I)), \square)$. Clearly the map

$$
\theta^{\prime \prime} \mid \ell^{1}(\beta S): \ell^{1}(\beta S) \rightarrow \ell^{1}(\beta(S / I))
$$

is a continuous epimorphism.

For example, $M\left(\mathbb{Z}^{+*}\right)$ and $M\left(\mathbb{Z}^{-*}\right)$ are non-zero, closed left ideals in $(M(\beta \mathbb{Z}), \square)$ with $M\left(\mathbb{Z}^{*}\right)=M\left(\mathbb{Z}^{+*}\right) \oplus M\left(\mathbb{Z}^{-*}\right)$. However we shall show in Proposition 9.28 that $M(R)$ is not necessarily a right ideal in $(M(\beta S), \square)$ whenever $R$ is a closed right ideal in $\beta S$.

Proposition 7.6. Let $S$ be an infinite, weakly left cancellative semigroup. Then $M\left(\overline{S_{[n]}^{*}}\right)$ is a $\|\cdot\|$-closed left ideal in $M(\beta S, \square)$ for each $n \in \mathbb{N}$, and $M\left(\overline{S_{[\infty]}^{*}}\right)$ is a $\|\cdot\|$-closed left ideal in $M(\beta S, \square)$.

Proof. By Theorem 6.16(i), $S^{*}$ is a left ideal in $\beta S$, and so each of $S_{[n]}^{*}$ and $S_{[\infty]}^{*}$ is a left ideal in $\beta S$. By Proposition 6.14(i), each of $\overline{S_{[n]}^{*}}$ and $\overline{S_{[\infty]}^{*}}$ is a left ideal in $\beta S$. The result now follows from Proposition $7.5(\mathrm{i})$.

Proposition 7.7. Let $S$ be a semigroup. Then $\overline{M\left(S^{*}\right)^{2}} \subset M\left(\overline{S_{[2]}^{*}}\right)$. 
Proof. We may suppose that $S$ is infinite, for otherwise the result is trivial.

Take $U \subset S$ with $\bar{U} \cap S_{[2]}^{*}=\emptyset$, and set $\lambda=\chi_{U} \in \ell^{\infty}(S)$, so that

$$
\mu\left(U^{*}\right)=\langle\mu, \lambda\rangle \quad\left(\mu \in M\left(S^{*}\right)\right),
$$

and $\lambda$ is a non-zero, continuous linear functional on $M\left(S^{*}\right)$. We shall show that $\langle\mu \square \nu, \lambda\rangle=0\left(\mu, \nu \in M\left(S^{*}\right)^{+}\right)$; this will imply that we have

$$
\operatorname{supp}(\mu \square \nu) \subset \overline{S_{[2]}^{*}} \quad\left(\mu, \nu \in M\left(S^{*}\right)\right),
$$

giving the result.

Fix $\mu, \nu \in M\left(S^{*}\right)^{+}$, and let $x \in S^{*}$. Then there is a sequence $\left(s_{n}\right)$ in $S$ such that $\lim _{n \rightarrow \infty}\left\langle s_{n}, \nu \cdot \lambda\right\rangle=\langle x, \nu \cdot \lambda\rangle$. Set

$$
U_{n}=\bigcup\left\{s_{m}^{-1} U: m \geq n\right\} \quad(n \in \mathbb{N}) .
$$

Assume towards a contradiction that $\bigcap\left\{U_{n}^{*}: n \in \mathbb{N}\right\} \neq \emptyset$, say $v \in \bigcap\left\{U_{n}^{*}: n \in \mathbb{N}\right\}$. Then $\left(s_{n_{k}} v\right) \subset \bar{U}$ for some strictly increasing sequence $\left(n_{k}\right)$ in $\mathbb{N}$. Let $u$ be an accumulation point of the sequence $\left(s_{n_{k}}\right)$. Then $u \in S^{*}$, and $u \square v \in \bar{U}$ because $R_{v}$ is continuous. Thus $u \square v \in \bar{U} \cap S_{[2]}^{*}$, a contradiction. This shows that $\bigcap\left\{U_{n}^{*}: n \in \mathbb{N}\right\}=\emptyset$.

It follows immediately that, for each $\varepsilon>0$, there exists $n_{0} \in \mathbb{N}$ such that $\nu\left(U_{n}^{*}\right)<\varepsilon\left(n \geq n_{0}\right)$. Thus

$$
\langle x \square \nu, \lambda\rangle=\langle x, \nu \cdot \lambda\rangle=\lim _{n \rightarrow \infty}\left\langle s_{n}, \nu \cdot \lambda\right\rangle=\lim _{n \rightarrow \infty} \nu\left(U_{n}^{*}\right) \leq \varepsilon .
$$

This holds for each $\varepsilon>0$, and so $\langle x \square \nu, \lambda\rangle=0$. Since $R_{\nu}$ is weak-*continuous on $M\left(S^{*}\right)$, it follows that $\langle\mu \square \nu, \lambda\rangle=0$, as required.

COROLlary 7.8. Let $S$ be an infinite, cancellative semigroup. Then $M\left(S^{*}\right)$ is not essential.

Proof. By Corollary 6.27, $\overline{S_{[2]}^{*}} \neq S^{*}$, and so, by the proposition, $\overline{M\left(S^{*}\right)^{2}} \neq M\left(S^{*}\right)$.

Corollary 7.9. Let $S$ be a semigroup such that $S^{*}$ is a subsemigroup of $\beta S$. Then $M\left(S^{*}\right)$ is a closed subalgebra of $M(\beta S)$, and $\overline{M\left(S^{*}\right)^{2}} \subset M\left(\overline{S_{[2]}^{*}}\right)$. Further, for each $\mu, \nu \in M\left(S^{*}\right)$ and each compact $K \subset S^{*} \backslash \overline{S_{[2]}^{*}}$, we have $|\mu \square \nu|(K)=0$.

A small variation of the argument in Proposition 7.7 gives the following result. 
Proposition 7.10. Let $S$ be a semigroup, and let $I$ be a closed ideal in $\beta S$ such that $M(I)$ is an ideal in $M(\beta S)$. Then

$$
\operatorname{supp}(\mu \square \nu) \subset \overline{S^{*} I} \quad\left(\mu \in M\left(S^{*}\right), \nu \in M(I)\right) .
$$

THEOREM 7.11. Let $S$ be an infinite semigroup. Then the following are equivalent:

(a) $\ell^{1}(S)$ is a dual Banach algebra

with respect to $c_{0}(S)$;

(b) $S$ is

weakly cancellative;

(c) $S^{*}$ is an ideal in $(\beta S, \square)$;

(d) $\ell^{1}\left(S^{*}\right)$ is an ideal in $\left(\ell^{1}(\beta S), \square\right)$;

(e) $M\left(S^{*}\right)$ is an ideal in $(M(\beta S), \square)$.

Proof. Set $A=\ell^{1}(S)$

(a) $\Leftrightarrow$ (b) This is Theorem 4.6.

(b) $\Leftrightarrow(\mathrm{c})$ This is Theorem 6.16(ii).

$(\mathrm{e}) \Rightarrow(\mathrm{c}) \Leftrightarrow(\mathrm{d})$ These are immediate.

(a) $\Rightarrow(\mathrm{e})$ Set $E=c_{0}(S)$. As we remarked in Chapter $2, E^{\circ}$ is a closed ideal in $\left(A^{\prime \prime}, \square\right)$. Thus $M\left(S^{*}\right)$ is a closed ideal in $M(\beta S)$.

Let $S$ be a weakly cancellative semigroup, and set $A=\ell^{1}(S)$ and $E=c_{0}(S)$. Then

$$
(M(\beta S), \square)=\left(A^{\prime \prime}, \square\right)=A \ltimes E^{\circ}=\ell^{1}(S) \ltimes M\left(S^{*}\right)
$$

and

$$
\left(\ell^{1}(\beta S), \square\right)=\ell^{1}(S) \ltimes \ell^{1}\left(S^{*}\right) .
$$

In particular this shows that $M\left(S^{*}\right)$ and $\ell^{1}\left(S^{*}\right)$ are prime ideals in $M(\beta S)$ and $\ell^{1}(\beta S)$, respectively. In the case where $\ell^{1}(S)$ is semisimple, we have $R_{M(\beta S)}=R_{M\left(S^{*}\right)}$ and $R_{\ell 1(\beta S)}=R_{\ell^{1}\left(S^{*}\right)}$.

Let $S$ be a semigroup. Recall that we have defined (in Definition 6.19) $T_{[1]}^{*}=S^{*}$ and $T_{[n+1]}^{*}=\overline{S^{*} T_{[n]}^{*}}(n \in \mathbb{N})$. In the case where $S$ is weakly cancellative, each $T_{[n]}^{*}$ is a closd ideal in $(\beta S, \square)$.

Corollary 7.12. Let $S$ be an infinite, weakly cancellative semigroup. Then $M\left(T_{[n]}^{*}\right)$ is a closed ideal in $M(\beta S)$. In particular, $M\left(\overline{S_{[2]}^{*}}\right)$ is a closed ideal in $M(\beta S)$.

Proof. This is an immediate induction from the theorem and Proposition 7.10. 
We have noted that it is not necessarily true that $T_{[3]}^{*}=\overline{S_{[3]}^{*}}$ (see Theorem 6.46). So the above result leaves open the following question.

Question Is $M\left(\overline{S_{[3]}^{*}}\right)$ a closed ideal in $M(\beta S)$ for each infinite, countable, weakly cancellative semigroup $S$ ?

Even the special case where $S=\mathbb{N}$ is open.

THEOREM 7.13. Let $S$ be an infinite, cancellative semigroup. Then there exists a non-empty subset $V$ of $S^{*}$ with $s^{-1} V t^{-1}=V(s, t \in S)$ and such that

$$
(\mu \square \nu)(\{v\})=0 \quad\left(v \in V, \mu, \nu \in M\left(S^{*}\right)\right) .
$$

Proof. Set $V=\left\{v \in S^{*}:(\mu \square \nu)(\{v\})=0 \quad\left(\mu, \nu \in M\left(S^{*}\right)\right\}\right.$. By Corollary 6.27 and Proposition 7.7, $V \neq \emptyset$.

Let $s \in S$ and $v \in V$. For each $\mu, \nu \in M\left(S^{*}\right)$, we have

$$
(\mu \square \nu)\left(\left\{s^{-1} v\right\}\right)=(\mu \square \nu \square s)(\{v\})=0,
$$

noting that $\nu \square s \in M\left(S^{*}\right)$ by Theorem 7.11. Thus $s^{-1} V \subset V$. We also have $s v \in V$ because

$$
(\mu \square \nu)(\{s v\})=\left(\mu_{s} \square \nu\right)(\{v\})=0
$$

in the notation of Proposition 7.2(ii), noting that $\mu_{s} \in M\left(S^{*}\right)$ by Theorem 7.11. Thus $V \subset s^{-1} V$, and so $s^{-1} V=V$. Similarly, we have $V t^{-1}=V(t \in S)$, and so the theorem follows.

Second duals of Rees semigroup algebras The Rees semigroup algebras were introduced in Chapter 4.

ExAmPle 7.14. Let $G$ be a group, let $m, n \in \mathbb{N}$, and consider the regular Rees matrix semigroup with zero

$S=\mathcal{M}^{o}(G, P, m, n)$ described in Chapter 3 ; here, $P=\left(a_{i j}\right) \in$ $\mathbb{M}_{n, m}\left(G^{o}\right)$ is a sandwich matrix, identified with an element of $\mathbb{M}_{n}\left(\ell^{1}(G)\right)$. We have described $\ell^{1}(S)=\left(\mathcal{M}^{o}\left(\ell^{1}(G), P, m, n\right)\right.$, $\left.\star_{P}\right)$ in Chapter 4 , and we have described the semigroup

$$
\beta S=\mathcal{M}^{o}(\beta G, P, m, n)
$$

in Example 6.18. An element of $M(\beta S)$ is now identified with an element of $\mathbb{M}_{m, n}(M(\beta G)) \cup \mathbb{C} \delta_{o}$. By using equations (4.7) and (4.8) and taking iterated limits, we see that the product in $(M(\beta S), \square)$ is given as follows. Let $\mu=\left(\mu_{i j}\right), \nu=\left(\nu_{i j}\right) \in \mathbb{M}_{m, n}(M(G))$. Then

$$
(\mu \square \nu)_{i \ell}=\sum_{(j, k) \in N(P)} \mu_{i j} \square \delta_{a_{j k}} \square \nu_{k \ell}+\sum_{(j, k) \in Z(P)} \varphi_{G}^{\prime \prime}\left(\mu_{i j}\right) \varphi_{G}^{\prime \prime}\left(\nu_{k \ell}\right) \delta_{o}
$$


and

$$
\mu \square \delta_{o}=\delta_{o} \square \mu=\left(\sum_{i=1}^{m} \sum_{j=1}^{m} \varphi_{G}^{\prime \prime}\left(\mu_{i j}\right)\right) \delta_{o} .
$$

Thus $M(\beta S) / \mathbb{C} \delta_{o}=\mathcal{M}\left(M(\beta G), P_{G}, m, n\right)$ (isometrically and isomorphically). Similar formulae describe the product in $(M(\beta S), \diamond)$.

Further we see that

$$
M\left(S^{*}\right)=\mathcal{M}\left(M\left(G^{*}\right), P, m, n\right),
$$

and so $M\left(S^{*} \cup\{o\}\right)$ is a closed, complemented ideal in $(M(\beta S), \square)$.

EXAMPLE 7.15. Let $G$ be any infinite group, take

$$
P=\left(\begin{array}{ll}
e_{G} & e_{G} \\
e_{G} & e_{G}
\end{array}\right)
$$

and set $S=\mathcal{M}^{o}(G, P, 2)$. Then $P$ is a regular sandwich matrix. Take $\mu \in M\left(G^{*}\right)$ with $\mu \neq 0$, and consider the element

$$
\Phi=\left(\begin{array}{cc}
\mu & -\mu \\
0 & 0
\end{array}\right) \in \mathbb{M}_{2}(M(G)) .
$$

It follows from (7.7) that $\Phi \square \nu=0\left(\nu \in \mathbb{M}_{2}(M(G))\right)$ and from (7.8) that $\Phi \square \delta_{o}=0$. Thus $\Phi \square \Psi=0\left(\Psi \in \ell^{1}(S)^{\prime \prime}\right)$. Similarly, we have $\Phi \diamond \Psi=0\left(\Psi \in \ell^{1}(S)^{\prime \prime}\right)$, and so $\Phi \in \mathfrak{Z}_{t}^{(\ell)}\left(\ell^{1}(S)^{\prime \prime}\right)$, whereas $\Phi \notin \ell^{1}(S)$. It follows that $\ell^{1}(S)$ is not strongly Arens irregular.

We shall see in Corollary 12.18 that $\mathcal{M}^{o}\left(\ell^{1}(G), P, n\right)$, is strongly Arens irregular whenever $P$ is invertible in $\mathbb{M}_{n}\left(\ell^{1}(G)\right)$.

Ideals on uniform ultrafilters We now investigate some properties of subspaces of $M(\beta S)$ defined in terms of the subsets $U_{\kappa}(S)$ of $S^{*}$ consisting of the $\kappa$-uniform ultrafilters.

Proposition 7.16. Let $S$ be an infinite semigroup, let $\kappa$ be an infinite cardinal with $\kappa \leq|S|$, and let $\mu \in M(\beta S)$. Then $\mu \in M\left(U_{\kappa}(S)\right)$ if and only if $\left\langle\mu, \chi_{\bar{W}}\right\rangle=0$ for each $W \in[S]^{<\kappa}$.

Proof. We have $I\left(U_{\kappa}(S)\right)=C_{0}\left(\beta S \backslash U_{\kappa}(S)\right)$, and the latter space is the closed linear span of functions of the form $\chi_{\bar{W}}$, where $W \in[S]^{<\kappa}$. The result follows.

Proposition 7.17. Let $S$ be an infinite semigroup, and let $\kappa$ be an infinite cardinal with $\kappa \leq|S|$. Then the space $M\left(\beta S \backslash U_{\kappa}(S)\right)$ is a $\|\cdot\|$-closed subalgebra of the Banach algebra $(M(\beta S), \square)$. 
Proof. We know that $L:=M\left(\beta S \backslash U_{\kappa}(S)\right)$ is a $\|\cdot\|$-closed subspace of $M(\beta S)$.

Take $\mu, \nu \in L$; we shall show that $\mu \square \nu \in L$. Since $L$ is $\|\cdot\|$-closed in $M(\beta S)$, it suffices to supose that there exist subsets $U, V \in[S]^{<\kappa}$ with supp $\mu \subset \bar{U}$ and supp $\nu \subset \bar{V}$. But now $|U V|<\kappa$ and, by Proposition 7.5 (ii), we have

$$
\operatorname{supp}(\mu \square \nu) \subset \bar{U} \square \bar{V} \subset \overline{U V} \subset \beta S \backslash U_{\kappa}(S) .
$$

Hence $\mu \square \nu \in L$, as required.

THEOREM 7.18. Let $S$ be an infinite, weakly cancellative semigroup, and let $\kappa$ be an infinite cardinal with $\kappa \leq|S|$. Then $M\left(U_{\kappa}(S)\right)$ is a closed, prime ideal in $M(\beta S)$, and

$$
M(\beta S)=M\left(\beta S \backslash U_{\kappa}(S)\right) \ltimes M\left(U_{\kappa}(S)\right)
$$

as a semidirect product.

Proof. Set $M=M(\beta S)$.

By Proposition 6.36, $U_{\kappa}(S)$ is a closed, prime ideal in $\beta S$, and so, by Proposition 7.5(i), $M\left(U_{\kappa}(S)\right)$ is a closed left ideal in $M$.

We shall prove that $\mu \square \nu \in M\left(U_{\kappa}(S)\right)$ whenever $\mu \in M\left(U_{\kappa}(S)\right)$ and $\nu \in M$, thus establishing that $M\left(U_{\kappa}(S)\right)$ is a right ideal. Since $R_{\nu}$ is continuous on $M$, it suffices to suppose that $\mu=u$, where $u \in U_{\kappa}(S)$.

Set $\nu_{1}=\nu \mid U_{\kappa}(S)$ and $\nu_{2}=\nu \mid\left(\beta S \backslash U_{\kappa}(S)\right)$, so that $\nu=\nu_{1}+\nu_{2}$. Since $u \square \nu_{1} \in M\left(U_{\kappa}(S)\right)$, it suffices to show that $u \square \nu_{2} \in M\left(U_{\kappa}(S)\right)$. By Proposition 7.17, it is further sufficient to consider the case where supp $\nu_{2} \subset \bar{V}$ for some $V \in[S]^{<\kappa}$, and so we do consider this case.

Let $W \in[S]^{<\kappa}$. We claim that there exists a set $U \in u$ such that $U V \cap W=\emptyset$. Assume towards a contradiction that this is not the case. Then, for each $U \in u$, there exists $u_{U} \in U$ and $v_{U} \in V$ with $u_{U} v_{U}=w_{U}$ for some $w_{U} \in W$. Define $T=\left\{u_{U}: U \in u\right\}$. Then $T \cap U \neq \emptyset$ for each $U \in u$, and so $T \in u$. We have $|V \times W|<\kappa$; for every $(v, w) \in V \times W$, the set $\{s \in S: s v=w\}$ is finite because $S$ is weakly right cancellative. We have

$$
W V^{-1}=\bigcup\{\{s \in S: s v=w\}:(v, w) \in V \times W\},
$$

and so $\left|W V^{-1}\right|<\kappa$. Then $T \subset W V^{-1}$, and so $|T|<\kappa$, a contradiction of the fact that $T \in u$. Thus the claim holds.

Take $s \in U \subset S \backslash W V^{-1}$. Then $\left\langle s v, \chi_{W}\right\rangle=0(v \in V)$, and so we have $\left\langle v, \chi_{\bar{W}} \cdot s\right\rangle=0(v \in V)$. It follows that $\left\langle f, \chi_{\bar{W}} \cdot s\right\rangle=0$ for each $f \in \ell^{1}(V)$, and hence

$$
\left\langle s, \nu_{2} \cdot \chi_{\bar{W}}\right\rangle=\left\langle\nu_{2}, \chi_{\bar{W}} \cdot s\right\rangle=0 .
$$


Thus $\left\langle u, \nu_{2} \cdot \chi_{\bar{W}}\right\rangle=0$, and so $\left\langle u \square \nu_{2}, \chi_{\bar{W}}\right\rangle=0$. By Proposition 7.16, we have $u \square \nu_{2} \in M\left(U_{\kappa}(S)\right)$, as required. Thus $M\left(U_{\kappa}(S)\right)$ is a closed ideal in $M(\beta S)$.

The remaining results now follow from Propositions 7.17.

Approximate identities It follows from Proposition 7.6 that $M\left(S^{*}\right)$ is a closed left ideal and, in particular, a subalgebra, of $M(\beta S)$ whenever $S$ is weakly left cancellative. Thus it is sensible in this case to ask whether or not $M\left(S^{*}\right)$ has a right (approximate) identity. The following result is immediate in the case where $S$ is cancellative, for in this case $M\left(S^{*}\right)$ is not essential by Corollary 7.8. However the result also covers some cases where $M\left(S^{*}\right)$ factors; see Example 7.32 , below, for example.

THEOREM 7.19. Let $S$ be an infinite, weakly left cancellative semigroup. Then there is no bounded net $\left(\mu_{\alpha}\right)$ in $M\left(S^{*}\right)$ such that

$$
u \square \mu_{\alpha} \rightarrow u \quad \text { weak-* } \quad\left(u \in S^{*}\right) .
$$

In particular, $\left(M\left(S^{*}\right), \square\right)$ does not have a bounded right approximate identity.

Proof. We may suppose that $S$ has an identity. We first suppose that, in addition, the semigroup $S$ is countable.

As usual, we enumerate $S$ as $\left\{s_{n}: n \in \mathbb{Z}^{+}\right\}$, with $s_{0}=e_{S}$, and we shall inductively choose a sequence $\left(t_{n}: n \in \mathbb{Z}^{+}\right)$with $t_{0}=s_{0}$. For $n \in \mathbb{Z}^{+}$, set $S_{n}=\left\{s_{0}, \ldots, s_{n}\right\}$ and $T_{n}=\left\{t_{0}, \ldots, t_{n}\right\}$ (when $t_{n}$ has been defined). Now suppose that $n \in \mathbb{Z}^{+}$, assume that $T_{n}$ has been specified, and set

$$
U_{n}=S_{n+1}^{n+1}\left(S_{n}^{n}\right)^{-1} T_{n}
$$

Then $U_{n}$ is a finite set with $U_{n} \supset\left(S_{n} \cup T_{n}\right)$. Choose $t_{n+1} \in S \backslash U_{n}$. This continues the inductive construction of the sequence $\left(t_{n}\right)$. Set $T=\left\{t_{n}: n \in \mathbb{Z}^{+}\right\}$, an infinite subset of $S$.

Let $W$ be an infinite subset of $T$, and let $k \in \mathbb{N}$. We set

$$
V_{W, k}=\bigcup\left\{\left(S_{n}^{n-k}\right)^{-1} t_{n}: t_{n} \in W, n>k\right\} .
$$

We observe that each $V_{W, k}$ is infinite and that $V_{W, j} \supset V_{W, k}(j \leq k)$. Further, if $W_{1}$ and $W_{2}$ are disjoint, infinite subsets of $T$, then it follows from the definition of $U_{n}$ that $V_{W_{1}, 1} \cap V_{W_{2}, 1}=\emptyset$. Define

$$
V_{W}=S^{*} \cap \bigcap\left\{\overline{V_{W, k}}: k \in \mathbb{N}\right\} .
$$

Since $V_{W}$ is the intersection of a decreasing sequence of non-empty, clopen subsets of $S^{*}, V_{W}$ is a non-empty, $G_{\delta^{-}}$subset of $S^{*}$. Clearly $V_{W_{1}} \cap V_{W_{2}}=\emptyset$ whenever $W_{1}$ and $W_{2}$ are disjoint, infinite subsets of $T$. 
By Proposition 5.1, there exists $R_{W} \subset S$ such that $R_{W}^{*}$ is non-empty and $R_{W}^{*} \subset V_{W}$.

We claim that $s^{-1} V_{W} \subset V_{W}(s \in S)$. Indeed, take $s=s_{j} \in S$ and $u \in s^{-1} V_{W}$. Then $s u \in V_{W}$, and so $u \in S^{*}$. Take $U \in u$. For each $k \in \mathbb{N}$ with $k>j$, there exists $t \in U$ such that $s t \in\left(S_{n}^{n-k}\right)^{-1} t_{n}$ for some $t_{n} \in W$ and $n>k$, and so $t \in V_{W, k-1}$. It follows that $U \cap V_{W, k} \neq \emptyset$ for each $k \in \mathbb{N}$, and so $u \in \overline{V_{W, k}}(k \in \mathbb{N})$. Thus $u \in V_{W}$, giving the claim.

Assume towards a contradiction that $\left(\mu_{\alpha}\right)$ is a bounded net in $M(\beta S)$ such that $(7.9)$ holds; we may suppose that $\left(\mu_{\alpha}\right) \subset M_{\mathbb{R}}\left(S^{*}\right)$. Let $\left\{W_{1}, \ldots, W_{m}\right\}$ be a partition of $T$ into infinite subsets, and then set $R_{i}=R_{W_{i}}\left(i \in \mathbb{N}_{m}\right)$. For each $i \in \mathbb{N}_{m}$, choose $u_{i} \in R_{i}^{*}$, and then choose $\alpha_{i}$ such that $\left(u_{i} \square \mu_{\alpha}\right)\left(R_{i}^{*}\right)>1 / 2$ for $\alpha>\alpha_{i}$. Choose $\alpha \geq \max \left\{\alpha_{1}, \ldots, \alpha_{m}\right\}$.

Next, for each $i \in \mathbb{N}_{m}$, choose $s_{i} \in S$ with

$$
\mu_{\alpha}\left(s_{i}^{-1} R_{i}^{*}\right)=\left\langle s_{i} \square \mu_{\alpha}, \chi_{R_{i}}\right\rangle>\frac{1}{2} .
$$

By our claim, $s_{i}^{-1} V_{W_{i}}^{*} \subset V_{W_{i}}$. Since the sets $V_{W_{1}}, \cdots, V_{W_{k}}$ are pairwise disjoint, we have

$$
\mu_{\alpha}\left(S^{*}\right) \geq \sum_{i=1}^{m} \mu_{\alpha}\left(s_{i}^{-1} R_{i}^{*}\right)>\frac{m}{2} .
$$

But this holds for each $m \in \mathbb{N}$, a contradiction of the fact that $\left(\mu_{\alpha}\right)$ is bounded.

We now consider the general case, where $S$ need not be countable.

By Proposition 3.17, there exists an infinite, countable subsemigroup $U$ of $S$ such that $U s \cap U=\emptyset(s \in S \backslash U)$, and $U$ is weakly left cancellative. Assume towards a contradiction that $\left(\mu_{\alpha}\right)$ is a bounded net in $M(\beta S)$ satisfying (7.9). Set $V=S \backslash U$, and, for each $\alpha$, set $\nu_{\alpha}=\mu_{\alpha} \mid \bar{U}$, and $\xi_{\alpha}=\mu_{\alpha} \mid \bar{V}$. Let $v \in \bar{U}$. By our condition, $\bar{U} \square \bar{V} \subset \bar{V}$, and so $\left(v \square \xi_{\alpha}\right) \mid \bar{U}=0$ for each $\alpha$. Thus $\left(\nu_{\alpha}\right)$ satisfies (7.9) with respect to the semigroup $U$, a contradiction of the special case.

We conclude that $M\left(S^{*}\right)$ does not have any bounded net satisfying (7.9).

The following result is proved in a similar way; we omit the proof. Note, however, that it is not just a symmetric version of the above theorem.

THEOREM 7.20. Let $S$ be an infinite, weakly right cancellative semigroup. Then $\left(M\left(S^{*}\right), \square\right)$ does not have a bounded left approximate identity. 
Nilpotents and radicals Let $S$ be a semigroup. We seek to determine the radical and nilpotent

elements of the two Banach algebras $\left(\ell^{1}(S), \square\right)$ and $(M(\beta S), \square)$.

Let $S$ be a semigroup, and set

$$
J_{1}=\{\mu \in M(\beta S): s \cdot \mu=\mu(s \in S), \mu(\beta S)=0\} .
$$

It is easily seen that $J_{1}$ is a closed, nilpotent ideal

of index 2 in $M(\beta S)$, and so $J_{1} \subset R_{M(\beta S)}$.

The standard definitions of the set $\mathfrak{L}(S)$ of left-invariant means on a semi-group $S$, of left-amenable semigroups, and of amenable groups are recalled in Definition 9.1, below. Granirer [60] proved that $R_{M(\beta S)}$ is infinite-dimensional whenever $S$ is an infinite, left-cancellative, leftamenable semigroup. The following proposition extends this result: clause (i) follows from [116, Theorem (7.31)(ii)(b)] and clause (ii) follows from $[\mathbf{8 7}$, Corollary 4.5] (see also $[\mathbf{1 3 6}]$ ).

Proposition 7.21. Let $S$ be an infinite, left-amenable semigroup.

(i) Suppose that $S$ is left cancellative. Then $\operatorname{dim} R_{M(\beta S)} \geq 2^{\mathfrak{c}}$.

(ii) Suppose that $S$ has no finite left ideal groups or that there are infinitely many finite left ideal groups. Then the space $R_{M(\beta S)}$ is infinitedimensional.

Let $G$ be an infinite and amenable or locally compact and nondiscrete group. Then $R_{M(\beta S)}$ is not norm-separable [63]. It seems to be an open question for each non-amenable group $G$ whether or not $M(\beta G)$ is semisimple.

There is a generalization of the above result, which we give just in the case where $S=\mathbb{Z}$. Set $A=\ell^{1}(\mathbb{Z})$ and $M=M(\beta \mathbb{Z})$.

For $\zeta \in \mathbb{T}$, regard $\zeta$ as an element of $\Psi_{\beta \mathbb{Z}}$, so that $\zeta$ gives a character on $A$; indeed, $\left\langle\delta_{m}, \zeta\right\rangle=\zeta^{m}(m \in \mathbb{N})$. The second dual of this character is the character $\mu \mapsto\langle\mu, \zeta\rangle$ on $M$. For $n \in \mathbb{N}$ and $r \in \mathbb{Z}, \chi_{n, r}$ is the characteristic function of the set $n \mathbb{Z}+r$; we denote by $\chi_{n, r, \zeta}$ the function whose value at $k \in \mathbb{Z}$ is $\zeta^{k} \chi_{n, r}(k)$, so that $\chi_{n, r, \zeta} \in \ell^{\infty}(\mathbb{Z})$. We note that

$$
\delta_{r} \cdot \chi_{n, s, \zeta}=\chi_{n, s, \zeta} \cdot \delta_{r}=\zeta^{r} \chi_{n, s-r, \zeta} \quad(n \in \mathbb{N}, r, s \in \mathbb{Z}, \zeta \in \mathbb{T}) .
$$

Now define

$$
J_{n, \zeta}=\left\{\mu \in M: n \square \mu=\zeta^{n} \mu,\left\langle\mu, \chi_{n, r, \zeta}\right\rangle=0\left(r \in \mathbb{N}_{n}\right)\right\}
$$

Clearly $J_{n, \zeta}$ is a $\sigma\left(M, A^{\prime}\right)$-closed linear subspace of $M$, and we see easily that $J_{n, \zeta}$ is an ideal in $M$ of index 2 . Let $\zeta \in \mathbb{T}$, and set

$$
J_{\zeta}=\overline{\bigcup\left\{J_{m, \zeta}: m \in \mathbb{N}\right\}} \text {. }
$$


Since $J_{m, \zeta} \cup J_{n, \zeta} \subset J_{m n, \zeta}(m, n \in \mathbb{N})$, it follows that $J_{\zeta}$ is a closed, nilpotent ideal of index 2 in $M$, and so $J_{\zeta} \subset R_{M}$. Indeed, the family $\left\{J_{2^{m}, \zeta}: m \in \mathbb{N}\right\}$ is an ascending chain of distinct closed ideals in $M$. Thus there is an element $\mu \in J_{\zeta}$ such that $\mu \notin J_{m, \zeta}$ for any $m \in \mathbb{N}$. Finally, we set

$$
J=\varlimsup \operatorname{lin}\left\{J_{\zeta}: \zeta \in \mathbb{T}\right\} .
$$

Then $J$ is a closed, nilpotent ideal of index 2 in $M$. We have obtained the following result.

TheOREM 7.22. The set $J$ is a closed, nilpotent ideal of index 2 in the Banach algebra $(M(\beta \mathbb{Z}), \square)$, and so $J \subset R_{M(\beta \mathbb{Z})}$.

These remarks were first established by Civin and Yood

$[\mathbf{1 6}$, Theorem 3.5] in the special case where $\zeta=1$.

We now exhibit some nilpotent

elements of our algebras. We require a preliminary lemma, suggested by George Willis.

Lemma 7.23. (i) For each $n \in \mathbb{N}$, the algebra $\ell^{1}\left(\mathbb{F}_{2}\right)$ contains a non-zero nilpotent element of index $n+1$.

(ii) The algebra $\ell^{1}\left(\mathbb{F}_{2}\right)$ contains a quasi-nilpotent

element which is not nilpotent.

Proof. (i) Let $n \in \mathbb{N}$.

First we consider the commutative group algebra $\ell^{1}(\mathbb{Z})$. The Fourier transform of $\ell^{1}(\mathbb{Z})$ is defined to be the Banach function algebra $A(\mathbb{T})$ on $\mathbb{T}$; since $A(\mathbb{T})$ contains all functions on $\mathbb{T}$ that have 2 continuous derivatives, it is clear that there are non-zero functions $h_{1}, \ldots, h_{n+1}$ in $A(\mathbb{T})$ such that $h_{i} h_{j}=0$ for $i, j \in \mathbb{N}_{n+1}$ and $i \neq j$. Thus there exist non-zero functions $f_{1}, \ldots, f_{n+1}$ in $\ell^{1}(\mathbb{Z})$ such that $f_{i} \star f_{j}=0$ whenever $i, j \in \mathbb{N}_{n+1}$ and $i \neq j$. Clearly we have $f_{i} \star f_{i} \neq 0\left(i \in \mathbb{N}_{n+1}\right)$. We may suppose that $\left\|f_{i}\right\|_{1}=1\left(i \in \mathbb{N}_{n+1}\right)$.

Set $A=\ell^{1}\left(\mathbb{F}_{2}\right)$. We denote the two generators of the group $\mathbb{F}_{2}$ by $a$ and $b$; we identify $\mathbb{Z}$ with the subgroup $\left\{a^{n}: n \in \mathbb{Z}\right\}$ of $\mathbb{F}_{2}$, and thus regard $\ell^{1}(\mathbb{Z})$ as a closed subalgebra of $A$. For convenience, we write $f b g$ for the element $f \star \delta_{b} \star g$ when $f, g \in A$; we similarly abbreviate other products in $A$.

Define

$$
f=\sum_{j=1}^{n} f_{j} b f_{j+1} \quad \text { in } \quad A .
$$

By calculation, we see that $f^{2}=\sum_{j=1}^{n-1} f_{j} b f_{j+1}^{2} b f_{j+2}$, and, continuing, that

$$
f^{n}=f_{1} b f_{2}^{2} b f_{3}^{2} b \cdots b f_{n}^{2} b f_{n+1} .
$$


It follows that $f^{n+1}=0$.

We claim that $f^{n} \neq 0$, which will imply the result. Indeed, take $k \in \mathbb{N}$ with $k \geq 2$, let $g_{1}, \ldots, g_{k}$ be non-zero elements of $\ell^{1}(\mathbb{Z})$, say with $\left\|g_{j}\right\|_{1}=1\left(j \in \mathbb{N}_{k}\right)$, and set $g=g_{1} b g_{2} b \cdots b g_{k}$. Then the support of $g$ is a family of words in $\mathbb{F}_{2}$ of the form

$$
a^{m_{1}} b a^{m_{2}} b \cdots b a^{m_{k}}
$$

where $m_{1}, \ldots, m_{k} \in \mathbb{Z}$ and $a^{m_{j}} \in \operatorname{supp} g_{j}\left(j \in \mathbb{N}_{k}\right)$. Now two words of the form $a^{m_{1}} b a^{m_{2}} b \cdots b a^{m_{k}}$ and $a^{n_{1}} b a^{n_{2}} b \cdots b a^{n_{k}}$ in $\mathbb{F}_{2}$ are equal if and only if $m_{j}=n_{j}\left(j \in \mathbb{N}_{k}\right)$, and so there can be 'no cancellation' when the product $g_{1} b g_{2} b \cdots b g_{k}$ is formed. This shows that $\|g\|_{1}=1$, and so $g \neq 0$. Hence (i) is established.

(ii) The above proof shows that, for each $n \in \mathbb{N}$, there exist elements $f_{1, n}, \ldots, f_{n+1, n}$ in $\ell^{1}(\mathbb{Z})$ with $\left\|f_{i, n}\right\|_{1}=1\left(i \in \mathbb{N}_{n+1}\right)$ and such that the element $F_{n}:=\sum_{j=1}^{n} f_{j, n} b f_{j+1, n}$ has the property that $\left\|F_{n}^{n}\right\|_{1}=1$ and $F_{n}^{n+1}=0$ in $\ell^{1}\left(\mathbb{F}_{2}\right)$. We have $\left\|F_{n}\right\|_{1} \leq n$. By making a suitable choice of elements in $A(\mathbb{T})$, we may also suppose that $f_{i, m} f_{j, n}=0$ whenever $m \neq n$ and for all appropriate $i, j$, and so $F_{m} F_{n}=0$ in $A$ whenever $m \neq n$.

Now choose constants $\alpha_{k}>0$ such that $\alpha_{k}<2^{-k}(k \in \mathbb{N})$ and

$$
\sum_{n=k+1}^{\infty} n^{k} \alpha_{n}^{k}<\alpha_{k}^{k} \quad(k \in \mathbb{N}),
$$

and set $F=\sum_{n=1}^{\infty} \alpha_{n} F_{n}$, so that $F \in A$ because $\sum_{n=1}^{\infty} n\left|\alpha_{n}\right|<\infty$. Then

$$
F^{k}=\sum_{n=k}^{\infty} \alpha_{n}^{k} F_{n}^{k} \quad(k \in \mathbb{N}),
$$

and so $F^{k} \neq 0(k \in \mathbb{N})$, whence $F$ is not nilpotent. However, we see that $\left\|F^{k}\right\|_{1} \leq 2 \cdot 2^{-k^{2}}$, and so $\nu_{A}(F)=0$; thus $F$ is quasi-nilpotent.

THEOREM 7.24. Let $S$ be an infinite semigroup which is weakly left cancellative and nearly right cancellative, and set $L=\ell^{1}(\beta S)$ and $M=M(\beta S)$. Then the Banach algebra $L$ contains a nilpotent

element of index $n+1$ for each $n \in \mathbb{N}$. Further,

$$
\{0\} \neq N_{L} \subsetneq Q_{L} \subsetneq L \quad \text { and } \quad\{0\} \neq N_{M} \subsetneq Q_{M} \subsetneq M .
$$

ProOF. By Proposition 6.25, $\ell^{1}\left(S^{*}\right)$ contains an isometric and isomorphic copy of $\ell^{1}\left(\mathbb{F}_{2}\right)$ as a closed subalgebra, and so it follows from Proposition 7.23 that $\{0\} \neq N_{L} \subsetneq Q_{L}$. Let $s \in \mathbb{Z}^{*}$. Then $\left\|s^{n}\right\|_{1}=1 \quad(n \in \mathbb{N})$, and so $\nu_{L}(s)=1$. Thus $s \notin Q_{L}$, and hence $Q_{L} \subsetneq L$. 
We have not been able to determine whether or not $\ell^{1}(\beta \mathbb{Z})$ is semisimple, and whether or not the radical of $M(\beta \mathbb{Z})$ is equal to the ideal $J$ specified in Theorem 7.22 , above.

The semi-character space of $\beta S$ Let $S$ be a semigroup, and set $A=\ell^{1}(S)$. We shall now consider the character spaces of $\left(\ell^{1}(\beta S), \square\right)$ and $A^{\prime \prime}=(M(\beta S), \square)$; for the algebra $\ell^{1}(\beta S)$, we must find the semicharacters on $(\beta S, \square)$.

Recall that the spaces of semi-characters and characters on a semigroup $S$ are denoted by $\Phi_{S}$ and $\Psi_{S}$, respectively. We have remarked in Chapter 3 that $\left(\Phi_{S} \cup\{0\}, \cdot\right)$ and $\left(\Psi_{S}, \cdot\right)$ are unital, abelian, compact topological semigroups;

the former is the dual semigroup to $S$. Recall also that $\Phi_{G}=\Psi_{G}=$ $\Gamma$, the dual group of $G$, when $G$ is an abelian group.

Definition 7.25. Let $S$ be a semigroup, and let $\varphi \in \Phi_{\beta S}$. Then $\varphi$ is a continuous semi-character

if it is continuous with respect to the topology of $\beta S$.

Here are some examples of continuous semi-characters on $(\beta S, \square)$. For $\zeta \in \Phi_{S}$, we denote by $\widehat{\zeta}$ the element of $C(\beta \mathbb{Z})$ that is the extension of the function $s \mapsto \zeta(s)$ on $S$. Then the map

$$
\widehat{\zeta}: u \mapsto \widehat{\zeta}(u), \quad \beta S \rightarrow \overline{\mathbb{D}},
$$

is a continuous semi-character on $\beta S$; in the case where $\zeta \in \Psi_{S}$, also $\widehat{\zeta}$ is a continuous character on $\beta S$. Set

$$
\iota: \zeta \mapsto \widehat{\zeta}, \quad \Phi_{S} \rightarrow \Phi_{\beta S} .
$$

Then $\iota$ is an injection.

Let $T$ be a subsemigroup of $\beta S$ such that $\beta S \backslash T$ is an ideal, and hence a prime ideal, of $\beta S$, and let $\varphi$ be a semi-character on $(\beta S, \square)$. Then $\varphi \cdot \chi_{T}$ is also a semi-character on $(\beta S, \square)$. For example, suppose that $S$ is weakly cancellative, and take $T=S$; we obtain the family of semi-characters that correspond to the semi-character space $\Phi_{S}$ of $\ell^{1}(S)$ (but in a different way from that specified in equation (7.10)). If $K=K(\beta S)$ were a prime ideal in $\beta S$, we would obtain an interesting new semi-character. Let $G$ be a countable, abelian group, let $F$ be an almost left disjoint subset of $G$, and set $I=\beta G \backslash\left\langle G \square F^{*}\right\rangle$ in the notation of Proposition $6.34(\mathrm{v})$. Then our result shows that $I$ is a prime ideal

in $\beta G$, and so this is another way of generating semi-characters on $(\beta G, \square)$ 
For example, set $S=(\mathbb{Z},+)$, so that $\Phi_{S}=\Psi_{S}=\mathbb{T}$. We regard $\mathbb{Q} / \mathbb{Z}$ and $\mathbb{R} / \mathbb{Z}$ as subsets of $\iota(\mathbb{T})$ by identifying $x \in \mathbb{R} / \mathbb{Z}$ with the element $\iota(\zeta)$, where $\zeta=\exp (2 \pi \mathrm{i} x)$. Then $\iota(\zeta) \in \Psi_{\beta \mathbb{Z}}$. However, there are many other semi-characters.

Proposition 7.26. Let $G$ be an abelian group with dual group $\Gamma$.

(i) Each continuous semi-character on $\beta G$ is a character, and has the form $\iota(\zeta)$ for some $\zeta \in \Gamma$.

(ii) For each $\zeta \in \Gamma$, the map $\mu \mapsto\langle\mu, \iota(\zeta)\rangle$ is a character on $M(\beta G)$.

Proof. (i) This is immediate.

(ii) Set $M=M(\beta G)$. Clearly the map $\varphi_{\zeta}: \mu \rightarrow\langle\mu, \zeta\rangle$ is in $M^{\prime}$. For $\nu \in M$ and $\zeta \in \Gamma$, we have $\nu \cdot \zeta=\langle\nu, \zeta\rangle \zeta$. Now take $\mu, \nu \in M$ and $\zeta \in \Gamma$. Then

$$
\langle\mu \square \nu, \zeta\rangle=\langle\mu, \nu \cdot \zeta\rangle=\langle\mu, \zeta\rangle\langle\nu, \zeta\rangle,
$$

and so $\varphi_{\zeta} \in \Phi_{M}$.

Let $S$ be a semigroup. We denote the topology on $\Phi_{\beta S}$ of pointwise convergence on $\beta S$ by $\tau$, and we identify $\Phi_{S}$ with $\iota\left(\Phi_{S}\right)$, and so regard $\Phi_{S}$ as a subsemigroup of $\Psi_{\beta S} \subset \Phi_{\beta S}$. We are interested in the relative topology $\tau$ on $\Phi_{S}$. The usual topology on $\Phi_{S}$ is denoted by $d$; certainly we have $d \subset \tau$. The closure of $\iota\left(\Phi_{S}\right)$ in $\left(\Psi_{\beta S}, \tau\right)$ is denoted by $\bar{\Phi}_{S}$.

An early work on the space analogous to $\Phi_{G}$ for a locally compact abelian group $G$ is by Glicksberg [58]; we are grateful to Salvador Hernàndez Muñoz for this and other references. The main result of Glicksberg is that a subset of $G$ which is $\tau$-compact is already compact in $G$; the proof relies strongly on a theorem of Grothendieck [69].

TheOREM 7.27. Let $G$ be an infinite abelian group.

(i) There are characters on $\beta G$ that are not continuous, and the map $\iota:\left(\Phi_{G}, d\right) \rightarrow\left(\Phi_{\beta G}, \tau\right)$ is not sequentially continuous.

(ii) The map $R: \varphi \mapsto \varphi \mid \ell^{1}(\beta G), \Phi_{M(\beta G)} \rightarrow \Phi_{\beta G}$, is a continuous surjection.

Proof. Set $M=M(\beta G)$ and $L=\ell^{1}(\beta G)$.

(i) This is a consequence of [58, Corollary 2.5]. Here is an elementary proof in the case where $G=\mathbb{Z}$ (and $\left.\Phi_{\mathbb{Z}}=\mathbb{T}\right)$.

For $k \in \mathbb{N}$, set $\alpha_{k}=1 \cdot 3 \cdots(2 k-1)$ (so that $\left.\alpha_{k+1}=(2 k+1) \alpha_{k}\right)$, and set $\zeta_{k}=\exp \left(\mathrm{i} \pi / \alpha_{k}\right)$, so that $\zeta_{k} \in \mathbb{T}$ and $\lim _{k} \zeta_{k}=1$ in $\mathbb{T}$. Then $\lim _{k} \zeta_{k}^{n}=1$ for each $n \in \mathbb{Z}$. The sequence $\left(\iota\left(\zeta_{k}\right): k \in \mathbb{N}\right)$ consists of continuous characters in the compact space $\Psi_{\beta \mathbb{Z}}$, and so has an accumulation point, say $\varphi \in \Psi_{\beta \mathbb{Z}}$. Clearly we have $\varphi|\mathbb{Z}=1| \mathbb{Z}$. 
For $k \in \mathbb{N}$, set $F_{k}=\alpha_{k}(2 \mathbb{N}+1)$, an infinite subset of $\mathbb{N}$; the closure of $F_{k}$ in $\beta \mathbb{Z}$ is $\overline{F_{k}}$. For each $n \in F_{k}$, we have $\zeta_{k}^{n}=-1$, and this implies that $\zeta_{k}(s)=-1\left(s \in \overline{F_{k}}\right)$. Take $n \in F_{k+1}$, say $n=m \alpha_{k+1}$ for some $m \in 2 \mathbb{N}+1$. Then $n=(2 k+1) m \alpha_{k} \in F_{k}$. Thus $F_{k+1} \subset F_{k}$, and so $\overline{F_{k+1}} \subset \overline{F_{k}}$. Since $\beta \mathbb{Z}$ is compact, there exists $s_{0} \in \bigcap\left\{\overline{F_{k}}: k \in \mathbb{N}\right\}$, and we have $\varphi\left(s_{0}\right)=-1$. Hence $\varphi$ is not continuous on $\beta \mathbb{Z}$, and so $\varphi$ is not a continuous character.

This also shows that the sequence $\left(\iota\left(\zeta_{k}\right)\right)$ is not convergent in $\Phi_{\beta \mathbb{Z}}$, and so the map $\iota:(\mathbb{T}, d) \rightarrow\left(\Phi_{\beta \mathbb{Z}}, \tau\right)$ is not sequentially continuous.

(ii) This is immediate from Proposition 2.21(ii).

TheOREM 7.28. Let $G$ be a countable, infinite abelian group with dual group $\Gamma$.

(i) There are $2^{2^{c}}$ semi-characters which are not characters on $\beta G$.

(ii) There are $2^{2^{\mathrm{c}}}$ characters on $\beta G$, each of which belongs to $\overline{\iota(\Gamma)}$.

Proof. (i) Let $F$ be an almost left disjoint subset of $G$. We define $\varphi(s)=1(s \in G)$ and define $\varphi(u)$ arbitrarily in $\mathbb{D}$ for $u \in F^{*}$. By Proposition 6.34(iv), $\left\langle F^{*}\right\rangle$ is a free semigroup, and so $\varphi$ can be extended to a semigroup homomorphism $\varphi:\left\langle G \square F^{*}\right\rangle \rightarrow \mathbb{D}$.

Set $U=\beta G \backslash\left\langle G \square F^{*}\right\rangle$. By Proposition 6.34(v), $U$ is a prime ideal in $\beta G$. Set $\varphi(u)=0(u \in U)$. Then we have extended $\varphi$ to a semi-character on $\beta G$; the extension is not a character.

By Proposition 6.34(i), $\left|F^{*}\right|=2^{\mathfrak{c}}$, and so the result follows.

(ii) This is contained in both $[\mathbf{7 1}]$ and $[\mathbf{1 3 2}]$.

In fact, the argument for (ii), above, shows that there exists an element $\varphi \in \Psi_{\beta G}$ whose range is all of $\overline{\mathbb{D}}$.

Thus in the case where $S$ is the semigroup $(\beta \mathbb{Z}, \square)$, we have

$$
\iota(\mathbb{T}) \subsetneq \Psi_{S} \subsetneq \Phi_{S} \quad \text { and } \quad|\overline{\iota(\mathbb{T})}|=\left|\Phi_{S} \backslash \Psi_{S}\right|=2^{2^{c}} .
$$

For further results on the space $\left(\overline{\Phi_{G}}, \tau\right)$, see $[\mathbf{1 3 2}]$. We conclude with a summary for the specific case where $G$ is equal to $\mathbb{Z}$; all the results follow from more general statements in [58].

TheOREM 7.29. The topology $\tau$ on $\mathbb{T}$ is neither discrete nor metrizable; the only convergent sequences in $(\mathbb{T}, \tau)$ are eventually constant; the only compact subsets of $(\mathbb{T}, \tau)$ are finite; the space $(\mathbb{T}, \tau)$ is not locally compact.

The main question that we have left open is the following. 
Question Is $\mathbb{T} \tau$-dense in $\Psi_{\beta \mathbb{Z}}$ ? If this is not the case, is the space $\Psi_{\beta \mathbb{Z}}$ connected?

Examples Let us discuss the topological centres and radicals of some examples.

ExAmPLE 7.30. Let $S$ be a set with $|S| \geq 2$, and consider the right zero semigroup

on $S$. Then $u \square v=u \diamond v=v(u, v \in \beta S)$, and so $S$ is Arens regular. Also we have

$$
\mu \square \nu=\mu \diamond \nu=\langle\mu, 1\rangle \nu \quad(\mu, \nu \in M(\beta S)),
$$

and so $\ell^{1}(S)$ is Arens regular, as noted in [92].

By Proposition 2.13, $R_{M(\beta S)}=\{\mu \in M(\beta S):\langle\mu, 1\rangle=0\}$, and so $M(\beta S)$ is not semisimple.

EXAMPLE 7.31. Let $S=\mathbb{Z}^{2}$ be the semigroup specified in Example 3.32 , so that $S$ is left cancellative, but not weakly right cancellative.

Set $U=\{0\} \times \mathbb{Z}$, so that $U$ is a subsemigroup of $S$, and take $u \in U^{*}$. Then we check easily that $u \in \mathfrak{Z}_{t}^{(\ell)}(\beta S)$ and $u \in \mathfrak{Z}_{t}^{(r)}(\beta S)$, and so $S$ is neither left nor right strongly Arens irregular. Since $\mathbb{Z} \times\{0\}$ is a subgroup of $S$ and $\ell^{1}(\mathbb{Z})$ is not Arens regular, $\ell^{1}(S)$ is not Arens regular.

ExAmple 7.32. Let $S$ be the semigroup $\mathbb{N}_{\vee}$ described in Example 4.9. Then $S$ is weakly cancellative and, by Theorem $7.11, I=M\left(S^{*}\right)$ is a closed ideal in $(M(\beta S), \square)$.

Let $u, v \in S^{*}$. Then $u \square v=v$ and $u \diamond v=u$, so that $\left(S^{*}, \square\right)$ is a right zero semigroup. Clearly $\mathfrak{Z}_{t}^{(\ell)}(\beta S)=\mathfrak{Z}_{t}^{(r)}(\beta S)=S$, and so $S$ is strongly Arens irregular. We have

$$
\begin{aligned}
& \mu \square \nu=\langle\mu, 1\rangle \nu \quad(\mu \in M(\beta S), \nu \in I), \\
& \mu \diamond \nu=\langle\nu, 1\rangle \mu \quad(\mu \in I, \nu \in M(\beta S)) .
\end{aligned}
$$

Thus the closed ideal $M\left(S^{*}\right)$ factors, despite the fact that, by Theorems 7.19 and $7.20, M\left(S^{*}\right)$ has neither a bounded left approximate identity nor a bounded right approximate identity. Set

$$
\varphi(\mu)=\langle\mu, 1\rangle \quad(\mu \in M(\beta S)),
$$

so that $\varphi$ is a character on $M(\beta S)$ and $\varphi \mid I$ is the unique character on $I$.

Let $\mu \in M\left(S^{*}\right)$ be such that $\mu \square a=\mu \diamond a$ and $\mu \square b=\mu \diamond b$ for any two specified distinct points $a, b \in S^{*}$. Then clearly $\mu=0$. Thus $\ell^{1}(S)$ is strongly Arens irregular. 
By Example 4.9, $\ell^{1}(S)$ is semisimple, and so $R_{M(\beta S)}=R_{I}$ and $R_{\ell^{1}(\beta S)}=R_{\ell^{1}\left(S^{*}\right)}$. By Proposition 2.13, $R_{I}=N_{I}=Q_{I}=(\operatorname{ker} \varphi) \cap I$, and so

$$
R_{M(\beta S)}=\left\{\mu \in M\left(S^{*}\right):\langle\mu, 1\rangle=0\right\} .
$$

Similarly, $R_{\ell^{1}(\beta S)}=\left\{\mu \in \ell^{1}\left(S^{*}\right):\langle\mu, 1\rangle=0\right\}$. It follows that $M(\beta S)$ and $\ell^{1}(\beta S)$ are not semisimple.

ExAmple 7.33. Let $S$ be the semigroup $\mathbb{N}_{\wedge}$ described in Example 4.10. Then $S$ is abelian, but not weakly cancellative, so that $M\left(S^{*}\right)$ is not an ideal in $(M(\beta S), \square)$.

Let $u, v \in S^{*}$. Then $u \square v=u$ and $u \diamond v=v$, so that $\left(S^{*}, \square\right)$ is a left zero semigroup. Clearly $\mathfrak{Z}_{t}^{(\ell)}(\beta S)=\mathfrak{Z}_{t}^{(r)}(\beta S)=S$, and so $S$ is strongly Arens irregular. We have

$$
\begin{aligned}
& \mu \square \nu=\langle\nu, 1\rangle \mu \quad\left(\mu \in M(\beta S), \nu \in M\left(S^{*}\right)\right), \\
& \mu \diamond \nu=\langle\mu, 1\rangle \nu \quad\left(\mu \in M\left(S^{*}\right), \nu \in M(\beta S)\right),
\end{aligned}
$$

so that $M\left(S^{*}\right)$ is a closed subalgebra of $M(\beta S)$ and $M\left(S^{*}\right)$ factors. Also,

$$
f \cdot \mu=\mu \cdot f=\langle\mu, 1\rangle f \quad\left(f \in \ell^{1}(S), \mu \in M\left(S^{*}\right)\right) .
$$

It follows that $\ell^{1}(S)$ is a closed ideal in $M(\beta S)$, and so we have the decomposition $M(\beta S)=M\left(S^{*}\right) \ltimes \ell^{1}(S)$.

Let $\mu \in M\left(S^{*}\right)$ be such that $\mu \square a=\mu \diamond a$ and $\mu \square b=\mu \diamond b$ for any two specified distinct points $a, b \in S^{*}$. Then clearly $\mu=0$. Thus $\ell^{1}(S)$ is strongly Arens irregular.

EXAMPLE 7.34. Let $S$ be the abelian, countable, weakly cancellative semigroup $\mathbb{N} \times\{0,1\}$ of Example 3.33, and set $A=\ell^{1}(S)$. Then $\beta S$ is identified as a set with $\beta \mathbb{N} \times\{0,1\}$ and

$$
(u, i) \square(v, j)=(u \square v, 0), \quad(u, i) \diamond(v, j)=(u \diamond v, 0)
$$

for each $u, v \in \beta \mathbb{N}$ and $i, j \in\{0,1\}$. Further, $M(\beta S)$ can be identified with $M(\beta \mathbb{N}) \times\{0,1\}$, and

$$
(\mu, i) \square(\nu, j)=(\mu \square \nu, 0), \quad(\mu, i) \diamond(\nu, j)=(u \diamond v, 0)
$$

for each $\mu, \nu \in M(\beta S)$ and $i, j \in\{0,1\}$. Now choose $u \in \mathbb{N}^{*}$. For each $(\nu, j) \in \beta S$, we have

$$
((u, 0)-(u, 1)) \square(\nu, j)=(u \square \nu, 0)-(u \square \nu, 0)=(0,0)
$$

and, similarly, $((u, 0)-(u, 1)) \diamond(\nu, j)=(0,0)$. This shows that we have $(u, 0)-(u, 1) \in \mathfrak{Z}_{t}^{(\ell)}\left(A^{\prime \prime}\right)$. Clearly $(u, 0)-(u, 1) \notin A$, and so $A$ is not strongly Arens irregular.

It is also clear that $A$ is not Arens regular. 
We shall see in Example 12.21 that, nevertheless, $S$ itself is strongly Arens irregular. 



\section{CHAPTER 8}

\section{Related spaces and compactifications}

In this chapter, we shall consider introverted $C^{*}$-subalgebras of Banach modules $\ell^{\infty}(S)$ for a semigroup $S$ and of $L^{\infty}(G)$ for a locally compact group $G$. In particular, we shall define the spaces $W A P(S)$ and $A P(S)$ on a semigroup $S$, and the four spaces $W A P(G), A P(G), L U C(G)$, and $R U C(G)$ on a locally compact group $G$, together with their character spaces, which are compactifications of $S$ and $G$, respectively.

It is possible to find a framework of semi-topological semigroups with certain extra properties that subsumes these two similar theories, but we have chosen not to follow this route.

Submodules of $\ell^{\infty}(S)$ Let $S$ be a semigroup, and then let $X$ be an introverted $C^{*}$-subalgebra (see Definition 7.3) of $\ell^{\infty}(S)$ such that

$c_{0}(S) \subset X$, so that $\left(X^{\prime}, \square\right)$ and $\left(X^{\prime}, \diamond\right)$ are Banach algebras. The character space of $X$ is $\Phi_{X}$. As in Chapter 7 , the space $\left(\Phi_{X}, \square\right)$ is a compact, right topological semigroup,

the map $\gamma_{X}: S \rightarrow \Phi_{X}$ is an injection, and $\Phi_{X}$ is a compactification of $S$; we regard $S$ as a subsemigroup of $\left(\Phi_{X}, \square\right)$ and of $\left(\Phi_{X}, \diamond\right)$. As in equation (7.1), there is a continuous semigroup homomorphism $\pi_{X}:(\beta S, \square) \rightarrow\left(\Phi_{X}, \square\right)$, and this map is the identity on $S$.

DeFinition 8.1. Let $S$ be a semigroup, and let $X$ be an introverted $C^{*}$-subalgebra of $\ell^{\infty}(S)$ such that $c_{0}(S) \subset X$. Then

$$
\Phi_{X}^{*}=\Phi_{X} \backslash S, \quad\left(\Phi_{X}^{*}\right)_{[2]}=\left\{u \square v: u, v \in \Phi_{X}^{*}\right\} .
$$

It is clear that $\Phi_{X}^{*}$ is an ideal or subalgebra of $\Phi_{X}$ if and only if $S^{*}$ is an ideal or subalgebra, respectively, of $\beta S$.

Let $S$ be a weakly cancellative semigroup, and set $A=\ell^{1}(S)$ and $E=c_{0}(S)$, as before. Let $X$ be an introverted $C^{*}$-subalgebra of $\ell^{\infty}(S)$ with $E \subset X$, so that $E^{\circ} \mid X$ is a closed ideal in $X^{\prime}$. We denote the quotient map $\left(X^{\prime}, \square\right) \rightarrow\left(X^{\prime}, \square\right) /\left(E^{\circ} \mid X\right)=A$ by $q_{X, S}$, so that $q_{X, S}$ is the dual of the canonical embedding of $E$ in $X$, and we write $q_{S}$ in the special case where $X=A^{\prime}$. Clearly

$$
q_{X, S}\left(R_{\left(X^{\prime}, \square\right)}\right)=R_{A},
$$


and so $R_{\left(A^{\prime \prime}, \square\right)} \cap A=R_{A}$. Let $\varphi_{S}$ be the augmentation character on $A$, and define

$$
\widetilde{\varphi}_{X, S}=\varphi_{S} \circ q_{X, S},
$$

with $\widetilde{\varphi}_{S}=\varphi_{S} \circ q_{S}$. Then $\widetilde{\varphi}_{X, S}$ is a character on $\left(X^{\prime}, \square\right)$, called the discrete augmentation character on $X^{\prime}$. The discrete augmentation character on $A^{\prime \prime}$

is different from the character $\varphi_{S}^{\prime \prime}$ on $A^{\prime \prime}$. In fact,

$$
\widetilde{\varphi}_{S}((f, \mu))=\varphi_{S}(f) \quad\left(f \in A, \mu \in M\left(S^{*}\right)\right) .
$$

We now give some examples of such introverted $C^{*}$-subalgebras.

DeFinition 8.2. Let $S$ be a semigroup, and let $\lambda \in \ell^{\infty}(S)=\ell^{1}(S)^{\prime}$. Then

$$
L O(\lambda)=\{\lambda \cdot t: t \in S\} .
$$

The functional $\lambda$ is weakly almost periodic (respectively, almost periodic) if the set $L O(\lambda)$ is relatively compact in the weak (respectively, $\|\cdot\|-)$ topology on $\ell^{\infty}(S)$.

The spaces of these functionals are denoted by $W A P(S)$ and $A P(S)$, respectively.

Let $S$ be a semigroup, and set $A=\ell^{1}(S)$. The spaces $W A P(S)$ and $A P(S)$ are easily identified with $W A P(A)$ and $A P(A)$, respectively, so that they are each introverted

subspaces of $\ell^{\infty}(S)$. Clearly each of $A P(S)$ and $W A P(S)$ is a $C^{*}$ subalgebra of $\ell^{\infty}(S)$, and

$$
A P(S) \subset W A P(S) \subset \ell^{\infty}(S) .
$$

Thus $W A P(A)$ and $A P(A)$ are introverted $C^{*}$-subalgebras of $\ell^{\infty}(S)$.

An example of a semigroup $S$ with $A P(S) \subsetneq W A P(S)=\ell^{\infty}(S)$ is given in [104, Theorem 3].

Let $S$ be an infinite semigroup. Then $\left(\Phi_{W A P(S)}, \square\right)$ coincides with the compact, left topological semigroup $\left(\Phi_{W A P(S)}, \diamond\right)$, and so the semigroup $\left(\Phi_{W A P(S)}, \square\right)$ is a compact, semi-topological semigroup, which we now denote by $w S$.

This space is denoted by $\mathcal{W} \mathcal{A P}(S)$ in [78].

Proposition 8.3. Let $S$ be a semigroup which is either weakly left cancellative or weakly right cancellative. Then $c_{0}(S) \subset W A P(S)$, and the map $\gamma_{W A P(S)}: S \rightarrow w S$ is injective.

Proof. Suppose that $S$ is a weakly left cancellative semigroup, say. It is sufficient to show that $\chi_{\{s\}} \in W A P(S)$ for each $s \in S$. For $s, t \in S$, we have $\chi_{\{s\}} \cdot t=\chi_{t^{-1}\{s\}}$. Since each set $t^{-1}\{s\}$ is finite, 
it follows that $L O\left(\chi_{\{s\}}\right) \subset c_{0}(S)$. Let $W$ be the weak-* closure of $L O\left(\chi_{\{s\}}\right)$ in $\ell^{\infty}(S)$. Then $W$ is weak-* compact. However, $W \subset c_{0}(S)$, and so the weak-* topology on $W$ coincides with the weak topology, and so $L O\left(\chi_{\{s\}}\right)$ is relatively weakly compact. Hence $\chi_{\{s\}} \in W A P(S)$.

The following characterization of $W A P(S)$ is given in [78, Theorem 21.18].

Proposition 8.4. Let $S$ be a semigroup, and let $\lambda \in S^{\mathbb{C}}$. Then $\lambda \in W A P(S)$ if and only if $\langle u \square v, \lambda\rangle=\langle u \diamond v, \lambda\rangle\left(u, v \in S^{*}\right)$.

The argument of the following result is essentially contained in [43].

THEOREM 8.5. Let $S$ be an infinite semigroup which is a subsemigroup of a group $G$, and let $X$ be an introverted $C^{*}$-subalgebra of $\ell^{\infty}(S)$ with $W A P(S) \subset X$. Then there is a non-empty subset $V$ of $\Phi_{X}$ such that

$$
s V t=V \quad(s, t \in G) \quad \text { and } \quad V \cap\left(\Phi_{X}^{*}\right)_{[2]}=\emptyset .
$$

Proof. Set

$$
U=S^{*} \backslash\left(\left(\overline{S^{*} \square S^{*}}\right) \cup\left(\overline{S^{*} \diamond S^{*}}\right)\right)
$$

an open subset of $S^{*}$. It follows from Proposition 6.31 that $U \neq \emptyset$.

Let $u \in U$ and $v \in S^{*}$ with $v \neq u$. There is a non-empty subset $F$ of $S$ such that $u \in F^{*} \subset U$ and $v \notin F^{*}$. Set $\lambda=\chi_{F}$. Then clearly

$$
\langle u \square v, \lambda\rangle=\langle u \diamond v, \lambda\rangle=0 \quad\left(u, v \in S^{*}\right),
$$

and so $\lambda \in W A P(S) \subset X$ by Proposition 8.4. Since $\langle u, \lambda\rangle \neq\langle v, \lambda\rangle$, we see that $\pi_{X}(u) \neq \pi_{X}(v)$.

Set $V=\pi(U)$, a non-empty subset $V$ of $\Phi_{X}$. Then $s V t=V$ for each $s, t \in G$. Assume towards a contradiction that $V \cap\left(\Phi_{X}^{*}\right)_{[2]} \neq \emptyset$. Then there exist elements $u \in U$ and $v_{1}, v_{2} \in S^{*}$ such that

$$
\pi(u)=\pi\left(v_{1}\right) \square \pi\left(v_{2}\right)=\pi\left(v_{1} \square v_{2}\right) .
$$

However $u \neq v_{1} \square v_{2}$, and so this contradicts the above remark.

The space $\left(\Phi_{A P(S)}, \square\right)$ is a compact, topological semigroup

which is sometimes denoted by $S_{a p}$ and sometimes by $b S$; it is denoted by $\mathcal{A P}(S)$ in [78]. We shall use the notation $b S$. This space is the Bohr compactification

of $S$.

We have the following maps. There are: continuous semigroup epimorphisms

$$
(\beta S, \square) \stackrel{\pi_{w}}{\longrightarrow}(w S, \square) \stackrel{\pi_{b}}{\longrightarrow}(b S, \square) ;
$$


isometric embeddings

$$
(C(b S), \cdot) \stackrel{T_{b}}{\longrightarrow}(C(w S), \cdot) \stackrel{T_{w}}{\longrightarrow}(C(\beta S), \cdot) ;
$$

continuous epimorphisms

$$
(M(\beta S), \square) \stackrel{T_{w}^{\prime}}{\longrightarrow}(M(w S), \square) \stackrel{T_{b}^{\prime}}{\longrightarrow}(M(b S), \square) .
$$

The maps $T_{w}^{\prime}$ and $T_{b}^{\prime} \circ T_{w}^{\prime}$ are of the form $\pi_{X}$ for $X=W A P(S)$ and $X=A P(S)$, respectively, where $\pi_{X}$ was defined in Chapter 2 .

Further, let us regard $\beta S$ as a subset of $\ell^{1}(\beta S) \subset M(\beta S), w S$ as a subset of $\ell^{1}(w S) \subset M(w G)$, and $b S$ as a subset of $\ell^{1}(b S) \subset$ $M(b S)$. Then we have $T_{w}^{\prime} \mid \beta S=\pi_{w}$ and $T_{b}^{\prime} \mid w S=\pi_{b}$, and so $T_{w}^{\prime}:\left(\ell^{1}(\beta S), \square\right) \rightarrow\left(\ell^{1}(w S), \square\right)$ and $T_{b}^{\prime}:\left(\ell^{1}(w S), \square\right) \rightarrow\left(\ell^{1}(b S), \square\right)$ are continuous epimorphisms.

Finally, we have continuous embeddings

$$
\Phi_{M(b S)} \longrightarrow \Phi_{M(w S)} \longrightarrow \Phi_{M(\beta S)} .
$$

Let $S$ be a semigroup, and set $A=\ell^{1}(S)$. We wish to clarify one point; the clarification involves $W A P(S)$. Set $M=A^{\prime \prime}=M(\beta S)$, and $L=\ell^{1}(\beta S)$. Then we have

$$
A \subset L \subset M \text { and } A^{\prime \prime} \subset L^{\prime \prime} \subset M^{\prime \prime} .
$$

Now take $\lambda \in A^{\prime}=C(\beta S) \subset \ell^{\infty}(\beta S)=L^{\prime}$ and $u \in \beta S$. Then we can calculate $\lambda \cdot u$ and $u \cdot \lambda$ in both $A^{\prime}$ and $L^{\prime}$. How are these functions related?

For $\lambda \in A^{\prime}$, the functions $\lambda \cdot u$ and $u \cdot \lambda$ in $A^{\prime}$ are defined in equation (2.12) as functions of $s \in S$, with $u=\delta_{u}$ for $\Phi \in A^{\prime \prime}$ and $s=\delta_{s}$ for $a \in A$. For $\lambda \in L^{\prime}$ and $u \in \beta S$, the functions $\lambda \cdot u$ and $u \cdot \lambda$ in $A^{\prime}$ are defined in equation (2.11) as functions on $\beta S$. Now define

$$
\left(\ell_{u} \lambda\right)(v)=\lambda(u \square v), \quad\left(r_{u} \lambda\right)(v)=\lambda(v \square u) \quad(v \in \beta S) .
$$

Then $u \cdot \lambda=r_{u} \lambda=\lambda \circ R_{u}$ and $\lambda \cdot u=\ell_{u} \lambda=\lambda \circ L_{u}$ as elements of $L^{\prime}$ (where $R_{u}$ and $L_{u}$ act on $(\beta S, \square)$ ), and we shall henceforth use the notations $\ell_{u} \lambda$ and $r_{u} \lambda$ for these functions. For the special case where $\lambda \in A^{\prime}$, the functions $u \cdot \lambda$ and $\lambda \cdot u$ in $A^{\prime}$ coincide with $\lambda \circ R_{u}$ and $\lambda \circ L_{u}$, respectively, as functions on $S$. Since $R_{u}$ is continuous on $\beta S$, we have $u \cdot \lambda=\lambda \circ R_{u}=r_{u} \lambda$ on $\beta S$, and so the two possible values of $u \cdot \lambda$ coincide. However, $L_{u}$ is not continuous on $\beta S$, and so the two possible values of $\lambda \cdot u$ may not coincide. Indeed, $\ell_{u} \lambda=\lambda \cdot u$ in $C(\beta S)$ for each $u \in \beta S$ if and only if $\lambda \in W A P(S)$.

Now let $\mu \in M$ and $u \in \beta S$. For $\lambda \in A^{\prime}$, we have

$$
\langle\mu \square u, \lambda\rangle=\langle\mu, u \cdot \lambda\rangle=\left\langle\mu, r_{u} \lambda\right\rangle .
$$


Let $\widetilde{\mu} \in L^{\prime \prime}$ be any extension of $\mu$ (so that $\widetilde{\mu} \mid A^{\prime}=\mu$ ). Then

$$
\langle\widetilde{\mu} \cdot u, \lambda\rangle=\langle\widetilde{\mu}, u \cdot \lambda\rangle=\langle\mu, u \cdot \lambda\rangle=\langle\mu \square u, \lambda\rangle \quad\left(\lambda \in A^{\prime}\right),
$$

and so

$$
(\widetilde{\mu} \cdot u) \mid A^{\prime}=\mu \square u
$$

We also have

$$
(s \cdot \widetilde{\mu}) \mid A^{\prime}=s \square \mu \quad(s \in S) .
$$

However there is no relation between $(u \cdot \widetilde{\mu}) \mid A^{\prime}$ and $u \square \mu$ for general $u \in \beta S$. To see this, let $S$ be a weakly concellative semigroup. We claim that, for each $u_{0} \in S^{*}$, there exists $\mu=\delta_{v_{0}} \in M$ such that $\mu$ has no extension $\widetilde{\mu} \in L^{\prime \prime}$ with $\left(u_{0} \cdot \widetilde{\mu}\right) \mid A^{\prime}=u_{0} \square \mu$. Indeed, by Theorem 12.20 , below (this is a well-known result), $u_{0} \notin \mathfrak{Z}_{t}^{(\ell)}(\beta S)$, and so there exists $v_{0} \in S^{*}$ with $u_{0} \square v_{0} \neq u_{0} \diamond v_{0}$; take $\lambda_{0} \in A^{\prime}$ with $\left\langle u_{0} \square v_{0}, \lambda_{0}\right\rangle=1$ and $\left\langle u_{0} \diamond v_{0}, \lambda_{0}\right\rangle=0$. Assume towards a contradiction that $\widetilde{\mu} \in L^{\prime \prime}$ is an extension of $\delta_{v_{0}} \in M$ with $\left(u_{0} \cdot \widetilde{\mu}\right) \mid A^{\prime}=u_{0} \square \mu$. Then

$$
\left\langle u_{0} \cdot \widetilde{\mu}, \lambda_{0}\right\rangle=\left\langle\widetilde{\mu}, \lambda_{0} \cdot \mu\right\rangle=\left\langle v_{0}, \lambda_{0} \cdot \mu\right\rangle
$$

because $\lambda_{0} \cdot \mu \in A^{\prime}$, and so $\left\langle u_{0} \cdot \widetilde{\mu}, \lambda_{0}\right\rangle=\left\langle u_{0} \diamond v_{0}, \lambda_{0}\right\rangle=0$. However $\left\langle u_{0} \square \mu, \lambda_{0}\right\rangle=1$. Thus $\left\langle u_{0} \cdot \widetilde{\mu}, \lambda_{0}\right\rangle \neq\left\langle u_{0} \square \mu, \lambda_{0}\right\rangle$, the required contradiction.

Submodules of $L^{\infty}(G)$ For the remainder of this chapter, $G$ is a locally compact group.

We shall again utilize the left and right translations $\ell_{t}$ and $r_{t}$, defined for functions $f$ on $G$ and $t \in G$ by the formulae:

$$
\left(\ell_{t} f\right)(s)=f(t s), \quad\left(r_{t} f\right)(s)=f(s t) \quad(s \in G) .
$$

The dual space of $L^{1}(G)$ is the space $\left(L^{\infty}(G),\|\cdot\|_{\infty}\right)$; this space is a $C^{*}$-algebra with respect to the pointwise product, which is defined save on locally null sets. We can also define the above left and right translations on $L^{\infty}(G)$.

The space $L^{1}(G)$ is a closed ideal in the Banach algebra $M(G)$, and so $L^{1}(G)$ is a Banach $M(G)$-bimodule. Hence its dual space $L^{\infty}(G)$ is also a Banach $M(G)$-bimodule; in particular, we can define $\delta_{s} \cdot \lambda$ and $\lambda \cdot \delta_{s}$ for $s \in G$ and $\lambda \in L^{\infty}(G)$. In fact, these elements of $L^{\infty}(G)$ are denoted by $s \cdot \lambda$ and $\lambda \cdot s$, respectively. We have

$$
\ell_{s} \lambda=\lambda \cdot s, \quad r_{s} \lambda=s \cdot \lambda \quad\left(t \in G, \lambda \in L^{\infty}(G)\right) .
$$

We shall also require the obvious definitions of $s \cdot \Lambda$ and $\Lambda \cdot s$ for $s \in G$ and $\Lambda \in L^{\infty}(G)^{\prime}$. Indeed,

$$
\langle s \cdot \Lambda, \lambda\rangle=\langle\Lambda, \lambda \cdot s\rangle, \quad\langle\Lambda \cdot s, \lambda\rangle=\langle\Lambda, s \cdot \lambda\rangle \quad\left(\lambda \in L^{\infty}(G)\right) .
$$


Definition 8.6. Let $G$ be a locally compact group. A closed subspace $X$ of $L^{\infty}(G)$ is a left-introverted $C^{*}$-subalgebra

if:

(i) $X$ is a $C^{*}$-subalgebra of $L^{\infty}(G)$;

(ii) $X$ is a left-introverted submodule of $L^{\infty}(G)$, where $L^{\infty}(G)$ is regarded as the dual module of $L^{1}(G)$.

Similarly, we define a right-introverted $C^{*}$-subalgebra of $L^{\infty}(G)$. The space $X$ is an introverted $C^{*}$-subalgebra if it is both a left- and a rightintroverted $C^{*}$-subalgebra.

DEFINITION 8.7. Let $G$ be a locally compact group. Then $L U C(G)$ and $R U C(G)$ denote the closed subspaces of $C B(G)$ consisting of the bounded, left (respectively, right) uniformly continuous functions on $G$, so that

$L U C(G)=\left\{\lambda \in C B(G): t \mapsto \ell_{t} \lambda, G \rightarrow C B(G)\right.$, is continuous $\}$; $R U C(G)=\left\{\lambda \in C B(G): t \mapsto r_{t} \lambda, G \rightarrow C B(G)\right.$, is continuous $\}$. We write $U C(G)$ for $L U C(G)$ in the case where $\operatorname{LUC}(G)=R U C(G)$.

Note that our notions of left and right uniformly continuous functions are interchanged from the definitions given in [72].

Let $\lambda \in L^{\infty}(G)$. It is shown in [21, Proposition 7.15] that $\lambda$ is in the equivalence class of a function in $L U C(G)$ (respectively, $R U C(G)$ ) if and only if the map $t \mapsto \lambda \cdot t, G \rightarrow L^{\infty}(G)$, (respectively, the map $t \mapsto t \cdot \lambda, G \rightarrow L^{\infty}(G)$, ) is continuous.

The spaces $L U C(G)$ and $R U C(G)$ are, respectively, left- and rightintroverted $C^{*}$-subalgebras of $L^{\infty}(G)$.

For $\lambda \in L^{\infty}(G)$, set $L O(\lambda)=\left\{\ell_{t} \lambda: t \in G\right\}$, essentially as before.

Definition 8.8. Let $G$ be a locally compact group. Then $\lambda$ is weakly almost periodic (respectively, almost periodic) if the set $L O(\lambda)$ is relatively compact in the weak (respectively, $\|\cdot\|-$ ) topology on $L^{\infty}(G)$.

The spaces of these functionals are denoted by $W A P(G)$ and $A P(G)$, respectively.

We have $A P(G)=A P\left(L^{1}(G)\right)$ and $W A P(G)=W A P\left(L^{1}(G)\right.$ ) (see [34, Theorem 1.1]). Further

$$
A P(G)=A P\left(G_{d}\right) \cap C B(G), \quad W A P(G)=W A P\left(G_{d}\right) \cap C B(G) .
$$

Each of the spaces $A P(G)$ and $W A P(G)$ is an introverted $C^{*}$-subalgebra of $L^{\infty}(G)$. Clearly

$$
A P(G) \subset W A P(G) \subset L U C(G) \subset C B(G) \subset L^{\infty}(G),
$$


and

$$
C_{0}(G) \subset W A P(G) .
$$

It was proved by Granirer [62] (see also [92, Corollary 4]) that we have $W A P(G)=L U C(G)$ if and only if $G$ is compact.

The character spaces of these algebras are denoted by

$$
\Phi_{A P(G)}, \quad \Phi_{W A P(G)}, \quad \Phi_{R U C(G)}, \quad \Phi_{L U C(G)}, \quad \Phi_{L^{\infty}(G)},
$$

respectively. (Elsewhere, these spaces are denoted by $\mathcal{W} \mathcal{A P}(S), G^{\mathcal{L C}}$, $G^{L U C}$, etc.; see $[\mathbf{1 0}],[\mathbf{4 3}]$, and [78].) We shall again use the notation $b G$ for $\Phi_{A P(G)}$, the Bohr compactification

of $G$, and $w G$ for $\Phi_{W A P(G)}$. Of course, we have identified $\Phi_{C B(G)}$ with $\beta G$. We have continuous surjections

$$
\Phi_{L^{\infty}(G)} \longrightarrow \Phi_{C B(G)} \longrightarrow \Phi_{L U C(G)} \longrightarrow \Phi_{W A P(G)} \longrightarrow \Phi_{A P(G)} .
$$

Since $C_{0}(G)$ separates the points of $G$, there is a natural embedding of $G$ in $\Phi_{W A P(G)}$, in $\Phi_{L U C(G)}$, and in $\Phi_{R U C(G)}$, and we regard $G$ as a dense, open subspace of these spaces. For a discussion of $A P(G)$ in the case where $G$ is a group, see [72, Chapter 18], for example. For certain groups $G$, the space $b G$ may be just a singleton, but $b G$ is an infinite group whenever $G$ is an infinite, maximally almost periodic group, and, in particular, whenever $G$ is abelian or a free group. More precisely, the map $\gamma$ is an injection if and only if $G$ is a maximally almost periodic group;

for more details on these topics, see [113, Chapter 3.2.16].

Let $G$ be a locally compact group, set $A=L^{1}(G)$, and let $X$ be a left-introverted $C^{*}$-subalgebra of $L^{\infty}(G)$ with $C_{0}(G) \subset X \subset L U C(G)$. Then $X$ is identified with the Banach algebra $C\left(\Phi_{X}\right)$, and $\left(X^{\prime}, \square\right)$ is identified with the Banach space $M\left(\Phi_{X}\right)$ of measures on $\Phi_{X}$, and so we can regard $\left(M\left(\Phi_{X}\right), \square\right)$ as a Banach algebra. As in Chapter $2, X^{\circ}$ is a closed ideal in $\left(L^{1}(G)^{\prime \prime}, \square\right)$ and there is a quotient map

$$
\pi_{X}:\left(L^{1}(G)^{\prime \prime}, \square\right) \rightarrow\left(L^{1}(G)^{\prime \prime}, \square\right) / X^{\circ}=\left(X^{\prime}, \square\right) .
$$

The space $X$ is also a left-introverted subspace of $\ell^{\infty}(G)$, and so $X^{\prime}$ is also a quotient of $\left(\ell^{1}(G)^{\prime \prime}, \square\right)$; fortunately, this new product coincides with $\square[\mathbf{2 1}$, Theorem 5.15]. Similarly, suppose that $X$ is a leftintroverted $C^{*}$-subalgebra of $\ell^{\infty}(G)$ such that $X \subset C B(G) \subset L^{\infty}(G)$. Then $X$ is a left-introverted $C^{*}$-subalgebra of $L^{\infty}(G)$; again the two possible definitions of $\square$ agree, and in fact $X \subset L U C(G)[\mathbf{9 0}$, Lemma 3], [107].

Let $X$ be an introverted $C^{*}$-subalgebra of $L^{\infty}(G)$ with $C_{0}(G) \subset X$, and set $E=C_{0}(G)$. The quotient map

$$
R_{X}: \Phi \mapsto \Phi \mid E, \quad\left(X^{\prime}, \square\right) \rightarrow\left(E^{\prime}, \square\right)=(M(G), \star),
$$


is a continuous epimorphism. Further, the map

$$
q_{X, G}:=q_{G} \circ R_{X}:\left(X^{\prime}, \square\right) \rightarrow\left(\ell^{1}(G), \star\right),
$$

is a continuous epimorphism. (Recall that $q_{G}$ was defined in equation $(1.5)$.)

Proposition 8.9. Let $G$ be a locally compact group, and let $X$ be an introverted $C^{*}$-subalgebra of $L^{\infty}(G)$ with $C_{0}(G) \subset X$. Suppose that there is a non-zero, continuous point derivation at the discrete augmentation character on $M(G)$. Then there is a non-zero, continuous point derivation at the discrete augmentation character on $X^{\prime}$, and so $X^{\prime}$ is not weakly amenable.

Proof. This follows from Proposition 2.2(ix).

Let $G$ be a locally compact group, and let $X$ be a left-introverted subspace of $\ell^{\infty}(G)$ with $C_{0}(G) \subset X \subset C B(G)$. For $\mu \in M(G)$, define $\theta \mu \in X^{\prime}$ by

$$
\langle\theta \mu, \lambda\rangle=\int_{G} \lambda(s) \mathrm{d} \mu(s) \quad(\lambda \in X) .
$$

Clearly $\theta \mu \mid C_{0}(G)=\mu(\mu \in M(G))$. In particular, $\left\langle\theta \delta_{s}, \lambda\right\rangle=\lambda(s)$ for each $s \in G$. The map $\theta: M(G) \rightarrow X^{\prime}$ is a linear isometry. (For a discussion of an embedding of $(M(G), \star)$ into $\left(A^{\prime \prime}, \square\right)$ which is a right inverse to this map $R_{A^{\prime}}$, see [53, Chapter 2] and [21].) We define

$$
\widetilde{\varphi}_{X, G}=\varphi_{G} \circ q_{X, G} .
$$

Then again $\widetilde{\varphi}_{X, G}$ is a character on $\left(X^{\prime}, \square\right)$, called the discrete augmentation character. We also regard the map $\varphi_{X, G} \circ q_{G}$ as the discrete augmentation character

on $M(G)$; this coincides with the definition in [23].

Proposition 8.10. Let $G$ be a locally compact group, and let $X$ be a left-introverted $C^{*}$-subalgebra of $\ell^{\infty}(G)$ with $C_{0}(G) \subset X \subset C B(G)$. Then $\theta:(M(G), \star) \rightarrow\left(M\left(\Phi_{X}\right), \square\right)$ is an isometric embedding, and

$$
M\left(\Phi_{X}\right)=\theta(M(G)) \ltimes C_{0}(G)^{\circ},
$$

where $C_{0}(G)^{\circ}$ is the annihilator of $C_{0}(G)$ in $X^{\prime}$.

Proof. This is [96, Lemma 4.1].

In fact, in the above case, we regard $M(G)$ as a closed subalgebra of $M\left(\Phi_{X}\right)$. We also regard $\Phi_{X}$ as a compact subset of $M\left(\Phi_{X}\right)$; it is again easily checked that $\left(\Phi_{X}, \square\right)$ is a compact, right topological semigroup. 
(The character space $\Phi_{L^{\infty}(G)}$ is discussed in [101]; it is a very complicated object, and it is not a semigroup unless $G$ is discrete or compact. See also [24] for a long discussion of $\Phi_{L^{\infty}(G)}$.)

Since the space $(b G, \square)$ is a compact topological group, we can define the measure algebra $(M(b G), \star)$ on $b G$; it is immediate from the definitions that the product $\star$ coincides with the product $\square$ on $M(b G)$, and so we have a continuous epimorphism

$$
\pi:(M(\beta G), \square) \rightarrow(M(b G), \star) .
$$

Clearly $\pi:\left(M\left(G^{*}\right), \square\right) \rightarrow(M(b G \backslash G), \star)$ is also a continuous epimorphism.

Again, let $X$ be a left-introverted $C^{*}$-subalgebra of $\ell^{\infty}(G)$ with $C_{0}(G) \subset X \subset C B(G)$. Let $u, v \in \Phi_{X}$, and take nets $\left(s_{\alpha}\right)$ and $\left(t_{\beta}\right)$ in $G$ with $\lim _{\alpha} s_{\alpha}=u$ and $\lim _{\beta} t_{\beta}=v$. Again we have

$$
u \square v=\lim _{\alpha} \lim _{\beta} s_{\alpha} t_{\beta} .
$$

Let $S$ be a subset of $G$. Then $\bar{S}$ denotes the closure of $S$ in $\Phi_{X}$, and $S^{*}=\bar{S} \backslash S$. In particular, $\Phi_{X}^{*}=\Phi_{X} \backslash G$, so that $\Phi_{X}^{*}$ is the growth of $G$ in $\Phi_{X}$.

Let $\mu \in M\left(\Phi_{X}\right)$. Then, as before, we define $L_{\mu}, R_{\mu} \in \mathcal{B}\left(M\left(\Phi_{X}\right)\right)$ as the map

$$
L_{\mu}: \nu \mapsto \mu \square \nu, \quad R_{\mu}: \mu \mapsto \nu \square \mu, \quad M\left(\Phi_{X}\right) \rightarrow M\left(\Phi_{X}\right) .
$$

We shall often write $u v$ for $u \square v$ when $u, v \in \Phi_{X}$.

Proposition 8.11. Let $G$ be a locally compact group.

(i) Let $K$ and $L$ be disjoint, non-empty, compact subsets of $\Phi_{L U C(G)}$. Then there is a symmetric neighbourhood $W$ of $e_{G}$ with $K \cap W L=\emptyset$.

(ii) Let $A$ and $B$ be subsets of $G$ such that $\bar{A} \cap \bar{B} \neq \emptyset$ in $\Phi_{L U C(G)}$. Then $A \cap W B \neq \emptyset$ for each neighbourhood $W$ of $e_{G}$.

(iii) Let $A$ be a subset of $G$. Then $W \bar{A}$ is a neighbourhood of $\bar{A}$ in $\Phi_{X}$ for each neighbourhood $W$ of $e_{G}$.

Proof. (i) Choose $\lambda \in C\left(\Phi_{L U C(G)}\right)$ with $\lambda\left(\Phi_{L U C(G)}\right) \subset \mathbb{I}$, with $\lambda \mid K=1$, and with $\lambda \mid L=0$. Since $\lambda \mid G \in L U C(G)$, there is a symmetric neighbourhood $W$ of $e_{G}$ in $G$ such that

$$
|\lambda(w s)-\lambda(s)|<\frac{1}{2} \quad(s \in G, w \in W) .
$$

For each $w \in W$ and $u \in \Phi_{L U C(G)}$, we have $|\lambda(w u)-\lambda(u)| \leq 1 / 2$, and so $|\lambda(w u)| \leq 1 / 2(w \in W, u \in L)$. Hence $K \cap W L=\emptyset$.

(ii) and (ii) These are immediate from [78, Exercise 21.5.3]. 
Let $G$ be a locally compact group, and let $X$ be a left-invariant subspace of $L^{\infty}(G)$. We recall from Definition 2.31 that the topological centre

of $X^{\prime}$ is

$$
\mathfrak{Z}_{t}\left(X^{\prime}\right)=\left\{\mu \in M\left(\Phi_{X}\right): L_{\mu} \text { is weak-*-continuous on } X^{\prime}\right\} .
$$

In the case where $X$ is introverted, we see easily that

$$
\mathfrak{Z}_{t}\left(X^{\prime}\right)=\left\{\mu \in M\left(\Phi_{X}\right): \mu \square \nu=\mu \diamond \nu\left(\nu \in M\left(\Phi_{X}\right)\right)\right\} .
$$

Thus $\mathfrak{Z}_{t}\left(X^{\prime}\right)$ coincides with our previous definition of $\mathfrak{Z}_{t}^{(\ell)}(M(\beta G))$ in the case where $X=L^{\infty}(G)$.

We recall some history of calculations involving the group algebra $L^{1}(G)$. First, $L^{1}(G)$ is Arens regular if and only if $G$ is finite: this was first proved by Civin and Yood [16] for $G$ abelian and in the general case by Young [139]. In fact, $L^{1}(G)$ is always strongly Arens irregular. This was proved for $G$ compact by Işik, Pym, and Ülger in [82]. The general case was first proved by Lau and Losert in [95]; see also [99]. A different proof in the abelian case is given by Ghahramani and McClure in [52], and a proof of a generalization of this theorem is given by Neufang in $[\mathbf{1 1 0}]$.

TheOREM 8.12. Let $G$ be a locally compact group. Then

$$
\mathfrak{Z}_{t}\left(L U C(G)^{\prime}\right)=M(G) .
$$

Proof. This was first proved in [92]; see also [21, Theorem 11.9] and $[\mathbf{1 1 0}]$ for a more general result.

We shall extend the above result considerably in Theorem 12.24.

For an extension of the above result to topological groups which are not necessarily locally compact, see [41].

As a comparison, we state the following result [21, Theorem 11.4].

THEOREM 8.13. Let $G$ be a locally compact group, and let $X$ be a $\|\cdot\|$-closed $L^{1}(G)$-submodule of $L^{\infty}(G)$ such that $X \subset W A P(G)$. Then $X$ is introverted

$$
\text { and } \mathfrak{Z}_{t}\left(X^{\prime}\right)=X^{\prime}
$$

For each $u \in \Phi_{X}$, we have the map $L_{u} \mid \Phi_{X}: v \mapsto u \square v, \Phi_{X} \rightarrow \Phi_{X}$.

Definition 8.14. Let $G$ be a locally compact group, and let $X$ be a left-introverted subspace of $L^{\infty}(G)$. Then

$$
\mathfrak{Z}_{t}\left(\Phi_{X}\right)=\left\{u \in \Phi_{X}: L_{u} \mid \Phi_{X} \text { is continuous }\right\} .
$$


Clearly $G \subset \mathfrak{Z}_{t}\left(\Phi_{X}\right)$ and $\mathfrak{Z}_{t}\left(X^{\prime}\right) \cap \Phi_{X} \subset \mathfrak{Z}_{t}\left(\Phi_{X}\right)$. Our definition of $\mathfrak{Z}_{t}\left(\Phi_{X}\right)$ coincides with that of $\Lambda\left(\Phi_{X}\right)$ given in [78, Definition 2.4]. In the case where $X$ is an introverted subspace of $L^{\infty}(G)$, we have

$$
\mathfrak{Z}_{t}\left(\Phi_{X}\right)=\left\{u \in \Phi_{X}: u \square v=u \diamond v\left(v \in \Phi_{X}\right)\right\},
$$

and so our new definition coincides with that of $\mathfrak{Z}_{t}^{(\ell)}(\beta G)$ given in Definition 6.11 in the case where $G$ is discrete and $X=\ell^{\infty}(G)$.

TheOREM 8.15. Let $G$ be a locally compact group. Then

$$
\mathfrak{Z}_{t}\left(\Phi_{L U C(G)}\right)=G \text {. }
$$

Proof. This is proved in [98]; for later proofs of slightly stronger results, see [118] and [45, Theorem 4.2]. 



\section{CHAPTER 9}

\section{Amenability for semigroups}

In this chapter, we shall discuss left- and right-amenability for semigroups. Our main results will give new information about the support in $\beta S$ of left-invariant measures on a semigroup $S$.

The seminal paper on the amenability of semigroups is $[29]$; the present classic reference for the theory of amenable groups and semigroups is $[\mathbf{1 1 6}]$.

Definitions As usual, we begin with basic definitions and properties, now of invariant means and amenability for semigroups.

Definition 9.1. Let $S$ be a semigroup, and let $\mu \in M(\beta S)$. Then $\mu$ is a mean on $S$ if

$$
\|\mu\|=\langle\mu, 1\rangle=1,
$$

left-invariant if $s \cdot \mu=\mu(s \in S)$,

right-invariant if $\mu \cdot s=\mu(s \in S)$, and invariant if it is both left-invariant and right-invariant.

$A$ subset $X$ of $M(\beta S)$ is $S$-invariant

if $S \cdot X \cdot S \subset X$ and $\beta S$-invariant

if $\beta S \square X \square \beta S \subset X$.

The semigroup $S$ is left-amenable (respectively, right-amenable, amenable)

if there is a left-invariant mean (respectively, right-invariant mean, invariant mean) on $S$.

The sets of means and of left-invariant means on $S$ are denoted by $\mathfrak{M}(S)$ and $\mathfrak{L}(S)$, respectively.

We shall also require the following standard definition, which is closely related to Definition 9.1.

DEFINITION 9.2. Let $G$ be a locally compact group, and consider an element $\Lambda \in L^{\infty}(G)^{\prime}$. Then $\Lambda$ is a mean on $G$ if

$$
\|\Lambda\|=\langle\Lambda, 1\rangle=1
$$

left-invariant if $s \cdot \Lambda=\Lambda(s \in G)$, 
and invariant if

$$
s \cdot \Lambda=\Lambda \cdot s=\Lambda \quad(s \in G) .
$$

The group $G$ is amenable

if there is a left-invariant mean on $G$.

There is also some theory of amenability for more general topological semigroups [93], [109].

Let $S$ be a semigroup. It is clear that $\mathfrak{M}(S)$ and $\mathfrak{L}(S)$ are $\beta S$ invariant, weak-* compact, convex subsets of $M(\beta S)$. Clearly $\mathfrak{M}(S)$ is a subsemigroup of $(M(\beta S), \square)$, and so $(\mathfrak{M}(S), \square)$ is a compact, right topological semigroup. Thus this semigroup has a minimum ideal $K(\mathfrak{M}(S))$, and it contains minimal idempotents; for this, see Example 3.44 .

A semigroup which is both left-amenable and right-amenable is amenable; a left-amenable

group is amenable [19, Proposition 3.3.49]; a subgroup of an amenable group is amenable, and a left or right ideal in a left-amenable semigroup is itself left-amenable, and so a subgroup of the form $s S t$ of an amenable semigroup $S$, where $s, t \in S$, is amenable; see [116, Chapter 1] for details of these results. However a subsemigroup of an amenable group need not be itself left-amenable [80]. Each abelian semigroup is amenable; the standard example of a non-amenable group is $\mathbb{F}_{2}$.

It is interesting to note that a left-amenable, cancellative semigroup is always a subsemigroup of an amenable group; this is a result of Wilde and Witz [133], given as [116, (1.27)].

The cardinality $|\mathfrak{L}(S)|$ of the set of left-invariant means on a semigroup $S$ has been much studied; see [116, Chapter 7] for a definitive account. The general conclusion is, as Paterson remarks, 'remarkably simple': the cardinality is the 'largest possible' unless the semigroup possesses some strong property which limits this cardinality. For example, it is proved in [116, Corollary (7.8)] that

$$
|\mathfrak{L}(G)|=2^{2^{|G|}}
$$

for an infinite, amenable group $G$. The value of $|\mathfrak{L}(S)|$ for semigroups $S$ is also discussed in [116, Theorem (7.26)] (see also [133]); often $|\mathfrak{L}(S)|$ is a large infinite number. However, we are more interested in special cases where $|\mathfrak{L}(S)|$ is finite. There are semigroups $S$ with $|\mathfrak{L}(S)|=1$. Indeed, let $T$ be any semigroup, and let $S=T^{\circ}$. Clearly in this case $|\mathfrak{L}(S)|=1$ : the unique (left-)invariant mean in $M(\beta S)$ is $\delta_{o}$.

Proposition 9.3. Let $S$ be a left-amenable semigroup, and suppose that $|\mathfrak{L}(S)|=1$. Then $S$ contains exactly one finite group ideal. 
Proof. This is explicitly stated in [87, Corollary 4.6]; it also follows from the solution given to Problem (7-14) in [116].

Proposition 9.4. Let $S$ be a semigroup, and let $\mu \in M(\beta S)$ be left-invariant. Then

$$
\nu \square \mu=\nu(\beta S) \mu=\langle\nu, 1\rangle \mu=\left(\int_{\beta S} \mathrm{~d} \nu\right) \mu \quad(\nu \in M(\beta S)) .
$$

In particular, $u \square \mu=\mu(u \in \beta S)$ and $\mu \square \mu=\langle\mu, 1\rangle \mu$. Further,

$$
\mu\left(s^{-1} U\right)=\mu(U) \quad(s \in S)
$$

for each Borel subset $U$ of $\beta S$. Each left-invariant mean is a minimal idempotent in the algebra $(M(\beta S), \square)$

and in the semigroup $(\mathfrak{M}(S), \square)$.

Proof. Equation (9.1) holds for $\nu=s \in S$, and hence for each $\nu \in \ell^{1}(S)$ with finite support; it now holds for each $\nu \in M(\beta S)$ by taking weak- $*$ limits.

For each $s \in S$ and $F \subset S$, we have

$$
\begin{aligned}
\mu(\bar{F}) & =\left\langle\mu, \chi_{F}\right\rangle=\left\langle s \cdot \mu, \chi_{F}\right\rangle=\left\langle\mu, \chi_{F} \cdot s\right\rangle \\
& =\left\langle\mu, \chi_{s^{-1} F}\right\rangle=\mu\left(\overline{s^{-1} F}\right)=\mu\left(s^{-1} \bar{F}\right),
\end{aligned}
$$

whence (9.2) holds for $U=\bar{F}$. The general case follows by taking intersections and unions of sets and noting that $\mu$ is a regular measure.

Let $\mu \in \mathfrak{L}(S)$. By (9.1), we have $\mu \in \mathfrak{I}(M(\beta S)) \backslash\{0\}$. Now take $\nu \in \mathfrak{I}(M(\beta S)) \backslash\{0\}$ with $\nu \leq \mu$. Then $\nu \square \mu=\nu$. But $\nu \square \mu=\langle\nu, 1\rangle \mu$ by (9.1), and so $\nu=\alpha \mu$ for $\alpha=\langle\nu, 1\rangle$. Since $\nu \neq 0$, necessarily $\alpha \neq 0$. Since $\nu \square \nu=\nu$, we have $\alpha^{2}=\alpha$, and so $\alpha=1$. Thus $\nu=\mu$, and hence $\mu$ is a minimal idempotent in the algebra $M(\beta S)$. Suppose that $\nu \in \mathfrak{M}(S)$ and that $\nu \leq \mu$, so that $\nu \square \mu=\nu$. Again $\nu \square \mu=\mu$, and so $\nu=\mu$.

Proposition 9.5. Let $S$ be a semigroup such that the semigroup $(\beta S, \square)$ is amenable. Then $S$ is amenable.

Proof. Let $\mu \in \ell^{\infty}(\beta S)^{\prime}=M\left(\beta\left((\beta S)_{d}\right)\right)$ be an invariant mean on $\beta S$. Then $C(\beta S)$ is an $S$-invariant subspace of $\ell^{\infty}\left((\beta S)_{d}\right)$, and so $\mu \mid C(\beta S)$ belongs to $M(\beta S)$ and is an invariant mean on $S$.

Definition 9.6. Let $S$ be a semigroup. Set

$$
\begin{aligned}
& M_{0}(\beta S)=\{\mu \in M(\beta S):\langle\mu, 1\rangle=0\}, \\
& M_{1}(\beta S)=\{\mu \in M(\beta S):\langle\mu, 1\rangle=1\} .
\end{aligned}
$$


Clearly $\left(M_{0}(\beta S), \square\right)$ and $\left(M_{1}(\beta S), \square\right)$ are both subsemigroups of the semigroup $(M(\beta S), \square)$, and so both are right topological semigroups. Now take $\mu \in \mathfrak{I}(M(\beta S))$, and set $\alpha=\langle\mu, 1\rangle$. Then $\alpha^{2}=\alpha$, and so $\alpha=0$ or $\alpha=1$. Thus

$$
\mathfrak{I}(M(\beta S)) \subset M_{0}(\beta S) \cup M_{1}(\beta S) .
$$

The semigroup $(M(\beta S), \square)$ has a minimum ideal, namely $\{0\}$. However, we are interested in the minimum ideal $K\left(M_{1}(\beta S)\right)$.

Proposition 9.7. Let $S$ be a left-amenable semigroup. Then:

(i) the minimum ideal $K\left(M_{1}(\beta S), \square\right)$ exists, and consists of the left-invariant elements of $M_{1}(\beta S)$.

(ii) $\mathfrak{L}(S)=K(\mathfrak{M}(S), \square)$.

Proof. (i) Take $\mu \in M_{1}(\beta S)$ with $\mu$ left-invariant. By Proposition $9.4, \mu$ is an idempotent and $M_{1}(\beta S) \square \mu=\{\mu\}$, and so $\{\mu\}$ is a minimal left ideal in $M_{1}(\beta S)$. By Theorem 3.7(iii), the smallest ideal $K\left(M_{1}(\beta S)\right)$ exists; every minimal left ideal is a singleton of the above form, and so $K\left(M_{1}(\beta S)\right)$ is the set of left-invariant elements in $M_{1}(\beta S)$.

(ii) $\left[\mathbf{1 3 5}\right.$, Theorem 2.11] Let $\mu \in \mathfrak{L}(S) \subset M_{1}(\beta S)$. Then $\mu$ is an idempotent in $\mathfrak{M}(S)$, and $\{\mu\}$ is a minimal left ideal in $\mathfrak{M}(S)$. By Proposition 3.8(i), $\mathfrak{L}(S)=K(\mathfrak{M}(S))$.

Let $S$ be a semigroup. It would be interesting to characterize $K(\mathfrak{M}(S), \square)$ in the case where $S$ is not left-amenable. For example, is this ideal always weak-* closed? Temporarily set $K_{1}=K(\mathfrak{M}(S), \square)$ and $K_{2}=K(\mathfrak{M}(S), \diamond)$. Then clearly $K_{1}=K_{2}$ when $S$ is abelian, and $K_{1} \cap K_{2} \neq \emptyset$ when $S$ is amenable; always $\overline{K_{1}} \cap \overline{K_{2}} \neq \emptyset$.

Proposition 9.8. (i) Let $\mu$ be a right-invariant mean on a semigroup $S$, and let $L$ be a left ideal of $S$. Then $\left\langle\mu, \chi_{L}\right\rangle=1$.

(ii) Let $S$ be a right-amenable semigroup. Then $S$ has at most one minimal left ideal.

(iii) Let $S$ be a left-amenable semigroup. Then $S$ has at most one minimal right ideal.

Proof. (i) Let $a \in L$. Then $L a^{-1}=S$, and so $a \cdot \chi_{L}=\chi_{S}$ by (4.3). Hence $\left\langle\mu, \chi_{L}\right\rangle=\left\langle\mu \cdot a, \chi_{L}\right\rangle=\left\langle\mu, a \cdot \chi_{L}\right\rangle=\left\langle\mu, \chi_{S}\right\rangle=1$.

(ii) Let $\mu$ be a right-invariant mean on $S$. Assume that $L_{1}$ and $L_{2}$ are two distinct minimal ideals in $S$. Then $L_{1} \cap L_{2}=\emptyset$, and so $\chi_{L_{1} \cup L_{2}}=\chi_{L_{1}}+\chi_{L_{2}}$. By (i), $\mu\left(L_{1} \cup L_{2}\right)=2$, a contradiction of the fact that $\|\mu\|=1$.

(iii) This follows by applying (ii) to $S^{\text {op }}$. 
The following result is stated without proof in [33, page 145].

Proposition 9.9. Let $S$ be a simple, regular, amenable semigroup such that $E(S)$ is finite. Then $S$ is a group.

Proof. Since $E(S)$ is finite, there exists a minimal idempotent, say $p$, in $S$. Since $S$ is regular, it follows from Proposition 3.6(i) that $S p$ is a minimal left ideal in $S$, and $p \in S p$. Thus the structure theorem 3.7 applies. Since $S$ is simple, $K(S)=S$.

By Theorem 3.7(iii), $K(S)$ is the union of the minimal left ideals of $S$. Since $S$ is amenable, it follows from Lemma 9.8(ii) that $S$ has at most one minimal left ideal, and so $S$ is a minimal left ideal in $S$. Similarly, $S$ is a minimal right ideal in $S$. By the structure theorem $3.7(\mathrm{iv}), S=S_{[2]}$ is a group.

THEOREM 9.10. Let $S$ be an infinite semigroup.

(i) Suppose that $S$ is weakly left cancellative.

Then $\beta S$ is not right-amenable.

(ii) Suppose that $S$ is cancellative. Then $\beta S$ is not left-amenable.

Proof. (i) By Proposition 6.23(i), ( $\beta S, \square)$ contains at least two distinct minimal left ideals, and so this follows from Proposition 9.8(ii).

(ii) By Proposition 6.23(ii), $(\beta S, \square)$ contains at least two distinct minimal right ideals, and so this follows from Proposition 9.8(iii).

We remark on the limits of the above theorem. A semigroup $S$ is said to be extremely left-amenable

if there exists $u \in \beta S$ such that $s \square u=u(s \in S)$. In this case, we have $v \square u=u(v \in \beta S)$; define $\mathrm{M} \in\left(\ell^{\infty}(\beta S)\right)^{\prime}$ by setting

$$
\langle\mathrm{M}, \Lambda\rangle=\Lambda(u) \quad\left(\Lambda \in \ell^{\infty}(\beta S)\right) .
$$

Then $\mathrm{M}$ is a left-invariant mean on $(\beta S, \square)$, and so $(\beta S, \square)$ is leftamenable in this case. A more general concept that implies the leftamenability of $(\beta S, \square)$ is given in [89].

The class of extremely left-amenable semigroups can be characterized algebraically. Indeed, a semigroup $S$ is extremely left-amenable if and only if, for each $a, b \in S$, there exists $c \in S$ with

$$
a c=b c=c .
$$

That each extremely left-amenable semigroup has this property is due to Granirer [61], and the converse is due to Mitchell [106]. There are many examples of infinite semigroups which are extremely leftamenable. For example, the semigroup $\mathbb{N}_{\vee}$ of Example 3.36 is weakly 
cancellative and extremely left-amenable. Let $S$ be any infinite semigroup with a zero. Then $S$ and $(\beta S, \square)$ are extremely amenable semigroups (in the obvious sense), and so neither clause (i) nor clause (ii) of Theorem 9.10 holds for an arbitrary infinite semigroup.

The support of means Let $S$ be a semigroup. We wish to determine some properties of the supports of means and of left-invariant means on $S$. For example, let $\mu \in \mathfrak{M}(S)$. Then [133, Lemma 4.1]

$$
\operatorname{supp} \mu=\bigcap\left\{\bar{F}: F \subset S,\left\langle\mu, \chi_{F}\right\rangle=1\right\} \text {. }
$$

Definition 9.11. Let $S$ be a semigroup. Then

$$
L(\beta S)=\bigcup\{\operatorname{supp} \mu: \mu \in \mathfrak{L}(S)\},
$$

where we take $L(\beta S)=\emptyset$ in the case where $S$ is not left-amenable.

Let $\left(\mu_{n}\right)$ be a sequence in $\mathfrak{L}(S)$, and set $\mu=\sum_{n=1}^{\infty} \mu_{n} / 2^{n}$. Then $\mu \in \mathfrak{L}(S)$ and $\operatorname{supp} \mu=\overline{\bigcup\left\{\operatorname{supp} \mu_{n}: n \in \mathbb{N}\right\}}$.

We enquire when the set $L(\beta S)$ is closed in $\beta S$. In fact, we do not even know whether or not $L(\beta \mathbb{N})$ is closed in $\beta \mathbb{N}$.

Proposition 9.12. Let $S$ be an infinite, cancellative semigroup, and let $\mu \in \mathfrak{L}(S)$. Then $\mu \in M_{c}\left(S^{*}\right)$, and so $\overline{L(\beta S)} \subset S^{*}$.

Proof. Let $v \in \beta S$. By Proposition $6.5, s^{-1} v$ is a singleton for each $s \in S$ and all these points are distinct. Let $s \in S$. Since $s \cdot \mu=\mu$, we have $\mu\left(\left\{s^{-1} v\right\}\right)=\mu(\{v\})$, and so $\mu(\{v\})=0$. Thus $\mu \in M_{c}\left(S^{*}\right)$.

The following remark was made in [40, Proposition 2.1], following a partial result in $[\mathbf{1 3 3}$, Theorem 4.3].

Proposition 9.13. Let $S$ be a left-amenable semigroup, and take $\mu \in \mathfrak{L}(S)$. Then supp $\mu$ is a closed, left ideal in $\beta S$.

Proof. Let $\mu \in \mathfrak{L}(S)$, and take $s \in S$ and $F \subset S$ such that $\left\langle\mu, \chi_{F}\right\rangle=1$. Then $\left\langle\mu, \chi_{s^{-1} \cdot F}\right\rangle=1$, and so $\operatorname{supp} \mu \subset s^{-1} \cdot \bar{F}$. Hence $s \cdot \operatorname{supp} \mu \subset \bar{F}$. It follows that $s \cdot \operatorname{supp} \mu \subset \operatorname{supp} \mu$. Since rightmultiplication in $\beta S$ is continuous, $u \square \operatorname{supp} \mu \subset \operatorname{supp} \mu(u \in \beta S)$. Thus supp $\mu$ is a left ideal in $\beta S$.

EXAMPLE 9.14. (i) Let $S$ be a right zero semigroup, so that

$$
u \square v=v \quad(u, v \in \beta S) ;
$$


$S$ is both left amenable and left cancellative. For each $v \in \beta S$, we have $\delta_{v} \in \mathfrak{L}(S)$, and so $v \in L(\beta S)$. Thus we cannot replace 'cancellative' by 'left cancellative' in Proposition 9.12. In fact, $K(\beta S)=L(\beta S)=\beta S$.

(ii) Let $S=\mathbb{N}_{\vee}$. Then $\mathfrak{L}(S)=\left\{\mu \in M\left(S^{*}\right):\|\mu\|=\langle\mu, 1\rangle=1\right\}$, and so $L(\beta S)=S^{*}$, which is closed in $\beta S$. In particular, $\delta_{v} \in \mathfrak{L}(S)$ for $v \in S^{*}$, and so we cannot replace 'cancellative' by 'weakly cancellative' in Proposition 9.12.

Proposition 9.15. Let $S$ be a semigroup, and let $L$ be a minimal left ideal in $\beta S$. Then there is a minimal idempotent $\mu_{0} \in \mathfrak{M}(S)$ with $\operatorname{supp} \mu_{0} \subset L$.

Proof. Choose $u \in L$. Then $s \cdot u \in \mathfrak{M}(S) \cap L(s \in S)$, and so $\operatorname{supp}(\mu \square u) \subset L(\mu \in \mathfrak{M}(S))$. Since $\{\mu \square u: \mu \in \mathfrak{M}(S)\}$ is a left ideal in $(\mathfrak{M}(S), \square)$, it contains a minimal idempotent, say $\mu_{0}$. The element $\mu_{0}$ has the required properties.

Parts of the next result were given in [97, Lemma 4] by a different argument.

Proposition 9.16. Let $S$ be a left-amenable semigroup.

(i) Let I be a minimal left ideal of $(\beta S, \square)$. Then there is a leftinvariant mean $\mu_{0}$ on $S$ such that $\operatorname{supp} \mu_{0}=I$.

(ii) $L(\beta S)$ and $\overline{L(\beta S)}$ are ideals in $(\beta S, \square)$.

(iii) $K(\beta S) \subset L(\beta S)$ and $\overline{K(\beta S)} \subset \overline{L(\beta S)}$.

Proof. (i) Let $\mu \in \mathfrak{L}(S)$, and take $v \in \beta S$. Then $\mu \square v \in \mathfrak{L}(S)$, and we have $\operatorname{supp}(\mu \square v)=(\operatorname{supp} \mu) \square v$ by (7.3). Choose $v \in I$, and set $\mu_{0}=\mu \square v$. Then $\operatorname{supp} \mu_{0} \subset I$. For $s \in S, s \cdot \operatorname{supp} \mu_{0}=\operatorname{supp} \mu_{0}$, and so supp $\mu_{0}$ is a closed left ideal in $\beta S$. Hence supp $\mu_{0}=I$.

(ii) It is immediate from Proposition 9.13 that $L(\beta S)$ is a left ideal. As in (i), $L(\beta S)$ is a right ideal. By Proposition $6.14(\mathrm{ii}), \overline{L(\beta S)}$ is an ideal in $\beta S$.

(iii) This is now immediate.

Let $S$ be an infinite, weakly cancellative semigroup. We remark that there is no measure $\mu \in M(\beta S)$ such that supp $\mu \supset \overline{K(\beta S)}$. For set $K=K(\beta S)$, and take $\mu \in M(\beta S)$. It follows from [15, Theorem 2.3(i)] that there is a family $\left\{U_{\alpha}: \alpha<\omega_{1}\right\}$ of open subsets of $S^{*}$ such that $U_{\alpha} \cap K \neq \emptyset\left(\alpha<\omega_{1}\right)$ and $U_{\alpha} \cap U_{\beta} \cap K=\emptyset\left(\alpha, \beta<\omega_{1}, \alpha \neq \beta\right)$. Of course $|\mu|\left(U_{\alpha}\right)=0$ for all save countably many values of $\alpha$, and so there exists $\alpha<\omega_{1}$ such that supp $\mu \subset \bar{K} \backslash U_{\alpha} \subsetneq \bar{K}$. 
Recall that each cancellative, abelian semigroup is maximally almost periodic.

Proposition 9.17. Let $S$ be an infinite, maximally almost periodic semigroup, and let $\mu \in \mathfrak{L}(S)$. Then

$$
\mu(\overline{E(\beta S)})=0 .
$$

Proof. Set $F=\overline{E(\beta S)}$ and $\alpha=\mu(F)$.

Let $h: S \rightarrow G$ be a monomorphism from $S$ into a compact topological group $G$. Then $h$ extends to a continuous homomorphism $h:(\beta S, \square) \rightarrow G$. We have $h(u)=e_{G}(u \in F)$, and so it follows that $h\left(s^{-1} F\right)=\left\{h(s)^{-1}\right\} \quad(s \in S)$. This shows that the family $\left\{s^{-1} F: s \in S\right\}$ is pairwise disjoint; each set $s^{-1} F$ has measure $\alpha$, and this is a contradiction unless $\alpha=0$.

Proposition 9.18. Let $S$ be an infinite, weakly left cancellative, left-amenable semigroup. Then, for each $\alpha \geq 1$, $\Im\left(M_{1}(\beta S)\right)$ has minimal elements of norm $\alpha$.

Proof. By Proposition 6.23(i), there are minimal left ideals $L_{1}$ and $L_{2}$ in $(\beta S, \square)$ with $L_{1} \cap L_{2}=\emptyset$. By Proposition 9.16(i), there are $\mu_{1}, \mu_{2} \in \mathfrak{L}(S)$ such that $\operatorname{supp} \mu_{k}=L_{k}(k=1,2)$. For $\alpha_{1}, \alpha_{2} \in \mathbb{C}$ with $\alpha_{1}+\alpha_{2}=1$, set $\nu=\alpha_{1} \mu_{1}+\alpha_{2} \mu_{2}$. Then $\nu \in \mathfrak{I}_{1}(M(\beta S)), \nu$ is minimal, and $\|\nu\|=\left|\alpha_{1}\right|+\left|\alpha_{2}\right|$, which can take arbitrary values in $[1, \infty)$.

The following result is well-known.

LEMma 9.19. Let $S$ be an infinite, left-amenable semigroup, and let $\mu \in \mathfrak{L}(S)$. Let $B$ be a Borel subset of $\beta S$ with $\mu(B)>0$. Then

$$
\left\{s \in S: \mu\left(s^{-1} B \cap B\right)>0\right\}
$$

belongs to $q$ for each $q \in E(\beta S)$.

Proof. Let $q \in E(\beta S)$, let $B$ be a Borel subset of $\beta S$ such that $\mu(B)>0$, and set

$$
A=\left\{s \in S: \mu\left(s^{-1} B \cap B\right)=0\right\} .
$$

Assume towards a contradiction that $A \in q$. By Proposition 6.8(ii), there exists $\left(s_{n}\right)$ in $S$ such that $F P\left\langle\left(s_{n}\right)\right\rangle \subset A$. Choose $n \in \mathbb{N}$ such that $n \mu(B)>1$. For $k, m \in \mathbb{N}$ with $k<m<n$, we have

$$
\begin{aligned}
\mu\left(\left(s_{k} s_{k-1} \cdots s_{2} s_{1}\right)^{-1} B\right. & \left.\cap\left(s_{m} s_{m-1} \cdots s_{2} s_{1}\right)^{-1} B\right) \\
= & \mu\left(B \cap\left(s_{m} s_{m-1} \cdots s_{k+1}\right)^{-1} B\right)=0 .
\end{aligned}
$$


It follows that

$$
\mu\left(\bigcup_{m=1}^{n}\left(s_{m} s_{m-1} \cdots s_{2} s_{1}\right)^{-1} B\right) \geq n \mu(B)>1,
$$

a contradiction. Thus $S \backslash A \in q$, giving the result.

THEOREM 9.20. Let $S$ be an infinite, left-amenable semigroup, and let $\mu \in \mathfrak{L}(S)$. Let $K$ be a compact subset of $\beta S$ with $\mu(K)>0$, let $q \in E(\beta S)$, and let $Q \in q$. Then there exists $p \in E(\beta S) \cap \bar{Q}$ such that $K \cap p K \neq \emptyset$.

Proof. Set $T=\left\{s \in S: \mu\left(s^{-1} K \cap K\right)>0\right\}$. By Lemma 9.19, $E(\beta S) \subset \bar{T}$.

We shall find $p \in E(\beta S) \cap \bar{Q}$ and $x \in K$ such that $p x \in K$. This is sufficient to prove the theorem. We may suppose that $Q \subset T$.

By Proposition 6.10(i), we have $Q^{\star}(q) \in q$ : choose $t_{1} \in Q^{\star}(q)$, so that $\mu\left(t_{1}^{-1} K \cap K\right)>0$, and then set $K_{1}=t_{1}^{-1} K \cap K$ and

$$
Q_{1}=\left\{s \in t_{1}^{-1} Q^{\star}(q): \mu\left(s^{-1} K_{1} \cap K_{1}\right)>0\right\} .
$$

By Proposition 6.10(ii), $t_{1}^{-1} Q^{\star}(q) \in q$ and $\left\{s \in S: \mu\left(s^{-1} K_{1} \cap K_{1}\right)>0\right\}$ belongs to $q$ by Lemma 9.19 , and so $Q_{1} \in q$, whence $Q_{1}^{\star}(q) \in q$.

Next choose $t_{2} \in Q_{1}^{\star}(q)$, so that

$$
\mu\left(t_{2}^{-1} t_{1}^{-1} K \cap t_{2}^{-1} K \cap t_{1}^{-1} K \cap K\right)>0 .
$$

Continuing in this way, we find a subset $\left\{t_{n}: n \in \mathbb{N}\right\}$ of $S$ such that $F P\left\langle\left(t_{n}\right)\right\rangle \subset Q$ and

$$
\mu\left(\bigcap\left\{t^{-1} K \cap K: t \in F P\left\langle\left(t_{1}, \ldots, t_{n}\right)\right\rangle\right\}\right)>0
$$

for each $n \in \mathbb{N}$. Since $\left\{t^{-1} K \cap K: t \in F P\left\langle\left(t_{n}\right)\right\rangle\right\}$ is a family of nonempty, compact subsets of $\beta S$ with the finite intersection property, there exists $x \in \bigcap\left\{t^{-1} K \cap K: t \in F P\left\langle\left(t_{n}\right)\right\rangle\right\}$. By Proposition 6.8(i), there exists $p \in E(\beta S)$ such that

$$
p \in \overline{F P\left\langle\left(t_{n}: n \geq m\right)\right\rangle} \quad(m \in \mathbb{N}),
$$

and hence $p \in \bar{Q}$. Since $t x \in K$ for each $t \in F P\left\langle\left(t_{n}\right)\right\rangle$, we have $p x \in K$, and so $p$ and $x$ have all the required properties.

THEOREM 9.21. Let $S$ be an infinite, left-amenable, cancellative semigroup. Then:

(i) $\overline{L(\beta S)} \subset \overline{E\left(S^{*}\right) \square S^{*}} \subset \overline{S_{[\infty]}^{*}}$;

(ii) in the case where $S$ is countable, $L(\beta S) \not \subset S_{[2]}^{*}$. 
Proof. (i) We have $E\left(S^{*}\right) \neq \emptyset$; take $q \in E\left(S^{*}\right)$. Set $U=\beta S \backslash\left\{e_{S}\right\}$ if $S$ has an identity $e_{S}$, and $U=\beta S$ otherwise.

Let $v \in L(\beta S)$, and take $A \in v$. Then there exists $\mu \in \mathfrak{L}(S)$ with $\mu(\bar{A})>0$. By Theorem 9.20, with $K=\bar{A}$ and $Q=U$, there exists $p \in E(\beta S) \cap U$ and $x \in \bar{A}$ such that $p x \in \bar{A}$. Since $p x \in E\left(S^{*}\right) \square S^{*}$, $v \in \overline{E\left(S^{*}\right) \square S^{*}}$, and so the result follows.

(ii) By Proposition 6.28, there is a sequence $\left(p_{n}\right)$ of idempotents in $K(\beta S)$ and $u \in \overline{\left\{p_{n}: n \in \mathbb{N}\right\}} \backslash S_{[2]}^{*}$. Each ideal $S^{*} p_{n}$ is a minimal left ideal. By Proposition 9.16(i), for each $n \in \mathbb{N}$, there is $\mu_{n} \in \mathfrak{L}(S)$ with $\operatorname{supp} \mu_{n}=S^{*} p_{n}$. Set $\mu=\sum_{n=1}^{\infty} \mu_{n} / 2^{n}$. Then $u \in \operatorname{supp} \mu \subset L(\beta S)$.

Theorem 9.22. Let $S$ be a weakly left cancellative, left-amenable semigroup. Then

$$
\overline{K(\beta S)} \subset \overline{L(\beta S)} \subset \overline{S_{[\infty]}^{*}} .
$$

Proof. This now follows from Proposition 9.16(ii) and Theorem 9.21 .

Means in $M(\beta \mathbb{N})$ We now make some remarks that are specific to the semigroup $(\mathbb{N},+)$.

For $n \in \mathbb{N}$, define $f_{n} \in \ell^{1}(\mathbb{N})_{[1]}$ by $f_{n}=\chi_{\mathbb{N}_{n}} / n$, and let $\mu \in M(\beta \mathbb{N})$ be a weak-* accumulation point of the sequence $\left(f_{n}\right)$. It is immediate that $\mu$ is a mean on $\mathbb{N}$, and then it is clear that $\mu$ is invariant, so that $\mu \in \mathfrak{L}(\mathbb{N})$. The set of means that arise is $\mathfrak{L}_{0}(\mathbb{N})$, and then

$$
L_{0}(\beta \mathbb{N})=\bigcup\left\{\operatorname{supp} \mu: \mu \in \mathfrak{L}_{0}(\mathbb{N})\right\} .
$$

Clearly $L_{0}(\beta \mathbb{N}) \subset L(\beta \mathbb{N})$.

We also give some notation taken from $[\mathbf{7 8}, \S 6.7]$.

Definition 9.23. Let $U$ be a subset of $\mathbb{N}$. Then the upper density of $U$ is

$$
\bar{d}(U)=\limsup _{n \rightarrow \infty} \frac{\left|U \cap \mathbb{N}_{n}\right|}{n},
$$

and

$$
\Delta=\{u \in \beta \mathbb{N}: \bar{d}(U)>0(U \in u)\} .
$$

A related concept which occurs frequently in ergodic theory is that of the upper Banach density $d^{*}(U)$

of a subset $U$ of $\mathbb{N}$. Indeed $d^{*}(U)$ is the supremum of the numbers $\alpha \in \mathbb{R}$ such that, for each $k \in \mathbb{N}$, there exist $m, n \in \mathbb{N}$ with $n>k$ such that

$$
\left|U \cap\left(m+\mathbb{N}_{n}\right)\right| \geq \alpha n .
$$


See $\left[\mathbf{7 8}\right.$, Chapter 20]. Note that $d^{*}(U) \geq \bar{d}(U)$. There is an extensive literature in ergodic and Ramsey theory about subsets $U$ of $\mathbb{N}$ (and other semigroups) with $d^{*}(U)>0$. A famous theorem in this context is that of Szemerédi [78, Corollary 20.14]:

each such set $U$ contains arbitrarily long arithmetical progressions.

Proposition 9.24. The subset $\Delta$ is a closed left ideal in $(\beta \mathbb{N}, \square)$, and the subset $\mathbb{N}^{*} \backslash \Delta$ is a left ideal in $(\beta \mathbb{N}, \square)$.

Proof. This is [78, Theorems 6.79 and 6.80].

THEOREM 9.25. In the above notation, $\Delta=\overline{L_{0}(\beta \mathbb{N})}$.

Proof. Let $u \in \Delta$. Take $U \in u$, and set $\delta=\bar{d}(U)$, so that $\delta>0$. There is a strictly increasing sequence $\left(n_{k}\right)$ in $\mathbb{N}$ such that

$$
2\left|U \cap \mathbb{N}_{n_{k}}\right|>\delta n_{k} \quad(k \in \mathbb{N})
$$

For $k \in \mathbb{N}$, let $f_{n_{k}}$ be as above, and let $\mu$ be an accumulation point of the set $\left\{f_{n_{k}}: k \in \mathbb{N}\right\}$, so that $\mu \in \mathfrak{L}_{0}(\mathbb{N})$. Since $f_{n_{k}}(U)>\delta / 2(k \in \mathbb{N})$, we have $\mu(\bar{U}) \geq \delta / 2$, and so $\bar{U} \cap \operatorname{supp} \mu \neq \emptyset$. Thus $u \in \overline{L_{0}(\beta \mathbb{N})}$.

Let $u \in \overline{L_{0}(\beta \mathbb{N})}$. For each $U \in u$, there exists $\mu \in \mathfrak{L}_{0}(\mathbb{N})$ such that $\mu(\bar{U})>0$, say $\delta=\mu(\bar{U})$. There is a strictly increasing sequence $\left(n_{k}\right)$ in $\mathbb{N}$ such that equation (9.3) holds, and so $\bar{d}(U) \geq \delta / 2>0$. Thus $u \in \Delta$.

Clause (i) of the next result also follows from [7, Theorem 2.6] (where different terminology is used). It was already shown in $[\mathbf{4 0}$, Proposition 3.4] that $K \beta S) \subsetneq L(\beta S)$ whenever $S$ is a semigroup containing a ' $C$-subset', which includes the case where $S=\mathbb{N}$.

Proposition 9.26. (i) The set $\Delta$ is not contained in $\overline{K(\beta \mathbb{N})}$.

(ii) $K(\beta \mathbb{N}) \not \subset \overline{L_{0}(\beta \mathbb{N})}$.

(iii) $L_{0}(\beta \mathbb{N}) \not \subset K(\beta \mathbb{N})$, and $\overline{K(\beta \mathbb{N})} \subsetneq \overline{L(\beta \mathbb{N})}$.

Proof. Set $K=K(\beta \mathbb{N}), L=L(\beta \mathbb{N})$, and $L_{0}=L_{0}(\beta \mathbb{N})$.

(i) A number $n \in \mathbb{N}$ is said to be square-free

if $n \notin p^{2} \mathbb{N}$ for each prime number $p$. Let $A$ be the set of square-free numbers. Then it is clear that $\bar{d}(A)>0$, and so $A^{*} \cap \Delta \neq \emptyset$. However, by [78, Theorem 4.40], we have $A^{*} \cap \bar{K} \neq \emptyset$. Hence $\Delta \not \subset \bar{K}$.

(ii) Since $N^{*} \backslash \Delta$ is a left ideal in $(\beta \mathbb{N}, \square)$, we have $\left(N^{*} \backslash \Delta\right) \cap K \neq \emptyset$. But $\Delta=\overline{L_{0}(\beta \mathbb{N})}$ by Theorem 9.25 , and so $K \not \subset \overline{L_{0}}$.

(iii) This follows immediately. 
We can now give an example that was promised on page 102.

Proposition 9.27. There exist $\mu \in \mathfrak{L}_{0}(\beta \mathbb{N})$ and $v \in \mathbb{N}^{*}$ such that

$$
\operatorname{supp}(v \square \mu) \not \subset \overline{v \square(\operatorname{supp} \mu)} \text {. }
$$

Proof. By Proposition 9.26 and Theorem 9.25, there exists a measure $\mu \in \mathfrak{L}_{0}(\beta \mathbb{N})$ such that $L \not \subset \overline{K(\beta \mathbb{N})}$, where $L=\operatorname{supp} \mu$. Take $v \in K(\beta \mathbb{N})$. Then $\operatorname{supp}(v \square \mu)=L$, and $v \square(\operatorname{supp} \mu) \subset K(\beta \mathbb{N})$ because $K(\beta \mathbb{N})$ is a right ideal. The result follows.

Proposition 9.28. The closed subspace $M(\overline{K(\beta \mathbb{N})})$ of $M(\beta \mathbb{N})$ is a left ideal, but not a right ideal, in $(M(\beta \mathbb{N}), \square)$.

Proof. By Proposition 6.15, $\overline{K(\beta \mathbb{N})}$ is an ideal in $\beta \mathbb{N}$. By Proposition 7.5(i), $M(\overline{K(\beta \mathbb{N})})$ is a left ideal. By Proposition 9.27, there exists $v \in K(\beta \mathbb{N})$ and $\mu \in M\left(\mathbb{N}^{*}\right)$ with $v \square \mu \notin M(\overline{K(\beta \mathbb{N})})$, and so $M(\overline{K(\beta \mathbb{N})})$ is not a right ideal.

Our final remark in this chapter is to show that there are often idempotents in $S^{*}$ which are not in $\overline{L(\beta S)}$.

THEOREM 9.29. Let $S$ be a infinite, cancellative, abelian semigroup. Then $E\left(S^{*}\right) \backslash \overline{L(\beta S)}$ is dense in $E\left(S^{*}\right)$.

Proof. By Proposition 3.16, there is a sequence $\left(s_{n}: n \in \mathbb{N}\right)$ in $S$ which has distinct finite products. Let $A$ be an infinite subset of $\mathbb{N}$ such that $\mathbb{N} \backslash A$ is infinite, so that $\left(s_{n}: n \in A\right)$ has distinct finite products; set $F=F P\left\langle\left(s_{n}: n \in A\right)\right\rangle$. For each pair $\left\{k_{1}, k_{2}\right\}$ of distinct elements of $\mathbb{N} \backslash A$, we have $s_{k_{1}} F \cap s_{k_{2}} F=\emptyset$ (using the fact that $S$ is abelian), and so $\left\{s_{k} \bar{F}: k \in \mathbb{N} \backslash A\right\}$ is an infinite family of pairwise disjoint sets in $\beta S$.

Let $\mu \in \mathfrak{L}(S)$. Then it follows that $\mu(F)=0$. Since $\bar{F}$ is an open subset of $\beta S$, it follows that $\bar{F} \cap \operatorname{supp} \mu=0$, and so $\bar{F} \cap \overline{L(\beta S)}=\emptyset$. By Proposition 6.8, there exists $p \in \bar{F} \cap E(\beta S)$. The result follows.

We now summarize some results in the special case where $S$ is the semigroup $(\mathbb{N},+)$; here

$$
L=L(\beta \mathbb{N})=\bigcup\{\operatorname{supp} \mu: \mu \in \mathfrak{L}(\mathbb{N})\}
$$

and $K=K(\beta \mathbb{N})$, the minimum ideal of $(\beta \mathbb{N}, \square)$. 
TheOrem 9.30. (i) $L$ and $\bar{L}$ are ideals in $(\beta \mathbb{N}, \square)$.

(ii) $K \subsetneq L$ and $\bar{K} \subsetneq \bar{L} \subset \overline{\mathbb{N}_{[\infty]}^{*}}$.

(iii) $L \not \subset \mathbb{N}_{[2]}^{*}$ and $\bar{L} \not \subset E\left(\mathbb{N}^{*}\right)$.

The main point that we have left open is the following.

Question Is $L(\beta \mathbb{N})$ closed in $\beta \mathbb{N}$ ? 

CHAPTER 10

\section{Amenability of semigroup algebras}

There is an extensive literature studying the amenability and weak amenability of Banach algebras on locally compact groups and on semigroups.

In this chapter, we shall discuss the amenability of the semigroup algebras $\ell^{1}(S)$ for a semigroup $S$.

Locally compact groups Let us first recall the known theory of the amenability of Banach algebras on locally compact groups $G$. The results reveal that conditions for the amenability and weak amenability of the group algebra

$L^{1}(G)$ and the measure algebra $M(G)$

are known; they also concern the Fourier algebra $A(G)$.

The first result combines two famous theorems of B. E. Johnson $[83],[85],[30]$.

THEOREM 10.1. Let $G$ be a locally

compact group.

(i) $L^{1}(G)$ is an amenable Banach algebra if and only if $G$ is an amenable

group.

(ii) $L^{1}(G)$ is weakly amenable.

Clause (i) of the above theorem is extended in the next theorem. Recall that the notions of a $C$-amenable

Banach algebra $A$ and of the amenability constant,

$A M(A)$, of $A$ were defined in Definition 2.3.

THEOREM 10.2. Let $G$ be a locally compact group. Then the following are equivalent:

(a) $G$ is an amenable

group;

(b) $L^{1}(G)$ is amenable;

(c) $A M\left(L^{1}(G)\right)=1$;

(d) $L^{1}(G)$ is approximately

amenable. 
Proof. The equivalence of (a) and (b) was first proved in [83]; see [19, Theorem 5.6.42]. That (b) implies (c) was explicitly given by Stokke [131, Corollary 1.10], and the converse is immediate. The equivalence of (a) and (d) is given in [51, Theorem 3.2].

Corollary 10.3. Let $G$ be an amenable group, and let $n \in \mathbb{N}$. Then $A M\left(\mathbb{M}_{n}\left(\ell^{1}(G)\right)=n\right.$.

Proof. Since $A M\left(\ell^{1}(G)\right)=1$ and $\ell^{1}(G)$ has an identity, this follows from Theorem 2.7(iv).

Clauses (i) and (iii) of the following theorem were proved by Dales, Ghahramani, and Helemskii in [23]; clause (ii) is [51, Theorem 3.1].

THEOREM 10.4. Let $G$ be a locally compact group.

(i) $M(G)$ is amenable

if and only if $G$ is discrete and amenable.

(ii) $M(G)$ is approximately amenable

if and only if it is amenable.

(iii) The following are equivalent: (a) $G$ is discrete; (b) there is no non-zero, continuous point derivation at the discrete augmentation character on $M(G)$; (c) $M(G)$ is weakly amenable.

Fourier algebras We give one further known example involving amenability and amenability constants.

Let $G$ be a locally compact group, and let $A(G)$ be the Fourier algebra

of $G$. This algebra was introduced by Eymard [39], and has been much studied. See [19, Definition 4.5.29] for a definition of $A_{p}(G)$ for $1<p<\infty$; we have $A(G)=A_{2}(G)$. When $G$ is abelian, $A(G)$ is isometrically isomorphic to $L^{1}(\Gamma)$, where $\Gamma$ is the dual group of $G$. Now let $\Gamma$ be the dual object of $G$, so that $\Gamma$ is the collection of all (equivalence classes of) continuous, irreducible, unitary representations of $G$ on a Hilbert space; for $\pi \in \Gamma$, let $d_{\pi}$ be the degree of $\pi$, the dimension of the corresponding Hilbert space.

In the case where $G$ is finite, $A(G)$ is amenable, and the amenability constant

is calculated by Johnson in [86]. Indeed, [86, Theorem 4.1] shows the remarkable formula that

$$
A M(A(G))=\frac{\sum\left\{d_{\pi}^{3}: \pi \in \Gamma\right\}}{\sum\left\{d_{\pi}^{2}: \pi \in \Gamma\right\}} .
$$


This constant is a rational number. For example, it is shown by Runde in $[\mathbf{1 2 6}]$ that, in the case where $G=A_{5}$, the alternating group on 5 letters, we have

$$
A M\left(A\left(A_{5}\right)\right)=61 / 15 .
$$

We see that $A M(A(G))=1$ if and only if $G$ is abelian; for $G$ nonabelian, $A M(A(G)) \geq 3 / 2[\mathbf{8 6}$, Proposition 4.3], and so the interval $(1,3 / 2)$ consists of 'forbidden values' for the constant $A M(A(G))$ when $G$ is a finite group. Let $n \in \mathbb{N}$. Then it is shown in [100, Theorem 4.9] that necessarily $\sup \left\{d_{\pi}: \pi \in \gamma\right\} \leq n^{2}$ whenever $2 A M(A(G))<n$.

Let $G$ be an infinite locally compact group such that

$$
\left\{\pi \in \Gamma: d_{\pi}=n\right\}
$$

is finite for each $n \in \mathbb{N}$. Then Johnson showed that $A(G)$ is not amenable. For example, $A(S O(3))$ is not amenable.

Let $G$ be a locally compact group.

Then it follows easily that $A(G)$ is amenable whenever $G$ is almost abelian, in the sense that $G$ has an abelian subgroup of finite index [100, Theorem 4.1]. The converse of this statement is proved by Forrest and Runde in [48, Theorem 2.3]. It is proved in [126] that $A M(A(G))=1$ if and only if $G$ is abelian; the same result is established for the related algebras $A_{p}(G)$ in [127] whenever $1<p<\infty$. We remark that it is also shown in $[48]$ that $A(G)$ is weakly amenable if and only if the connected component of the identity in $G$ is abelian.

It will be seen in this chapter that the number 5 places a role in the calculation of amenability constants; it is interesting to note that the same number arises in the context of the 'operator $C$-amenability'

of Fourier-Stieltjes algebras; see [128] and [129, Theorem 2.3].

Semigroup algebras Now that characterizations of the groups such that $L^{1}(G)$ is (weakly) amenable are known, it is natural to ask when a semigroup algebra $\ell^{1}(S)$ is (weakly) amenable. There are several known partial results, which we summarize in the following theorem. The force of these results seems to be that $\ell^{1}(S)$ is amenable if and only if $S$ is 'built up from amenable groups'; we shall resolve the question when $\ell^{1}(S)$ is amenable by showing exactly how $S$ is built up from amenable groups in this case.

For an early survey on when a semigroup algebra is amenable, see $[\mathbf{9 3}]$; a key paper for us is [33].

Proposition 10.5. Let $S$ be a semigroup.

(i) Suppose that $S$ is abelian and $E(S)=S$. Then $\ell^{1}(S)$

is weakly amenable. 
(ii) Suppose that $\ell^{1}(S)$ is an amenable Banach algebra. Then: $S$ is an amenable semigroup; $S$ has only finitely many idempotents, and $S$ has a minimal idempotent; each ideal $I$ in $S$ is a regular semigroup and, in particular, $I=I^{[2]} ; \ell^{1}(S)$ is a semisimple algebra.

(iii) Suppose that $S$ is unital and left or right cancellative. Then $\ell^{1}(S)$ is amenable if and only if $S$ is an amenable group.

(iv) Suppose that $S$ is abelian. Then $\ell^{1}(S)$ is amenable

if and only if $S$ is a finite semi-lattice of amenable groups.

Proof. (i) For each semigroup $S$ with $E(S)=S, \ell^{1}(S)$ is spanned by its idempotents, and so this follows from Proposition 2.2(v).

(ii) Let $I$ be an ideal in $S$. Then $\ell^{1}(I)$ is a closed ideal in $\ell^{1}(S)$, and it is complemented as a Banach space. By Proposition 2.2(vii), $\ell^{1}(I)$ is an amenable Banach algebra. It is proved in [32] that $S$ and $I$ are amenable, and in $[33]$ that $I$ is regular and that $E(I)$ is finite. The fact that $\ell^{1}(S)$ is semisimple now follows from [37, Theorem 5.11].

(iii) This is [67, Theorem 2.3].

(iv) This is a theorem of Grønbæk [68].

In fact, it can be shown that $\ell^{1}(S)$ is 'left-amenable' if and only if $S$ is a left-amenable semigroup [91].

The following corollary is at least implicit in [33] and [37].

COROLlary 10.6. Let $S$ be a semigroup such that $\ell^{1}(S)$ is amenable. Then:

(i) $\ell^{1}(S)$ has an identity;

(ii) $K(S)$ exists and is an amenable group.

Proof. (i) Since $\ell^{1}(S)$ is amenable, $\ell^{1}(S)$ has a bounded approximate identity. By Proposition 10.5(ii), $S$ is regular, and so, by (3.1),

$$
S=\bigcup\{p S q: p, q \in E(S)\} .
$$

By Proposition 10.5(ii), the set $E(S)$ is finite, and so it follows from Proposition 4.3 that $\ell^{1}(S)$ has an identity.

(ii) By Proposition 10.5, $S$ is regular and has only finitely many idempotents. By the structure theorem 3.12, $K(S)$ exists as an ideal in $S$. Since $S$ is an amenable semigroup, the semigroup $K(S)$ is also regular and amenable; certainly $E(K(S))$ is finite and $K(S)$ is simple. The result now follows from Proposition 9.9.

Let $S$ be a semigroup such that $\ell^{1}(S)$ is weakly amenable. Then certainly $S=S^{2}$. We do not know of an abelian semigroup $S$ such that $\ell^{1}(S)$ is weakly amenable, but $S$ is not regular. 
The weak amenability of $\ell^{1}(S)$ is considered by Blackmore in [11]. It is shown that $\ell^{1}(S)$ is weakly amenable whenever $S$ is completely regular,

in the sense that, for each $s \in S$, there exists $t \in S$ with $s t s=s$ and $s t=t s$.

EXAMPLE 10.7. Let $S$ be an infinite set which is a right zero semigroup.

As in Example 4.11, $f \star g=\varphi_{S}(f) g\left(f, g \in \ell^{1}(S)\right)$, and so it follows from Proposition 2.13 that $\ell^{1}(S)$ is weakly amenable, but not amenable.

ExAmPLE 10.8. Let $S$ be a semigroup, and let $S^{o}$ be the semigroup formed by adjoining a zero $o$ to $S$. Then clearly $\ell^{1}\left(S^{o}\right)=\ell^{1}(S) \oplus \mathbb{C} \delta_{o}$ as a Banach space. The product in $\ell^{1}\left(S^{o}\right)$ is such that

$$
f \star \delta_{o}=\delta_{o} \star f=\varphi_{S}(f) \delta_{o} \quad\left(f \in \ell^{1}(S)\right),
$$

and so $\ell^{1}\left(S^{o}\right)=\ell^{1}(S) \ltimes \mathbb{C} \delta_{o}$. Assume that $\ell^{1}\left(S^{o}\right)$ is [weakly] amenable. Then it follows from Proposition 2.2, (vii) and $(\mathrm{x})$, that $\ell^{1}(S)$ is also [weakly] amenable.

EXAMPLE 10.9. Let $T=(\mathbb{Z},+) \times S$ be the semigroup of Example 3.32 , where $S$ is the right zero semigroup on $\mathbb{N}$. Then $T$ has infinitely many idempotents, and so $\ell^{1}(T)$ is not amenable by Proposition 10.5(ii). (In fact, $T$ is left-amenable, but it is not right-amenable because it has disjoint minimal left ideals $\mathbb{Z} \times\{n\}$; see Proposition 9.8(ii).) Since $\mathbb{Z}$ is an abelian group, $\ell^{1}(\mathbb{Z})$ is a unital, commutative, amenable algebra; by Proposition $2.14, \ell^{1}\left(\mathbb{Z} \times S^{\#}\right)$ is weakly amenable.

ExAmple 10.10. Let $S$ be $\mathbb{N}_{\vee}$ or $\mathbb{N}_{\wedge}$, as in Examples 3.36 and 3.37; the semigroup algebras $\ell^{1}(S)$ were described in Examples 4.9 and 4.10, respectively. Clearly $S$ is abelian and $E(S)=S$, and so, by Proposition 10.5, (i) and (ii), $\ell^{1}(S)$ is weakly amenable, but not amenable.

Let $S=\mathbb{N}_{\wedge}$. Then we claim that $A=\ell^{1}(S)$ is approximately amenable;

we shall apply the criterion of Proposition 2.10. The details follow a remark of Rick Loy, to whom we are grateful. Indeed, for $n \in \mathbb{N}$, set

$$
F_{n}=\delta_{n} \otimes \delta_{n}+\sum_{j=1}^{n}\left(\delta_{j}-\delta_{j-1}\right) \otimes\left(\delta_{j}-\delta_{j-1}\right) \in A \widehat{\otimes} A,
$$


where $\delta_{0}=0$, and set $u_{n}=\delta_{n}$. Certainly $\pi\left(F_{n}\right)=2 u_{n}$ and $\lim _{n} a u_{n}=a$ for each $a \in A$. We check that

$$
\delta_{k} \cdot F_{n}-F_{n} \cdot \delta_{k}+\delta_{n} \otimes \delta_{k}-\delta_{k} \otimes \delta_{n}=0 \quad(k<n)
$$

and that

$$
\delta_{k} \cdot F_{n}=F_{n} \cdot \delta_{k}=F_{n} \quad(k \geq n) .
$$

Take $a=\sum_{k=1}^{\infty} \alpha_{k} \delta_{k} \in A$ and $n \in \mathbb{N}$, and set $b=\sum_{k=n}^{\infty} \alpha_{k} \delta_{k} \in A$. Then

$$
b \cdot F_{n}=F_{n} \cdot b=\left(\sum_{k=n}^{\infty} \alpha_{k}\right) F_{n}
$$

and so

$$
\begin{aligned}
\left\|a \cdot F_{n}-F_{n} \cdot a+u_{n} \otimes a-a \otimes u_{n}\right\|_{\pi} & =\left\|\delta_{n} \otimes b-b \otimes \delta_{n}\right\|_{\pi} \\
& \leq 2 \sum_{k=n}^{\infty}\left|\alpha_{k}\right| .
\end{aligned}
$$

Thus $\left\|a \cdot F_{n}-F_{n} \cdot a+u_{n} \otimes a-a \otimes u_{n}\right\|_{\pi} \rightarrow 0$ as $n \rightarrow \infty$. We have shown that the conditions in Proposition 2.10 are satisfied, and hence $A$ is approximately amenable.

Let $S=\mathbb{N}_{\vee}$. Then we again claim that $A=\ell^{1}(S)$ is approximately amenable. Indeed, for $n \in \mathbb{N}$, set

$$
F_{n}=2 \delta_{1} \otimes \delta_{1}-\sum_{j=1}^{n}\left(\delta_{j} \otimes \delta_{j+1}+\delta_{j+1} \otimes \delta_{j}-2 \delta_{j+1} \otimes \delta_{j+1}\right) \in A \otimes A,
$$

and set $u=\delta_{1}$, the identity of $A$. Certainly $\pi_{A}\left(F_{n}\right)=2 u(n \in \mathbb{N})$. Set

$$
\Delta_{k}=\delta_{k} \cdot F_{n}-F_{n} \cdot \delta_{k}+\delta_{1} \otimes \delta_{k}-\delta_{k} \otimes \delta_{1} \quad(k \in \mathbb{N}) .
$$

Then $\Delta_{1}=0$. Also, for $k \in\{2, \ldots, n\}$, we have

$$
\Delta_{k}=\delta_{k} \otimes \delta_{1}-\delta_{1} \otimes \delta_{k}+\sum_{j=1}^{k-1}\left(\delta_{k} \otimes \delta_{j+1}-\delta_{k} \otimes \delta_{j}\right)+\sum_{j=1}^{k-1}\left(\delta_{j} \otimes \delta_{k}-\delta_{j+1} \otimes \delta_{k}\right)=0 \text {. }
$$

Similarly, for $k \geq n+1$, we have $\Delta_{k}=\delta_{k} \otimes \delta_{n+1}-\delta_{n+1} \otimes \delta_{k}$.

Take $a=\sum_{k=1}^{\infty} \alpha_{k} \delta_{k} \in A$ and $n \in \mathbb{N}$. Then, as above,

$$
\left\|a \cdot F_{n}-F_{n} \cdot a+\delta_{1} \otimes a-a \otimes \delta_{1}\right\|_{\pi} \leq 2 \sum_{k=n}^{\infty}\left|\alpha_{k}\right| \rightarrow 0 \quad \text { as } \quad n \rightarrow \infty .
$$

We have shown that the criteria for the approximate amenability of $A$ given in Proposition 2.10 are satisfied. This gives the claim.

We have no characterization of the semigroups $S$ such that $\ell^{1}(S)$ is approximately amenable. 
ExAmple 10.11. Let $S=\mathcal{M}^{o}(G, P, m, n)$ be a regular Rees matrix semigroup

with a zero over a group $G$. As in Chapter 4, we have

$$
\ell^{1}(S)=\mathcal{M}^{o}\left(\ell^{1}(G), P, m, n\right)=\mathcal{M}\left(\ell^{1}(G), P, m, n\right) \oplus \mathbb{C} \delta_{o}
$$

isometrically as a Banach space, with the product $\star_{P}$ specified in equations (4.7) and (4.8), where $G=p S p \backslash\{o\}$ is a group for each primitive idempotent $p \in E(S)$. Thus

$$
B:=\ell^{1}(S) / \mathbb{C} \delta_{o}=\mathcal{M}\left(\ell^{1}(G), P, m, n\right)
$$

isometrically and isomorphically.

In each such case, $\ell^{1}(S)$ is weakly amenable [11, Corollary 5.3].

Now suppose that $\ell^{1}(S)$ is amenable. Then $B$ is amenable. By Corollary 10.6(i), $\ell^{1}(S)$ has an identity, and so $\mathcal{M}\left(\ell^{1}(G), P, m, n\right)$ has an identity. As in Proposition 2.16, it follows that $m=n$, that $P$ is invertible in $\mathbb{M}_{n}\left(\ell^{1}(G)\right)$ with inverse $Q$, and that the map

$$
\theta: a \rightarrow Q a, \quad \mathbb{M}_{n}\left(\ell^{1}(G)\right) \rightarrow \mathcal{M}\left(\ell^{1}(G), P, n\right),
$$

is a topological isomorphism with $\|\theta\|=\|Q\|$. Thus $\mathbb{M}_{n}\left(\ell^{1}(G)\right)$ is amenable, and so $\ell^{1}(G)$ is amenable by Theorem 2.7(i); finally, this implies that the group $G$ is amenable.

Conversely, suppose that $G$ is amenable, that $m=n$, and that $P$ is invertible as a matrix in $\mathbb{M}_{n}\left(\ell^{1}(G)\right)$. Then $\ell^{1}(S) / \mathbb{C} \delta_{o}$ is topologically isomorphic to $\mathbb{M}_{n}\left(\ell^{1}(G)\right)$. Since $\ell^{1}(G)$ is 1 -amenable, it follows from the remarks after Proposition 2.16 that $\mathcal{M}\left(\ell^{1}(G), P, n\right)$ is $n v(P)^{2}$ amenable, where $v(P)=\max \left\{\sum_{r=1}^{n}\left\|\left(P^{-1}\right)_{r i}\right\|: i \in \mathbb{N}_{n}\right\}$. We have explained in Chapter 4 that we are in the situation of Example 2.11, and so, by Theorem 2.12(ii), $\ell^{1}(S)=\mathcal{M}^{o}\left(\ell^{1}(G), P, n\right)$ is $C$-amenable, where

$$
C=4 n v(P)^{2}+1 \text {. }
$$

In particular, we have shown that $\mathcal{M}^{o}\left(\ell^{1}(G), P, n\right)$ is an amenable Banach algebra if and only if $G$ is an amenable group, $m=n$, and $P$ is invertible as a matrix in $\mathbb{M}_{n}\left(\ell^{1}(G)\right)$.

We now determine exactly when a semigroup algebra is amenable. The result is conjectured in the last paragraph of [33], with reference to [32] for the method. The authors of [33] remark that they could prove the conjecture if they could achieve a certain technical step. Thus they require to know that $\ell^{1}(J)$ has a bounded approximate identity in the case where $\ell^{1}(S)$ is amenable and $J$ is an ideal of $S$. However in this case $\ell^{1}(J)$ is a closed, complemented ideal in the Banach algebra $\ell^{1}(S)$, and so $\ell^{1}(J)$ is amenable, and hence has a bounded approximate 
identity by Proposition 2.2(ii); indeed, by Corollary $10.6, \ell^{1}(J)$ has an identity.

THEOREM 10.12. Let $S$ be a semigroup. Then the Banach algebra $\ell^{1}(S)$ is amenable

if and only if the minimum ideal $K(S)$ exists, $K(S)$ is an amenable group, and $S$ has a principal series

$$
S=I_{1} \supsetneq I_{2} \supsetneq \cdots \supsetneq I_{m-1} \supsetneq I_{m}=K(S)
$$

such that each quotient $I_{j} / I_{j+1}$ is a regular Rees matrix semigroup

of the form $\mathcal{M}^{o}(G, P, n)$, where $n \in \mathbb{N}, G$ is an amenable group, and the sandwich matrix $P$ is invertible in $\mathbb{M}_{n}\left(\ell^{1}(G)\right)$.

Proof. Suppose that $\ell^{1}(S)$ is amenable. By Propositon 10.5(ii), $S$ is regular and $E(S)$ is finite, and so the structure theorem

3.12 applies. By Corollary 10.6(ii), $K(S)$ is an amenable group. Each quotient $I_{j} / I_{j+1}$ is a completely $o$-simple, regular semigroup with finitely many idempotents, and so, by Theorem 3.13, has the form $T=\mathcal{M}^{o}(G, P, m, n)$ for some $m, n \in \mathbb{N}$, a group $G$, and a sandwich matrix $P$. By Proposition 2.2(vii), $\ell^{1}(T)$ is amenable, and so, as in Example 10.11, $T$ has the specified form.

Conversely, suppose that $S$ has the specified form. Then $\ell^{1}(K(S))$ is amenable by Theorem 10.1(i), and $\ell^{1}(T)$ is amenable for each quotient $T=I_{j} / I_{j+1}$, as above. Hence $\ell^{1}(S)$ is amenable by Proposition $2.2(\mathrm{vi})$.

The example given in [37, Example 6.3] is not a counter-example to the conjecture of Duncan and Paterson, despite the remark on $[\mathbf{3 7}$, p. 365], because the specifed matrix $P$ is invertible in $\mathbb{M}_{2}\left(\ell^{1}(G)\right)$.

\section{Some calculations}

EXAMPLE 10.13. We give some specific examples of finite-dimensional, amenable semigroup algebras $A=\mathcal{M}^{o}(\mathbb{C}, P, n)$, and calculate $\left\|e_{A}\right\|_{1}$. So far we have shown that $\left\|e_{A}\right\|_{1} \geq 2 n-1$, and that all the values $2 n-1$ can be attained.

We again identify $P$ and $Q=P^{-1}$ with the numerical matrices $\left(\alpha_{i j}\right)$ and $\left(\beta_{i j}\right)$, respectively.

Take

$$
P=\left(\begin{array}{llll}
0 & 0 & 0 & 1 \\
0 & 1 & 1 & 0 \\
1 & 0 & 1 & 0 \\
1 & 1 & 0 & 1
\end{array}\right), \quad R=\left(\begin{array}{rrrr}
-1 & -1 & 1 & 1 \\
-1 & 1 & -1 & 1 \\
1 & 1 & 1 & -1 \\
2 & 0 & 0 & 0
\end{array}\right)
$$


so that $P R=2 I_{4} \in \mathbb{M}_{4}$ and $Q=P^{-1}=(1 / 2) R$. In this case, we have $\left\|e_{A}\right\|_{1}=14 / 2+|1-4 / 2|=8$, the smallest even integer that can be attained.

Next take

$$
P=\left(\begin{array}{lllll}
1 & 1 & 1 & 1 & 0 \\
1 & 1 & 0 & 0 & 1 \\
1 & 0 & 1 & 0 & 0 \\
1 & 0 & 0 & 1 & 1 \\
0 & 0 & 1 & 0 & 1
\end{array}\right), \quad R=\left(\begin{array}{rrrrr}
-1 & 1 & 3 & 1 & -2 \\
2 & 2 & -2 & -2 & 0 \\
1 & -1 & 1 & -1 & 2 \\
2 & -2 & -2 & 2 & 0 \\
-1 & 1 & -1 & 1 & 2
\end{array}\right)
$$

so that $P R=4 I_{5} \in \mathbb{M}_{5}$ and $Q=(1 / 4) R$. Then $\|Q\|=36 / 4=9$ and $\sum_{i, j=1}^{n} \beta_{i j}=3 / 2$, so that, by (4.10), $\left\|e_{A}\right\|_{1}=19 / 2>2 \cdot 5-1$; in particular, $\left\|e_{A}\right\|_{1}$ is not a natural number. It can be shown by numerical calculation that $19 / 2$ is the smallest non-integer that can be obtained with $G=\{e\}$.

EXAMPLE 10.14. We give an example of an algebra $A=\ell^{1}(S)$ such that $\left\|e_{A}\right\|_{1}$ takes the smallest non-integral value that we have found, namely $25 / 3$. Our algebra $A$ is amenable.

Indeed, take $G=\mathbb{Z}_{2}=\{0,1\}$, with $1+1=0$, and consider the semigroup $S=\mathcal{M}^{o}\left(\mathbb{Z}_{2}, P, 3\right)$, where $P$ is the sandwich matrix

$$
P=\left(\begin{array}{ccc}
0 & o & 0 \\
1 & 0 & o \\
1 & 1 & 0
\end{array}\right) \in \mathbb{M}_{3}\left(\mathbb{Z}_{2}^{o}\right)
$$

so that $S$ is a regular Rees matrix semigroup

with a zero over the group $\left(\mathbb{Z}_{2},+\right)$. The corresponding matrix in $\mathbb{M}_{3}\left(\ell^{1}\left(\mathbb{Z}_{2}\right)\right)$ is

$$
P=\left(\begin{array}{lll}
e & 0 & e \\
a & e & 0 \\
a & a & e
\end{array}\right)
$$

where we are writing $e=\delta_{0}$ and $a=\delta_{1}$. The determinant of this matrix $P$ is $\Delta=2 e-a \in \ell^{1}\left(\mathbb{Z}_{2}\right)$, with inverse $\Delta^{-1}=(2 e+a) / 3$. The inverse $Q$ of $P$ is

$$
\Delta^{-1}\left(\begin{array}{ccc}
e & a & -e \\
-a & e-a & a \\
e-a & -a & e
\end{array}\right)=\frac{1}{3}\left(\begin{array}{ccc}
2 e+a & e+2 a & -2 e-a \\
-e-2 a & e-a & e+2 a \\
e-a & -e-2 a & 2 e+a
\end{array}\right),
$$

and so $\varphi_{G}(Q)=1$. Thus $e_{A}=Q$ and $\left\|e_{A}\right\|_{1}=25 / 3$. 
EXAmPle 10.15. We present an example of a semigroup $S$ such that $A=\ell^{1}(S)$ is amenable, but supp $e_{A}$ is not finite.

Indeed, we set $S=\mathcal{M}^{o}(\mathbb{Z}, P, 3)$, where $P$ is the sandwich matrix

$$
P=\left(\begin{array}{lll}
0 & 0 & o \\
1 & 0 & 1 \\
o & 0 & 0
\end{array}\right) \in \mathbb{M}_{3}\left(\mathbb{Z}^{o}\right),
$$

so that $S$ is a regular Rees matrix semigroup

with a zero over the group $(\mathbb{Z},+)$. The corresponding matrix in $\mathbb{M}_{3}\left(\ell^{1}(\mathbb{Z})\right)$ is

$$
P=\left(\begin{array}{lll}
e & e & 0 \\
a & e & a \\
0 & e & e
\end{array}\right)
$$

where we are writing $e=\delta_{0}$ and $a=\delta_{1}$. The determinant of this matrix $P$ is $\Delta=e-2 a \in \ell^{1}(\mathbb{Z})$, an element with inverse

$$
\Delta^{-1}=-\frac{1}{2} \delta_{-1}\left(\delta_{0}+\frac{1}{2} \delta_{-1}+\frac{1}{4} \delta_{-2}+\cdots\right) ;
$$

$\Delta^{-1}$ has infinite support in $\ell^{1}(\mathbb{Z})$ and $\left\|\Delta^{-1}\right\|_{1}=1$. The inverse of $P$ is

$$
Q=\Delta^{-1}\left(\begin{array}{ccc}
a-e & e & -a \\
a & -e & a \\
-a & e & a-e
\end{array}\right)
$$

and the identity of $A$ is $e_{A}=Q+2 \delta_{o}$. Clearly supp $e_{A}$ is infinite, and $\left\|e_{A}\right\|_{1}=9+2=11$ because $\left\|\Delta^{-1} \star(a-e)\right\|_{1}=1$

The amenability constant of a semigroup algebra We now explore some ideas related to the amenability constant of a semigroup algebra. It is convenient to begin with some new notation.

DEFINITION 10.16. Let $S$ be a semigroup such that $\ell^{1}(S)$ is amenable. Then $C_{S}$ is the amenability constant of $\ell^{1}(S)$ and $E_{S}=|E(S)|$ is the number of idempotents in $S$.

Let $S$ be a semigroup. We know from Proposition 10.5(ii) that $E_{S}$ is finite whenever $C_{S}$ is finite. We are interested in the relationship between the constants $C_{S}$ and $E_{S}$. For example, the argument of Duncan and Paterson in [33] shows that

$$
\sqrt{E_{S}} \leq C_{S}
$$

whenever $\ell^{1}(S)$ is amenable; however this is rather a weak estimate. We also seek the minimum value of $C_{S}$ which forces $S$ to be an amenable group; for example, it was shown by Stokke in [131, Theorem 3.2] that 
$S$ is a group whenever $C_{S}<2$. More generally, we seek to determine the values that $C_{S}$ can take (and 'forbidden values' that $C_{S}$ cannot take).

We note that, if $S$ and $T$ are semigroups such that $\ell^{1}(S)$ and $\ell^{1}(T)$ are isometrically isomorphic (so that $C_{S}=C_{T}$ ), then $S$ and $T$ are isomorphic as semigroups, and so $E_{S}=E_{T}$.

The first result deals with the case where $C_{S}=1$ or $E_{S}=1$; the result will be strengthened in Corollary 10.28.

Proposition 10.17. Let $S$ be a semigroup such that $\ell^{1}(S)$ is amenable. Then the following are equivalent: (a) $C_{S}=1$; (b) $E_{S}=1$; (c) $S$ is a group.

Proof. Since the Banach algebra $\ell^{1}(S)$ is amenable, it follows from Proposition 10.5(ii) that $S$ is regular and has a minimal idempotent. Suppose that $E_{S}=1$. Then $S$ is a group by Proposition 3.6(ii), and so (b) $\Rightarrow(\mathrm{c})$. Clearly $E_{S}=1$ whenever $S$ is a group, and so (c) $\Rightarrow(\mathrm{b})$. By Theorem 10.2, (c) $\Rightarrow(\mathrm{a})$, and (a) $\Rightarrow$ (c) by $[\mathbf{1 3 1}$, Theorem 3.2].

Proposition 10.18. Let $S=T^{o}$ be a semigroup formed by adjoining a zero to a semigroup $T$, and take $C$ with $1 \leq C<5$. Then $\ell^{1}(S)$ is not $C$-amenable.

Proof. We have noted in Example 4.7 that there is a contraction $\theta: \ell^{1}\left(S^{o}\right) \rightarrow \ell^{1}(D)$ and that the amenability constant of $\ell^{1}(D)$ is 5 . Hence the amenability constant of $\ell^{1}(S)$ is at least 5 .

Let $A=\ell^{1}(S)$. We recall that $\left(A \widehat{\otimes} A,\|\cdot\|_{\pi}\right)$ is isometrically isomorphic to $\left(\ell^{1}(S \times S),\|\cdot\|_{1}\right)$; we shall use this fact when we calculate the norm $\|u\|_{\pi}$ of some elements $u \in A \widehat{\otimes} A$ (cf. equation (1.1)).

Proposition 10.19. There is an infinite, completely 0-simple, regular semigroup $S$ such that $|\mathfrak{L}(S)|=1$, such that $C_{S}=5$, and such that $E_{S}=2$, so that $S$ is not a group.

Proof. We take $G=\mathbb{Z}$, and set $S=G^{o}=\mathbb{Z} \cup\{o\}$, so that $S$ is an infinite semigroup with a zero. Set $A=\ell^{1}(S)$.

For $n \in \mathbb{N}$, define

$$
u_{n}=\frac{1}{2 n+1} \sum_{j=-n}^{n}\left(\delta_{j}-\delta_{o}\right) \otimes\left(\delta_{-j}-\delta_{o}\right)+\delta_{o} \otimes \delta_{o} \in A \widehat{\otimes} A .
$$


Let $n \in \mathbb{N}$. We see the following. First, $\left\|u_{n}\right\|_{\pi}=5$. Second,

$$
\pi\left(u_{n}\right)=\frac{1}{2 n+1} \sum_{j=-n}^{n}\left(\delta_{0}-\delta_{o}\right)+\delta_{o}=\delta_{0},
$$

and so equation (2.2) is satisfied. Third, $\delta_{o} \cdot u_{n}=u_{n} \cdot \delta_{o}=\delta_{o} \otimes \delta_{o}$, and so $\delta_{o} \cdot u_{n}-u_{n} \cdot \delta_{o}=0$. Finally, for each $k \in \mathbb{Z}$, we have

$$
\delta_{k} \cdot\left(\delta_{o} \otimes \delta_{o}\right)=\left(\delta_{o} \otimes \delta_{o}\right) \cdot \delta_{k}
$$

and

$$
\delta_{k} \cdot u_{n}-u_{n} \cdot \delta_{k}=\frac{1}{2 n+1}\left(\sum_{j=-n+k}^{n+k}-\sum_{j=-n}^{n}\right)\left(\delta_{j}-\delta_{o}\right) \otimes\left(\delta_{-j+k}-\delta_{o}\right),
$$

so that $\left\|\delta_{k} \cdot u_{n}-u_{n} \cdot \delta_{k}\right\|_{\pi} \leq 8|k| /(2 n+1)$. It follows easily that

$$
\lim _{n \rightarrow \infty}\left\|a \cdot u_{n}-u_{n} \cdot a\right\|_{\pi}=0 \quad(a \in A),
$$

giving equation (2.1).

We have shown that $\left(u_{n}\right)$ is a bounded approximate diagonal for $A$ with $\left\|u_{n}\right\|_{\pi}=5(n \in \mathbb{N})$, and so $A$ is 5-amenable.

By Proposition 10.18, $A$ is not $C$-amenable for any $C<5$, and so $C_{S}=5$. Clearly $E_{S}=2$. Since $S$ has a zero, $|\mathfrak{L}(S)|=1$.

We are seeking to calculate $E_{S}$ and $C_{S}$ in the special case where $S=\mathcal{M}^{\circ}(G, P, n)$, a regular Rees matrix semigroup with a zero over a group $G$; here $n \in \mathbb{N}, G$ is amenable, and $P$ is a sandwich matrix which is invertible in $\mathbb{M}_{n}\left(\ell^{1}(G)\right)$, so that $\ell^{1}(S)$ is amenable. Recall from equation (3.5) that $E_{S}=|N(P)|+1$.

We first note a result essentially already given in Example 10.11, above; we also use Proposition 2.4(i) and equation (4.12).

TheOREM 10.20. Let $S$ be the Rees matrix semigroup $\mathcal{M}^{o}(G, P, n)$, as above. Then

$$
2 n-1 \leq\left\|e_{A}\right\|_{1} \leq C_{S} \leq 4 n v(P)^{2}+1,
$$

where $A=\ell^{1}(S)$ and $v(P)=\max \left\{\sum_{r=1}^{n}\left\|\left(P^{-1}\right)_{r j}\right\|: j \in \mathbb{N}_{n}\right\}$.

We shall now try to calculate $C_{S}$ more accurately for the semigroup $S=\mathcal{M}^{o}(G, P, n)$. In the following, all sums are taken over the set $\mathbb{N}_{n}$, unless we state otherwise. The first lemma is surely well-known.

Lemma 10.21. Let $n \in \mathbb{N}$ with $n \geq 2$. Then each diagonal 
for $\mathbb{M}_{n}$ has the form

$$
\sum_{j, k} x_{j k}\left(\sum_{i} E_{i j} \otimes E_{k i}\right),
$$

where $\sum_{j} x_{j j}=1$.

PRoOF. Let $u=\sum_{i, j, k, \ell} x_{i j k \ell} E_{i j} \otimes E_{k \ell}$ be a diagonal for $\mathbb{M}_{n}$, where each $x_{i j k \ell} \in \mathbb{C}$. For each $r \in \mathbb{N}_{n}$, we have $E_{r r} \cdot u=u \cdot E_{r r}$, and so $x_{i j k \ell}=0$ unless $i=\ell=r$. Thus we may suppose that

$$
u=\sum_{i, j, k} x_{i j k} E_{i j} \otimes E_{k i},
$$

where each $x_{i j k} \in \mathbb{C}$. Now take $r, s \in \mathbb{N}_{n}$ with $r \neq s$. Since

$$
E_{r s} \cdot u=\sum_{s, j, k} x_{s j k} E_{r j} \otimes E_{k s} \quad \text { and } \quad u \cdot E_{r s}=\sum_{r, j, k} x_{s j k} E_{r j} \otimes E_{k s},
$$

it follows that $x_{s j k}=x_{r j k}$ for each $j, k \in \mathbb{N}_{n}$, say the common value of $x_{s j k}$ for $s \in \mathbb{N}_{n}$ is $x_{j k}$. Thus $u$ has the specified form.

Since $\pi_{A}(u)=\sum_{j} x_{j j} \sum_{i} E_{i i}$, it follows that $\sum_{j} x_{j j}=1$.

We adopt the notation $\left(\mathcal{M}^{o}(\mathbb{C}, P, n), \star_{P}\right)$ and $\left(\mathcal{M}^{o}(\mathbb{C}, n), \star\right)$ from Definition 4.13 (in the case where $G=\{e\}$ ).

Lemma 10.22. Let $n \in \mathbb{N}$ with $n \geq 2$. Then each diagonal for $\left(\mathcal{M}^{o}(\mathbb{C}, n), \star\right)$ has the form

$$
\sum_{j, k} x_{j k}\left(\sum_{i} E_{i j} \otimes E_{k i}\right)+\delta_{o} \otimes \delta_{o},
$$

where $\sum_{j} x_{j j}=1, \sum_{j} x_{j k}=0\left(k \in \mathbb{N}_{n}\right)$, and $\sum_{k} x_{j k}=0\left(j \in \mathbb{N}_{n}\right)$.

Proof. Set $A=\mathcal{M}^{o}(\mathbb{C}, n)$ and $B=\mathbb{M}_{n}$, and identify $A$ with $B \oplus \mathbb{C} \delta_{o}$, as before. We temporarily denote the module actions in $A \otimes A$ and $B \otimes B$ by $\cdot A$ and $\cdot B$, respectively.

A diagonal $u$ for $A$ has the form

$$
u=v-y \otimes \delta_{o}-\delta_{o} \otimes z+w \delta_{o} \otimes \delta_{o},
$$

where $v$ is a diagonal for $B, y, z \in B$, and $w \in \mathbb{C}$. By Lemma 10.21, $v$ has the form specified in that lemma; in particular, $\sum_{j} x_{j j}=1$. Set $x=\sum_{i \neq j} x_{i j}$, so that $\sum_{i, j} x_{i j}=x+1$. Then

The projection of $A \otimes A$ onto $A \otimes B$ is denoted by $R$. Let $r, s \in \mathbb{N}_{n}$.

$$
R\left(E_{r s} \cdot A u\right)=E_{r s} \cdot B v+\sum_{j, k} x_{j k}\left(\sum_{i \neq s} \delta_{o} \otimes E_{k i}\right)-\delta_{o} \otimes z,
$$


and $R\left(u \cdot{ }_{A} E_{r s}\right)=v \cdot{ }_{B} E_{r s}$. It follows that

$$
z=\sum_{k}\left(\sum_{j} x_{j k}\right) \sum_{i \neq s} E_{k i} .
$$

This holds for each $s \in \mathbb{N}_{n}$; since $n \geq 2$, this implies that $z=0$ and $\sum_{j} x_{j k}=0\left(k \in \mathbb{N}_{n}\right)$. We now see that $x+1=0$.

Similarly, $y=0$ and $\sum_{k} x_{j k}=0\left(j \in \mathbb{N}_{n}\right)$. Thus our diagonal $u$ now has the form $u=v+w \delta_{o} \otimes \delta_{o}$.

We confirm that $\delta_{O} \cdot{ }_{A} u=u \cdot{ }_{A} \delta_{o}$, and so $a \cdot{ }_{A} u=u \cdot{ }_{A} a$ for each $a \in A$.

We know that

$$
\pi_{A}(u)=\pi_{B}(v)+n x \delta_{o}+w \delta_{0}=\sum_{i} E_{i i}+(w-n) \delta_{o} .
$$

But $\pi_{A}(u)=e_{A}=\sum_{i} E_{i i}-(n-1) \delta_{o}$. Hence $w=1$.

Thus our diagonal $u$ has the specified form.

We continue to take $G=\{e\}$. Let $P=\left(\alpha_{i j}\right) \in \mathbb{M}_{n}$ be an invertible sandwich matrix, so that each $\alpha_{i j} \in\{0,1\}$, and set $Q=P^{-1}=\left(\beta_{i j}\right)$, as before. Again all indices range over the set $\mathbb{N}_{n}$, unless we say otherwise. Thus we have

$$
\beta_{i}=\sum_{r} \beta_{r i}, \quad Q E_{i j}=\sum_{r} \beta_{r i} E_{r j}, \quad \text { and } \quad \varphi\left(Q E_{i j}\right)=\beta_{i} .
$$

Proposition 10.23. Let $n \in \mathbb{N}$ with $n \geq 2$, and let $P$ be an invertible sandwich matrix. Then each diagonal for $\left(\mathcal{M}^{o}(\mathbb{C}, P, n), \star_{P}\right)$ has the form

$$
\begin{aligned}
u= & \sum_{i, j, k, r, s} x_{j k} \beta_{r i} \beta_{s k} E_{r j} \otimes E_{s i} \\
& +\sum_{i, j, k, s} x_{j k}\left(1-\beta_{i}\right) \beta_{s k} \delta_{o} \otimes E_{s i}+\sum_{i, j, k, r} x_{j k}\left(1-\beta_{k}\right) \beta_{r i} E_{r j} \otimes \delta_{o} \\
& +\sum_{i, j, k} x_{j k}\left(1-\beta_{i}\right)\left(1-\beta_{k}\right) \delta_{o} \otimes \delta_{o}+\delta_{o} \otimes \delta_{o},
\end{aligned}
$$

where $\sum_{j} x_{j j}=1, \sum_{j} x_{j k}=0\left(k \in \mathbb{N}_{n}\right)$, and $\sum_{k} x_{j k}=0\left(j \in \mathbb{N}_{n}\right)$. Further, $\|u\|_{\pi} \geq 2 n+1$.

Proof. The form of the diagonal follows by combining equation (4.15) of Theorem 4.16 with the above lemma.

Let $A=\mathcal{M}^{o}(\mathbb{C}, P, n)$ and $B=\mathcal{M}(\mathbb{C}, P, n)$, and again identify $A$ with $B \oplus \mathbb{C} \delta_{o}$. 
Set $v=\sum_{i, j, k, r, s} x_{j k} \beta_{r i} \beta_{s k} E_{r j} \otimes E_{s i}$. We shall calculate the value of $\|v\|_{\pi}$ in $B \otimes B \subset A \otimes A$. In fact,

$$
\|v\|_{\pi}=\sum_{i, j, r, s}\left|\sum_{k} x_{j k} \beta_{r i} \beta_{s k}\right|=\sum_{i, r}\left|\beta_{r i}\right| \sum_{j, s}\left|\sum_{k} x_{j k} \beta_{s k}\right| .
$$

Each column of $Q$ contains terms with sum equal to 1 , and so

$$
\left|\beta_{i}\right| \geq \sum_{r}\left|\beta_{r i}\right| \geq 1 \quad\left(i \in \mathbb{N}_{n}\right)
$$

whence $\sum_{i, r}\left|\beta_{r i}\right| \geq n$. Now set $X=\left(x_{i j}\right) \in \mathbb{M}_{n}$, and note that

$$
X Q^{t}=\sum_{j, k, s} x_{j k} \beta_{s k} E_{k s}
$$

where $M^{t}$ is the transpose of a matrix $M$. The conditions imposed on $X$ imply that $X(1,1, \ldots, 1)^{t}=0$, and so the sum of each column of $X Q^{t}$ is 0 . We have $\operatorname{tr}\left(P^{t} X Q^{t}\right)=\operatorname{tr}(X)=1$, where 'tr' denotes the trace of a matrix. Let $\zeta_{j}=\left(P^{t} X Q^{t}\right)_{j j}\left(j \in \mathbb{N}_{n}\right)$. The $j^{\text {th. }}$ column of $X Q^{t}$ has some terms with sum $\zeta_{j}$, and the remaining terms must sum to $-\zeta_{j}$, and so the sum of the moduli of these terms is at least $2\left|\zeta_{j}\right|$. We have $\sum_{j} \zeta_{j}=1$, and so

$$
\sum_{j, s}\left|\sum_{k} x_{j k} \beta_{s k}\right| \geq 2 \sum_{j}\left|\zeta_{j}\right| \geq 2 .
$$

We conclude that $\|v\|_{\pi} \geq 2 n$.

The expression for $u$ contains the term $\delta_{o} \otimes \delta_{o}$, which is not in the linear span of the other terms in the expansion of $u$, and so we have $\|v\|_{\pi} \geq 2 n+1$, as required.

The following theorem now follows from our earlier estimates.

TheOREM 10.24. (i) Let $S=\mathcal{M}^{o}(G, n)$, where $G$ is an amenable group and $n \geq 2$. Then $A=\ell^{1}(S)=\mathcal{M}^{o}\left(\ell^{1}(G), n\right)$ has

$$
\left\|e_{A}\right\|_{1}=2 n-1, \quad C_{S}=2 n+1, \quad \text { and } \quad E_{S}=n+1 .
$$

(ii) Let $S=\mathcal{M}^{o}(G, P, n)$, where $G$ is an amenable group, $P$ is an invertible sandwich matrix, and $n \geq 2$. Then

$$
A=\ell^{1}(S)=\mathcal{M}^{o}\left(\ell^{1}(G), P, n\right)
$$

has

$$
\left\|e_{A}\right\|_{1} \geq 2 n-1, \quad C_{S} \geq 2 n+1, \quad \text { and } \quad n+1 \leq E_{S} \leq n^{2} .
$$


Proof. Clause (i) is immediate. We have verified clause (ii) in the special case where $G=\{e\}$; the lower bounds follow because there is a contractive epimorphism from $A$ onto $\mathcal{M}^{o}(\mathbb{C}, P, n)$.

We now examine the special case where $n=2$ and $A=\mathcal{M}^{o}(\mathbb{C}, P, 2)$ for an invertible matrix $P$. The above estimates give $\left\|e_{A}\right\|_{1} \geq 3$ and $C_{S} \geq 5$. First we see that a surprising uniqueness result holds in this case.

Proposition 10.25. Let $S=\mathcal{M}^{\circ}(2)$. Then the Banach algebra $A=\ell^{1}(S)=\mathcal{M}^{o}(\mathbb{C}, 2)$ has a unique diagonal

$$
\frac{1}{2}\left(E_{11}-E_{12}\right) \otimes\left(E_{11}-E_{21}\right)+\frac{1}{2}\left(E_{22}-E_{21}\right) \otimes\left(E_{22}-E_{12}\right)+\delta_{o} \otimes \delta_{o} .
$$

Further, $\left\|e_{A}\right\|_{1}=E_{S}=3$ and $C_{S}=5$.

Proof. The unique $2 \times 2$-matrix $\left(x_{i j}\right)$ satisfying the conditions that $x_{11}+x_{22}=1$ and that

$$
x_{11}+x_{12}=x_{21}+x_{22}=x_{11}+x_{21}=x_{12}+x_{22}=0
$$

is given by $x_{11}=x_{22}=1 / 2$ and $x_{12}=x_{21}=-1 / 2$. Thus the form of the unique diagonal, say $u$, is as specified. Clearly $\|u\|_{\pi}=5$, and so $C_{S}=5$.

The idempotents of $S$ are $e_{11}, e_{22}$, and $\delta_{o}$, and so $E_{S}=3$.

As noted in Example 4.14, there are just two sandwich matrices $P$ with $n=2$ for which $A=\mathcal{M}^{o}(\mathbb{C}, P, 2)$ is amenable; these are the identity matrix and the one that is specified by

$$
P=\left(\begin{array}{ll}
1 & 0 \\
1 & 1
\end{array}\right), \quad Q=P^{-1}=\left(\begin{array}{rr}
1 & 0 \\
-1 & 1
\end{array}\right)
$$

where we again identify $P$ and $Q$ with matrices in $\mathbb{M}_{2}$.

Proposition 10.26. Let $P$ be as above. Then the Banach algebra $A=\mathcal{M}^{\circ}(\mathbb{C}, P, 2)$ has a unique diagonal. Further,

$$
\left\|e_{A}\right\|_{1}=3, \quad E_{S}=4, \quad \text { and } \quad C_{S}=11 .
$$

Proof. Since $\varphi(Q)=1$, the identity of $A$ is $Q$, and so $\left\|e_{A}\right\|_{1}=3$.

Set $F_{i j}=Q E_{i j}\left(i, j \in \mathbb{N}_{2}\right)$, so that

$$
\begin{gathered}
F_{11}-F_{12}=\left(\begin{array}{rr}
1 & -1 \\
-1 & 1
\end{array}\right), \quad F_{11}-F_{21}=\left(\begin{array}{rr}
1 & 0 \\
-2 & 0
\end{array}\right), \\
F_{22}-F_{21}=\left(\begin{array}{rr}
0 & 0 \\
-1 & 1
\end{array}\right), \quad F_{22}-F_{12}=\left(\begin{array}{rr}
0 & -1 \\
0 & 2
\end{array}\right) .
\end{gathered}
$$


Also, $\varphi(A-Q A)$ takes the values $0,1,0$, and -1 , respectively, when $A=E_{11}-E_{12}, A=E_{11}-E_{21}, A=E_{22}-E_{21}$, and $A=E_{22}-E_{12}$, respectively. Thus it follows from Proposition 10.25 and Theorem 4.16 that the unique diagonal for $A$ is

$$
\begin{gathered}
\frac{1}{2}\left(F_{11}-F_{12}\right) \otimes\left(F_{11}-F_{21}\right)+\frac{1}{2}\left(F_{22}-F_{21}\right) \otimes\left(F_{22}-F_{12}\right) \\
+\frac{1}{2}\left(F_{11}-F_{12}\right) \otimes \delta_{o}-\frac{1}{2}\left(F_{22}-F_{21}\right) \otimes \delta_{o}+\delta_{o} \otimes \delta_{o} \\
=\left(E_{11} \otimes E_{11}-2 E_{11} \otimes E_{21}-E_{12} \otimes E_{11}+2 E_{12} \otimes E_{21}-E_{21} \otimes E_{11}\right. \\
+2 E_{21} \otimes E_{21}+E_{22} \otimes E_{11}-2 E_{22} \otimes E_{11}+E_{21} \otimes E_{12} \\
\left.-2 E_{21} \otimes E_{22}-E_{22} \otimes E_{21}+2 E_{22} \otimes E_{22}\right) / 2 \\
+\frac{1}{2}\left(\begin{array}{rr}
1 & -1 \\
0 & 0
\end{array}\right) \otimes \delta_{o}+\delta_{o} \otimes \delta_{o} .
\end{gathered}
$$

It follows that $C_{S}=(18 / 2)+2=11$. Clearly $E_{S}=4$.

In the case where $n \geq 3$, there are many matrices satisfying the conditions of Proposition 10.23, so certainly there is no uniqueness result, and hence it is hard to calculate amenability constants explicitly.

THEOREM 10.27. Let $S$ be a semigroup such that $\ell^{1}(S)$ is $C$-amenable for some $C<5$. Then $S$ is an amenable group.

Proof. First suppose that $S$ is a Rees matrix semigroup with zero of the form $\mathcal{M}^{o}(G, P, n)$, as in Theorem 10.20. In the case where $n=1$, so that $S=G^{o}$, we have $C_{S} \geq 5$ by Proposition 10.18. In general, by Theorem 10.24(ii), $C_{S} \geq 2 n+1$, and so $C_{S} \geq 5$ for $n \geq 2$. (In the case where $n=2$, we have $C_{S}=5$ or $C_{S}=11$ by the above two calculations.)

Now suppose that $S$ is a semigroup such that $\ell^{1}(S)$ is $C$-amenable for some $C<5$. By Theorem 10.12, $S$ has a principal series

$$
S=I_{0} \supsetneq I_{1} \supsetneq \cdots \supsetneq I_{m}=K(S)
$$

for which each quotient $I_{j-1} / I_{j}$ is a completely o-simple, regular semigroup with finitely many idempotents. By Corollary 2.6, $\ell^{1}\left(S / I_{1}\right)$ is $C$-amenable.

Assume towards a contradiction that $m \geq 1$. Then $S / I_{1}$ is a completely $o$-simple, regular semigroup with finitely many idempotents; each such semigroup has the form $\mathcal{M}^{o}(G, P, n)$ by Theorem 3.13. By our preliminary remark, the amenability constant of $\ell^{1}\left(S / I_{1}\right)$ is at least 5. Since there is contractive epimorphism from $\ell^{1}(S)$ onto $\ell^{1}\left(S / I_{1}\right)$, the amenability constant of $\ell^{1}(S)$ is at least 5 , a contradiction. Thus 
$m=0$, and so $K(S)=S$. By Corollary 10.6(ii), $K(S)$ is an amenable group.

Corollary 10.28. Let $S$ be a semigroup. Then the following are equivalent:

(a) $S$ is an amenable group;

(b) $\ell^{1}(S)$ is amenable with $A M\left(\ell^{1}(S)\right)=1$;

(c) $\ell^{1}(S)$ is amenable with $A M\left(\ell^{1}(S)\right)<5$.

In particular the interval $(1,5)$ is a set of 'forbidden values' for $C_{S}$.

EXAMPLE 10.29. We consider the special case of Theorem 10.20 in which $G$ is a singleton and $P$ is $P_{n}$ or $Q_{n}$, as defined in Example 3.43, so that our semigroup is $\mathbf{P}_{n}$ or $\mathbf{Q}_{n}$. The formulae for $P_{n}^{-1}$ and $Q_{n}^{-1}$ in $\mathbb{M}_{n}$ are exhibited in Example 4.15. For each $i \in \mathbb{N}_{n}$, we have

$$
\sum_{r=1}^{n}\left\|\left(P_{n}^{-1}\right)_{r i}\right\| \leq 2,
$$

and so $v\left(P_{n}\right)=2$. By Theorem 10.20, $C_{\mathbf{P}_{n}} \leq 16 n+1$. However, by equation (3.8), we have $E_{\mathbf{P}_{n}}=\left(n^{2}+n+2\right) / 2$, and so

$$
\frac{C_{\mathbf{P}_{n}}}{E_{\mathbf{P}_{n}}} \leq \frac{32 n+2}{n^{2}+n+2} \rightarrow 0 \quad \text { as } \quad n \rightarrow \infty .
$$

On the other hand,

$$
\frac{C_{\mathbf{Q}_{n}}}{E_{\mathbf{Q}_{n}}} \geq \frac{\left\|e_{\ell^{1}\left(\mathbf{Q}_{n}\right)}\right\|_{1}}{\left|E\left(\mathbf{Q}_{n}\right)\right|} \geq \frac{\left\|Q_{n}^{-1}\right\|}{\left|E\left(\mathbf{Q}_{n}\right)\right|} \rightarrow \infty \quad \text { as } \quad n \rightarrow \infty
$$

by (4.13). In particular, it is not true that $C_{S} / E_{S}$ is bounded either above or below, as had been conjectured.

Let $S$ be a semigroup such that $A:=\ell^{1}(S)$ is amenable. By Theorem 10.24(i), we can have $C_{S}=5,7,9, \ldots$, and we can also have $C_{S}=1$. We wonder which further values of $C_{S}$ can be attained. We have shown that numbers in the range $(1,5)$ are forbidden values for $C_{S}$, and we suspect that this is also true for the intervals $(5,7)$ and $(7,9)$.

[Added in proof. In [56], the authors obtain the following results, which partially answer some points in the above paragraph.

(1) Let $S$ be a finite, abelian semigroup with $E(S)=S$, so that $S$ is a semilattice. Then $C_{S}$ is always of the form $4 n+1$ for some $n \in \mathbb{Z}^{+}$, and each such value can be attained. 
(2) There is a finite, abelian semigroup $S$ (in fact, a Clifford semigroup) such that $C_{S}=43$, which is not of the form $4 n+1$ for any $n \in \mathbb{Z}^{+}$, and also there are Clifford semigroups $S$ such that $C_{S}$ is not an integer.

(3) There is no abelian semigroup $S$ such that $5<C_{S}<9$.] 



\section{CHAPTER 11}

\section{Amenability and weak amenability for certain Banach algebras}

In this chapter, we shall show that some algebras on compactifications of semigroups are not amenable and that some are not weakly amenable. In particular, we shall prove the following results. First, let $S$ be an infinite semigroup. Then $\ell^{1}(S)^{\prime \prime}=M(\beta S)$ and $\ell^{1}(\beta S)$ are not amenable. Second, let $G$ be an infinite locally compact group. Then $L^{1}(G)^{\prime \prime}$ is not weakly amenable; this latter result answers a question first raised in [54] and discussed in [23].

Amenability of $M(\beta S)$ Let $S$ be a semigroup. We shall first discuss the known results on the amenability of $M(\beta S)$; throughout, $M(\beta S)$ has the product $\square$.

The first result is essentially [54, Corollary 1.9]. Suppose that $S$ is an infinite semigroup, and assume that $M(\beta S)$ is amenable. Then, by Proposition $2.22, \ell^{1}(S)$ is also amenable; by Proposition 10.5(ii), it follows that $S$ is amenable. Thus, in the case where $S$ is either left or right cancellative, it follows from Proposition 10.5(iii) that $S$ is an amenable group, and this is shown to be a contradiction in Lemma 11.6, below. It is claimed in [54, Theorem 1.3] (see also [46, Theorem 4.9.3]) that $M(\beta S)$ is not amenable whenever $S$ is a weakly cancellative semigroup. But the proof given is not correct: it relies on [54, Theorem 1.2], and our Example 7.34 shows that this latter result fails even for an abelian, countable, weakly cancellative semigroup $S$. (However in this case $M(\beta S)$ is clearly not amenable.) The proof of $[\mathbf{5 4}$, Theorem 1.3] does apply whenever $S$ is an infinite semigroup which is weakly cancellative and nearly right cancellative. Indeed, suppose that $S$ is a weakly cancellative and nearly right cancellative semigroup, and assume that $M(\beta S)$ is amenable. Then $\ell^{1}(S)$ is not left strongly Arens regular by Proposition 2.26. However, by Theorem 12.15, to be proved below, $\ell^{1}(S)$ is left strongly Arens regular, the required contradiction.

Our first result on the amenability of $M(\beta S)$ and of $\ell^{1}(\beta S)$ does recover [54, Theorem 1.3] by a short argument; however stronger results will be proved later. 
Proposition 11.1. Let $S$ be an infinite, weakly left cancellative semigroup. Then neither $(M(\beta S), \square)$ nor $\left(\ell^{1}(\beta S), \square\right)$ is amenable.

Proof. Set $I=M\left(S^{*}\right)$. By Proposition 7.6, $I$ is a closed left ideal in $M(\beta S)$; certainly $I$ is complemented as a Banach space.

Assume towards a contradiction that $M(\beta S)$ is amenable. By Proposition 2.2(viii), I has a bounded approximate identity. But this contradicts Theorem 7.19.

Assume towards a contradiction that $\ell^{1}(\beta S)$ is amenable. Then, by Proposition $10.5(i i)$, the semigroup $(\beta S, \square)$ is amenable. But this contradicts Theorem 9.10(i).

EXAMPLE 11.2. Let $S$ be the right zero semigroup

on $S$; the product in $M(\beta S)$ is specified in Example 7.30. By Proposition 2.13 , both $M(\beta S)$ and $\ell^{1}(\beta S)$ are weakly amenable, but neither is amenable.

ExAmple 11.3. Set $S=\mathbb{N}_{\vee}$. It follows from Proposition 2.13 that both $M\left(S^{*}\right)$ and $\ell^{1}\left(S^{*}\right)$ are weakly amenable, but neither is amenable. It then follows from Proposition 2.2 that $M(\beta S)$ and $\ell^{1}(\beta S)$ are weakly amenable, but not amenable.

EXAmple 11.4. Let $S$ be the semigroup $\mathbb{N}_{\wedge}$. Assume towards a contradiction that $M(\beta S)$ is amenable. Then $\ell^{1}(S)$ is also amenable by Proposition 2.22, a contradiction of a remark in Example 10.10. Thus $M(\beta S)$ is not amenable.

By Proposition 2.13, $M\left(S^{*}\right)$ is weakly amenable; as in Example $10.10, \ell^{1}(S)$ is weakly amenable. However, as in Example 7.33, we have $M(\beta S)=M\left(S^{*}\right) \ltimes \ell^{1}(S)$, and so, by Proposition 2.2(iv), $M(\beta S)$ is weakly amenable.

The first step of our main proof is the following easy remark.

Proposition 11.5. Let $S$ be a semigroup such that $(M(\beta S), \square)$ is amenable, and let $I$ be an ideal in $S$. Then both $(M(\beta I), \square)$ and $(M(\beta(S / I)), \square)$ are amenable.

Proof. By Proposition 6.14(ii), $\beta I$ is a closed ideal in $\beta S$.

By Proposition 7.5(iii), $M(\beta I)$ is a closed ideal in $M(\beta S)$. Certainly $M(\beta I)$ is complemented in $M(\beta S)$ as a Banach space, and so it follows from Proposition 2.2(vii) that $M(\beta I)$ is amenable.

By Proposition 7.5(iv), $M(\beta(S / I))$ is a quotient of $M(\beta S)$ by a closed ideal, and so it follows from Proposition 2.2(vii) that $M(\beta(S / I))$ is amenable. 
Part of the following proof follows the argument of [51, Theorem 3.3]; we thank Fereidoun Ghahramani for pointing this out to us.

LEMMA 11.6. Let $S$ be a semigroup such that $(M(\beta S), \square)$ is amenable. Then $\ell^{1}(S)$ is amenable, $S$ is amenable, $S$ has a finite group ideal, $E(S)$ is finite, and each ideal in $S$ is a regular semigroup. Further, $(\beta S, \square)$ is amenable.

Proof. By Proposition 2.22, $\ell^{1}(S)$ is amenable. By Proposition 10.5(ii), $S$ is amenable, $E(S)$ is finite, and each ideal in $S$ is regular.

There exists $\mu_{0} \in \mathfrak{L}(S) ; \mu_{0}$ is an idempotent in $(M(\beta S), \square)$. Define $I=\mu_{0} \square M(\beta S)$. Then $I$ is a closed, complemented ideal in $M(\beta S)$, and so, by Proposition 2.2(vii), I has a bounded approximate identity, say $\left(\mu_{\alpha}\right)$. Let $\nu \in \mathfrak{L}(S)$. Then $\nu=\mu_{0} \square \nu \in I$, and so $\lim _{\alpha} \nu \square \mu_{\alpha}=\nu$. But also $\nu \square \mu_{\alpha}=\mu_{\alpha}$ for each $\alpha$, and so $\nu=\lim _{\alpha} \mu_{\alpha}$. This shows that $\nu=\mu_{0}$, and so $|\mathfrak{L}(S)|=1$.

It follows from Proposition 9.3 that $S$ has a finite group ideal. It is now clear that $(\beta S, \square)$ is amenable.

Lemma 11.7. Assume that there is an infinite semigroup $S$ such that $(M(\beta S), \square)$ is amenable. Then there is an infinite, completely osimple, regular semigroup $T$ with finitely many idempotents for which $(M(\beta T), \square)$ is amenable.

Proof. Let $F$ be the finite group ideal in $S$ specified in Lemma 11.6, and set $T=S / F$. Then $T$ is a semigroup with a zero. By Proposition 11.5, $M(\beta T)$ is amenable. Thus we may suppose that $S$ has a zero $o$.

Since $S$ is a regular semigroup and $E(S)$ is finite, it follows from Theorem 3.12 and Proposition 11.5 that we may suppose that there is an infinite, completely $O$-simple, regular semigroup $T$ with finitely many idempotents for which $(M(\beta T), \square)$ is amenable.

THEOREM 11.8. Let $S$ be a semigroup such that $(M(\beta S), \square)$ is an amenable Banach algebra. Then $S$ is finite.

Proof. Assume towards a contradiction that there is an infinite semigroup $S$ such that $M(\beta S)$ is amenable. By Lemma 11.7, we may suppose that $S$ is an infinite, completely o-simple, regular semigroup with finitely many idempotents.

By Proposition 3.13, $S$ is isomorphic to a regular Rees matrix semigroup with zero of the form $\mathcal{M}^{o}(G, P, m, n)$, where $G$ is an infinite group, and $m, n \in \mathbb{N}$. By Proposition $2.22, \ell^{1}(S)$ is amenable, and so 
we can apply the results in Example 10.11 to see that $m=n$, that $P$ is invertible in $\mathbb{M}_{n}\left(\ell^{1}(G)\right)$, and that $\ell^{1}(S) / \mathbb{C} \delta_{o}$ is topologically isomorphic to $\mathbb{M}_{n}\left(\ell^{1}(G)\right)$. Hence $M(\beta S) / \mathbb{C} \delta_{o}$ is topologically isomorphic to $\mathbb{M}_{n}(M(\beta G))$, which is thus amenable. By Theorem 2.7(i), $M(\beta G)$ is amenable. But this contradicts both Proposition 11.1 and Lemma 11.6 .

This concludes the proof of the theorem.

Let $S$ be a semigroup, and let $X$ be an introverted

$C^{*}$-subalgebra of $\ell^{\infty}(S)$ (for example, let $X=W A P(S)$ ). Suppose that $X^{\prime}$ is an amenable Banach algebra. It would be of interest to determine conditions on $X$ that force $S$ to be finite.

Amenability of $\ell^{1}(\beta S)$ Now let us turn to the amenability of $\ell^{1}(\beta S)$.

THEOREM 11.9. Let $S$ be a semigroup such that $\left(\ell^{1}(\beta S), \square\right)$ is an amenable Banach algebra. Then $S$ is finite.

Proof. Assume towards a contradiction that $S$ is an infinite semigroup such that $\left(\ell^{1}(\beta S), \square\right)$ is an amenable Banach algebra.

By Proposition 10.5(ii), $\beta S$ is a regular semigroup and $E(\beta S)$ is finite. It follows from Proposition 6.23(i) that each subgroup of $S$ is finite.

By Proposition 6.6, the semigroup $S$ itself is regular, and of course $E(S)$ is finite. Thus, by Theorem 3.12, $S$ contains an infinite subgroup.

We have obtained the required contradiction.

Weak amenability of $M\left(S^{*}\right)$ Let $S$ be a semigroup. Before considering the weak amenability of the Banach algebra $M(\beta S)$, we look at the weak amenability of $M\left(S^{*}\right)$. We recall that Corollary 7.9 shows that $M\left(S^{*}\right)$ is a Banach algebra whenever $S^{*}$ is a subsemigroup of $\beta S$.

Proposition 11.10. Let $S$ be a semigroup such that $S^{*}$ is a subsemigroup of $\beta S$ with $\overline{S_{[2]}^{*}} \neq S^{*}$. Then $M\left(S^{*}\right)$ is not weakly amenable.

Proof. By Corollary 7.9, $\overline{M\left(S^{*}\right)^{2}} \subset M\left(\overline{S_{[2]}^{*}}\right)$. Since $\overline{S_{[2]}^{*}} \neq S^{*}$, $M\left(S^{*}\right)$ is not essential, and hence not weakly amenable. Then

Proposition 11.11. Let $S$ be an infinite, cancellative semigroup.

$M\left(S^{*}\right)$ is not weakly amenable.

Proof. By Corollary 7.8, $M\left(S^{*}\right)$ is not essential, and hence it is not weakly amenable. 
Weak amenability of $M(\beta S)$ We now consider when $M(\beta S)$ itself is weakly amenable. We first give an easy example, not covered by later results, where it is not weakly amenable.

EXAMPLE 11.12. Let $S$ be an infinite trivial semigroup

at $p \in S$, so that $s t=p(s, t \in S)$. Thus $S$ is not weakly cancellative. We note that

$$
\mu \square \nu=\langle\mu, 1\rangle\langle\nu, 1\rangle p \quad(\mu, \nu \in M(\beta S)),
$$

and so $M(\beta S)^{[2]}=\mathbb{C} p$ and $M(\beta S)$ is not essential. By Proposition $2.2($ iii),$M(\beta S)$ is not weakly amenable.

The following result is immediate from equation (7.4) and Proposition $2.2(\mathrm{x})$.

Proposition 11.13. Let $S$ be a weakly cancellative semigroup. Suppose that $(M(\beta S), \square)$ is weakly amenable. Then $\ell^{1}(S)$ is weakly amenable.

We do not know of a semigroup $S$ such that $M(\beta S)$ is weakly amenable, but $\ell^{1}(S)$ is not weakly amenable.

We now approach our main results on weak amenability. In the following, we again often write $u v$ for $u \square v$ when $u, v \in \beta S$.

THEOREM 11.14. Let $S$ be an infinite, weakly cancellative semigroup. Assume that there is a non-empty subset $V$ of $S^{*}$ such that

$$
s^{-1} V t^{-1}=V \quad\left(s, t \in S^{\#}\right)
$$

and

$$
(\mu \square \nu)(\{v\})=0 \quad\left(v \in V, \mu, \nu \in M\left(S^{*}\right)\right) .
$$

Then there is a non-zero, continuous point derivation

at the discrete augmentation character of the Banach algebra $M(\beta S)$, and $M(\beta S)$ is not weakly amenable.

Proof. The discrete augmentation character on $S$ is denoted by $\widetilde{\varphi}_{S}$. Set $A=\ell^{1}(S)$, so that $A^{\prime \prime}=M(\beta S)$.

For each $\mu \in A^{\prime \prime}$, define

$$
\Lambda(\mu)=\sum\{\mu(\{v\}): v \in V\} .
$$

Then $|\Lambda(\mu)| \leq\|\mu\|$. Clearly $\Lambda$ is a linear functional on $A^{\prime \prime}$, and so the map $\Lambda: \mu \mapsto \Lambda(\mu)$ is a continuous linear functional on $A^{\prime \prime}$. Since $V \neq \emptyset$, we have $\Lambda \neq 0$. We have $\Lambda \mid A=0$ because $V \subset S^{*}$; further, it follows from the hypothesis (11.2) that

$$
\Lambda(\mu \square \nu)=0 \quad\left(\mu, \nu \in M\left(S^{*}\right)\right) .
$$


Let $s \in S$ and $\mu \in A^{\prime \prime}$. Then

$$
\begin{aligned}
\Lambda(s \square \mu) & =\sum\{(s \square \mu)(\{v\}): v \in V\} \\
& =\sum\left\{\mu\left(\left\{s^{-1} v\right\}\right): v \in V\right\} \\
& =\sum\left\{\mu(\{u\}): u \in s^{-1} V\right\}=\Lambda(\mu)
\end{aligned}
$$

because $s^{-1} V=V$. Similarly, $\Lambda(\mu \square s)=\Lambda(\mu)$ because $V s^{-1}=V$. Thus

$$
\Lambda(f \square \mu)=\Lambda(\mu \square f)=\varphi_{S}(f) \Lambda(\mu) \quad\left(f \in A, \mu \in M\left(S^{*}\right)\right) .
$$

We claim that the continuous linear functional $\Lambda$ is a point derivation

at $\widetilde{\varphi}_{S}$. By (7.4), $M(\beta S)=A \ltimes M\left(S^{*}\right)$. Take $a, b \in A^{\prime \prime}$, say $a=$ $(f, \mu)$ and $b=(g, \nu)$, where $f, g \in A$ and $\mu, \nu \in M\left(S^{*}\right)$. Then

$$
\begin{aligned}
\Lambda(a \square b) & =\Lambda(f \star g)+\Lambda(f \square \nu)+\Lambda(\mu \square g)+\Lambda(\mu \square \nu) \\
& =\Lambda(f \square \nu)+\Lambda(\mu \square g) \\
& =\varphi_{S}(f) \Lambda(\nu)+\varphi_{S}(g) \Lambda(\mu) \\
& =\widetilde{\varphi}_{S}(a) \Lambda(b)+\widetilde{\varphi}_{S}(b) \Lambda(a) .
\end{aligned}
$$

Hence $\Lambda$ is indeed a point derivation at $\widetilde{\varphi}_{S}$.

THEOREM 11.15. Let $S$ be an infinite, cancellative semigroup.

Then there is a non-zero, continuous point derivation at the discrete augmentation character of the Banach algebra $M(\beta S)$, and $M(\beta S)$ is not weakly amenable.

Proof. By Theorem 7.13, there is a non-empty subset $V$ of $S^{*}$ such that $s^{-1} V t^{-1}=V(s, t \in S)$ and such that equation (11.2) holds. Thus the result follows from the theorem.

EXAMPLE 11.16. Let $S=\mathbb{Z}^{2}$ be the semigroup specified in Example 3.32. It follows from Theorem 11.1 that $M(\beta S)$ is not amenable. There is an epimorphism $\theta: S \rightarrow \mathbb{Z}$. By Theorem 11.15, there is a non-zero, continuous point derivation on $M(\beta \mathbb{Z})$, and so $M(\beta S)$ is not weakly amenable.

We noted in Example 4.8 that $\ell^{1}(S)$ is not necessarily weakly amenable when $S$ is a finite semigroup, and we noted in Example 11.3 that there is an infinite, abelian, weakly cancellative semigroup $S$ such that $M(\beta S)$ is weakly amenable, so that the above theorem would be false if we replaced 'cancellative' by 'weakly cancellative' in the statement. 
Locally compact groups We now apply our results to resolve an open question about group algebras of locally compact groups.

TheOREM 11.17. Let $G$ be a locally compact group. Then the following conditions on $G$ are equivalent:

(a) the group $G$ is infinite;

(b) there is a non-zero, continuous point derivation at the discrete augmentation character of $\left(L^{1}(G)^{\prime \prime}, \square\right)$;

(c) the Banach algebra $\left(L^{1}(G)^{\prime \prime}, \square\right)$ is not weakly amenable.

Proof. (a) $\Rightarrow$ (b) We first suppose that $G$ is not discrete. By Theorem 10.4(iii), there is a non-zero, continuous point derivation at the discrete augmentation character on $M(G)$. But now, by Proposition 8.9 (with $X=L^{\infty}(G)$ ), there is a non-zero, continuous point derivation at the discrete augmentation character of $\left(L^{1}(G)^{\prime \prime}, \square\right)$.

We next suppose that the group $G$ is discrete (and infinite), so that $L^{1}(G)=\ell^{1}(G)$ and $\left(L^{1}(G)^{\prime \prime}, \square\right)=(M(\beta G), \square)$. Now $G$ satisfies all the conditions imposed on $S$ in Theorem 11.15, and so the result follows from that theorem.

(b) $\Rightarrow$ (c) This follows from Proposition 2.2(iii).

(c) $\Rightarrow$ (a) Assume that the group $G$ is finite. Then $G$ is amenable, and so $\ell^{1}(G)=\left(L^{1}(G)^{\prime \prime}, \square\right)$ is an amenable Banach algebra, and hence $\left(L^{1}(G)^{\prime \prime}, \square\right)$ is weakly amenable, a contradiction of (c).

The fact that $\left(L^{1}(G)^{\prime \prime}, \square\right)$ is not weakly amenable in the case where $G$ is an infinite abelian group was first shown by Forrest in [47] by a different argument. Further partial results are given in [96]. The arguments of $[\mathbf{2 3}, \S 4]$ show the same result in the case where $G$ is a maximally almost periodic group, but do not extend to general infinite groups.

We now extend the above result to cover more general algebras.

THEOREM 11.18. Let $G$ be an infinite locally compact group, and let $X$ be an introverted $C^{*}$-subalgebra

of $L^{\infty}(G)$ with $W A P(G) \subset X$. Then there is a non-zero, continuous point derivation at the discrete augmentation character of $\left(X^{\prime}, \square\right)$, and the Banach algebra $\left(X^{\prime}, \square\right)$ is not weakly amenable.

Proof. As in the above proof, we may reduce to the case where $G$ is discrete.

Suppose that $G$ is discrete. By Proposition 8.5, there is a non-empty subset $V$ of $\Phi_{X}$ such that $s V t=V(s, t \in G)$ and $V \cap\left(\Phi_{X}^{*}\right)_{[2]}=\emptyset$. 
Define a continuous linear functional $\Lambda$ on $M\left(\Phi_{X}\right)$ by setting

$$
\Lambda(\mu)=\sum\{\mu(v): v \in V\} \quad\left(\mu \in M\left(\Phi_{X}\right) .\right.
$$

As in Theorem 11.14, $\Lambda$ is the required continuous, non-zero point derivation. 


\section{CHAPTER 12}

\section{Topological centres}

In this chapter, we shall investigate the topological centres

of some Banach algebras and some semigroups. We shall discover some small sets that are determining for the left topological centre, in the sense of Definition 12.4, below.

Arens regularity of semigroup algebras We first record the following condition for the Arens regularity of a semigroup algebra $\ell^{1}(S)$; the result was first proved in [137] and follows easily from Theorem 2.28. Explicit conditions for the Arens regularity of $\ell^{1}(S)$ are given in [138]; a more extended version of the following theorem is given as $[\mathbf{4}$, Theorem (2.7)]. Results in [16] and [21] discuss the Arens regularity of weighted convolution algebras of the form $\ell^{1}(G, \omega)$.

THEOREM 12.1. The following are equivalent for a semigroup $S$;

(a) $\ell^{1}(S)$ is

Arens regular;

(b) $W A P(S)=\ell^{\infty}(S)$;

(c) there do not exist sequences $\left(s_{m}\right)$ and $\left(t_{n}\right)$ in $S$ such that the two sets $\left\{s_{m} t_{n}: m<n\right\}$ and $\left\{s_{m} t_{n}: m>n\right\}$ are disjoint.

For example, let $S$ be a right zero semigroup. Then $\ell^{1}(S)$ is Arens regular, as explained in Example 7.30. The Arens regularity of various semigroup algebras has also been studied by Esslamzadeh; the following result, among others, is proved in [38].

THEOREM 12.2. Let $S$ be an infinite, regular semigroup with finitely many idempotents. Then $\ell^{1}(S)$ is not Arens regular.

An example within Example 7.14 exhibits an infinite, regular semigroup $S$ with $E(S)$ finite for which $\ell^{1}(S)$ is not strongly Arens irregular.

Sets determining for the topological centre We now introduce a new condition related to the topological centre of a Banach algebra.

Definition 12.3. Let $A$ be a Banach algebra. A subset $V$ of $A^{\prime \prime}$ is determining for the left topological centre

of $A^{\prime \prime}$ if $\Phi \in A$ whenever $\Phi \in A^{\prime \prime}$ and $\Phi \square \Psi=\Phi \diamond \Psi(\Psi \in V)$. 
Clearly, if there is such a set $V$, then $A$ is left strongly Arens irregular. We note that, for $\Phi, \Psi \in A^{\prime \prime}$, we have $\Phi \square \Psi=\Phi \diamond \Psi$ if and only if $\lim _{\alpha} \Phi \cdot a_{\alpha}=\Phi \square \Psi$ whenever $\left(a_{\alpha}\right)$ is a net in $A$ with $\lim _{\alpha} a_{\alpha}=\Psi$ in $\left(A^{\prime \prime}, \sigma\left(A^{\prime \prime}, A^{\prime}\right)\right)$. The condition that $L_{\Phi}$, defined on $\left(A^{\prime \prime}, \square\right)$, is continuous at $\Psi$ is a stronger condition

Definition 12.4. Let $S$ be an infinite semigroup. A subset $V$ of $S^{*}$ is determining for the left topological centre

of $M(\beta S)$ if the only element $\mu$ of $M\left(S^{*}\right)$ such that $\mu \square v=\mu \diamond v$ for each $v \in V$ is $\mu=0$. A subset $V$ of $S^{*}$ is determining for the left topological centre of $\beta S$ if there are no elements $u$ of $S^{*}$ such that $u \square v=u \diamond v$ for each $v \in V$.

We note that the set $V$ is determining for the left topological centre of $M(\beta S)$ if the only element $\mu$ of $M_{\mathbb{R}}\left(S^{*}\right)$ such that $\mu \square v=\mu \diamond v$ for each $v \in V$ is $\mu=0$. Suppose that $S^{*}$ is determining for the left topological centre of $M(\beta S)$ and of $\beta S$, respectively. Then $\ell^{1}(S)$ and $S$, respectively, are left strongly Arens irregular.

We note that $V$ is determining for the left topological centre of $M(\beta S)$ if and only if, for each $v \in V$, we have $\lim _{s \rightarrow v} \mu \cdot s=\mu \square v$ whenever $s \rightarrow v$ with $s \in S$; again this is weaker than the condition that the map $u \mapsto \mu \square u, \beta S \rightarrow M(\beta S)$, is continuous at each point of $V$.

In the case where the semigroup $S$ is abelian, we use the phrase determining for the topological centre.

Our results will extend known results, which we first summarize.

The first result in this area seems to be one of Butcher $[\mathbf{1 4}$, Theorem 4.4.2], which states in a different language that $\ell^{1}(S)$ is strongly Arens irregular whenever $S$ is a countable, cancellative abelian semigroup such that $S^{*}$ is the union of two disjoint left ideals of $\beta S$; we shall recover this within Corollary 12.6, below. Various generalizations of these results for abelian semi-topological semigroups are given by Parsons in [115].

Second, the following theorem is proved in [92]. Let $S$ be a cancellative semigroup, and suppose that $t \in \beta S$ is such that $t \square \mu=t \diamond \mu$ for each $\mu \in M(\beta S)$. Then $t \in S$. (In [92], the result is claimed in the case where $S$ is only weakly cancellative, but the proof does not hold in this generality, as pointed out by Bami in [5]; we shall recover a stronger form of this claimed theorem for weakly cancellative semigroups in Theorem 12.20, below.) Our result extends the result proved in [92] because it only requires that the equation $t \square u=t \diamond u$ hold for each $u \in \beta S$; indeed, it only requires this equation to hold for each $u$ in a 'two-element' subset of $S^{*}$. 
Third, let $G$ be a group. Then it is proved in $[\mathbf{9 8}]$ by what is called in [118] a 'lengthy argument' that $G$ is strongly Arens irregular (and that the analogous result holds for all locally compact groups $G$ ). Thus the only element $\mu$ of $M\left(G^{*}\right)$ such that $\mu \square \nu=\mu \diamond \nu$ for each $\nu \in M\left(G^{*}\right)$ is $\mu=0$. It is proved by Neufang in [110] that $G^{*}$ is determining for the left topological centre of $M(\beta G)$. Theorem 12.15 , below, extends this to cancellative (and more general) semigroups. In fact Neufang's theorem also applies to various weighted convolution algebras; see also [20] and [21, Corollary 11.10].

Our results in this section will considerably strengthen all the above results, and will provide shorter proofs. We shall prove that, for many semigroups $S$, there is a two-element subset of $S^{*}$ that is determining for the left topological centre of $M(\beta S)$.

At least for abelian semigroups $S$, no singleton is determining for the topological centre because $x \square x=x \diamond x$ for each $x \in S^{*}$. We shall give an example concerning this at the end of the chapter.

New results We now give our new results.

THEOREM 12.5. Let $S$ be an infinite semigroup, and let $K$ be a non-empty, closed subset of $S^{*}$. Suppose that there exist subsets $U$ and $V$ of $S$ and right cancellable elements $a \in U^{*}$ and $b \in V^{*}$ such that the following conditions are satisfied:

(i) $U \cap V=\emptyset$;

(ii) $S \square a \subset \bar{U}$ and $S \square b \subset \bar{V}$;

(iii) for each $x \in K$, either $(x \diamond S) \cap \bar{U}=\emptyset$ or $(x \diamond S) \cap \bar{V}=\emptyset$.

Let $\mu \in M(\beta S)$ and $\nu \in M(K)$, and take $\varepsilon>0$. Then there exist two functions $\lambda_{a}, \lambda_{b} \in C(\beta S)_{[1]}$ such that

$$
\left\langle\mu \square a, \lambda_{a}\right\rangle>\|\mu\|-\varepsilon \quad \text { and }\left\langle\mu \square b, \lambda_{b}\right\rangle>\|\mu\|-\varepsilon
$$

and either

$$
\left|\left\langle\nu \diamond x, \lambda_{a}\right\rangle\right| \leq \frac{1}{2}\|\nu\| \quad(x \in \beta S)
$$

or

$$
\left|\left\langle\nu \diamond x, \lambda_{b}\right\rangle\right| \leq \frac{1}{2}\|\nu\| \quad(x \in \beta S) .
$$

Let $\mu \in M(K)$ with $\mu \square a=\mu \diamond a$ and $\mu \square b=\mu \diamond b$. Then $\mu=0$.

Proof. Let $\mu \in M(\beta S)$ and $\nu \in M(K)$, and take $\varepsilon>0$.

Since $a$ and $b$ are right cancellable in $\beta S$, it follows from Proposition 4.4(iii) that $\|\mu \square a\|=\|\mu \square b\|=\|\mu\|$. Thus there exist $\lambda_{a}, \lambda_{b} \in C(\beta S)_{[1]}$ such that equation (12.1) is satisfied. 
By (ii), $\beta S \square a \subset \bar{U}$ and $\beta S \square b \subset \bar{V}$, and so

$$
\operatorname{supp}(\mu \square a) \subset \bar{U} \quad \text { and } \quad \operatorname{supp}(\mu \square b) \subset \bar{V} .
$$

By (i), $\bar{U} \cap \bar{V}=\emptyset$. Thus, by replacing $\lambda_{a}$ by $\lambda_{a} \cdot \chi_{\bar{U}}$ and $\lambda_{b}$ by $\lambda_{b} \cdot \chi_{\bar{V}}$, we may suppose that $\lambda_{a}$ and $\lambda_{b}$ vanish outside $\bar{U}$ and $\bar{V}$, respectively.

Let $f=\sum_{i=1}^{m} \alpha_{i} x_{i}$, where $\alpha_{1}, \ldots, \alpha_{m} \in \mathbb{C}$ with $\sum_{i=1}^{m}\left|\alpha_{i}\right|=1$, and $x_{1}, \ldots, x_{m} \in K$. Take $i \in \mathbb{N}_{m}$. By (iii),

either $\left\langle x_{i} \diamond s, \lambda_{a}\right\rangle=0(s \in S) \quad$ or $\quad\left\langle x_{i} \diamond s, \lambda_{b}\right\rangle=0 \quad(s \in S)$.

Thus

$$
\text { either }\left|\left\langle f \diamond s, \lambda_{a}\right\rangle\right| \leq \frac{1}{2}(s \in S) \quad \text { or } \quad\left|\left\langle f \diamond s, \lambda_{b}\right\rangle\right| \leq \frac{1}{2}(s \in S) \text {, }
$$

and hence either

$$
\left|\left\langle\nu \diamond s, \lambda_{a}\right\rangle\right| \leq \frac{1}{2}\|\nu\| \quad(s \in S) \quad \text { or } \quad\left|\left\langle\nu \diamond s, \lambda_{b}\right\rangle\right| \leq \frac{1}{2}\|\nu\| \quad(s \in S) .
$$

Since the map $x \mapsto \nu \diamond x, \beta S \rightarrow M\left(S^{*}\right)$, is continuous, it follows that either equation (12.2) or (12.3) is satisfied.

Now let $\mu \in M(K)$ be such that $\mu \square a=\mu \diamond a$ and $\mu \square b=\mu \diamond b$, and assume towards a contradiction that $\mu \neq 0$; we may suppose that $\|\mu\|=1$. We apply the above remark with $\nu=\mu$ and $\varepsilon=1 / 2$. Since $\mu \square a=\mu \diamond a$ and $\mu \square b=\mu \diamond b$, this is indeed a contradiction.

We make two remarks on the above proof.

First, the fact that the elements $a$ and $b$ are right cancellable was used only to establish that $\|\mu \square a\|=\|\mu\|$ and that $\|\mu \square b\|=\|\mu\|$. However these two equalities hold for all elements $a, b \in S^{*}$ whenever $\mu \in M(\beta S)^{+}$(and in particular for $\mu \in \beta S$ ).

Second, in the last clause, we did not really need the condition that $\mu \square a=\mu \diamond a$ and $\mu \square b=\mu \diamond b$, but only the weaker condition that

$$
\max \{\|\mu \square a-\mu \diamond a\|,\|\mu \square b-\mu \diamond b\|\}<\frac{1}{2}\|\mu\|
$$

to obtain a contradiction. We do not know whether or not the number ' $1 / 2$ ' on the right-hand side of (12.4) can be replaced by ' 1 '. Note that we cannot be sure that there are (necessarily left-cancellable) elements $a$ of $S^{*}$ such that $\|a \square \nu\|=\|\nu\| \quad\left(\nu \in M\left(S^{*}\right)\right)$.

The following corollary subsumes the result of Butcher mentioned above (taking $S=\mathbb{Z}, U=\mathbb{N}$, and $V=-\mathbb{N}$ ). The result also covers the case of the free semigroup $S=\mathbb{S}_{2}$ and many other semigroups.

Corollary 12.6. Let $S$ be an infinite semigroup. Suppose that $U$ and $V$ are disjoint, infinite subsemigroups of $S$ such that $U^{*}$ and $V^{*}$ are left ideals in $S^{*}$. Then, for each pair $\{a, b\}$ of right cancellable 
elements with $a \in U^{*}$ and $b \in V^{*}$, the set $\{a, b\}$ is determining for the left topological centre of $M(\beta S)$.

Suppose, further, that $S$ is cancellative. Then such a pair $\{a, b\}$ of right cancellable elements exists, and so $\ell^{1}(S)$ is left strongly Arens irregular.

Proof. We apply Theorem 12.5 , taking $K=S^{*}$. It is clear that the conditions of Theorem 12.5 are satisfied, and so $\{a, b\}$ is determining for the left topological centre of $M(\beta S)$.

Suppose that $S$ is cancellative. Then it follows from Proposition 6.21 that there are such right cancellable elements $a \in U^{*}$ and $b \in V^{*}$. Hence $\ell^{1}(S)$ is left strongly Arens irregular.

Example 7.34 exhibits an infinite, abelian, weakly cancellative semigroup $S$ such that $\ell^{1}(S)$ is not strongly Arens irregular. Thus we cannot replace 'cancellative' by 'weakly cancellative' in the above corollary.

Countable semigroups We establish our main theorem for certain countable semigroups; for the uncountable case, see Theorem 12.15.

THEOREM 12.7. Let $S$ be an infinite, countable semigroup such that $S$ is weakly cancellative and nearly right cancellative. Then there exist $a$ and $b$ in $S^{*}$ that are right cancellable in $(\beta S, \square)$ and such that the two-element set $\{a, b\}$ is determining for the left topological centre of $M(\beta S)$.

Suppose that $S$ is also nearly left cancellative. Then $\ell^{1}(S)$ is strongly Arens irregular.

Proof. We may suppose that $S$ has an identity $e_{S}$. We enumerate $S$ as a sequence $\left(s_{n}\right)$, where $s_{0}=e_{S}$, and we use the notation involving $\preccurlyeq,[t]$, and $[F]$ from Chapter $3 ;[F]$ is finite whenever $F$ is finite.

Since $S$ is nearly right cancellative, there is an infinite subset $X$ of $S$ such that, for each $s, t \in S$ with $s \neq t$, the set

$$
E_{s, t}:=\{x \in X: s x=t x\}
$$

is finite. We may suppose that $e_{S} \in X$.

We shall construct a sequence $\left(t_{n}\right)$ in $X$ by induction. Set $t_{0}=s_{0}$. Once $t_{0}, \ldots, t_{n}$ have been defined, set

$$
T_{n}=\left\{s_{0}, \ldots, s_{n}, t_{1}, \ldots, t_{n}\right\} .
$$

The sequence will satisfy the following three conditions for each $n \in \mathbb{Z}^{+}$:

(i) $s T_{n} \cap\left[T_{n}\right]=\emptyset$ whenever $s \in S$ with $t_{n+1} \preccurlyeq s$;

(ii) $s t_{n+1} \neq t t_{n+1}$ whenever $s, t \in T_{n}$ with $s \neq t$;

(iii) $r s \prec t t_{n+1}$ whenever $r, s, t \in T_{n}$. 
Take $n \in \mathbb{Z}^{+}$, and assume that $t_{0}, \ldots, t_{n}$ have been specified in $X$. Since $S$ is weakly cancellative, the set $T_{n}^{-1}\left[T_{n}^{2}\right]$ is finite; since $S$ is nearly right cancellative, $\bigcup\left\{E_{s, t}: s, t \in T_{n}, s \neq t\right\}$ is finite. We choose $t_{n+1} \in X$ so that $t_{n+1}$ is strictly greater than the maximum of the union of these sets; the element $t_{n+1}$ is such that the above conditions (i)-(iii) are satisfied. The inductive construction continues.

Note that $s_{n} \preccurlyeq t_{n}\left(n \in \mathbb{Z}^{+}\right)$, and so $\bigcup\left\{\left[t_{n}\right]: n \in \mathbb{Z}^{+}\right\}=S$. Also $S^{*}=\left(S \backslash\left[t_{k}\right]\right)^{*}$ for each $k \in \mathbb{N}$.

Define $\varphi: S \rightarrow \mathbb{Z}^{+}$by setting

$$
\varphi(s)=\min \left\{n \in \mathbb{Z}^{+}: s \in\left[t_{n}\right]\right\} \quad(s \in S) .
$$

Suppose that $\varphi(s)=m \in \mathbb{N}$. It follows from (iii) that $t_{n-1} \prec s t_{n} \prec t_{n+1}$ whenever $n>m$, and so

$$
\varphi\left(s t_{n}\right) \in\{n, n+1\} \quad(n>m) .
$$

Now suppose that $m \geq 2$ and that $k \leq m-2$. Then $t_{m-2} \prec s s_{k}$ by (i) because $s T_{m-2} \cap\left[T_{m-2}\right]=\emptyset$. Further, $s s_{k} \prec t_{m+1}$ by (iii) because $s \in T_{m}$. Thus $\varphi\left(s s_{k}\right) \in\{m-1, m, m+1\}$, and so

$$
\varphi\left(s s_{k}\right) \in\{m-1, m, m+1\} \quad(m \geq k+2) .
$$

For $s \in S$, set $\gamma(s) \equiv \varphi(s)(\bmod 8)$. Then $\gamma: S \rightarrow \mathbb{Z}_{8}$ has a continuous extension, also denoted by $\gamma$, to a map $\gamma: \beta S \rightarrow \mathbb{Z}_{8}$. It follows from (12.6) that, for each $x \in S^{*}$ and $s \in S$, we have

$$
\gamma(x \square s)=\gamma(x \diamond s) \in\{\gamma(x)-1, \gamma(x), \gamma(x)+1\} \subset \mathbb{Z}_{8} .
$$

Set

$$
A=\left\{t_{n}: \gamma\left(t_{n}\right)=1\right\}, \quad B=\left\{t_{n}: \gamma\left(t_{n}\right)=5\right\},
$$

so that $A$ and $B$ are infinite subsets of $S$. Then set

$$
U=\{s \in S: \gamma(s) \in\{1,2\}\}, \quad V=\{s \in S: \gamma(s) \in\{5,6\}\},
$$

so that $A \subset U$ and $B \subset V$.

Choose $a \in A^{*}$ and $b \in B^{*}$. We claim that $a$ is right cancellable in $\beta S$. Indeed, let $u_{1}$ and $u_{2}$ be distinct points of $\beta S$, and take disjoint $N_{1}$ and $N_{2}$ in $S$ with $u_{j} \in \overline{N_{j}}(j=1,2)$. For $j=1,2$, set

$$
Y_{j}=\left\{s_{m} t_{n}: s_{m} \in N_{j}, t_{n} \in A, m<n\right\},
$$

so that $Y_{j} \in u_{j} \square a$. Take $m_{1}, m_{2}, n_{1}, n_{2} \in \mathbb{N}$ with $m_{1}<n_{1}, m_{2}<n_{2}$, and $m_{1} \neq m_{2}$. Then $s_{m_{1}} t_{n_{1}} \neq s_{m_{2}} t_{n_{2}}$ : this holds for $n_{1}<n_{2}$ by (iii) and for $n_{1}=n_{2}$ by (ii). It follows that $Y_{1} \cap Y_{2}=\emptyset$, and so $u_{1} \square a \neq u_{2} \square a$, as required. Similarly, $b$ is right cancellable in $\beta S$.

It follows from (12.5) that $S \square a \subset \bar{U}$ and $S \square b \subset \bar{V}$.

Let $x \in S^{*}$, set $k=\gamma(x)$, and take $s \in S$. Suppose that $x \diamond s \in \bar{U}$. Then it follows from (12.7) that $k \in\{0,1,2,3\}$. Suppose that $x \diamond s \in \bar{V}$. 
Then, similarly, $k \in\{4,5,6,7\}$. Thus either $(x \diamond S) \cap \bar{U}=\emptyset$ or $(x \diamond S) \cap \bar{V}=\emptyset$.

We have shown that the hypotheses of Theorem 12.5 are satisfied (with $K=S^{*}$ ), and so $\{a, b\}$ is determining for the left topological centre of $M(\beta S)$. Thus $\ell^{1}(S)$ is left strongly Arens irregular.

Now suppose that $S$ is also nearly left cancellative. Then $\ell^{1}\left(S^{\text {op }}\right)$ is left strongly Arens irregular, and so $\ell^{1}(S)$ is right strongly Arens irregular, and hence strongly Arens irregular.

There is a small modification of the above proof; it shows that, at least for cancellative semigroups, 'most' pairs $\{a, b\}$ are determining for the left topological centre.

TheOREM 12.8. Let $S$ be an infinite, countable, cancellative semigroup.

(i) Let $P$ and $Q$ be infinite subsets of $S$. Then there exists $a \in P^{*}$ and $b \in Q^{*}$ such that $a$ and $b$ are right cancellable in $(\beta S, \square)$ and such that the two-element set $\{a, b\}$ is determining for the left topological centre of $M(\beta S)$.

(ii) $(\mathrm{CH})$ We may suppose further that $a$ and $b$ are $P$-points in $S^{*}$.

Proof. (i) We carry through the above proof, without mention of the set $X$, but we choose the sequence $\left(t_{n}\right)$ to have the extra property that $t_{n} \in P$ when $n \equiv 1(\bmod 8)$ and $t_{n} \in Q$ when $n \equiv 5(\bmod 8)$; such a choice is possible. This ensures that $a \in P^{*}$ and $b \in Q^{*}$.

(ii) We choose $a$ and $b$ so that they are $P$-points in $A^{*}$ and $B^{*}$ respectively; this is possible by Theorem 5.5. Then $a$ and $b$ are $P$ points in $S^{*}$.

Now suppose that $S$ is an infinite, countable semigroup such that $S$ is just weakly cancellative, but may not be nearly right cancellative. Then the proof in Theorem 12.7 can be carried through, save that condition (ii) that arises in that proof need not hold. Thus we cannot be sure that the elements $a$ and $b$ which are constructed are right cancellable in $\beta S$. Taking account of the first remark after Theorem 12.5, we see that we do have the following closely related result.

THEOREM 12.9. Let $S$ be an infinite, countable, weakly cancellative semigroup. Then there exist $a$ and $b$ in $S^{*}$ that are right cancellable in $(\beta S, \square)$ and such that $\{a, b\}$ is determining for the left topological centre of $\beta S$. Further, $S$ is strongly Arens irregular.

Proof. The above remarks imply that $\{a, b\}$ is determining for the left topological centre of $\beta S$, and so $S$ is left strongly Arens irregular. 
The semigroup $S^{\text {op }}$ also satisfies the same conditions as $S$, and so $S^{\text {op }}$ is left strongly Arens irregular and $S$ is right strongly Arens irregular. Hence $S$ is strongly Arens irregular.

We see from Example 7.33 that the semigroup $S=\mathbb{N}_{\wedge}$ is not weakly cancellative, but nevertheless any two-element subset of $S^{*}$ is determining for the left topological centre of $\ell^{1}(S)$. An easy example of a semigroup $S$ with $\ell^{1}(S)$ Arens regular was given in Example 7.30.

We wish to enquire which special pairs $\{a, b\}$ are determining for the left topological centre of $\beta S$ or of $M(\beta S)$; for our results, we must restrict consideration to abelian semigroups. The following theorem will provide some examples

THEOREM 12.10. Let $S$ be an infinite, weakly cancellative, abelian semigroup, and let $\Omega$ be a non-empty, compact space. Let $\varphi: \beta S \rightarrow \Omega$ be a continuous function such that

$$
\varphi(u \square v)=\varphi(v) \quad\left(u \in \beta S, v \in S^{*}\right) .
$$

Take $a, b \in S^{*}$ with $\varphi(a) \neq \varphi(b)$. Then $\{a, b\}$ is determining for the topological centre of $\beta S$. Suppose, further, that $a$ and $b$ are right cancellable. Then $\{a, b\}$ is determining for the topological centre of $M(\beta S)$.

Proof. Take $\mu \in M(\beta S)$ such that $\mu \square a=\mu \diamond a$ and $\mu \square b=\mu \diamond b$.

Set $K=\left\{u \in S^{*}: \varphi(u)=\varphi(a)\right\}$ and $W=S^{*} \backslash K$, so that $K$ is compact and $W$ is a non-empty, open subset of $S^{*}$. Further, it follows from (12.8) that $K$ and $W$ are left ideals in $\beta S$, noting that $S^{*}$ is an ideal in $\beta S$. Take $\varepsilon>0$, and choose a compact subset $L \subset W$ such that $b \in L$ and $\|\mu|L-\mu| W\|<\varepsilon$. We may suppose that $L=\varphi^{-1}(\varphi(L))$, and hence that $L$ is a left ideal in $\beta S$. We see that $\operatorname{supp}(\nu \square a) \subset K$ and $\operatorname{supp}(\nu \square b) \subset L$ for each $\nu \in M(\beta S))$.

Set $\mu_{1}=\mu \mid K$ and $\mu_{2}=\mu \mid L$. Then $\operatorname{supp}\left(a \square \mu_{1}\right) \subset K$, $\operatorname{supp}(a \square \mu)=\operatorname{supp}(\mu \square a) \subset K$, and $\operatorname{supp}\left(a \square \mu_{2}\right) \subset L$. We have

$$
\left\|\mu \square a-\left(a \square \mu_{1}+a \square \mu_{2}\right)\right\|<\varepsilon,
$$

and so $\left\|a \square \mu_{2}\right\|<\varepsilon$. Similarly, $\left\|b \square \mu_{1}\right\|<\varepsilon$.

Suppose that either $\mu \in M(\beta S)^{+}$or that $a$ and $b$ are right cancellable. By Proposition 4.4, $\|\mu\|=\|\mu \square a\|=\|\mu \square b\|$, and so

$$
\|\mu\| \leq\left\|\mu_{1}\right\|+2 \varepsilon, \quad\|\mu\| \leq\left\|\mu_{2}\right\|+2 \varepsilon .
$$

Hence $2\|\mu\| \leq\left\|\mu_{1}\right\|+\left\|\mu_{2}\right\|+4 \varepsilon \leq\|\mu\|+4 \varepsilon$, and so $\mu=0$.

The result follows.

We shall apply the above with a special choice of $\varphi, S$, and $\Omega$. 
Indeed, we shall first prove the following remark, taken from [88]. Let $f: \mathbb{R} \rightarrow \mathbb{R}^{+}$be a uniformly continuous function such that

$$
\lim _{|t| \rightarrow \infty}|f(s+t)-f(t)|=0 \quad(s \in \mathbb{R}) .
$$

Set $X=U C(\mathbb{R})$. We regard $\mathbb{R}^{+}$as a subset of $\Phi_{X}$ and $f$ as a continuous map from $\mathbb{R}$ to $\Phi_{X}$, and then we extend $f$ to a continuous map, still called $f$, from $\Phi_{X}$ into itself.

Now fix $s \in \mathbb{R}$. For each $\lambda \in X$ and $\varepsilon>0$, choose $\delta>0$ such that $|\lambda(x)-\lambda(y)|<\varepsilon$ whenever $x, y \in \mathbb{R}$ and $|x-y|<\delta$, and then choose $m \in \mathbb{R}^{+}$such that $|f(s+t)-f(t)|<\delta$ whenever $|t|>m$. Then

$$
|(\lambda \circ f)(s+t)-(\lambda \circ f)(t)|<\varepsilon
$$

whenever $|t|>m$. We now regard $\lambda \circ f$ as an element of $C\left(\Phi_{X}\right)$. For each $u \in \Phi_{X}^{*}$, we have

$$
|(\lambda \circ f)(s \square u)-(\lambda \circ f)(u)|<\varepsilon .
$$

It follows that $f(s \square u)=f(u)$ as points of $\Phi_{X}$.

In particular, set $f(x)=\log (|x|+1) \quad(x \in \mathbb{R})$, so that $f$ satisfies equation (12.9). Set $\varphi=f \mid \mathbb{N}$, and extend $\varphi$ to a continuous function $\varphi: \beta \mathbb{N} \rightarrow \Phi_{X}$. Then $\varphi$ satisfies equation (12.8), taking $S=\mathbb{N}$ and $\Omega=\Phi_{X}$. Since $\varphi(\mathbb{N})$ contains an unbounded sequence in $\mathbb{R}^{+}$, the set $\varphi(\beta \mathbb{N}) \cap \Phi_{X}^{*}$ is infinite.

The above function $f$ is a 'slowly oscillating function' in the sense of $[\mathbf{4 5}]$; the arguments of $[\mathbf{4 5}]$ are related to those above.

In the corollary below, we shall use the following notation. Let $S=(\mathbb{N}, \cdot)$, and let $u \in \beta \mathbb{N}$. Then we write $2 \cdot u$ for the appropriate element in $(\beta S, \square)$. It is easily seen [78, Lemma 13.1] that

$$
n \cdot(u \square v)=(n \cdot u) \square(n \cdot v) \quad(n \in \mathbb{N}, u, v \in \beta \mathbb{N}) .
$$

Corollary 12.11. (i) For each $a \in \mathbb{N}^{*},\{a, 2 \cdot a\}$ is determining for the topological centre of $\beta \mathbb{N}$.

(ii) There exist minimal idempotents $p$ and $q$ in $\mathbb{N}^{*}$ such that $\{p, q\}$ is determining for the topological centre of $\beta \mathbb{N}$.

(iii) For each $a \in \mathbb{N}^{*}$ such that $a$ is right cancellable, $\{a, 2 \cdot a\}$ is determining for the topological centre of $M(\beta \mathbb{N})$.

(iv) There exist $a, b \in \overline{E(K(\beta \mathbb{N}))}$ such that $\{a, b\}$ is determining for the topological centre of $M(\beta \mathbb{N})$.

(v) There exist $a, b \in \mathbb{N}_{[\infty]}^{*}$ such that $\{a, b\}$ is determining for the topological centre of $M(\beta \mathbb{N})$. 
Proof. (i) Take $a \in \mathbb{N}^{*}$ such that $a$ is right cancellable, and set $b=2 \cdot a$. Take $\varphi$ to be as above. Clearly $\varphi(b)=\log 2+\varphi(a)$, and so $\varphi(b) \neq \varphi(a)$. By Theorem 12.10, $\{a, 2 \cdot a\}$ is determining for the topological centre of $M(\beta \mathbb{N})$.

(ii) Choose $p \in \mathbb{N}^{*}$ to be a minimal idempotent, so that, by Theorem 3.26 and Proposition 6.41, $p \in K\left(\mathbb{N}^{*}\right) \cap \mathbb{H}$. Set $q=2 \cdot p$. By (12.10), $q$ is an idempotent. To see that $q$ is also minimal, we first note that there exists $u \in \mathbb{N}^{*}$ with $u \square u=p$ because $p \in \mathbb{H}$. Now $\mathbb{N}^{*} \square u \square \mathbb{N}^{*}$ is an ideal in $\mathbb{N}^{*}$, and so $K\left(\mathbb{N}^{*}\right) \subset \mathbb{N}^{*} \square u \square \mathbb{N}^{*}$. Thus

$$
q=2 \cdot p \in \mathbb{N}^{*} \square p \square \mathbb{N}^{*} \subset K\left(\mathbb{N}^{*}\right) .
$$

Again by Theorem 3.26, $q$ is a minimal idempotent.

(iii) Let $a$ and $b$ be as in (i), with $a$ right cancellable. We show that $b$ is also right cancellable in $\mathbb{N}^{*}$. For suppose that $u, v \in \beta \mathbb{N}$ with $u \square b=v \square b$, and let $\pi$ be the continuous extension of the quotient map $\mathbb{N} \rightarrow \mathbb{Z}_{2}$. Then $\pi(b)=0$, and so $\pi(u)=\pi(v)$. If $\pi(u)=1$, replace $u$ and $v$ by $u \square 1$ and $v \square 1$, respectively. Thus we may suppose that $\pi(u)=\pi(v)=0$, and so there exist $u_{1}$ and $v_{1}$ in $\beta \mathbb{N}$ with $2 \cdot u_{1}=u$ and $2 \cdot v_{1}=v$. Hence $u_{1} \square a=u_{2} \square b$, and so $u_{1}=v_{1}$ and $u=v$, as required.

By Theorem 12.10, $\{a, 2 \cdot a\}$ is determining for the topological centre of $M(\beta \mathbb{N})$.

(iv) Now chose $a \in \overline{E(K(\beta \mathbb{N}))}$ such that $a$ is right cancellable; such an element $a$ exists by Proposition 6.20(ii). By Proposition 6.22, $2 \cdot a \in \overline{E(K(\beta \mathbb{N}))}$. By (iii), $\{a, 2 \cdot a\}$ is determining for the topological centre of $M(\beta \mathbb{N})$.

(v) By Proposition 6.38(ii), there exists $a$ in $\mathbb{N}_{[\infty]}^{*}$ such that $a$ is right cancellable. Then clearly $2 \cdot a \in \mathbb{N}_{[\infty]}^{*}$. By (iii), $\{a, 2 \cdot a\}$ is determining for the topological centre of $M(\beta \mathbb{N})$.

The most interesting question that remains open seems to be the following. Can we find $a$ and $b$ in $K(\beta \mathbb{N})$ or $E\left(\mathbb{N}^{*}\right)$ such that $\{a, b\}$ is determining for the topological centre of $M(\beta \mathbb{N})$ ? We shall now show that we can find a 4 -element subset of $E\left(\mathbb{N}^{*}\right)$ which is determining for the topological centre of $M(\beta \mathbb{N})$.

In the next two results, we continue to use the notation $X=U C(\mathbb{R})$ and $\varphi: \beta \mathbb{N} \rightarrow \Phi_{X}$, as above. By Proposition 6.15 (iii), there are elements in $\overline{K(\beta \mathbb{N})}$ which are right maximal in $E\left(\mathbb{Z}^{*}\right)$. 
Lemma 12.12. Let $q$ be right maximal in $E\left(\mathbb{Z}^{*}\right)$, and let $\mu \in M\left(\mathbb{N}^{*}\right)$ be such that $\|\mu\|=1$ and $\mu \square q=q \square \mu$. Set

$$
C=\left\{u \in \mathbb{Z}^{*}: u \square q=q\right\} \quad \text { and } \quad A=\{u \in \beta \mathbb{N}: \varphi(u) \in \varphi(C)\} .
$$

Then $\|\mu \mid A\| \geq 1 / 3$.

Proof. Note that $A$ is a left ideal in $\beta \mathbb{N}$ because $\varphi$ satisfies equation (12.8), and that $C \subset A$.

Set $B=\mathbb{N}^{*} \backslash A, \mu_{1}=\mu \mid A$, and $\mu_{2}=\mu \mid B$, so that $\mu=\mu_{1}+\mu_{2}$.

By Proposition 6.7(i), the set $C$ is finite, and $R_{q}$ is injective on $\beta \mathbb{N} \backslash C$, and hence on $B$. By Proposition 4.4(ii), $\left\|\mu_{2} \square q\right\|=\left\|\mu_{2}\right\|$.

Fix $\varepsilon>0$. Then there is a compact subset $K$ of $B$ such that $\left|\mu_{2}\right|(B \backslash K)<\varepsilon$. By replacing $K$ by $\varphi^{-1}(\varphi(K))$, we may suppose that $K$ is a left ideal in $\beta \mathbb{N}$. Set $\mu_{2}^{\prime}=\mu_{2} \mid K$. We have

$$
\operatorname{supp}(\mu \square q) \subset A, \quad \operatorname{supp}\left(q \square \mu_{1}\right) \subset A, \quad \text { and } \quad \operatorname{supp}\left(q \square \mu_{2}^{\prime}\right) \subset K .
$$

Since $\mu \square q=q \square \mu_{1}+q \square \mu_{2}$, we have $\left\|\mu \square q-q \square \mu_{1}-q \square \mu_{2}^{\prime}\right\|<\varepsilon$. Since

$$
\operatorname{supp}\left(\mu \square q-q \square \mu_{1}\right) \cap \operatorname{supp}\left(q \square \mu_{2}^{\prime}\right)=\emptyset,
$$

it follows that $\left\|q \square \mu_{2}^{\prime}\right\|<\varepsilon$, and hence $\left\|q \square \mu_{2}\right\|<2 \varepsilon$. This holds for each $\varepsilon>0$, and so $q \square \mu_{2}=0$.

Assume towards a contradiction that $\left\|\mu_{1}\right\|<1 / 3$. Clearly we have $\mu \square q=q \square \mu_{1}$, and so $\|\mu \square q\|<1 / 3$, whence

$$
\left\|\mu_{2}\right\|=\left\|\mu_{2} \square q\right\|=\left\|\mu \square q-\mu_{1} \square q\right\| \leq\|\mu \square q\|+\left\|\mu_{1} \square q\right\|<2 / 3
$$

and $\|\mu\|<1$, the required contradiction. This shows that $\left\|\mu_{1}\right\| \geq 1 / 3$, as required.

TheOrem 12.13. There exists a subset $V$ of $E\left(\mathbb{N}^{*}\right)$ with $|V|=4$ such that $V$ is determining for the topological centre of $M(\beta \mathbb{N})$.

Proof. Let $\varphi: \beta \mathbb{N} \rightarrow \Phi_{X}$ be as above. Since $\varphi(\beta \mathbb{N}) \cap \Phi_{X}^{*}$ is infinite, there are four distinct points, say $x_{1}, x_{2}, x_{3}, x_{4}$, in $\varphi(\beta \mathbb{N}) \cap \Phi_{X}^{*}$. Let $U_{1}, U_{2}, U_{3}, U_{4}$ be pairwise disjoint, open subsets of $\Phi_{X}$ such that $x_{j} \in U_{j}\left(j \in \mathbb{N}_{4}\right)$. Since $\varphi$ is continuous and satisfies (12.8), each $\varphi^{-1}\left(U_{j}\right)$ is a non-empty, open subset of $\mathbb{N}^{*}$ and a left ideal, and so, by Theorem 3.25, each $\varphi^{-1}\left(U_{j}\right)$ contains an idempotent; by Proposition 6.7(ii), there is a right maximal idempotent, say $p_{1}$, in $\varphi^{-1}\left(U_{1}\right)$.

For each $m \in \mathbb{N}$, the element $m \cdot p$ is also a right maximal idempotent, and so we obtain right maximal idempotents $p_{2}=m_{2} \cdot p$, $p_{3}=m_{3} \cdot p$, and $p_{4}=m_{4} \cdot p$ in $\varphi^{-1}\left(U_{2}\right), \varphi^{-1}\left(U_{3}\right), \varphi^{-1}\left(U_{4}\right)$, respectively, for suitable $m_{2}, m_{3}, m_{4} \in \mathbb{N}$.

For $j \in \mathbb{N}_{4}$, set

$$
C_{j}=\left\{u \in \beta \mathbb{N}: u \square p_{j}=p_{j}\right\} \quad \text { and } \quad A_{j}=\varphi^{-1}\left(\varphi\left(C_{j}\right)\right) .
$$


We have $\varphi\left(C_{j}\right)=\log m_{j}+\varphi\left(C_{1}\right) \quad(j=2,3,4)$, and so, by choosing $m_{2}, m_{3}, m_{4}$ appropriately, we may suppose that $A_{i} \cap A_{j}=\emptyset(i \neq j)$.

Assume towards a contradiction that there exists $\mu \in M\left(\mathbb{N}^{*}\right)$ with $\|\mu\|=1$ and such that $\mu \square p_{j}=p_{j} \square \mu\left(j \in \mathbb{N}_{4}\right)$. By the lemma, $\left\|\mu \mid A_{j}\right\| \geq 1 / 3\left(j \in \mathbb{N}_{4}\right)$, and so $\|\mu\| \geq 4 / 3$, a contradiction. Thus $V$ is determining for the topological centre of $M(\beta \mathbb{N})$.

Uncountable semigroups We now extend Theorem 12.7 to cover some uncountable semigroups; the proof is similar to that previously given, but the details are somewhat different.

THEOREM 12.14. Let $S$ be an infinite semigroup such that $S$ is weakly cancellative and nearly right cancellative. Then there exist a and $b$ in $U_{S}$ such that $a$ and $b$ are right cancellable in $\beta S$ and clauses (i)-(iii) of Theorem 12.5 are satisfied with $K=U_{S}$.

Proof. We may suppose that $S$ has an identity $e_{S}$. Set $\kappa=|S|$, and enumerate $S$ as $\left(s_{\alpha}: \alpha<\kappa\right)$, where $s_{0}=e_{S}$. For $\sigma<\kappa$, set $S_{\sigma}=\left\{s_{\alpha}: \alpha \leq \sigma\right\}$.

The case where $\kappa=\omega$ has been covered in the proof of Theorem 12.7, and so we may suppose that $\kappa>\omega$.

Since $S$ is nearly right cancellative, there is a subset $X \in[S]^{\kappa}$ such that, for each $s, t \in S$ with $s \neq t$, the set

$$
E_{s, t}:=\{x \in X: s x=t x\}
$$

is finite; we may suppose that $e_{S} \in X$. We shall construct a sequence $\left(t_{\alpha}: \alpha<\kappa\right)$ in $X$ with $t_{0}=s_{0}$.

Suppose that $\tau<\kappa$, and assume that $t_{\sigma}$ has been defined for each $\sigma<\tau$. Set

$$
T_{\tau}=\left\langle S_{\tau} \cup\left\{t_{\sigma}: \sigma<\tau\right\}\right\rangle,
$$

a subsemigroup of $S$. Since $\kappa>\omega$, we see that $\left|T_{\tau}\right| \leq \max \{\omega,|\tau|\}<\kappa$.

We define the subset $U_{\tau}$ of $S$ to consist of the elements $u \in S$ such that there are $x_{1}, x_{2}, y_{1}, y_{2} \in T_{\tau}$ and $\sigma<\tau$ such that $x_{1} u y_{1}=x_{2} t_{\sigma} y_{2}$. Thus $\left\{t_{\sigma}: \sigma<\tau\right\} \subset U_{\tau}$. Next the subset $V_{\tau}$ of $S$ consists of the elements $v \in S$ such that there are $x_{1}, x_{2}, y_{1}, y_{2}, u \in U_{\tau}$ such that $x_{1} v y_{1}=x_{2} u y_{2}$. We note that $\left|V_{\tau}\right| \leq \max \{\omega,|\sigma|\}<\kappa$ because $S$ is weakly cancellative. Further,

$$
\left|\bigcup\left\{E_{s, t}: s, t \in S_{\tau}, s \neq t\right\}\right|=|\tau|<\kappa,
$$

and so there exists $t_{\tau} \in X \backslash V_{\tau}$ such that $t_{\tau} \notin E_{s, t}$ whenever $s, t \in S_{\tau}$ with $s \neq t$. This continues the inductive construction of the sequence $\left(t_{\alpha}: \alpha<\kappa\right)$. 
Set

$$
T=\left\{t_{\alpha}: \alpha<\kappa\right\}
$$

For each $\tau<\kappa$, take $W_{\tau}$ to consist of the elements $w \in S$ such that $x_{1} w y_{1}=x_{2} t_{\tau} y_{2}$ for some $x_{1}, x_{2}, y_{1}, y_{2} \in T_{\tau}$. Then we claim that $U_{\tau} \cap W_{\tau}=\emptyset$. For assume that $u \in U_{\tau} \cap W_{\tau}$. Then $t_{\tau} \in V_{\tau}$, a contradiction of the choice of $t_{\tau}$. Thus the claim holds. In particular, $W_{\sigma} \cap W_{\tau}=\emptyset$ whenever $\sigma, \tau<\kappa$ with $\sigma \neq \tau$. Let $s=s_{\alpha} \in S$ and $w \in S$. Then, for each $\tau>\alpha$, we have $w \in W_{\tau}$ if and only if $w s \in W_{\tau}$.

Let $A$ be a subset of $T$ with $|A|=\kappa$, and let $a$ correspond to a uniform ultrafilter on $A$, so that $a \in A^{*} \cap U_{S}$; set $U=\bigcup\left\{W_{\alpha}: t_{\alpha} \in A\right\}$, so that $A \subset U$. For each $s \in S$, we have $s t_{\alpha} \in W_{\alpha}$ eventually; if also $t_{\alpha} \in A$, then $s t_{\alpha} \in U$. Thus $s \square a \in \bar{U}$, and so $S \square a \subset \bar{U}$.

We claim that $a$ is right cancellable in $\beta S$. Indeed, let $u_{1}$ and $u_{2}$ be distinct points of $\beta S$, and take disjoint subsets $N_{1}$ and $N_{2}$ of $S$ such that $u_{j} \in \overline{N_{j}}(j=1,2)$. For $j=1,2$, set

$$
Y_{j}=\left\{s_{\alpha} t_{\sigma}: s_{\alpha} \in N_{j}, t_{\sigma} \in A, \alpha<\sigma<\kappa\right\},
$$

so that $Y_{j} \in u_{j} \square a$. As before, $Y_{1} \cap Y_{2}=\emptyset$. Thus $u_{1} \square a \neq u_{2} \square a$, as required.

Similarly, there exists a subset $B \in[T]^{\kappa}$, a set

$$
V=\bigcup\left\{W_{\alpha}: t_{\alpha} \in B\right\}
$$

and a corresponding point $b \in B^{*} \cap U_{S}$ such that $b$ is right cancellable in $\beta S$ and $S \square b \subseteq \bar{V}$. By taking $A$ and $B$ to be disjoint, we may suppose that $\bar{U} \cap \bar{V}=\emptyset$.

We claim that, for each $s \in S$ and each $x \in U_{S}$, we have $x \diamond s \in \bar{U}$ if and only if $x \in \bar{U}$. For suppose that $x \diamond s_{\alpha} \in \bar{U}$, where $\alpha<\kappa$. Then $\left\{u \in S: u s_{\alpha} \in U\right\} \in x$. Since $x$ is a uniform ultrafilter, it follows that

$$
\left\{u \in S: u s_{\alpha} \in \bigcup_{\beta>\alpha}\left\{W_{\beta}: t_{\beta} \in A\right\}\right\} \in x .
$$

But this set is contained in $\bigcup_{\beta>\alpha}\left\{W_{\beta}: t_{\beta} \in A\right\}$, and therefore it is contained in $U$. Thus $x \in \bar{U}$. Similarly, $x \diamond s \in \bar{V}$ if and only if $x \in \bar{V}$. Hence clause (iii) of Theorem 12.5 is satisfied.

Thus $a$ and $b$ have the required properties.

It follows from the theorem that the only element $\mu \in M\left(U_{S}\right)$ such that $\mu \square a=\mu \diamond a$ and $\mu \square b=\mu \diamond b$ is $\mu=0$; we shall obtain a stronger result in our main theorem, below.

THEOREM 12.15. Let $S$ be an infinite semigroup 
such that $S$ is weakly cancellative and nearly right cancellative. Then there exist $a$ and $b$ in $S^{*}$ that are right cancellable in $(\beta S, \square)$ and such that the two-element set $\{a, b\}$ is determining for the left topological centre of $M(\beta S)$.

Suppose that $S$ is cancellative and $P$ and $Q$ are subsets of $S$ with $|P|=|Q|=|S|$. Then we can take $a \in P^{*}$ and $b \in Q^{*}$.

Proof. Set $\kappa=|S|$. The case where $\kappa=\omega$ is covered in Theorem 12.7, and so we may suppose that $\kappa>\omega$.

We may suppose that $S$ has an identity $e_{S}$. Enumerate $S$ as a sequence $\left(s_{\alpha}: \alpha<\kappa\right)$, where $s_{0}=e_{S}$.

We follow the construction specified in Theorem 12.14 to obtain sets $U_{\tau}, V_{\tau}$, and $W_{\tau}$ for each $\tau<\kappa$, a set $T=\left\{t_{\alpha}: \alpha<\kappa\right\}$ in $S$, and two points $a, b$ in $U_{S}$. In particular, $t_{\tau} \notin V_{\tau}(\tau<\kappa)$. As before, $a$ and $b$ are right cancellable in $\beta S$. We shall use the fact, which is contained in the proof of Theorem 12.14, that an expression of an element in $S$ in the form $s_{\alpha} t_{\beta}$, where $\alpha<\beta<\kappa$, is unique.

In this case, $a$ corresponded to a uniform ultrafilter on a subset $A \in[T]^{\kappa}$. By Proposition 5.4, we may suppose that $A$ has the following additional property: for each infinite cardinal $\zeta<\kappa$, the set $A$ can be partitioned into $\zeta$ pairwise disjoint sets such that, for each $\eta<\zeta$, the union of each collection of $\eta$ sets of the partition is not a member of $a$. (This implies that $a$ cannot be a ' $\kappa$-complete' ultrafilter.)

We shall prove that $\{a, b\}$ is determining for the left topological centre of $M(\beta S)$. Let $\mu \in M\left(S^{*}\right)$ have the property that

$$
\mu \square a=\mu \diamond a \quad \text { and } \quad \mu \square b=\mu \diamond b .
$$

We assume towards a contradiction that $\mu \neq 0$.

We first write $\mu=\mu_{1}+\mu_{2}$, where $\mu_{1}=\mu \mid\left(\beta S \backslash U_{S}\right)$ and $\mu_{2}=\mu \mid U_{S}$, so that $\|\mu\|=\left\|\mu_{1}\right\|+\left\|\mu_{2}\right\|$. Take $\varepsilon>0$. By Theorems 12.14 and 12.5 (with $K=U_{S}$ ), there exist $x \in\{a, b\}$ and $\lambda \in C(\beta S)_{[1]}$ such that

$$
\langle\mu \square x, \lambda\rangle>\left\|\mu_{1}\right\|+\left\|\mu_{2}\right\|-\varepsilon \quad \text { and } \quad\left|\left\langle\mu_{2} \diamond x, \lambda\right\rangle\right| \leq \frac{1}{2}\left\|\mu_{2}\right\| .
$$

It follows that

$$
|\langle\mu \diamond x, \lambda\rangle| \leq\left\|\mu_{1}\right\|+\frac{1}{2}\left\|\mu_{2}\right\| .
$$

Since $\mu \square x=\mu \diamond x$, we have $\left\|\mu_{2}\right\| / 2 \leq \varepsilon$. This holds for each $\varepsilon>0$, and so $\mu_{2}=0$. Thus $\mu \mid U_{S}=0$. Since $|\mu|$ is a regular measure, there is a compact subset $C$ of $\beta S \backslash U_{S}$ such that $|\mu|(C)>0$, and so there is a set $Y \in[S]^{<\kappa}$ such that $|\mu|(\bar{Y})>0$.

Let $Y$ be a subset of $S$ of minimum cardinality with $|\mu|(\bar{Y})>0$; say $|Y|=\zeta$. Necessarily $\zeta$ is infinite, and we have seen that $\zeta<\kappa$. 
Enumerate $Y$ as $\left(y_{\iota}: \iota<\zeta\right)$. For $\sigma<\kappa$, set

$$
\begin{aligned}
& I_{\sigma}=\left\{y \in Y: y t_{\sigma}=s_{\alpha} t_{\beta} \text { for some } \alpha<\beta<\sigma\right\}, \\
& J_{\sigma}=\left\{y \in Y: y t_{\beta}=s_{\alpha} t_{\sigma} \text { for some } \alpha<\beta<\sigma\right\} .
\end{aligned}
$$

Take $y \in I_{\sigma}$. Then $y \in U_{\sigma+1}$, and so $y \notin I_{\tau}$ for any $\tau>\sigma$ because this would imply that $t_{\tau} \in U_{\sigma+1}^{-1} T_{\sigma}^{2} \subset V_{\tau}$, and this is not the case by the choice of $t_{\tau}$. Thus $I_{\sigma} \cap I_{\sigma+1}=\emptyset$. Set

$$
I=\left\{t_{\sigma}: \sigma<\kappa, I_{\sigma} \neq \emptyset\right\}, \quad J=\left\{t_{\sigma}: \sigma<\kappa, J_{\sigma} \neq \emptyset\right\} .
$$

Then it follows that $|I| \leq|Y|=\zeta<\kappa$. Similarly, $|J|<\kappa$. Now set $W=A \backslash(I \cup J)$. Then $W \in a$ because $a \in U_{S}$. Suppose that $y t=s_{\alpha} t_{\beta}$ where $y \in Y, t, t_{\beta} \in W$, and $\alpha<\beta<\kappa$. Then necessarily $\sigma=\beta$.

By our choice of the point $a \in U_{S}$, the set $W$ can be partitioned into pairwise disjoint sets $E_{\iota}$ for $\iota<\zeta$ such that, for each $\eta<\zeta$, the union of $\eta$ of the sets $E_{\iota}$ is not a member of $a$. Define a function $\varphi: W \rightarrow \zeta$ by setting $\varphi(t)=\iota$ whenever $t \in E_{\iota}$.

Now define

$$
Z=\left\{y_{\iota} t: t \in W \text { with } \varphi(t)<\iota\right\} .
$$

Let $v \in U_{Y}$. For each $t \in W$, the set $\left\{y_{\iota}: \iota>\varphi(t)\right\}$ belongs to $v$, and so $Z \in v \diamond a$. Thus $U_{Y} \diamond a \subset \bar{Z}$.

However, we claim that $Z \notin u \square a$ for each $u \in \beta S$. For take $u \in \beta S$ and assume that $Z \in u \square a$. By Proposition 6.4, (a) $\Rightarrow(\mathrm{c}), Z$ contains a set of the form $s_{\alpha} R$ for some $\alpha<\kappa$ and some $R \in a$ such that $R \subset\left\{t_{\beta}: \beta>\alpha\right\} \cap W$. Fix such an $\alpha$, and let $s_{\alpha} t_{\beta} \in Z$, where $\alpha<\beta<\kappa$ and $t_{\beta} \in W$. Then $s_{\alpha} t_{\beta}=y_{\iota} t$ for some $t \in W$ with $\varphi(t)<\iota<\zeta$. This implies that $t=t_{\beta}$, and so $s_{\alpha} t_{\beta}=y_{\iota} t_{\beta}$. If $y_{\iota} \neq s_{\alpha}$, then $\left\{t \in T: s_{\alpha} t=y_{\iota} t\right\}$ is finite because $T$ was chosen to be a subset of the witness set $X$, and so the set

$$
R_{0}:=\left\{t \in T: s_{\alpha} t=y t \text { for some } y \in Y \text { with } y \neq s_{\alpha}\right\}
$$

has cardinality at most $|Y|=\zeta<\kappa$. Thus $R \backslash R_{0} \in a$. For each $t \in R \backslash R_{0}$, we have $s_{\alpha} t=y_{\iota} t$ for a unique $y_{\iota}=s_{\alpha} \in Y$, and, in this case, $\varphi(t)<\iota$. But, for each $\iota<\zeta$, we have

$$
\{t \in W: \varphi(t)<\iota\} \subset \bigcup\left\{E_{\eta}: \eta<\iota\right\},
$$

and the set on the right-hand side does not belong to $a$. This contradiction shows that $Z \notin u \square a$, as claimed. Hence $(\beta S \square a) \cap \bar{Z}=\emptyset$.

Let $\nu$ denote the restriction of $\mu$ to the compact set $U_{Y}$. For each $\varepsilon>0$, there exists $\lambda \in C(\beta S)_{[1]}$ such that

$$
\langle\mu \square a, \lambda\rangle>\|\mu\|-\varepsilon=\|\nu\|+\|\mu-\nu\|-\varepsilon .
$$


We may suppose that $(\operatorname{supp} \lambda) \cap \bar{Z}=\emptyset$, and hence that $\langle\nu \diamond a, \lambda\rangle=0$, which implies that $|\langle\mu \diamond a, \lambda\rangle| \leq\|\mu-\nu\|$. Since $\mu \square a=\mu \diamond a$, it follows that $\|\nu\|<\varepsilon$. But this holds for each $\varepsilon>0$, and so $\nu=0$.

Since $|\mu|(\bar{Y})>0$ and $\mu \mid U_{Y}=0$, it follows that there exists $Y_{0} \subset Y$ such that $\left|Y_{0}\right|<|Y|$ and $|\mu|\left(\overline{Y_{0}}\right)>0$. But this contradicts the fact that $Y$ is a subset of $S$ of minimum cardinality with $|\mu|(\bar{Y})>0$. Hence $\mu=0$, as required.

Corollary 12.16. Let $S$ be a weakly cancellative and nearly cancellative semigroup. Then $\ell^{1}(S)$ is strongly Arens irregular.

Corollary 12.17. Let $S=\mathcal{M}^{\circ}(G, P, n)$, where $G$ is a group and $P$ is an invertible sandwich matrix in $\mathbb{M}_{n}\left(\ell^{1}(G)\right)$. Then there is a subset $V$ of $S^{*}$ of cardinality $2 n$ such that $V$ is determining for the left topological centre of $M(\beta S)$; further, in the case where $G$ is abelian, $2 n$ is the minimum cardinality of such a set $V$.

Proof. By Theorem 4.16, we may suppose that $P$ is the identity matrix, so that $\ell^{1}(S)=\mathcal{M}^{o}\left(\ell^{1}(G), n\right)$. By Theorem 12.15, there exist $a, b \in G^{*}$ such that $\{a, b\}$ is determining for the left topological centre of $M(\beta G)$. Set $a_{i}=(a)_{i i}$ and $b_{i}=(b)_{i i}$ for $i \in \mathbb{N}_{n}$, and then set $V=\left\{a_{1}, \ldots, a_{n}, b_{1}, \ldots, b_{n}\right\}$, so that $V \subset S^{*}$ and $|V|=2 n$. It is easily checked (by matrix multiplication) that $V$ is determining for the left topological centre of $M(\beta S)$.

In the case where $G$ is abelian, the minimum size of a subset of $G^{*}$ that is determining for the left topological centre of $M(\beta G)$ is 2 . By considering 'diagonal' elements of $\mathbb{M}_{n}(M(G))$, we see that $2 n$ is the minimum cardinality of such a set $V$.

Corollary 12.18. Let $S$ be a semigroup such that the Banach algebra $\ell^{1}(S)$ is amenable. Then there is a finite subset of $S^{*}$ which is determining for the left topological centre of $M(\beta S)$. Further, $\ell^{1}(S)$ is strongly Arens irregular.

Proof. By Theorem 10.12, $S$ has the structure described in that theorem. Let $T$ be a semigroup which is a quotient of the form $I_{j} / I_{j+1}$ or of the form $K(S)$. By the above corollary, there is a finite subset of $T^{*}$ which is determining for the left topological centre of $M(\beta T)$. By a small modification of the proof of Theorem 2.25, we obtain a finite subset of $S^{*}$ which is determining for the left topological centre of $M(\beta S)$. It follows that $\ell^{1}(S)$ is left strongly Arens irregular, and hence strongly Arens irregular by symmetry. 
EXAmple 12.19. Let $S=\left(\bigcup_{j \in \mathbb{N}} S_{j}\right) \cup\{o\}$ be an abelian semigroup, where $\left\{S_{j}: j \in \mathbb{N}\right\}$ is a countable family of pairwise disjoint infinite subgroups and $S_{i} S_{j}=\{o\}$ when $i \neq j$; such a semigroup is specified in Example 3.34. Let $V$ be a subset of $S^{*}$ such that $V$ is determining for the topological centre of $\beta S$, and let $i \in \mathbb{N}$. We claim that $V \cap \overline{S_{i}} \neq \emptyset$. Indeed, set $T=S \backslash S_{i}$, and assume that $V \subset \bar{T}$. Then $S_{i} T=\{o\}$, and so $u \square v=u \diamond v=o$ for each $u \in \overline{S_{i}}$ and $v \in V$, a contradiction. It follows that $V$ is an infinite set.

The above theorem can be modified slightly as before to establish the following result.

THEOREM 12.20. Let $S$ be an infinite, weakly cancellative semigroup.

Then there is subset $V$ of $S^{*}$ of cardinality 2 such that $V$ is determining for the left topological centre of $\beta S, S$ is strongly Arens irregular, and the map $L_{v}: u \mapsto v \square u, \beta S \rightarrow \beta S$, is continuous if and only if $v \in S$.

ExAMPLE 12.21. In Example 7.34, we presented an infinite, countable, weakly cancellative semigroup for which $\ell^{1}(S)$ is not strongly Arens irregular. By the theorem, $S$ is strongly Arens irregular.

EXAMPLE 12.22. At the beginning of this chapter, we raised the possibility that, for certain semigroups $S$, a singleton could be determining for the left topological centre of $M(\beta S)$. We now give an example to show that this situation can arise.

Let $S=\mathbb{S}_{2}$ be the free semigroup on two symbols, $a$ and $b$, and let $|w|$ be the length of a word $w$ in $S$. Set $U=\left\{b a^{n}: n \in \mathbb{N}\right\}$, and choose $x \in U^{*}$ such that $x$ is right cancellable in both $(\beta S, \square)$ and $(\beta S, \diamond)$; by Proposition 6.20(iv) such a choice is possible.

Set

$$
\begin{aligned}
& A=\left\{t b a^{n} \in S: t \in S, n \in \mathbb{N}, \text { and }|t|<n\right\}, \\
& B=\left\{t b a^{n} \in S: t \in S, n \in \mathbb{N}, \text { and }|t|>n\right\}
\end{aligned}
$$

so that $A$ and $B$ are disjoint subsets of $S$, and hence $\bar{A} \cap \bar{B}=\emptyset$. Clearly $S^{*} \square x \subset \bar{A}$ and $S^{*} \diamond x \subset \bar{B}$.

Now assume that there exists $\mu \in M\left(S^{*}\right)$ such that $\|\mu\|=1$ and

$$
\|\mu \square x-\mu \diamond x\|<1 .
$$

Since $\operatorname{supp}(\mu \square x) \subset \bar{A}$ and $\operatorname{supp}(\mu \diamond x) \subset \bar{B}$, we have

$$
\|\mu \square x-\mu \diamond x\|=\|\mu \square x\|+\|\mu \diamond x\| \text {. }
$$


Since $x$ is right cancellable in $(\beta S, \square)$, it follows from Proposition 4.4(iii) that $\|\mu \square x\|=\|\mu\|=1$. Thus we have a contradiction, and so the only $\mu$ in $M\left(S^{*}\right)$ satisfying (12.11) is $\mu=0$. In particular, $\{x\}$ is determining for the left topological centre.

We can modify the above slightly to see that the free group $\mathbb{F}_{2}$ has the same property. Indeed let $x \in \mathbb{F}_{2}$ have the form

$$
x=a^{m_{1}} b^{n_{1}} \cdots a^{m_{k}} b^{n_{k}},
$$

as in equation (3.7); we define $d(x)=n_{k}$, and set

$$
A=\left\{x \in \mathbb{F}_{2}:|x|<2 d(x)\right\}, \quad B=\left\{x \in \mathbb{F}_{2}:|x|>2 d(x)\right\},
$$

so that $A \cap B=\emptyset$. Set $C=\left\{b^{n} a^{n}: n \in \mathbb{N}\right\}$, and choose $v \in C^{*}$.

We claim that, for each $u \in \mathbb{F}_{2}^{*}$, we have $u \square v \in \bar{A}$ and $u \diamond v \in \bar{B}$. It is obvious that $u \square v \in \bar{A}$. Now take $\left(x_{\alpha}\right)$ in $\mathbb{F}_{2}$ and $\left(y_{\beta}\right)$ in $C$ such that $u=\lim _{\alpha} x_{\alpha}$ and $v=\lim _{\beta} y_{\beta}$, and then $u \diamond v=\lim _{\beta} \lim _{\alpha} x_{\alpha} y_{\beta}$. We may suppose by passing to a subnet that either $\lim _{\alpha} d\left(x_{\alpha}\right)=-\infty$ or that there exists $m \in \mathbb{N}$ such that $d\left(x_{\alpha}\right) \geq-m$ for each $\alpha$. The claim is again clear in the former case. In the latter case we can suppose that $y_{\beta}$ has the form $b^{n_{\beta}} a^{n_{\beta}}$ with $n_{\beta}>m$ for each $\beta$, and then, once again, the claim holds.

We claim that $v$ is right cancellable in $\left(\beta \mathbb{F}_{2}, \square\right)$. Indeed, assume towards a contradiction that there exist $u_{1}, u_{2}$ in $\mathbb{F}_{2}^{*}$ with $u_{1} \neq u_{2}$ such that $u_{1} \square v=u_{2} \square v$. For $j=1,2$, choose $U_{j} \in u_{j}$ such that $U_{1} \cap U_{2}=\emptyset$; we have

$$
\left\{x b^{n} a^{n}: x \in U_{j}, n=d\left(x b^{n} a^{n}\right)\right\} \in u_{j} \square v,
$$

and so there exist $x_{j} \in U_{j}$ and $n_{j} \in \mathbb{N}$ such that $x_{1} b^{n_{1}} a_{1}^{n}=x_{2} b^{n_{2}} a_{2}^{n}$ and $n_{j}=d\left(x_{j} b^{n_{j}} a^{n_{j}}\right)$. But now $n_{1}=n_{2}$, and hence $x_{1}=x_{2}$, a contradiction because $U_{1} \cap U_{2}=\emptyset$. Hence the claim holds.

Again $\{v\}$ is determining for the left topological centre of $M\left(\mathbb{F}_{2}\right)$.

In fact, for each $\mu \in M\left(\mathbb{F}_{2}\right) \backslash \ell^{1}\left(\mathbb{F}_{2}\right)$, we have $\operatorname{supp}(\mu \square v) \subset \bar{A}$, $\operatorname{supp}(\mu \diamond v) \subset \bar{B}$, and $\|\mu \square v\|=\|\mu\|$, and so $\|\mu \square v-\mu \diamond v\| \geq\|\mu\|$. This implies that $v$ is such that $\mu \in \ell^{1}\left(\mathbb{F}_{2}\right)$ whenever $\mu \in M\left(\mathbb{F}_{2}\right)$ and $\|\mu \square v-\mu \diamond v\|<\|\mu\|$.

By replacing the above element $v$ by an idempotent in $\beta \mathbb{F}_{2} \square v$, we find an idempotent $p$ which is determining for the left topological centre of $\beta \mathbb{F}_{2}$. However we cannot deduce that $p$ is determining for the left topological centre of $M\left(\beta \mathbb{F}_{2}\right)$ because $p$ is not right cancellable in $\beta \mathbb{F}_{2}$.

We do not know an example of an amenable semigroup $S$ such that a singleton is determining for the left topological centre of $M(\beta S)$. 
Let $S$ be a semigroup, and let $v \in \beta S$. Then one can ask whether, for $u \in \beta S$, the continuity of $L_{u}: \beta S \rightarrow \beta S$ at $v$ forces $u$ to belong to $S$. (This is a stronger hypothesis than that $\{v\}$ is determining for the left topological centre of $\beta S$.) Such a point $v$ has been exhibited by Protasov [117, Theorem 1] in the case where $S$ is a countable group.

Locally compact groups The final theorem of this chapter applies to locally compact, rather than discrete, groups. It is analogous to Theorem 12.7, and the proof uses a similar idea.

Let $G$ be a locally compact group. For an introduction to the theory of the $C^{*}$-algebra $X=L U C(G)$, see Chapter 8. In particular, we have $M\left(\Phi_{X}\right)=M(G) \ltimes E^{\circ}$, where $E=C_{0}(G)$, and we identify $E^{\circ}$ with $M\left(\Phi_{X}^{*}\right)$.

We remark that the proof of the following theorem is considerably easier in the case where $G$ is also assumed to be $\sigma$-compact.

Definition 12.23. Let $G$ be a locally compact group, let $X$ be a left-introverted $C^{*}$-subalgebra of $L^{\infty}(G)$, let $v \in \Phi_{X}$, and let $Y$ be a topological space. A function $f: \Phi_{X} \rightarrow Y$ is $G$-continuous at $v$ if $\lim _{t \rightarrow v} f(t)=f(v)$, where we require that $t \in G$ in the limiting process.

Thus, in the case where $X$ is introverted, $L_{\mu}: \Phi_{X} \rightarrow M\left(\Phi_{X}\right)$ is $G$-continuous at $v \in \Phi_{X}$ if and only if $\mu \square v=\mu \diamond v$.

THEOREM 12.24. Let $G$ be a locally compact, non-compact group, and set $X=L U C(G)$. Then there is a 2-element subset $V$ of $\Phi_{X}$ such that

$M(G)=\left\{\mu \in M\left(\Phi_{X}\right): L_{\mu} \mid \Phi_{X}\right.$ is $G$-continuous at each point of $\left.V\right\}$.

Proof. Let $U$ be a fixed compact, symmetric neighbourhood of $e_{G}$ in $G$, and let $\left(K_{\alpha}: \alpha<\kappa\right)$ be a family of compact subspaces of $G$ such that $U \subset K_{\alpha}(\alpha<\kappa)$, such that $\bigcup\left\{K_{\alpha}: \alpha<\kappa\right\}=G$, and such that $\kappa$ is the minimum cardinality of such a family (so that $\kappa$ is the compact covering number of $G$ ).

We shall inductively choose a $\kappa$-sequence $\left(t_{\alpha}: \alpha<\kappa\right)$ of points of $G$ and an increasing $\kappa$-sequence $\left(E_{\alpha}: \alpha<\kappa\right)$ of subsets of $G$ such that the following properties hold for each $\alpha<\kappa$ :

(i) $E_{\alpha}$ can be covered by $|\alpha|$ compact subsets of $G$;

(ii) $U \subset K_{\alpha} \subset E_{\alpha}$;

(iii) $\bigcup\left\{U E_{\beta}: \beta<\alpha\right\} \subset E_{\alpha}$;

(iv) $\bigcup\left\{E_{\beta} t_{\gamma} \cup E_{\beta} t_{\gamma}^{-1}: \gamma \leq \beta<\alpha\right\} \subset E_{\alpha}$;

(v) $t_{\alpha} \notin \bigcup\left\{E_{\beta} t_{\gamma}: \gamma \leq \beta<\alpha\right\}$; 
(vi) $t_{\alpha} \notin \bigcup\left\{E_{\beta}^{-1} E_{\beta}: \beta<\alpha\right\}$.

Indeed, set $E_{0}=K_{0}$ and $t_{0}=e_{G}$. Now suppose that $0<\alpha<\kappa$, and assume that $t_{\beta}$ and $E_{\beta}$ have been specified for each $\beta<\alpha$. We choose $E_{\alpha}$ to satisfy (i)-(iv), and then choose $t_{\alpha}$ to satisfy (v) and (vi); clearly such choices are possible. This continues the inductive construction.

Certainly we have $\bigcup\left\{E_{\alpha}: \alpha<\kappa\right\}=G$. Set $T=\left\{t_{\alpha}: \alpha<\kappa\right\}$.

Take $\alpha<\kappa$. Then $U E_{\alpha} \subset E_{\alpha+1}$ by the construction, and so we have $\overline{U E_{\alpha}} \subset \overline{E_{\alpha+1}}$ in $\Phi_{X}$. By the continuity of the multiplication, $U \overline{E_{\alpha}}=\overline{U E_{\alpha}}$. By Proposition 8.11(iii), $U \overline{E_{\alpha}}$ is a neighbourhood of $\overline{E_{\alpha}}$. Hence we see that

$$
\overline{E_{\alpha}} \subset \operatorname{int} \overline{E_{\alpha+1}}
$$

We define

$$
E=\bigcap_{\alpha<\kappa} \overline{G \backslash E_{\alpha}}
$$

we see that

$$
\Phi_{X} \backslash E=\bigcup_{\alpha<\kappa}\left(\Phi_{X} \backslash \overline{G \backslash E_{\alpha}}\right)=\bigcup_{\alpha<\kappa} \overline{E_{\alpha}} .
$$

In the special case where $G$ is $\sigma$-compact, we have $E=\Phi_{X} \backslash G$, but this is not necessarily true in the general case, and this latter possibility makes the proof more complicated.

Let $C$ and $D$ be disjoint subsets of $T$. By (v), we have $C \cap U D=\emptyset$, and so, by Proposition 8.11(ii), $\bar{C} \cap \bar{D}=\emptyset$ in $\Phi_{X}$. Thus the natural map from $\beta G_{d}$ onto $\Phi_{X}$ has a restriction to $\beta T$ which is a homeomorphism onto $\bar{T}$.

We next claim that each $u \in T^{*} \cap E$ is right cancellable in $\left(\Phi_{X}, \square\right)$. To see this, take $x, y \in \Phi_{X}$ with $x \square u=y \square u$, and assume towards a contradiction that $x \neq y$. Let $K$ and $L$ be disjoint, compact neighbourhoods of $x$ and $y$, respectively, in $\Phi_{X}$. Then

$$
\begin{aligned}
& x \square u \in \bar{\bigcup}\left\{\left(E_{\beta} \cap K\right) t_{\alpha}: \beta<\alpha<\kappa\right\}, \\
& y \square u \in \bar{\bigcup}\left\{\left(E_{\beta} \cap L\right) t_{\alpha}: \beta<\alpha<\kappa\right\},
\end{aligned}
$$

where we are using here the fact that $u \in E$. By Proposition 8.11(i), there exists a symmetric neighbourhood $W$ of $e_{G}$ with $K \cap W L=\emptyset$; we may suppose that $W \subset U$. Since $x \square u=y \square u$, it follows from Proposition 8.11(ii) that there exist $\beta_{1}, \alpha_{1}, \beta_{2}, \alpha_{2}<\kappa$ with $\beta_{1}<\alpha_{1}$ and $\beta_{2}<\alpha_{2}$ such that

$$
\left(E_{\beta_{1}} \cap K\right) t_{\alpha_{1}} \cap W\left(E_{\beta_{2}} \cap L\right) t_{\alpha_{2}} \neq \emptyset .
$$


By clause (vi) in the construction, this can occur only if $\alpha_{1}=\alpha_{2}$. However, in this case, it follows that $K \cap W L \neq \emptyset$, a contradiction. Thus $u$ is indeed right cancellable, giving the claim. By Proposition 4.4(iii), we have $\|\mu\|=\|\mu \square u\|\left(\mu \in M\left(\Phi_{X}\right)\right)$ for each such $u$.

Define a map $\varphi: G \rightarrow \kappa$ by setting

$$
\varphi(s)=\min \left\{\alpha<\kappa: s \in E_{\alpha}\right\} .
$$

Each $\alpha<\kappa$ can be expressed in the form $\alpha=\beta_{\alpha}+n_{\alpha}$, where $\beta_{\alpha}$ is a limit ordinal or 0 and $n_{\alpha}<\omega$. For $s \in G$, we define

$$
\gamma(s) \equiv n_{\varphi(s)} \quad(\bmod 12)
$$

so that we have a map $\gamma: G \rightarrow \mathbb{Z}_{12}$.

Let $A=\left\{t_{\alpha}: \gamma\left(t_{\alpha}\right)=2\right\}$ and $B=\left\{t_{\alpha}: \gamma\left(t_{\alpha}\right)=8\right\}$, and choose $a \in A^{*} \cap E$ and $b \in B^{*} \cap E$, so that, by the above remark,

$$
\|\mu\|=\|\mu \square a\|=\|\mu \square b\| \quad\left(\mu \in M\left(\Phi_{X}\right)\right) .
$$

It follows from Proposition 5.4 that we can suppose that the element $a \in A$ has the extra property that, for each cardinal $\zeta<\kappa$, the set $A$ can be partioned into $\zeta$ pairwise-disjoint subsets $D_{\eta}$ such that $a$ does not belong to the closure of the union of strictly fewer than $\zeta$ sets in the partition $\left\{D_{\eta}: \eta<\zeta\right\}$ of $A$.

Set

$$
\begin{aligned}
X_{1} & =\{s \in G: \gamma(s) \in\{2,3\}\}, \\
X_{2} & =\{s \in G: \gamma(s) \in\{8,9\}\}, \\
Y_{1} & =\{s \in G: \gamma(s) \in\{0,1,2,3,4,5\}\}, \\
Y_{2} & =\{s \in G: \gamma(s) \in\{6,7,8,9,10,11\}\}, \\
Z & =\{s \in G: \gamma(s) \in\{5,6,7,8,9,10,11,0\}\} .
\end{aligned}
$$

so that $X_{1} \subset Y_{1}, X_{2} \subset Y_{2} \subset Z, Y_{1} \cup Y_{2}=G$, and $Y_{1} \cap Y_{2}=\emptyset$.

Let $u \in U$ and $s \in G$ with $\varphi(s)=\alpha$. Then $u s \in E_{\alpha+1}$ by the construction. Also, if $u s \in E_{\beta}$, then $s \in E_{\beta+1}$, and so we see that necessarily $\gamma(u s) \in\{\gamma(s)-1, \gamma(s), \gamma(s)+1\}$. Thus $X_{1} \cap U Z=\emptyset$, and So

$$
\overline{X_{1}} \cap \bar{Z}=\emptyset .
$$

Suppose that $s \in G$ with $\varphi(s)=\alpha$. Take $\delta$ with $\alpha<\delta<\kappa$. By (iv), above, st $t_{\delta} \in E_{\delta+1}$ and, by (vi), st $t_{\delta} \notin \bigcup\left\{E_{\beta}: \beta<\delta\right\}$, and so $\varphi\left(s t_{\delta}\right) \in\{\delta, \delta+1\}$. Thus $\gamma\left(s t_{\delta}\right) \in X_{1}$ whenever $\delta>\alpha$ and $t_{\delta} \in A$, and hence $u \square a \in \overline{X_{1}}$ when $u \in \Phi_{X}$. Now let $\mu \in M\left(\Phi_{X}\right)$. Then we have

$$
\operatorname{supp}(\mu \square a) \subset \overline{X_{1}} \text {. }
$$

Similarly, $\operatorname{supp}(\mu \square b) \subset \overline{X_{2}}$. 
Now fix $\beta<\kappa$ and take $s \in G$ with $\varphi(s)=\alpha$, where $\alpha>\beta+1$. Then $s t_{\beta} \in E_{\alpha+1}$ by (iv). Also, we see that $s t_{\beta} \notin E_{\delta}$ for any $\delta$ with $\delta+1<\alpha$ : this holds because the fact that $s t_{\beta} \in E_{\delta}$ implies that $s \in E_{\max \{\beta+1, \delta+1\}}$ by (iv). Thus it follows that $\varphi\left(s t_{\beta}\right) \in\{\alpha, \alpha+1\}$ whenever $\alpha$ is a limit ordinal or 0 and $\varphi\left(s t_{\beta}\right) \in\{\alpha-1, \alpha, \alpha+1\}$ otherwise. Thus

$$
\gamma\left(s t_{\beta}\right) \in\{\gamma(s)-1, \gamma(s), \gamma(s)+1\} .
$$

Hence, for each fixed $t \in T$ and each $u \in \overline{Y_{2}} \cap E$, we have $u \square t \in \bar{Z}$. This shows that

$$
\operatorname{supp}(\nu \square t) \subset \bar{Z} \quad\left(\nu \in M\left(\overline{Y_{2}} \cap E\right), t \in T\right) .
$$

We now fix $\mu \in M\left(\Phi_{X}\right)$ such that the map $L_{\mu}: \Phi_{X} \rightarrow M\left(\Phi_{X}\right)$ is $G$-continuous at both $a$ and $b$. We further set

$\mu_{1}=\mu\left|\left(\Phi_{X} \backslash E\right), \quad \mu_{2}=\mu\right| E, \quad \nu_{1}=\mu_{2}\left|\overline{Y_{1}}, \quad \nu_{2}=\mu_{2}\right|\left(\overline{Y_{2}} \backslash \overline{Y_{1}}\right)$, so that $\mu=\mu_{1}+\nu_{1}+\nu_{2}$, and hence

$$
\mu \square a=\lim _{t \rightarrow a} \mu \square t=\lim _{t \rightarrow a}\left(\mu_{1} \square t+\nu_{1} \square t+\nu_{2} \square t\right),
$$

where $t \in X_{1}$ in the limit, because $L_{\mu}$ is $G$-continuous at $a$.

We claim that in fact $\nu_{1}=\nu_{2}=0$, so that $\mu=\mu_{1}$. Indeed, since $\operatorname{supp}(\mu \square a) \subset \overline{X_{1}}$, it follows from (12.17), (12.16), and (12.14) that

$$
\mu \square a=\lim _{t \rightarrow a}\left(\mu_{1} \square t+\nu_{1} \square t\right) \mid \overline{X_{1}} .
$$

Thus $\|\mu\|=\|\mu \square a\| \leq\left\|\mu_{1}\right\|+\left\|\nu_{1}\right\|$. Similarly, $\|\mu\| \leq\left\|\mu_{1}\right\|+\left\|\nu_{2}\right\|$ because $L_{\mu}$ is $G$-continuous at $b$, and hence

$$
2\|\mu\| \leq 2\left\|\mu_{1}\right\|+\left\|\nu_{1}\right\|+\left\|\nu_{2}\right\| .
$$

But $2\|\mu\|=2\left\|\mu_{1}\right\|+2\left\|\nu_{1}\right\|+2\left\|\nu_{2}\right\|$, and so $\mu=\mu_{1}$, as claimed.

In the case where $G$ is $\sigma$-compact, so that $E=\Phi_{X} \backslash G$, we have already proved that $\mu \in M(G)$, giving the result (with $V=\{a, b\}$ ). Thus the remainder of the proof is necessary only in the case where $G$ is not $\sigma$-compact, and so $\kappa>\omega$; we now suppose that we are considering this case.

It follows from (12.13) that

$$
|\mu|\left(\bigcup_{\alpha<\kappa} \overline{E_{\alpha}}\right)=\|\mu\| .
$$

We now further assume towards a contradiction that $\mu \neq 0$. Since the measure $\mu$ is regular, it follows from (12.12) that there exists $\alpha<\kappa$ such that $|\mu|\left(\overline{E_{\alpha}}\right)>0$; we fix this index $\alpha$, and then we choose $\zeta$ 
to be the smallest cardinal number with the property there exists a Borel subset $H$ of $E_{\alpha}$ with a compact covering number $\zeta$ and such that $|\mu|(\bar{H})>0$. We observe that $\zeta$ is necessarily infinite and that $\zeta \leq|\alpha|<\kappa$.

Let $\left(C_{\eta}: \eta<\zeta\right)$ be a covering of $H$ by relatively compact subsets of $H$. For each $t_{\delta} \in A$, we define $\theta\left(t_{\delta}\right)<\zeta$ by the requirement that $t_{\delta} \in D_{\theta\left(t_{\delta}\right)}$. We then set

$$
S=\left\{s t_{\beta}: \beta>\delta+1, s \in H, \theta\left(t_{\beta}\right)<\min \left\{\eta<\zeta: s \in U^{2} C_{\eta}\right\}\right\} .
$$

We now claim that $\left(\Phi_{X} \square a\right) \cap \bar{S}=\emptyset$.

To see this, assume towards a contradiction that there exists an element $x \in\left(\Phi_{X} \square a\right) \cap \bar{S}$. Since $U \bar{S}$ is a neighbourhood of $x$, there exist $\beta<\kappa$ and $s \in E_{\beta}$ such that $s \square a \in U \bar{S}$. We fix such $s$ and $\beta$, and observe that there is a subset $A_{0}$ of $A$ such that

$$
A_{0} \subset\left\{t_{\gamma}: \gamma>\beta\right\}, \quad a \in \overline{A_{0}}, \quad \text { and } s A_{0} \subset U S .
$$

Hence we see from the definition of $S$ that, for each $t_{\gamma} \in A_{0}$, there exist $u \in U, \delta \in(\alpha+1, \kappa)$, and $\xi<\zeta$ such that $t_{\delta} \in A$ and $s t_{\gamma}=u v t_{\delta}$ for some $v \in C_{\xi}$ with

$$
\theta\left(t_{\delta}\right)<\min \left\{\eta<\zeta: v \in U^{2} C_{\eta}\right\} .
$$

Since $v \in E_{\alpha}$, we have $u v \in U E_{\alpha} \subset E_{\alpha+1}$, and so it follows from our construction of the set $T$ that $\delta=\gamma$, and hence that $s=u v \in U C_{\xi}$.

We now fix $\xi$, and repeat the preceding argument with any other element $t_{\gamma^{\prime}}$ in place of $t_{\gamma}$ to obtain the equation $s t_{\gamma^{\prime}}=u^{\prime} v^{\prime} t_{\delta^{\prime}}$ for some $u^{\prime} \in U$, some $v^{\prime} \in H$, and some $\delta^{\prime} \in(\alpha+1, \kappa)$, where $t_{\delta^{\prime}} \in A$ satisfies the condition that $\theta\left(t_{\delta^{\prime}}\right)<\min \left\{\eta<\zeta: v^{\prime} \in U^{2} C_{\eta}\right\}$. Again this implies that $s=u^{\prime} v^{\prime}$, and hence that $v^{\prime} \in U^{2} C_{\xi}$. So $\theta\left(t_{\gamma^{\prime}}\right)<\xi$, and hence we see that

$$
A_{0} \subset \bigcup_{\eta<\xi} D_{\eta}
$$

by our remarks on the partition $\left\{D_{\eta}: \eta<\zeta\right\}$ of $A$, this is a a contradiction of the assumption that $a \in \overline{A_{0}}$. Thus $\left(\Phi_{X} \square a\right) \cap \bar{S}=\emptyset$, as claimed.

Let $\rho=\mu \mid \bar{H}$, so that $|\rho|(\bar{H})>0$. Then

$$
\mu \square a=\lim _{t \rightarrow a} \mu \square t=\lim _{t \rightarrow a}(\rho \square t+(\mu-\rho) \square t) .
$$

But it is easy to see that $\operatorname{supp}\left(\lim _{t \rightarrow a}(\rho \square t)\right) \subset \bar{S}$, and so, by restricting the measures to the closed set $\Phi_{X} \square a$, we have

$$
\mu \square a=(\mu \square a)\left|\left(\Phi_{X} \square a\right)=\lim _{t \rightarrow a}((\mu-\rho) \square t)\right|\left(\Phi_{X} \square a\right),
$$


and this implies that

$$
\|\rho\|+\|\mu-\rho\|=\|\mu\|=\|\mu \square a\| \leq\|\mu-\rho\| .
$$

Hence $\rho=0$, a contradiction.

We have shown that the only element $\mu \in M\left(\Phi_{X}^{*}\right)$ such that the map $L_{\mu}: \Phi_{X} \rightarrow M\left(\Phi_{X}\right)$ is $G$-continuous at both $a$ and $b$ is $\mu=0$. Set $V=\{a, b\}$. Certainly each $\mu \in M(G)$ is such that $L_{\mu} \mid \Phi_{X}$ is continuous on $\Phi_{X}$. Further, $M\left(\Phi_{X}\right)=M(G) \ltimes M\left(\Phi_{X}^{*}\right)$, and so the result follows.

This concludes the proof of the theorem.

A similar result to the above has been proved by Filali and Salmi in $[45$, Theorem 14] in the special case where $G$ is $\sigma$-compact.

The result of Protasov mentioned above has been extended to the following by Protasov and Pym in [118]. Let $G$ be a locally compact, $\sigma$-compact, non-compact group, and set $X=L U C(G)$. Then there exists $v \in \Phi_{X}$ such that $u \in G$ whenever $u \in \Phi_{X}$ and $L_{u}: \Phi_{X}^{*} \rightarrow \Phi_{X}^{*}$ is continuous at $v$.

In our earlier memoir [21], we considered the topological centre of Beurling algebras $L^{1}(G, \omega)$; here, $\omega$ is a weight on the locally compact group $G$. We regret to say that the proof of Lemma 12.1 in $[\mathbf{2 1}]$ is incorrect, and that the result is false; we are grateful to Zhiguo $\mathrm{Hu}$ for pointing this out and for providing a counter-example to the statement. The lemma was used in the proof of Theorems 12.2 and 12.3 of [21]; the latter result states that $L^{1}(G, \omega)$ is strongly Arens irregular whenever $\omega$ is diagonally bounded on a dispersed subset of $G$.

However, as we remarked, the latter theorem can also be proved by a modification of the proof of [21, Theorem 11.9]. Further, we also noted that Neufang gave an independent proof of this theorem in [112]. 


\section{CHAPTER 13}

\section{Open problems}

We believe that the following questions are open.

(1) Let $S$ be a semigroup such that $\ell^{1}(S)$ has an identity $e_{A}$. Is $\left\|e_{A}\right\|_{1}$ necessarily rational? Is this true in the case where $\ell^{1}(S)$ is amenable?

(2) Let $S$ be a weakly cancellative semigroup.

Is $M\left(\overline{S_{[3]}^{*}}\right)$ a closed ideal in $M(\beta S)$ ? Is $M\left(\overline{\mathbb{N}_{[3]}^{*}}\right)$ a closed ideal in $M(\beta \mathbb{N})$ ?

(3) Let $S$ be a left-amenable semigroup. The subset $L(\beta S)$ of $\beta S$ was defined in Definition 9.11. Give an example where $L(\beta S)$ is not closed in $\beta S$. For example, is $L(\beta \mathbb{N})$ closed?

(4) Find an infinite semigroup $S$ such that

$$
\left(\mathfrak{Z}_{t}^{(\ell)}\left(A^{\prime \prime}\right) \cap \beta S\right) \subsetneq \mathfrak{Z}_{t}^{(\ell)}(\beta S),
$$

where $A=\ell^{1}(S)$.

(5) Let $S$ be a semigroup. When is $\left(\ell^{1}(\beta S), \square\right)$ semisimple?

Is this true whenever $S$ is cancellative? Is $\left(\ell^{1}(\beta \mathbb{N}), \square\right)$ semisimple? If these algebras are not semisimple, describe their radicals.

An example for which $\left(\ell^{1}(\beta S), \square\right)$ is not semisimple is given in Example 7.32.

(6) Let $S$ be a semigroup, and let $R_{M}$ be the radical of $(M(\beta S), \square)$. Is $R_{M}$ always nilpotent?

Is it always true that $R_{M}^{2}=0$ ? Is $\left(M\left(\beta \mathbb{F}_{2}\right), \square\right)$ semisimple? When are the radicals of the two algebras $(M(\beta S), \square)$ and $(M(\beta S), \diamond)$ equal as sets?

(7) The closure (in the topology of pointwise convergence on $\beta \mathbb{Z}$ ) of the set of continuous characters in $\Phi_{\beta \mathbb{Z}}$ consists of characters.

Are there any other characters? Is $\Psi_{\beta \mathbb{Z}}$, the space of characters on $\beta \mathbb{Z}$, connected? 
(8) Let $S$ be a semigroup such that $\ell^{1}(S)$ is amenable. Can it be that the amenability constant

$C_{S}$ of $\ell^{1}(S)$ belongs to the interval $(5,7)$ ? Give examples of semigroups $S$ such that

$$
C_{S} \notin\{1,5,7,9,11, \ldots\} \text {. }
$$

(9) Let $S$ be a semigroup, and set $X=W A P(S)$. Suppose that $\left(X^{\prime}, \square\right)$ is amenable. Does it follow that $S$ is finite?

(10) Is there a semigroup $S$ such that $M(\beta S)$ is weakly amenable, but $\ell^{1}(S)$ is not weakly amenable?

(11) Let $S$ be an infinite, (perhaps countable) cancellative semigroup. Show that there is no measure $\mu \in M\left(S^{*}\right)$ such that

$$
\max \{\|\mu \square a-\mu \diamond a\|,\|\mu \square b-\mu \diamond b\|\}<\|\mu\| .
$$

It is proved in Chapter 12 that this is true if the right-hand side of the above inequality be replaced by $\|\mu\| / 2$.

(12) Do there exist idempotents

$p$ and $q$ in $\beta \mathbb{N}$ (or $\beta S$, for some semigroups $S$ ) such that $\{p, q\}$ is determining for the left topological centre of $M(\beta \mathbb{N})$ ?

(13) For exactly which (non-abelian) groups $G$ is there an element $a \in G^{*}$ such that the singleton $\{a\}$ is determining for the left topological centre of $M(\beta G)$ ? Let $G=H \times F$, where $H$ is abelian and $F$ is finite. Then no singleton is determining for the left topological centre. Are these the only such groups?

(14) For which semigroups $S$ is

$(M(\beta S), \square)$ strongly Arens irregular? 


\section{Bibliography}

[1] P. D. Adams, Algebraic topics in the Stone-Čech compactifications of semigroups, Thesis, University of Hull, 2001.

[2] R. Arens, Operations induced in function classes, Monatsh Math., 55 (1951), $1-19$.

[3] R. Arens, The adjoint of a bilinear operation, Proc. American Math. Soc., 2 (1951), 839-848.

[4] J. W. Baker and A. Rejali, On the Arens regularity of weighted convolution algebras, J. London Math. Soc. (2), 40 (1989), 535-546.

[5] M. L. Bami, The topological centers of $L U C(S)^{*}$ and $M_{a}(S)^{* *}$ of certain foundation semigroups, Glasgow Maths. Journal, 42 (2000), 335-343.

[6] B. A. Barnes and J. Duncan, The Banach algebra $\ell^{1}(S)$, J. Functional Analysis, 18 (1975), 96-113.

[7] M. Beiglböck, V. Bergelson, N. Hindman, and D. Strauss, Multiplicative structures in additively large sets, J. Combinatorial Theory (Series A), 113 (2006), 1219-1242.

[8] C. Berg and J. P. R. Christensen, Positive definite functions on abelian semigroups, Math. Annalen, 223 (1976), 253-272.

[9] C. Berg, J. P. R. Christensen, and P. Ressel, Harmonic analysis on semigroups, Graduate Texts in Mathematics, 100, Springer-Verlag, New York, 1984.

[10] J. F. Berglund, H. D. Junghenn, and P. Milnes, Analysis on semigroups; function spaces, compactifications, representations, Canadian Math. Soc. Series of Monographs and Advanced Texts, John Wiley, New York, 1989.

[11] T. D. Blackmore, Weak amenability of discrete semigroup algebras, Semigroup Forum, 55 (1997), 196-205.

[12] D. P. Blecher, Are operator algebras Banach algebras?, Contemporary Mathematics; Banach algebras and their applications, 363 (2003), 53-58.

[13] S. Burns, The existence of disjoint smallest ideals in the two natural products on $\beta S$, Semigroup Forum, 63 (2001), 191-201.

[14] R. J. Butcher, Thesis, University of Sheffield, 1975.

[15] T. J. Carlson, N. Hindman, J. McLeod, and D. Strauss, Almost disjoint large subsets of semigroups, Topology and its applications, 158 (2008), 433-444. 
[16] P. Civin and B. Yood, The second conjugate space of a Banach algebra as an algebra, Pacific J. Math., 11 (1961), 847-870.

[17] A. H. Clifford and G. B. Preston, The algebraic theory of semigroups, Volumes I and II, American Math. Soc., Providence, Rhode Island, 1961 and 1967.

[18] W. N. Comfort and S. Negrepontis, The theory of ultrafilters, SpringerVerlag, Berlin, Heidelberg, and New York, 1974.

[19] H. G. Dales, Banach algebras and automatic continuity, London Math. Society Monographs, Volume 24, Clarendon Press, Oxford, 2000.

[20] H. G. Dales and H. Dedania, Weighted convolution algebras on subsemigroups of the real line, submitted.

[21] H. G. Dales and A. T.-M. Lau, The second duals of Beurling algebras, Memoirs American Math. Soc., 177 (2005), 1-191.

[22] H. G. Dales and W. H. Woodin, An introduction to independence for analysts, London Mathematical Society Lecture Notes 115, Cambridge University Press, 1987.

[23] H. G. Dales, F. Ghahramani, and A. Ya. Helemskii, The amenability of measure algebras, J. London Math. Soc. (2), 66 (2002), 213-226.

[24] H. G. Dales, A. T.-M. Lau, and D. Strauss, The second duals of measure algebras, preprint.

[25] H. G. Dales, N. J. Laustsen, and C. J. Read, A properly infinite Banach *-algebra with a non-zero, bounded trace, Studia Mathematica, 155 (2003), $107-129$.

[26] H. G. Dales, R. J. Loy, and Y. Zhang, Approximate amenability for Banach sequence algebras, Studia Mathematica, 177 (2006), 81-96.

[27] H. G. Dales, A. Rodrigues-Palacios, and M. V. Velasco, The second transpose of a derivation, J. London Math. Soc. (2), 64 (2001), 707-721.

[28] M. Daws, Dual Banach algebras: representations and injectivity, Studia Mathematica, 178 (2007), 231-275.

[29] M. M. Day, Amenable semigroups, Illinois J. Math., 1 (1957), 509-544.

[30] M. Despić and F. Ghahramani, Weak amenability of group algebras of locally compact groups, Canadian Math. Bulletin (2), 37 (1994), 165-167.

[31] J. Duncan and S. A. R. Hoseiniun, The second dual of a Banach algebra, Proc. Royal Soc. Edinburgh, Section A, 84 (1979), 309-325.

[32] J. Duncan and I. Namioka, Amenability of inverse semigroups and their semigroup algebras, Proc. Royal Soc. Edinburgh, Section A, 80 (1978), 309321.

[33] J. Duncan and A. L. T. Paterson, Amenability for discrete convolution semigroup algebras, Math. Scandinavica, 66 (1990), 141-146. 
[34] C. F. Dunkl and D. E. Ramirez, Weakly almost periodic functions on the Fourier algebra, Trans. American Math. Soc., 185 (1973), 501-514.

[35] C. F. Dunkl and D. E. Ramirez, Representations of commutative semitopological semigroups, Lecture Notes in Mathematics 435 (1975), SpringerVerlag, Berlin-New York.

[36] M. Eshaghi Gordji and M. Filali, Weak amenability of the second dual of a Banach algebra, Studia Mathematica, 182 (2007), 205-213.

[37] G. H. Esslamzadeh, Banach algebra structure and amenability of a class of matrix algebras with applications, J. Functional Analysis, 161 (1999), $364-383$.

[38] G. H. Esslamzadeh, Duals and topological center of a class of matrix algebras with applications, Proc. American Math. Soc., 128 (2000), 3493-3503.

[39] P. Eymard, L'algèbre de Fourier d'un groupe localement compact, Bull. Société Mathématique de France, 92 (1964), 181-236.

[40] L. R. Fairchild, Extreme invariant means without minimal support, Trans. American Math. Soc., 172 (1972), 83-93.

[41] S. Ferri and M. Neufang, On the topological centre of the algebra $\operatorname{LUC}(\mathcal{G})^{*}$ for general topological groups, J. Functional Analysis, 244 (2007), 154-171.

[42] S. Ferri and D. Strauss, Ideals, idempotents and right cancellable elements in the uniform compactification, Semigroup Forum, 63 (2001), 449-456.

[43] S. Ferri and D. Strauss, A note on the $W A P$-compactification and the $L U C$ compactification of a topological group, Semigroup Forum, 69 (2004), 87101.

[44] M. Filali, Finite-dimensional right ideals in some algebras associated with a locally compact group, Proc. American Math. Soc., 127 (1999), 1729-1734.

[45] M. Filali and P. Salmi, Slowly oscillating functions in semigroup compactifications and convolution algebras, J. Functional Analysis, 250 (2007), 144166.

[46] M. Filali and A. I. Singh, Recent developments on Arens regularity and ideal structures of the second dual of a group algebra (Tartu, 1999), Mathematical Studies (Tartu), 1 (2001), 95-124.

[47] B. Forrest, Weak amenability and the second dual of the Fourier algebra, Proc. American Math. Soc., 125 (1997), 2373-2378.

[48] B. Forrest and V. Runde, Amenability and weak amenability of the Fourier algebra, Math. Zeitschrift, 250 (2005), 731-744.

[49] F. Ghahramani and J. Laali, Amenability and topological centres of the second duals of Banach algebras, Bull. Australian Math. Soc., 65 (2002), 191-197.

[50] F. Ghahramani and A. T.-M. Lau, Multipliers and ideals in second conjugate algebras related to locally compact groups, J. Functional Analysis, 132 (1995), 170-191. 
[51] F. Ghahramani and R. J. Loy, Generalized notions of amenability, J. Functional Analysis, 208 (2004), 229-260.

[52] F. Ghahramani and J. P. McClure, Module homomorphisms of the dual modules of convolution Banach algebras, Canadian Math. Bulletin, 35 (1992), 180-185.

[53] F. Ghahramani, A. T.-M. Lau, and V. Losert, Isometric isomorphisms between Banach algebras related to locally compact groups, Trans. American Math. Soc., 321 (1990), 273-283.

[54] F. Ghahramani, R. J. Loy, and G. A. Willis, Amenability and weak amenability of second conjugate Banach algebras, Proc. American Math. Soc., 124 (1996), 1489-1496.

[55] F. Ghahramani, R. J. Loy, and Y. Zhang, Generalized notions of amenability, II, J. Functional Analysis, 254 (2008), 1776-1810.

[56] M. Ghandehari, H. Hatani, and N. Spronk, Amenability constants for semilattice algebras, Semigroup Forum, 79 (2009), 279-293.

[57] L. Gillman and N. Jerison, Rings of continuous functions, van Nostrand, Princeton, New Jersey, 1960.

[58] I. Glicksberg, Uniform boundedness for groups, Candian J. Mathematics, 14 (1962), 269-276.

[59] F. Gourdeau, Amenability and the second dual of Banach algebras, Studia Mathematica, 125 (1997), 75-81.

[60] E. E. Granirer, On amenable semigroups with a finite dimensional set of invariant means, I and II, Illinois J. Math., 7 (1963), 32-48 and 49-58.

[61] E. E. Granirer, Extremely amenable semigroups, Math. Scandinavica, 17 (1965), 177-197.

[62] E. E. Granirer, Exposed points of convex sets and weak sequential convergence, Memoirs American Math. Soc., 123 (1972), 1-180.

[63] E. E. Granirer, The radical of $L^{\infty}(G)^{*}$, Proc. American Math. Soc., 41 (1973), 321-324.

[64] M. Grosser, Bidualräume und Vervollständigungen von Banach-moduln, Lecture Notes in Mathematics, 717 (1979), Springer-Verlag, Berlin.

[65] M. Grosser and V. Losert, The norm-strict bidual of a Banach algebra and the dual of $C_{u}(G)$, Manuscripta Mathematica, 45 (1984), 127-146.

[66] N. Grønbæk, A characterisation of weakly amenable Banach algebras, Studia Mathematica, 94 (1989), 149-162.

[67] N. Grønbæk, Amenability of weighted discrete semigroup convolution algebras, Proc. Royal Soc. Edinburgh, Section A, 110 (1988), 351-360.

[68] N. Grønbæk, Amenability of discrete convolution algebras, the commutative case, Pacific J. Mathematics, 143 (1990), 243-249. 
[69] A. Grothendieck, Critères de compacité dans les espaces fonctionelles généraux, American J. Mathematics, 74 (1952), 168-186.

[70] U. Haagerup, All nuclear $C^{*}$-algebras are amenable, Inventiones Math., 74 (1983), 305-319.

[71] S. Hartman and C. Ryll-Nardzewski, Almost periodic extensions of functions II, Colloquium Math., 15 (1966), 79-86.

[72] E. Hewitt and K. A. Ross, Abstract harmonic analysis, Volume I (Second edition), Springer-Verlag, Berlin, 1979.

[73] E. Hewitt and H. S. Zuckerman, The $\ell_{1}$-algebra of a commutative semigroup, Trans. American Math. Soc., 83 (1956), 70-97.

[74] N. Hindman, Minimal ideals and cancellation in $\beta \mathbb{N}$, Semigroup Forum, 25 (1982), 291-310.

[75] N. Hindman, The ideal structure of the space of $\kappa$-uniform ultrafilters on a discrete semigroup, Rocky Mountain J. Mathematics, 16 (1986), 685-701.

[76] N. Hindman and J. S. Pym, Free groups and semigroups in $\beta \mathbb{N}$, Semigroup Forum, 30 (1984), 177-193.

[77] N. Hindman and D. Strauss, Prime properties of the smallest ideal of $\beta \mathbb{N}$, Semigroup Forum, 52 (1996), 357-364.

[78] N. Hindman and D. Strauss, Algebra in the Stone-Čech compactification, Expositions in Mathematics 27, de Gruyter, Berlin, 1998.

[79] N. Hindman and D. Strauss, Characterizations of simplicity and cancellativity in $\beta S$, Semigroup Forum, 75 (2007), 70-78.

[80] M. Hochster, Subsemigroups of amenable groups, Proc. American Math. Soc., 21(1969), 363-364.

[81] J. M. Howie, Fundamentals of semigroup theory, London Math. Society Monographs, Volume 12, Clarendon Press, Oxford, 1995.

[82] N. Işik, J. Pym, and A. Ülger, The second dual of the group algebra of a compact group, J. London Math. Soc. (2), 35 (1987), 135-158.

[83] B. E. Johnson, Cohomology in Banach algebras, Memoirs American Math. Soc., 127 (1972), 1-96.

[84] B. E. Johnson, Approximate diagonals and cohomology of certain annihilator Banach algebras, American J. Mathematics, 94 (1972), 685-698.

[85] B. E. Johnson, Weak amenability of group algebras, Bull. London Math. Soc., 23 (1991), 281-284.

[86] B. E. Johnson, Non-amenability of the Fourier algebra of a compact group, J. London Math. Soc. (2), 50 (1994), 361-374.

[87] M. Klawe, On the dimension of left invariant means and left thick subsets, Trans. American Math. Soc., 231 (1977), 507-518. 
[88] M. Koçak and D. Strauss, Near ultrafilters and compactifications, Semigroup Forum, 55 (1997), 94-109.

[89] A. T.-M. Lau, Topological semigroups with invariant means in the convex hull of multiplicative means, Trans. American Math. Soc., 148 (1970), 69-84.

[90] A. T.-M. Lau, Operators which commute with convolutions on subspaces of $L_{\infty}(G)$, Colloquium Math., 39 (1978), 357-359.

[91] A. T.-M. Lau, Analysis on a class of Banach algebras with applications to harmonic analysis on locally compact groups and semigroups, Fundamenta Mathematica, 118 (1983), 161-175.

[92] A. T.-M. Lau, Continuity of Arens multiplication on the dual space of bounded uniformly continuous functions on locally compact groups and topological semigroups, Math. Proc. Cambridge Philosophical Soc., 99 (1986), 273-283.

[93] A. T.-M. Lau, Amenability of semigroups, The analytic and topological theory of semigroups (ed. K. H. Hoffmann, J. D. Lawson, and J. S. Pym), de Gruyter, Berlin (1990), 313-334.

[94] A. T.-M. Lau, Fourier and Fourier-Stieltjes algebras of a locally compact group and amenability, Topological vector spaces, algebras and related areas, (ed. A. T.-M. Lau and I. Tweddle), Pitman Research Notes in Mathematics Series, 316, New York (1994).

[95] A. T.-M. Lau and V. Losert, On the second conjugate algebra of $L_{1}(G)$ of a locally compact group, J. London Math. Soc. (2), 37 (1988), 464-470.

[96] A. T.-M. Lau and R. J. Loy, Weak amenability of Banach algebras on locally compact groups, J. Functional Analysis, 145 (1997), 175-204.

[97] A. T.-M. Lau and A. L. T. Paterson, The exact cardinality of the set of topological left invariant means on an amenable locally compact group, Proc. American Math. Soc., 98 (1986), 75-80.

[98] A. T.-M. Lau and J. S. Pym, The topological centre of a compactification of a locally compact group, Math. Zeitschrift, 219 (1995), 567-569.

[99] A. T.-M. Lau and A. Ülger, Topological centers of certain dual algebras, Trans. American Math. Soc., 346 (1996), 1191-1212.

[100] A. T.-M. Lau, R. J. Loy, and G. A. Willis, Amenability of Banach and $C^{*}$-algebras on locally compact groups, Studia Mathematica, 119 (1996), $161-178$.

[101] A. T.-M. Lau, A. R. Medghalchi, and J. S. Pym, On the spectrum of $L^{\infty}(G)$, J. London Math. Soc. (2), 48 (1993), 152-166.

[102] E. S. Ljapin, Semigroups, Translations of Mathematical Monographs, Volume 3, American Mathematical Society, Providence, Rhode Island, 1974.

[103] V. Losert, Weakly compact multipliers on group algebras, J. Functional Analysis, 213 (2004), 466-472. 
[104] N. Macri, The continuity of Arens' product on the Stone-Čech compactification of semigroups, Trans. American Math. Soc., 191 (1974), 185-193.

[105] P. Milnes, Uniformity and uniformly continuous functions for locally compact groups, Proc. American Math. Soc., 109 (1990), 567-570.

[106] T. Mitchell, Fixed points and multiplicative left invariant means, Trans. American Math. Soc., 122 (1966), 195-202.

[107] T. Mitchell, Topological semigroups and fixed points, Illinois J. Math., 14 (1970), 630-641.

[108] W. D. Munn, On semigroup algebras, Proc. Cambridge Philosophical Soc., 51 (1955), 1-15.

[109] I. Namioka, On certain actions of semi-groups on L-spaces, Studia Mathematica, 29 (1967), 63-77.

[110] M. Neufang, A unified approach to the topological centre problem for certain Banach algebras arising in harmonic analysis, Archiv der Mathematik (Basel), 82 (2004), 164-171.

[111] M. Neufang, On a conjecture by Ghahramani-Lau and related problems concerning topological centres, J. Functional Analysis, 224 (2005), 217-229.

[112] M. Neufang, On the topological centre problem for weighted convolution algebras and semigroup compactifications, Proc. American Math. Soc., 136 (2008), 1831-1839.

[113] T. W. Palmer, Banach algebras and the general theory of ${ }^{\star}$-algebras, Volume I, Algebras and Banach algebras, Cambridge University Press, 1994.

[114] T. W. Palmer, Banach algebras and the general theory of ${ }^{\star}$-algebras, Volume II, *-algebras, Cambridge University Press, 2001.

[115] D. J. Parsons, The centre of the second dual of a commutative semigroup algebra, Math. Proc. Cambridge Philosophical Soc., 95 (1984), 71-92.

[116] A. L. T. Paterson, Amenability, Mathematical Surveys and Monographs, 29, American Math. Soc., Providence, Rhode Island, 1988.

[117] I. V. Protasov, The topological center of the semigroup of free ultrafilters, Mat. Zametki, 63 (1998), 437-441; translation in Math. Notes, 63 (1998), 384-387.

[118] I. V. Protasov and J. S. Pym, Continuity of multiplication in the largest compactification of a locally compact group, Bull. London Math. Soc., 33 (2001), 279-282.

[119] J. S. Pym, The convolution of functionals on spaces of bounded functions, Proc. London Math. Soc. (3), 15 (1965), 84-104.

[120] J. S. Pym, Semigroup structure in Stone-Čech compactifications, J. London Math. Soc. (2), 36 (1987), 421-428.

[121] D. Rees, On semi-groups, Proc. Cambridge Philosophical Soc., 36 (1940), $387-400$. 
[122] D. Rees, Note on semi-groups, Proc. Cambridge Philosophical Soc., 37 (1941), 434-435.

[123] W. Rudin Real and complex analysis, McGraw-Hill, New York, 1966.

[124] V. Runde, Amenability for dual Banach algebras, Studia Mathematica, 148 (2001), 47-66.

[125] V. Runde, Lectures on amenability, Lecture Notes in Mathematics, 1774 (2002), Springer-Verlag, Berlin.

[126] V. Runde, The amenability constant of the Fourier algebra, Proc. American Math. Soc., 134 (2006), 1473-1481.

[127] V. Runde, Cohen-Horst type idempotent theorems for representations on Banach spaces and applications to Figà-Talamanca-Herz algebras, Journal of Mathematical Analysis and its Applications, 329 (2007), 736-751.

[128] V. Runde and N. Spronk, Operator amenability of Fourier-Stieltjes algebras, Math. Proc. Cambridge Philosophical Soc., 136 (2004), 675-686.

[129] V. Runde and N. Spronk, Operator amenability of Fourier-Stieltjes algebras, II, Bull. London Math. Soc., 39 (2007), 194-202.

[130] S. Shelah, Proper forcing, Lecture Notes in Mathematics 940, SpringerVerlag, Berlin, 1982.

[131] R. Stokke, Approximate diagonals and Følner conditions for amenable groups and semigroups, Studia Mathematica, 164 (2004), 139-159.

[132] E. K. van Douwen, The maximal totally bounded group topology on $G$ and the biggest minimal $G$-space for abelian groups $G$, Topology Appl., 34 (1990), 69-91.

[133] C. O. Wilde and K. G. Witz, Invariant means and the Stone-Čech compactification, Pacific J. Math., 21 (1967), 577-586.

[134] E. Wimmers, The Shelah P-point independence theorem, Israel J. Mathematics, 43 (1982), 28-48.

[135] K. G. Witz, Applications of a compactification for bounded operator semigroups, Illinois J. Math., 26 (1982), 143-154.

[136] Z. Yang, On the set of invariant means, J. London Math. Soc. (2), 39 (1988), 317-330.

[137] N. J. Young, Separate continuity and multilinear operations, Proc. London Math. Soc. (3), 26 (1973), 289-319.

[138] N. J. Young, Semigroup algebras having regular multiplication, Studia Mathematica, 47 (1973), 191-196.

[139] N. J. Young, The irregularity of multiplication in group algebras, Quarterly J. Math. Oxford (2), 24 (1973), 59-62.

[140] Y. Zhang, Weak amenability of a class of Banach algebras, Canadian Math. Bulletin, 44 (2001), 504-508. 


\section{Index}

algebra, 2

Cuntz, 24

Fourier, 132

group, 10, 113, 131

measure, 10, 116, 131

Munn, 24, 25, 61

opposite, 2

radical, 181

Rees semigroup, 61

semigroup, 53

amenable, 131, 133, 137

weakly amenable, 133-134

semisimple, 3, 181

almost left disjoint, 45, 46, 50, 80

almost periodic, 31, 110, 114

amenability constant, 14, 131-132, 140,181

amenable Banach algebra, 13, 29

$A(G), 132$

$\ell^{1}(S), 133,137$

$L^{1}(G), 131$

$\ell^{1}(\beta S), 152$

$M(\beta S), 149,151$

amenable group, 119, 131

amenable semigroup, 123

annihilator, 5

approximate identity, 8,55

bounded, 8, 99

approximately amenable, 19, 131, 135

Arens product, 26

first, second, 26

Arens regular, 29, 31, 75, 157

Banach algebra, 7

amenable 13

$C$-amenable, 14, 131, 140

dual, 28, 59, 95

weakly amenable, 13, 152-154
Banach $A$-bimodule, 13

Banach space, 5

dual, 13

Borel measure, 6, 89

Borel set, 89

cancellable, 42

left, right, 42

cancellative semigroup, 42

left, right, 42

weakly, 42

left, right, 42

nearly, 43

left, right, 43

cardinal, 2

regular, 2

centre, 2

character, 3, 48, 106, 181

augmentation, 48, 54, 89, 110

discrete augmentation, 110 ,

115-116

semi-, 48, 106

continuous, 104

compact covering number, 175

compactifiction

Bohr, 111, 115

Stone-Čech, 67, 71

commutator subgroup, 49

Continuum Hypothesis, 2

Cuntz algebra, 24

derivation, 4, 56

approximately inner, 19

inner, 4

point, 4, 153

determinant, 4

determining for the topological centre, 157-158, 161, 169, 182

diagonal, 5, 142-143 
bounded approximate, 14

virtual, 14

diagonally bounded, 180

dual Banach algebra, 28, 59

essential, 8

factors, 3

finite product, 33,74

distinct, 34

finite sum, 34

Fourier algebra , 132

Fourier transform, 54

full subalgebra, 3

Gel'fand transform, 3

group, 33

amenable, 119, 131

free, 52

maximally almost periodic, 48, 115

topological, 47

group algebra, 10,131

amenable, 131

weakly amenable, 131

growth, 7, 67, 117

$G$-continuous, 177

ideal, 35

finite group, 35, 120

left, right, 35

maximal, 35

right, 47

minimal, 35, 47

minimum, 35, 47

nilpotent, 101

prime, 35, 104

idempotent, 2, 34, 182

minimal, 2, 35, 47, 121

left, 37

primitive, 35

right maximal, 35, 47, 74, 77

index, 57

introverted, 31, 110, 118

$C^{*}$-subalgebra, 90, 109-110, 152, 155,175

left-, right-, 90, 113

left-, right-, 31

length, 52

locally compact group, 10 almost abelian, 133

matrix units, 4, 41, 65

matrix, identity, 40

mean, 119

invariant, 119

left-, right-, 119

measure, 6

Borel, 6

continuous, 7

discrete, 7

measure algebra, 10, 116, 131

amenable, 132

Munn algebra, 24, 25, 61

nilpotent, 3, 101-103, 181

operator $C$-amenability, 135

ordinal, 2

period, 57

principal series, 36

projective tensor product, 5,9

$P$-point, 69

quasi-invertible, 3

quasi-nilpotent, 3, 102-103

quasi-order, 35

radical, 3, 101

Rees semigroup, 39, 52, 136, 139, 142

Rees semigroup algebra, 61, 78, 96

sandwich matrix, 24, 40

regular, 40

semi-character, 48

semi-direct product, 2

semigroup, 33

amenable, 119, 123

extremely left-, 123

left-, 119, 124, 127, 181

right-, 119

cancellative, 42

completely o-simple, 37

completely regular, 134

direct product, 43

direct sum, 43

dual, 49, 104

free, 8,52

o-simple, 37 
left zero, 49, 72

maximally almost periodic, 48, 126

opposite, 33

quotient, 36

Rees matrix, 39, 52, 61, 78, 96, 136-137,

regular, 39, 52, 139

regular, 34, 73

right topological, 116

right zero, 49, 60, 72, 107, 125,

134,150

semi-topological, compact, 110

simple, 36

topological, 47, 111

left, right, 47

trivial, 49, 155

weakly cancellative, 42, 59, 95, 181

zero, 34

semilattice, 150

semisimple, 3

semi-topological semigroup, 47

spectrum, 3, 57, 92

square-free, 130

strongly Arens irregular, 29, 75, 76,

97, 107-108, 158, 173, 182

left, right $29,75,76$

support, 6

theorem

Butcher, 158

Civin and Yood, 102, 118

Cohen's factorization, 8

Dales, Ghahramani, and

Helemskii, 132

Duncan and Paterson, 140

Esslamzadeh, 157

Forrest and Runde, 133

Ghahramani and Loy, 19

Ghahramani and McClure, 118

Granirer, 114

Grønbæk, 134

Işik, Pym, and Ülger, 118

Johnson, 15, 18, 131-133

Lau and Losert, 118

Neufang, 108, 118, 159, 180

Protasov, 175

Protasov and Pym, 180

Rees, 41

Runde, 132
Shelah, 69

Stokke, 131, 140

structure, 36, 37, 47, 137

Szemerédi, 130

Young, 118

topological centre, 28, 32, 117, 157

determining for, 157-158, 161, 169,182

left, right, 29, 75

topological group, 47

topological semigroup, 47, 110

compact, right, 47, 72, 91, 109

left, right, 47

translation maps, 56

ultrafilter, 56, 67, 73

$\kappa$-uniform, 68, 82, 97

uniform algebra, 6

uniform norm, 6

uniformly continuous, left, right, 114

upper Banach density, 129

upper density, 129

weakly almost periodic, 31, 110, 114

weakly amenable Banach algebra,

13, 152-154

$A(G), 133$

$\ell^{1}(S), 133-134,153$

$L^{1}(G), 131$

$L^{1}(G)^{\prime \prime}, 155$

$M\left(S^{*}\right), 152$

$M(\beta S), 153-154$

$\mathrm{ZFC}, \mathrm{ZFC}+\mathrm{CH}, 2$ 



\section{Index of Symbols}

$A^{\prime}, A^{\prime \prime}, 13$
$A^{\mathrm{op}}, A^{\#}, 2$
$A=B \ltimes I, 2$
$\left(A \widehat{\otimes} B,\|\cdot\|_{\pi}\right), 9$
$a d_{x}, 4$
$A_{D}, 15,59$
$A(G), 131-133$
$A_{p}(G), 132$
$A M(A), 14-16,131$
$A M\left(A_{D}\right), 16$
$A M(A(G)), 132-133$
$A M\left(A_{p}(G)\right), 133$
$A M\left(L^{1}(G)\right), 131$
$A P(A), 31$
$A P(G), 114$
$A P(S), 110$
$A\left(\Phi_{S}\right), 54$
$A_{j, r}, 87$
$\aleph_{0}, \aleph_{1}, 2$
$\mathcal{B}(E, F), \mathcal{B}(E), 5$
$b G, 114-116$
$b S, 111$
$\beta S, 68,71$
$\beta \Omega, 67$
$c_{0}, c_{0}(S), 5$
$C(\Omega), C B(\Omega), C_{0}(\Omega), C_{00}(\Omega)$
$C_{\mathbb{R}}(\Omega), 6$
$C_{S}, 140-142,145,147,181$
$\chi_{T}, 2$
$D, 34,59,141$
$D_{S}, 80-81$

$\bar{d}(U), d^{*}(U), 129$

$\operatorname{det} a, 4$

$\mathbb{D}, 2$

$\delta_{s}, 2$

$\Delta, 129$

$e_{A}, 2$

$\left\|e_{A}\right\|_{1}, 53,63,138,145-146,181$

$e_{S}, 33$

$E_{[r]}, E^{\prime}, E^{\prime \prime}, 5$

$E_{i j}, 4$

$E(S), 34,140$

$E(\beta S), 72$

$\overline{E(K(\beta \mathbb{N}))}, 165$

$E_{S}, 140,145$

$F^{\circ}, 5$

$[F], 44$

$F P\left\langle\left(s_{n}\right)\right\rangle, F S\left\langle\left(s_{n}\right)\right\rangle, 34$

$\widehat{f}, 54$

$\mathbb{F}_{2}, 52,79,102,120,174$

$\Phi_{A}, 3$

$\Phi_{S}, 48,54,104$

$\Phi_{X}, 90,109$

$\Phi_{X}^{*}, 109$

$\left(\Phi_{X}^{*}\right)_{[2]}, 109$

$\Phi_{L U C(G)}, \Phi_{R U C(G)}, 114$

$\Phi_{A P(G)}, \Phi_{W A P(G)}, 114$

$\Phi_{\beta \mathbb{Z}}, 181$

$\varphi_{S}, 54$

$\varphi^{\prime \prime}, 27$

$\varphi_{S}^{\prime \prime}, 89$

$\varphi \cdot \psi, 48$

$\widetilde{\varphi}_{X, G}, 114$ 
$\widetilde{\varphi}_{X, S}, 110$

$\widetilde{\varphi}_{S}, 110,153$

$\mathcal{G}, 3$

$\gamma_{X}, 91,109$

$\mathbb{H}, 85$

$I_{n}, 4$

$I_{G}(n), 40$

$I(K), 7$

$\mathbb{I}, 2$

$\mathfrak{I}(A), 2$

$K(S), 36,134,137$

$K(V), 47$

$K(\beta S, \square), 72,129$

$K\left(M_{1}(\beta S)\right), 122$

$K(\mathfrak{M}(S)), 120,122$

$\operatorname{lin} S, 4$

$L^{1}(G), 10,113$

$L^{1}(G)^{\prime \prime}, 155$

$L^{1}(G, \omega), 180$

$L_{s}, 41$

$L_{v}, 56$

$L_{\mu}, 117$

$\operatorname{LUC}(G), 114$

$L(\beta S), 124,128-129,131,181$

$L(\beta \mathbb{N}), 131$

$L_{0}(\beta \mathbb{N}), 129$

$L O(\lambda), 110,114$

$\mathfrak{L}(S), 119$

$\mathfrak{L}_{0}(\mathbb{N}), 129$

$\ell_{u}, 112$

$\ell_{t}, 113$

$\mathcal{L}(E, F), \mathcal{L}(E), 4$

$\ell^{\infty}, \ell^{p}, \ell_{n}^{p}, \ell^{1}(S), 5$

$\ell^{\infty}(S), 5,67$

$\ell^{1}(S) \widehat{\otimes} \ell^{1}(T), 5$

$\left(\ell^{1}(S), \star\right), \ell^{1}(G), 53$

$\ell^{1}(\beta S), 95$

$\ell^{1}\left(\mathbb{F}_{2}\right), 102$
$\Lambda(\beta S), 75$

$m_{A}, 2$

$m_{S}, 33,47$

$M_{\mathbb{R}}(\Omega), M(\Omega)^{+}, M(\Omega), 6$

$M(G), 10,116$

$M_{a c}(G), M_{c}(G), 10$

$M(V), 56$

$M\left(S^{*}\right), 7,152$

$M(\beta S), 68,89,95,149,153$

$M\left(\overline{S_{[n]}^{*}}\right), 93$

$M\left(U_{\kappa}(S)\right), 98$

$M(\overline{K(\beta \mathbb{N})}), 131$

$M\left(\Phi_{X}\right), 115$

$M(b G), 116$

$M_{0}(\beta S), M_{1}(\beta S), 121$

$\mathcal{M}(A, P, m, n), \mathcal{M}(A, P, n), 24$

$\mathcal{M}^{o}(\mathbb{C}, P, n), 62,138,142$

$\mathcal{M}^{\circ}(G, P, m, n), 40,61,78,81,96$,

136

$\mathcal{M}^{o}(G, P, n), 65,137$

$\mathcal{M}\left(T, P_{G}, m, n\right), 39$

$\mathcal{M}^{o}\left(T, P_{G}, n\right), \mathcal{M}^{o}(P, m, n), 40$

$\mathcal{M}^{o}\left(\ell^{1}(G), P, m, n\right), 62,96,136$

$\mathcal{M}^{o}\left(\ell^{1}(G), P, n\right), 62$

$\mathcal{M}^{o}\left(\beta G, P_{G}, m, n\right), 78$

$\mathcal{M}^{o}\left(G^{*}, P_{G}, m, n\right), 78$

$\mathcal{M}(M(\beta G), P, m, n), 97$

$\mathbb{M}_{m, n}(S), \mathbb{M}_{n}(S), \mathbb{M}_{n}(A), \mathbb{M}_{m, n}, 4$

$\mathbb{M}_{n}(\mathbb{N}), 51$

$\mathfrak{M}(S), 119$

$|\mu|, 6$

$\mu_{d}, \mu_{c}, 7$

$\mu \square \nu, \mu \diamond \nu, 89$

$N_{A}, 3,8$

$N(P), 61$

$N\left(P_{G}\right), 41$

$\mathbb{N}, \mathbb{N}_{n}, 2$

$\mathbb{N}_{\vee}, 50,60,72,107,124-125,135$,

150

$\mathbb{N}_{\wedge}, 51,60,108,124,135,150$ 


$$
\begin{aligned}
& \nu_{A}(a), 9 \\
& \omega, \omega_{1}, 2 \\
& \mathcal{P}(S), 2 \\
& \mathcal{P}_{f}(S), 2,51 \\
& \mathbf{P}_{n}(G), 52 \\
& \mathbf{P}_{n}, 147 \\
& \mathbb{P}, 85 \\
& \pi_{A}, 4,9 \\
& \pi_{X}, 32,91,109,111 \\
& \Psi_{S}, 48,102,104 \\
& \Psi_{\beta \mathbb{Z}}, 101,181 \\
& Q_{A}, 3,8 \\
& q_{G}, 10 \\
& q_{X, G}, 115 \\
& q_{X, S}, q_{S}, 109 \\
& \mathbf{Q}_{n}(G), 52 \\
& \mathbf{Q}_{n}, 147 \\
& \mathbb{Q}, 2
\end{aligned}
$$

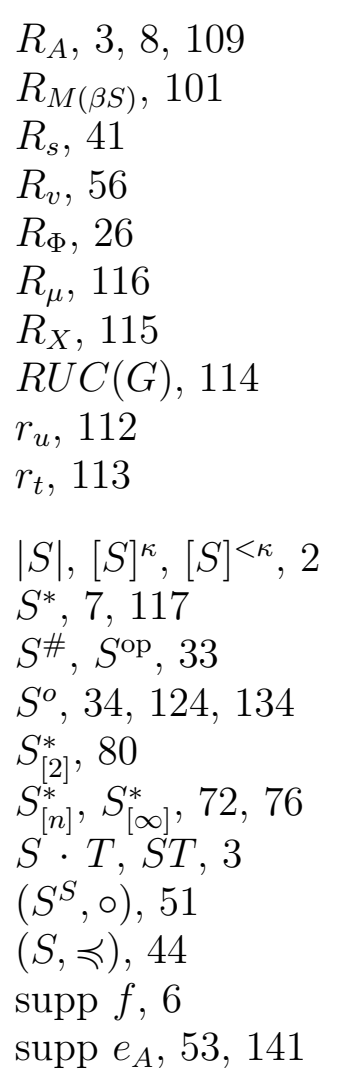

supp $\mu, 6,124$

$\mathbb{S}_{2}, 8,52,1735$

$\sigma(a), 3$

$\sigma(s), 58$

$\sigma\left(E^{\prime}, E\right), 5$

$\mathbb{T}, 2$

$T^{\star}(u), 75$

$T_{[n]}^{*}, 78,95$

$[t], 44$

$U C(G), 114$

$U V, U_{[n]}, U_{[\infty]}, 35$

$U^{-1}, U V^{-1}, V^{-1} U, 42$

$U^{*}, 67$

$U_{S}, 68$

$U_{\kappa}(S), 68,82-83,97-98$

$v(P), 25$

$W A P(A), 31$

$W A P(G), 114$

$W A P(S), 110-112,152,157,182$

$w G, 115$

$w S, 110$

$(x)_{i j}, 39$

$X^{\prime}, 32$

$\bar{X}^{\sigma}, 5$

$\mathbb{Z}, \mathbb{Z}^{+}, \mathbb{Z}_{n}^{+}, 2$

$Z(P), 61$

$Z\left(P_{G}\right), 41$

$\mathfrak{Z}(A), 2$

$\mathfrak{Z}_{t}^{(\ell)}\left(A^{\prime \prime}\right), \mathfrak{Z}_{t}^{(r)}\left(A^{\prime \prime}\right), 29,181$

$\mathfrak{Z}_{t}^{(\ell)}(\beta S), \quad \mathfrak{Z}_{t}^{(r)}(\beta S), \quad 75, \quad 107-108$,

181

$\mathfrak{Z}_{t}\left(X^{\prime}\right), 32,117$

$\mathfrak{Z}_{t}\left(L U C(G)^{\prime}\right), 118$

$\mathfrak{Z}_{t}\left(\Phi_{L U C(G)}\right), 118$

$\mathfrak{Z}_{t}\left(\Phi_{X}\right), 118$

$\square, \diamond, 26,89$

$\langle\cdot\rangle, 33$

$\leq, \leq_{L}, \leq_{R}, 35$ 